\title{
BIOMETRIC MEASUREMENTS IN THE CRYSTALLINE LENS: APPLICATIONS IN CATARACT SURGERY
}

\author{
Mengchan Sun
}

2017

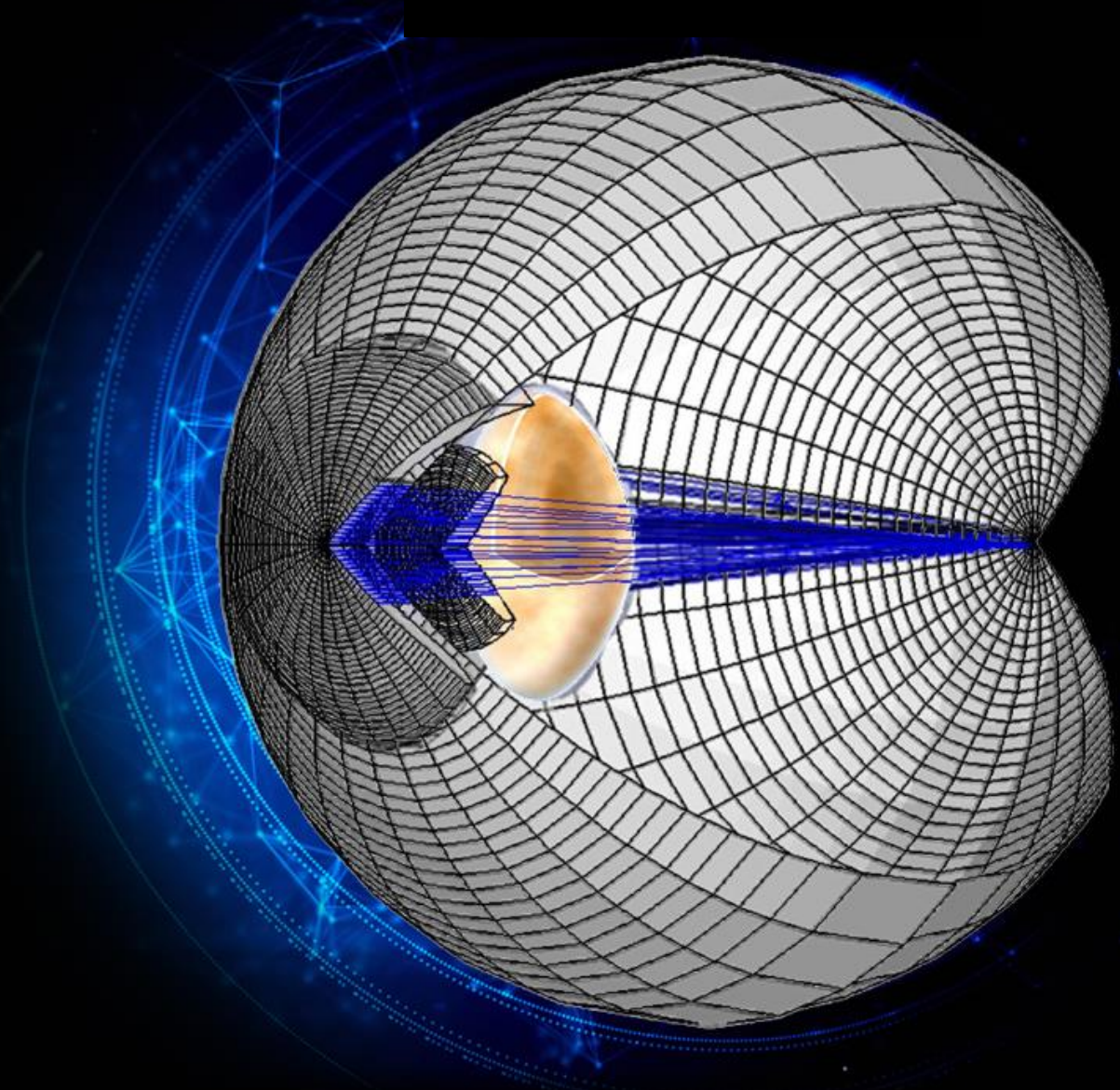

SUPERVISOR : SUSANA MARCOS INSTITUTO DE ÓPTICA. CSIC 


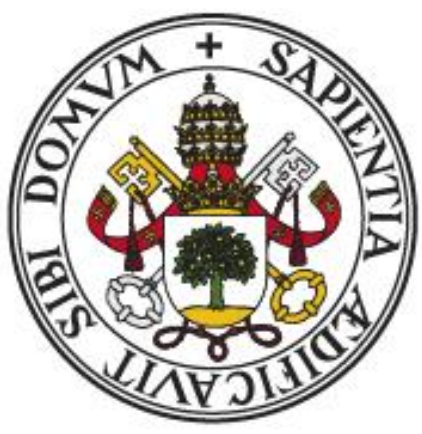

\section{Universidad deValladolid}

INSTITUTO DE OFTALMOBIOLOGÍA APLICADA

Programa de Doctorado en Ciencias de la Visión

TESIS DOCTORAL:

\section{BIOMETRIC MEASUREMENTS IN THE CRYSTALLINE LENS: APPLICATIONS IN CATARACT SURGERY}

Presentada por Mengchan Sun

para optar al grado de doctor por la Universidad de Valladolid

Dirigida por:

SUSANA MARCOS CELESTINO 


\section{Universidad deValladolid}

\section{AUTORIZACIÓN DEL DIRECTOR/A DE TESIS}

(Art. 7.2 de la Normativa para la presentación y defensa de la Tesis Doctoral en la UVa)

\section{DD ${ }^{a}$ Susana Marcos Celestino, con D.N.I. 07954600G}

Profesora de Investigación del.Instituto de Optica, Consejo Superior de Investigaciones Cientificas (IO-CSIC) Dirección a efecto de notificaciones C/Serrano n 121 Madrid (Spain) Tel: +34 915616800 (Ext: 942313)

e-mail susana@io.cfmac.csic.es como Directora de la Tesis Doctoral titulada “.Biometric measurements in the crystalline lens: applications in cataract surgery" realizada por $\mathrm{D}^{\mathrm{a}}$ Mengchan Sun alumna del Programa de Doctorado.

\section{CIENCIAS DE LA VISIÓN}

autoriza su presentación, considerando que la tesis reune trabajo original combinando desarrollo de metodologías de imagen y diseño óptico, con estudios experimentales sobre ojos ex vivo, y pacientes, interesantes aplicaciones en cirugía de cataratas, avalada por varias publicaciones en revistas de impacto en el campo de la óptica

Madrid, 09 de Enero de 2017

El/La Director/a de la Tesis,

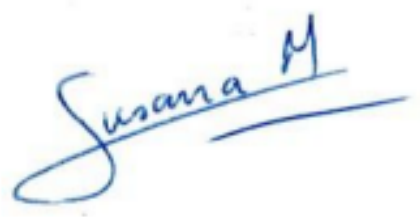

Fdo.: Susana Marcos Celestino

SR/SRA. PRESIDENTE/A DE LA COMISIÓN DE DOCTORADO 
The failuses and severses which await men - and one after another sadden the brow of youth - add a dignity to the prospect of human life, which no Arcadian success would do.

- Hensy David Tharean

尽管失败和挫折等待着人们, 一次次地夺走青春的容颜, 但却给人生的前景增添了 一份尊严, 这是任何顺利的成功都不能做到的。

献给我的父母们（孙国平, 张菊萍，郑俭，沈璐琳）和我的爱人（郑哲）

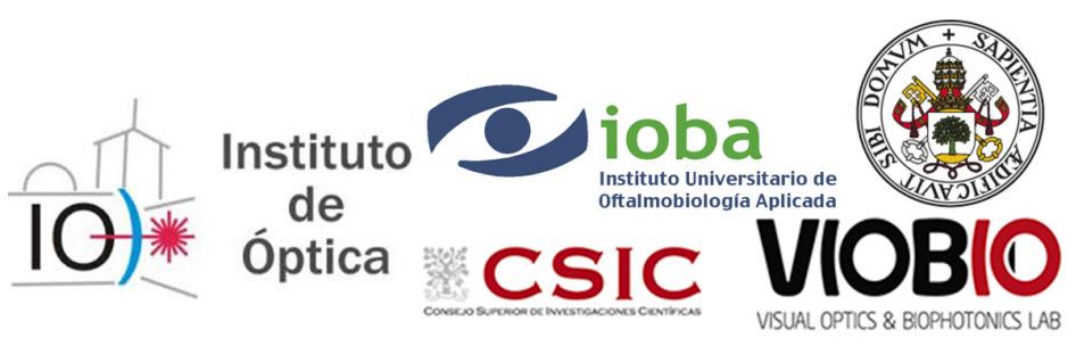

Instituto de Óptica "Daza de Valdés" (IO-CSIC) 2017

Cover designed by Mrs. Songli Zhang 


\section{$\underline{\text { TABLE OF CONTENTS }}$}

\section{BIOMETRIC MEASUREMENTS IN THE CRYSTALLINE LENS: APPLICATIONS IN CATARACT SURGERY}

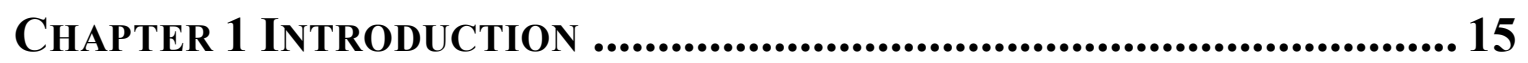

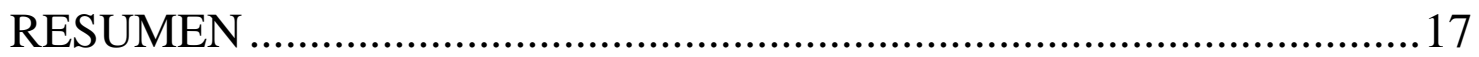

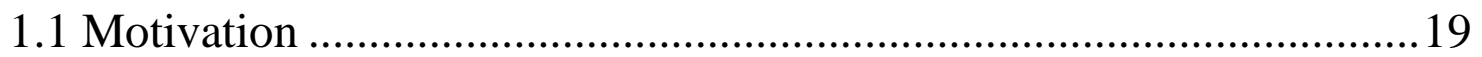

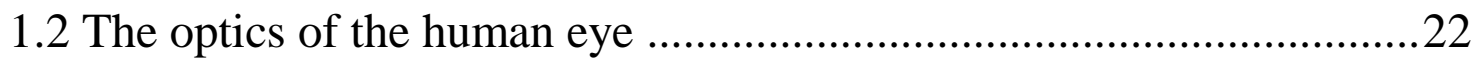

1.2.1 General structure and optical properties of normal young eyes .....................22

1.2.2 Cornea: surface shape .................................................................................2

1.2.3 Crystalline lens: structure, refractive index, surface shape and lens volume..26

1.2.4 Iris, Pupil and Ocular biomechanics................................................................ 30

1.2.5 Pupillary axis, Visual axis and Fovea........................................................... 31

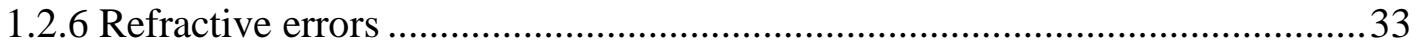

1.2.7 Optical aberrations .................................................................................... 34

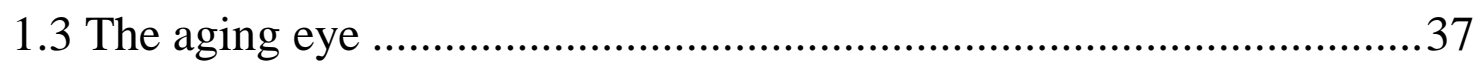

1.3.1 Presbyopia: accommodation loss of the crystalline lens ....................................37

1.3.2 Cataract: transparency loss of the crystalline lens and clinical solutions.........38

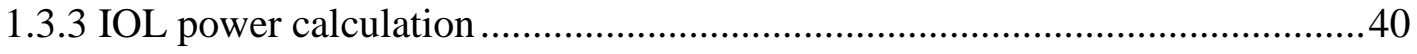

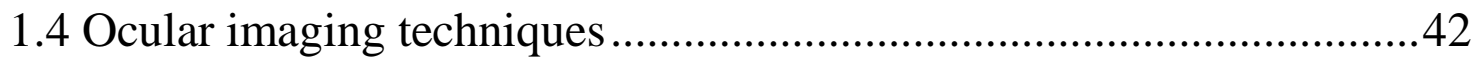

1.4.1 Scheimpflug, ultrasonography, MRI, and Purkinje imaging system................42

1.4.2 Optical Coherence Tomography (OCT) ....................................................4

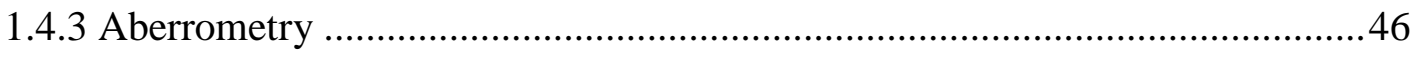

1.5 Customized eye modeling: linking geometry and aberrations .............49

1.5.1 Phakic eye: Compensation between corneal and internal aberrations..............49

1.5.2 Pseudophakic eye: contributing factors to optical degradation in eyes implanted with IOLs ..............................................................................................

1.6 Goals of this thesis and hypothesis ...................................................53

1.6.1 Goals of this Thesis ..................................................................................

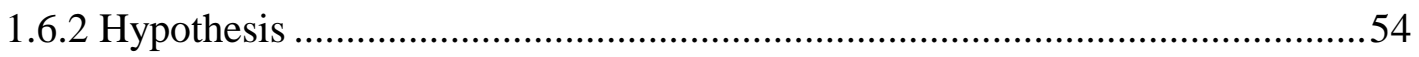

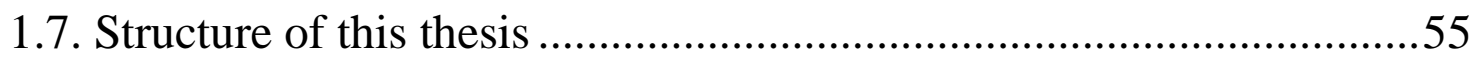


Chapter 2 EXPERIMENTAL Methods, COMPUTATIONAL TECHNiQues AND OTHER TYPES OF VALIDATION ................................................ 57

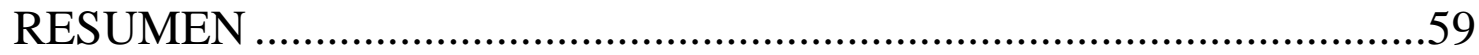

$2.13 \mathrm{D}-$ Anterior segment spectral domain OCT ...................................61

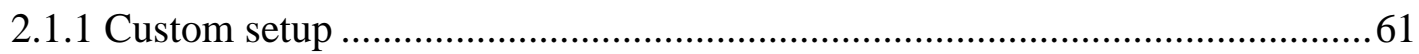

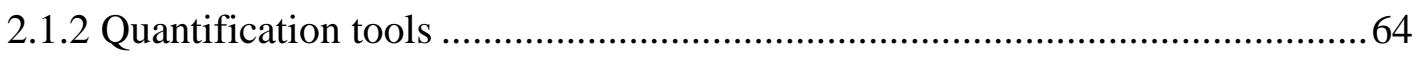

2.1.3 Purkinje-like OCT en-face reflection image to measure lens tilt and decentration

2.2 Total aberration measurement: Laser Ray Tracing (LRT) ...................73

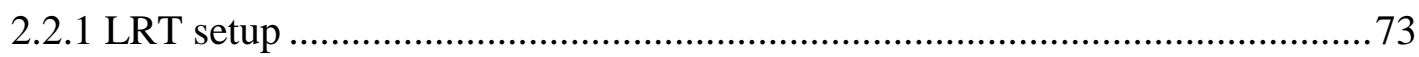

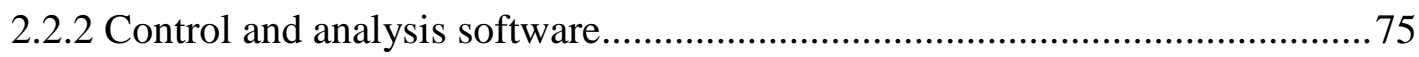

2.3 Other techniques for validation ............................................. 76

2.3.1 Pentacam: Scheimpflug camera for the validation of corneal topography......76

2.3.2 Sensofar PLu2300: Non-contact profilometry for the validation of IOL

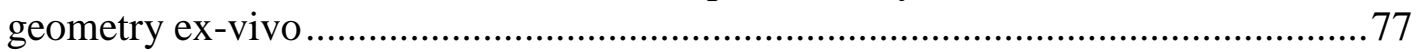

2.3.3 Water-cell physical model eye for the validation of eye rotation, IOL tilt and

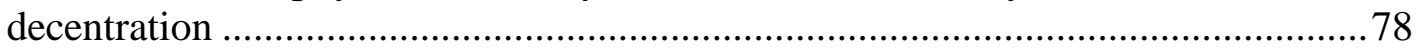

2.3.4 Custom computer model eyes ................................................................ 80

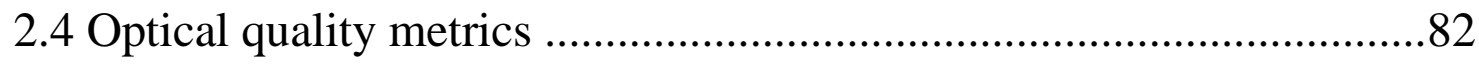

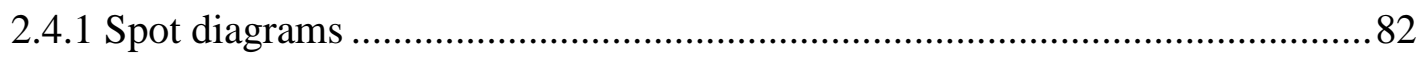

2.4.2 Zernike coefficients and RMS wavefront error ........................................... 83

2.4.3 Modulation transfer function, Strehl and visual Strehl................................ 84

$2.5 \mathrm{Ex}$ vivo and in vivo measurements............................................. 86

2.5.1 Lens specimens, preparation and experimental protocol................................86

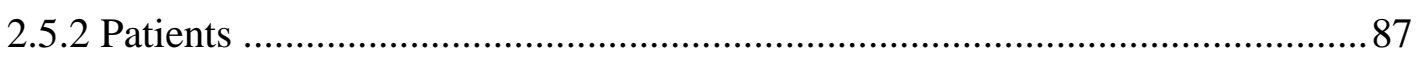

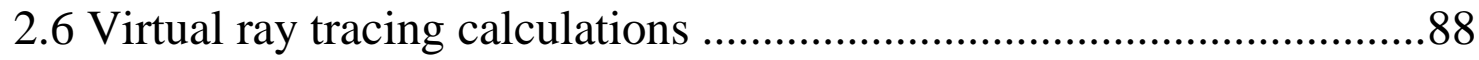

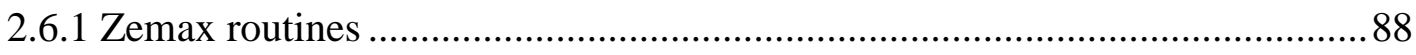

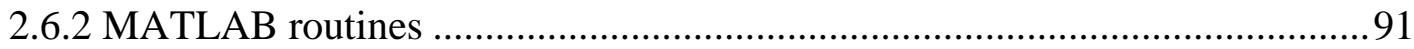

CHAPTER 3 INTRAOCULAR LENS ALIGNMENT FROM AN EN-FACE OCT IMAGE PURKINJE-LIKE METHOD ............................................................ 97

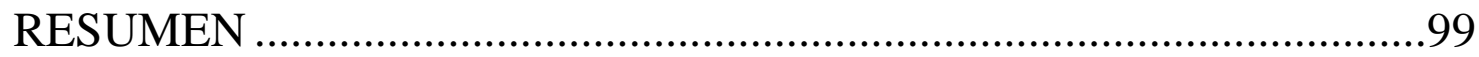

3.1 Introduction ...................................................................... 101 


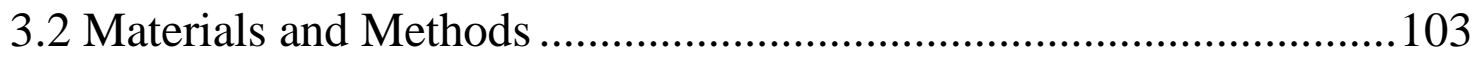

3.2.1. Laboratory-Based SD-OCT System ............................................................. 103

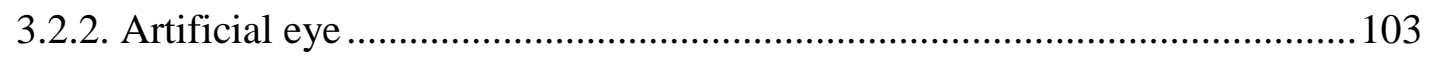

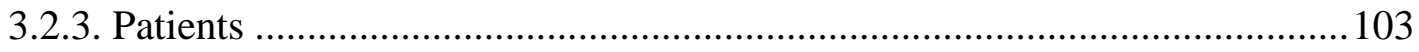

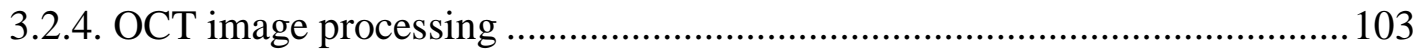

3.2.5 Purkinje-like OCT method .............................................................................. 104

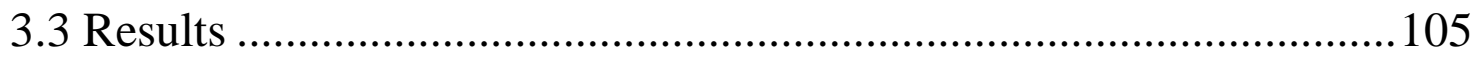

3.3.1. Validation on a water-cell physical eye model............................................ 105

3.3.2. IOL tilt and decentration in patients ............................................................ 105

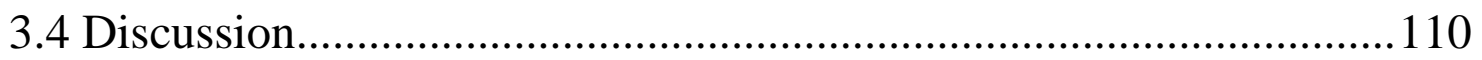

CHAPTER 4 OCT 3D SURFACE TOPOGRAPHY OF ISOLATED HUMAN CRYSTALLINE LENSES ............................................................................. 113

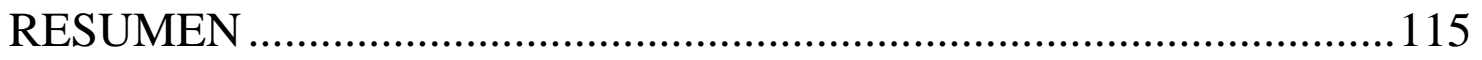

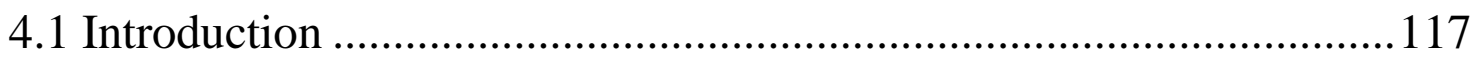

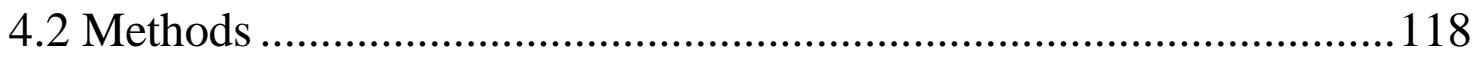

4.2.1 Lens specimens and preparation................................................................ 118

4.2.2 Experimental sOCT setup and experimental protocols ...................................118

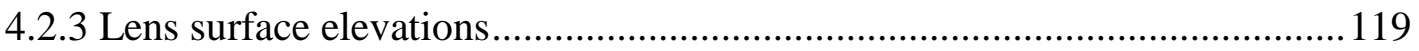

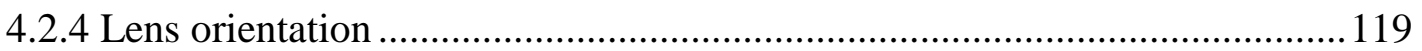

4.2.5 Power vector analysis ................................................................................. 120

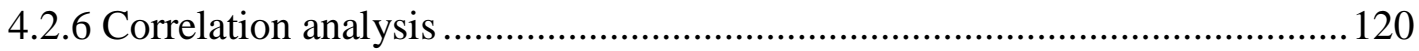

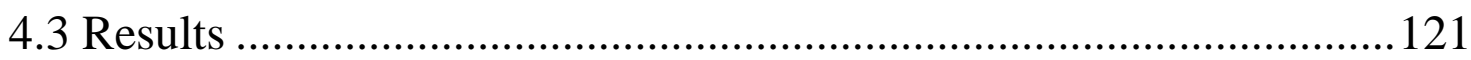

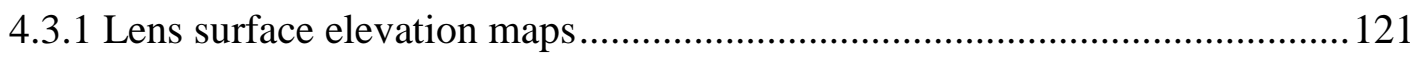

4.3.2 Lens radii of curvature and asphericity: anterior/posterior lens relationships and

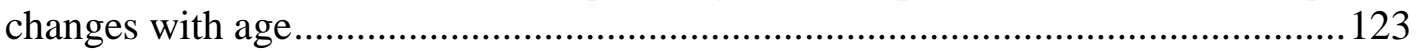

4.3.3 Changes of lens surface astigmatism and relative astigmatic angle with age124

4.3.4 Lens elevation high order Zernike terms: anterior and posterior lens

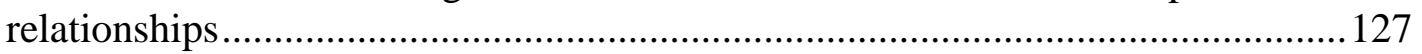

4.3.5 Lens elevation high order Zernike terms: changes with age ......................... 128

4.3.6 Relative contribution of different Zernike terms to the lens surface elevations

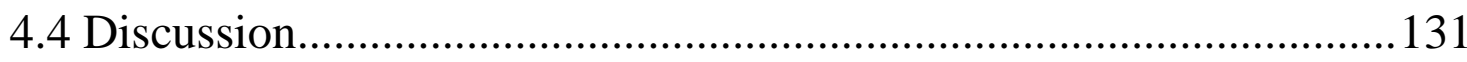


CHAPTER 5 FULL 3D OCT-BASED PSEUDOPHAKIC CUSTOM COMPUTER EYE MODEL........................................................................................ 133

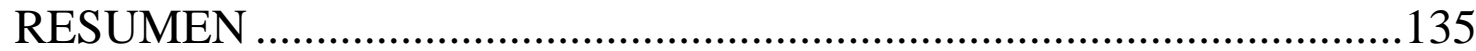

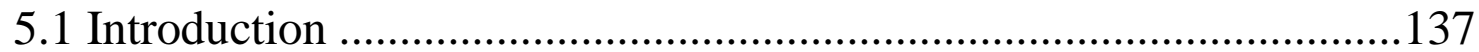

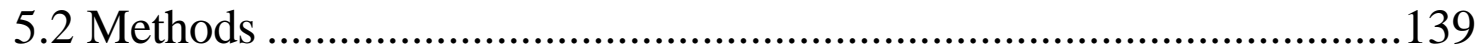

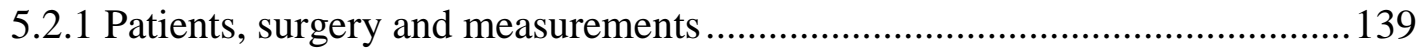

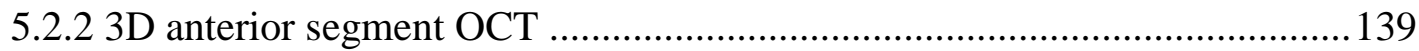

5.2.3 Ex vivo IOL geometry: non-contact profilometry .................................... 141

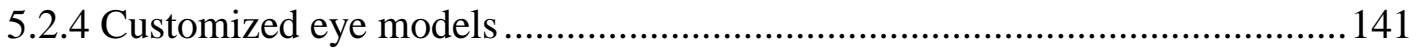

5.2.5 Total aberration measurements: LRT ….................................................... 141

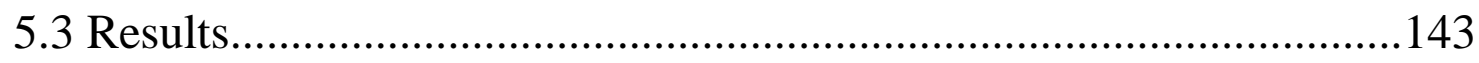

5.3.1 OCT-based anterior segment geometry in pseudophakic eyes...................... 143

5.3.2 Comparing OCT-based IOL geometry in vivo with profilometry ex vivo ... 145

5.3.3 Ocular aberrations: LRT vs. Computer Ray Tracing on custom model eye .145

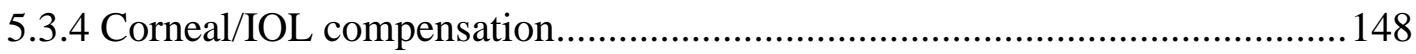

5.3.5 Effect of IOL tilt and decentration on optical quality .................................149

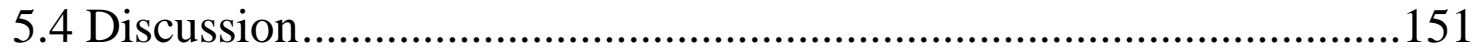

Chapter 6 OCT-BASED RAY TRACING ON PSEUdOPHAKIC EYES TO IDENTIFY OPTIMAL IOL CENTRATION ............................................ 155

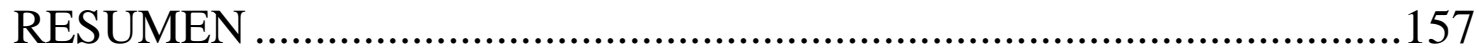

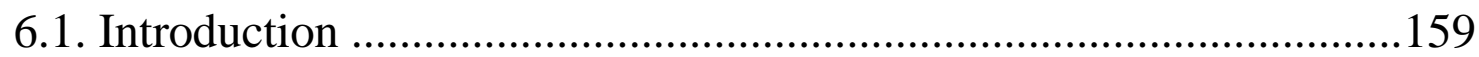

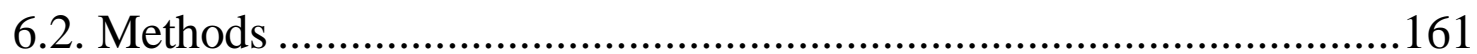

6.2.1. Patients, surgery and measurements .......................................................... 161

6.2.2. Laboratory setup: OCT, LRT and Experimental protocols .........................161

6.2.3. Purkinje-Like OCT Method.................................................................... 161

6.2.4. Full OCT-based customized eye models ...................................................162

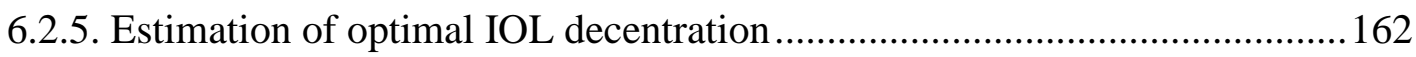

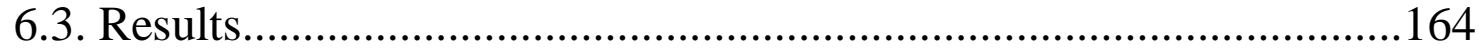

6.3.1 Measured eye rotation, IOL tilt and decentration .......................................164

6.3.2 Change of optical quality with IOL decentration using computer eye models

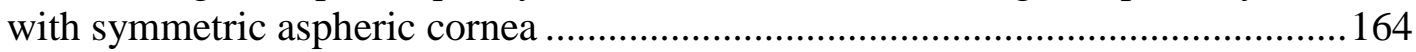

6.3.3 Change of optical quality with IOL decentration using computer eye models with real corneas 
6.3.4. Maximum Visual Strehl and Optimal IOL decentration

6.3.5. Interactions of eye rotation, IOL tilt and IOL decentration and corneal irregularities on astigmatism, trefoil and coma ................................................. 169

6.3.6. Residual defocus and residual astigmatism ............................................. 171

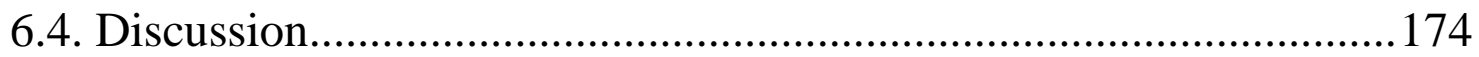

Chapter 7 Conclusions ................................................................. 175

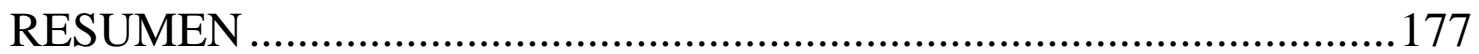

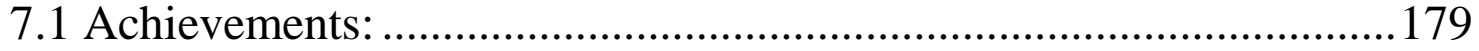

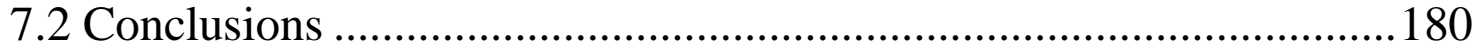

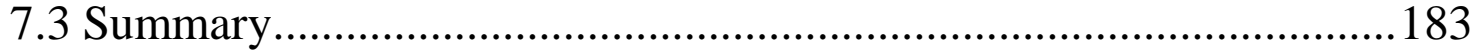

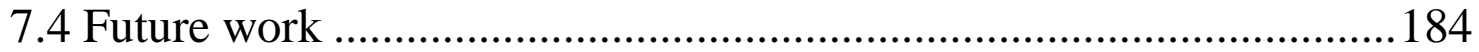

Chapter 8 Publications and Activities during the PhD Period

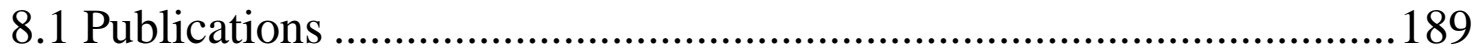

8.2 Talks and posters presented in the conferences ............................ 191

8.3 Visits and Stays in Research Institutions, Hospital and Companies.. 192

FIGURE LIST .................................................................................. 193

TABLE LIST ....................................................................... 201

BIBLIOGRAPHY ....................................................................................... 194

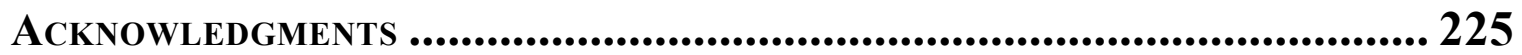




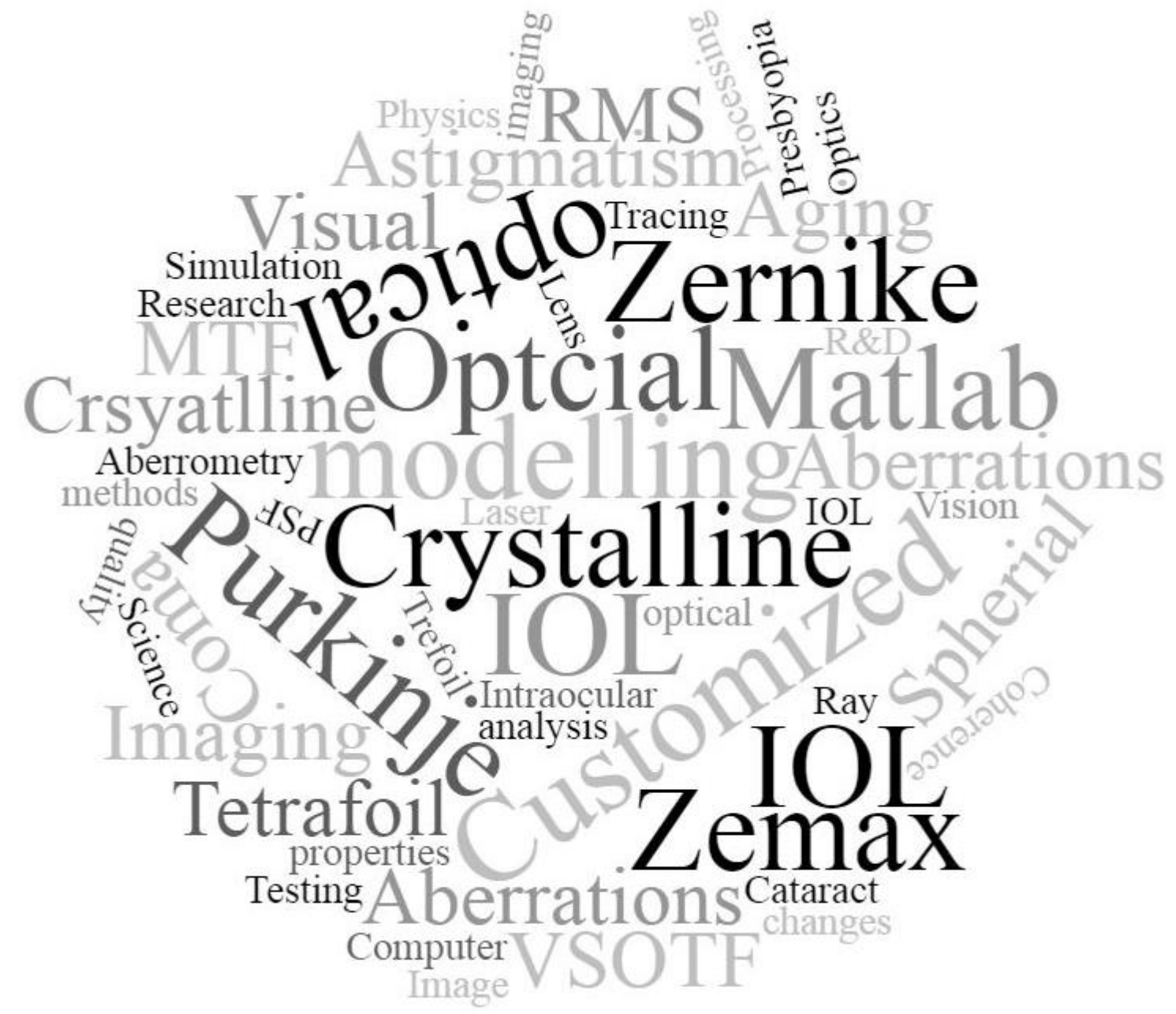




\section{LIST OF ABBREVIATIONS}

\section{A}

A = Amplitude

$\mathrm{ACD}=$ Anterior Chamber Depth

A-scan US= A-scan

ultrasonography

A-G=Purkinje weighting coefficients

$\mathrm{AL}=$ Axial Length

$\mathrm{A}-\mathrm{IOL}=$ Accommodative- $\mathrm{IOL}$

\section{B}

B-scan US= B-scan ultrasonography

BS = Beam Splitter

BST $=$ Banc de Sang i Teixits

C

$\mathrm{CSF}=$ Contrast Sensitivity Function $\mathrm{CSIC}=$ Consejo Superior de

Investigaciones Científicas

$\mathrm{CCD}=$ Charge Couple Device

$\mathrm{C}=$ Curvature $(=1 / \mathrm{R})$

\section{D}

DoF $=$ Depth-of-focus

$\mathrm{DIA}=$ the diameter of the lens at the equatorial plane

$\mathrm{D}=$ Diopters

$\mathrm{DDE}=$ Dynamic Data exchange

DoF $=$ Depth-of-Focus

DLP $=$ Digital-Light-Processing

E

$E L P=$ Estimated Lens Position

$\mathrm{ER}=$ Eye Rotation

$\mathrm{EPP}=$ Equatorial plane position

equatorial plane

F

FJD= Fundación Jiménez Díaz Hospital

FFT $=$ Fast Fourier Transform

FWHM = full-width-half-maximum

FD-OCT $=$ Fourier-Domain OCT

$\mathrm{FC}=$ Fiber Coupler
G

$\mathrm{GEO}=$ geometric spot

GRIN=Gradient refractive index

H

$\mathrm{H}=$ Horizontal

$\mathrm{H}-\mathrm{S}=$ Hartmann-Shack

$\mathrm{HOA}=$ High order aberration

I

$\mathrm{I}=$ Intensity

$\mathrm{IOL}=$ intraocular lens

$\mathrm{IR}=$ Infrared

IT $=$ IOL Tilt

ID $=$ IOL Decentration

ILT $=$ intraocular lens thickness

ILP= intraocular lens position

ICRS = Intracorneal ring segment

$\mathbf{K}$

$\mathrm{K}=$ Conic constant

$\mathbf{L}$

$\mathrm{L}=$ Lens

LRT $=$ Laser Ray Tracing

LOS= Line of sight

LCA = Longitudinal Chromatic

Aberration

LT $=$ Lens thickness

LED $=$ Light Emitting Diode

M

$\mathrm{M}=$ Mirror

MTF $=$ Modulation Transfer Fuction

MRI = Magnetic Resonance Imaging

$\mathrm{M}-\mathrm{IOL}=$ multifocal $\mathrm{IOL}$

$\mathbf{N}$

NDF $=$ Neutral Density Filter

$\mathrm{N}=$ Nasal

$\mathrm{NA}=$ Numerical Aperture 


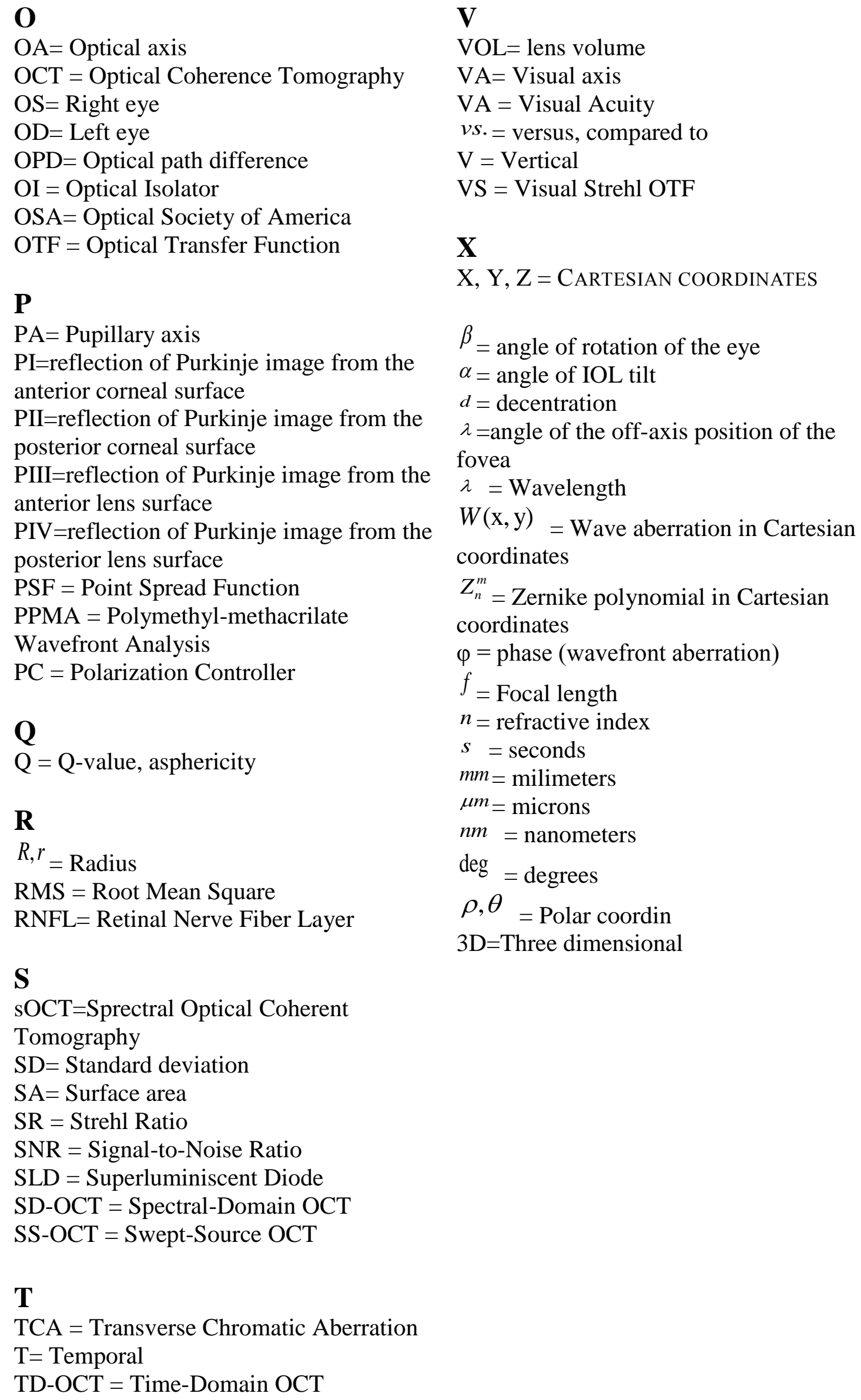




\section{Chapter 1}

INTRODUCTION 


\section{RESUMEN}

En la introducción de esta tesis, se lleva a cabo una revisión del conocimiento sobre la óptica de la lente humana, el envejecimiento del ojo, técnicas de imagen ocular, modelado de ojos personalizados. Las cataratas son el resultado de la opacificación del cristalino del ojo con el envejecimiento, que impiden la visión clara, y son una de las principales causas de baja visión en los países desarrollados y en desarrollo. La cirugía de cataratas involucra el reemplazo del cristalino por una lente intraocula (IOL) artificial. Si bien las tecnologías actuales, y en particular la selección de la la lente intraocularm, son seguras y eficaces para la mayoría de los pacientes, existen amplias posibilidades de mejora. En particular, los métodos actuales de cálculo de potencia de la IOL se basan en fórmulas regresivas, no se adaptan a pacientes con geometrías corneales especiales, y no se adaptan bien a lentes con geometría cada vez más sofisticada. Además de la pérdida de la transparencia del cristalino, antes en la vida ocurre da una périda de la capacidad acomodativa como parte del proceso de envejecimiento natural. El capítulo resume el estado del arte sobre el conocimiento del cristalino, la acomodación y envejecimiento, así como de los distintos diseños de lentes intraoculares en el mercado. Las IOLs actuales compensan los errores de refracción e incluso la compensación de la aberración esférica corneal y, cada vez más, apuntan a imitar la función de la lente en un ojo joven, incluyendo la optimización óptica e incluso el alojamiento.

La medición precisa de la geometría del cristalino es crucial en la comprensión de sus propiedades ópticas y de los cambios físicos con la acomodación y la presbicia. En los últimos 20 años se han desarrollado múltiples tecnologías para la medición de la anatomía y aberraciones oculares de los componentes ópticos del ojo, y se han adquirido importantes conocimientos sobre su contribución a la degradación de la calidad de imagen. Las técnicas avanzadas de imagen ocular implementadas en el Laboratorio de Óptica Visual y Biophotonics incluyen sistemas de rayos láser para la medición de aberraciones oculares; Un sistema de trazado de rayos láser y algoritmos de reconstrucción de la distribución del índice de gradiente en la lente cristalina; Un sensor de frente de onda Hartmann-Shack para medir aberraciones oculares; Un sistema de imagen de Purkinje para medir, in vivo, los radios de curvatura de las superficies de las lentes anterior y posterior, y la inclinación y descentración de las lentes cristalinas e intraoculares; Un sistema de imagen de segmento anterior de Scheimpflug, con algoritmos de corrección de distorsión óptica y geométrica y en particular, sistemas de tomografía de coherencia óptica de segmento anterior (OCT), proporcionando información ocular 3D cuantitativa desde la córnea anterior a la lente cristalina posterior / IOL.

Un objetivo principal de la cirugía moderna de la catarata / presbicia es lograr el resultado refractivo postoperatorio deseado y las mejoras en la predictibilidad del resultado refractivo se han debido a refinamientos en la técnica quirúrgica, particularmente pequeñas incisiones y una posición postoperatoria de IOL más predecible. Esta tesis pretende mejorar las actuales metodologías que relacionan propiedades geométricas y ópticas del ojo a través del modelo de ojo computacional personalizado, cuantificar las alineaciones de la IOL después de la cirugía, y predecir con exactitud las aberraciones oculares mediante el modelado virtual del trazado de rayos, Estos datos permitirán seleccionar la lente óptima para un paciente, así como el centrado óptico. Esta tesis hace uso de una técnica OCT cuantitativa de desarrollo propio que permite la construcción de modelos de ojo específicos para cada paciente con este propósito. 


\subsection{Motivation}

The human eye contains enough complexity to warrant specialized attention and care beyond the duties of a general practitioner. According to the latest WHO assessment, 285 million patients in the world have a visual disability (including blindness and low vision), and cataracts are responsible for $51 \%$ of world blindness, which represents about 20 million people (2010). Cataracts are the clouding of the lens of the eye that prevents clear vision, a process related to aging, and are also a leading cause of low vision in both developed and developing countries. Although cataracts can be surgically removed, in many countries barriers exist that prevent patients access to this surgery. In spite of the progress made in surgical techniques in many countries during the last ten years, cataracts remain the leading cause of visual impairment outside of developed countries $(47.9 \%)$, while the visual demands of patients in developed countries require a good optical quality for all distances. Time tested, safe and effective technologies that could restore near normal vision to a large majority of those affected are available. However, precise desirable levels of post-operative visual outcomes may be difficult to define, and reliance simply on the numbers of cataract operations performed would result in a state of undesired complacency.

The crystalline lens of the eye is an exquisite optical element, with aspheric shapes, a gradient index structure and capability of reshaping to focus near and far objects at a young age. With aging, the fine optical tuning between corneal and crystalline lens aberrations is lost. Crystalline lenses experience a decrease in the amplitude of accommodation or lose accommodative ability as part of the natural aging process. Accommodation is the process by which the vertebrate eye changes optical power to maintain a clear image as its distance varies. Further with age, the accommodation capacity is lost due to lens stiffening (presbyopia) (Pascolini, Mariotti et al. 2004, Resnikoff, Pascolini et al. 2004, Resnikoff, Pascolini et al. 2008). Converging or plus lenses for imaging near objects, which only work in spectacles or contact lenses, is one of the management techniques of presbyopia. However, changes in prescriptions are required every two to three years for presbyopia. The lens becomes opaque, and it needs to be replaced by an intraocular lens (IOL). In the middle of the $20^{\text {th }}$ century, English ophthalmologist Sir Nicholas Harold Lloyd Ridley first introduced an artificial IOL (Letocha and Pavlin 1999). A variety of different kinds of surgical procedures have been considered for restoring accommodation to the presbyopic eye such as accommodative intraocular lens (IOLs) implantation. IOLs compensate for refractive errors and, increasingly, aim at mimicking the function of the lens in a young eye, including optical tuning and even accommodation. More details about the aging eye will be described in Section 1.3.

Accurate measurement of the lens geometry is crucial in the understanding of crystalline lens optical properties and of the physical changes of the lens in accommodation and presbyopia. The lenticular accommodative biometric data most widely available refer to axial changes in the anterior segment. Anterior movement of the anterior crystalline lens surface and an increase in lens thickness has been demonstrated in several studies (Garner 1983, Zadnik, Mutti et al. 1992, Beers and Van Der Heijde 1994, Dubbelman, van der Heijde et al. 2001, Ostrin, Kasthurirangan et al. 2006, Bolz, Prinz et al. 2007). Corrected Scheimpflug images showing a posterior accommodative movement of the posterior lens surface is reported by (Dubbelman, Van der Heijde et al. 2005). In addition, the posterior 
lens surface is distorted by the refraction of the preceding optical surfaces and by the nonhomogeneous refractive index of the crystalline lens, known to have a gradient distribution (GRIN) and the presence of GRIN in the lens plays a critical role in the crystalline lens power (Pierscionek 1990). Furthermore, Kischkamp et al. reported lens tilt and decentration for unaccommodated versus accommodated states, but was unable to find statistical differences owning to the horizontal meridian, for an accommodative demand of $4 \mathrm{D}$ (Kirschkamp et al., 2004). The investigation of crystalline lenses will be valuable to the design and evaluation of accommodation-restoration solutions for presbyopia, as well as the predictability of intraocular lens implantation procedures, relies on accurate lens measurement. However, most medical image techniques (MRI, ultrasonography, videokeratoscopy, Scheimpflug etc.), are still limited by low resolution, or slower signal acquisitions.

In the last 20 years, multiple technologies have been developed for the measurement of anatomic information and correction of ocular aberrations, and important knowledge has been gained on the contribution of the different components of the eye to the degradation of image quality. Advanced ocular image techniques implemented at the Visual Optics and Biophotonics Lab include laser ray tracing systems for the measurement of ocular aberrations; a laser ray tracing system and reconstruction algorithms of the gradient index distribution in the crystalline lens; a Hartmann-Shack wavefront sensor to measure ocular aberrations; a Purkinje imaging system to measure, in vivo, the radii of curvature of the anterior and posterior lens surfaces, and tilt and decentration of the crystalline and intraocular lenses; a Scheimpflug anterior segment imaging system, with optical and geometrical distortion correction algorithms and in particularly, anterior segment optical coherence tomography systems (OCT), providing quantitative 3D ocular information from the anterior cornea to the posterior crystalline lens/IOL, could help to overcome the limits of low resolution and slow signal acquisitions. More recently quantitative customdeveloped spectral OCT (Birkenfeld, de Castro et al. 2014, Sun, Birkenfeld et al. 2014), has been used to evaluate the geometry, surface topography and gradient refractive index of the crystalline lens in vivo and ex vivo. The suitability of OCT, provided that it is corrected for fan and optical distortion (Ortiz, Siedlecki et al. 2010), to quantify the geometry of the crystalline lens has also been demonstrated in vivo in both young and old eyes(Ortiz, Perez-Merino et al. 2012, Gambra, Ortiz et al. 2013) and eyes implanted with intraocular lenses (Ortiz, Perez-Merino et al. 2013, Marcos, Ortiz et al. 2014). More currently, Perez-Merino et al. presented radii of curvature, astigmatism and high-order irregularities of the crystalline lens' anterior and posterior surfaces in accommodating eyes (Perez-Merino, Velasco-Ocana et al. 2015), and E. Martínez-Enriquez et al. studied lens volume, equatorial diameter and equatorial lens position in young eyes as a function of accommodation and in older cataract eyes as well (Martinez-Enriquez, Sun et al. 2016).

A major aim of modern cataract/presbyopia surgery is to achieve the desired postoperative refractive outcome, and improvements in the predictability of the refractive outcome have been due to refinements in surgical technique, particularly small incisions and a more predictable postoperative IOL position (Landau and Laurell 1999, Olsen 2006), as well as refinements in IOL power calculation formulas (Holladay, Prager et al. 1988, Retzlaff, Sanders et al. 1990, Hoffer 1993, Zuberbuhler and Morrell 2007, Petermeier, Gekeler et al. 2009). However, the calculation of required IOL power is currently based on regression 
formulas developed from the paraxial geometrical optics equations and/or statistical analysis of retrospective cases and quantitative imaging is essential to measure the structural properties of the ocular components and necessary to build patient-specific computer eye models. Therefore, this thesis aims at improving upon the current methodologies of linking geometrical and optical properties of the eye through customized computer modeling, to monitor the IOL alignments after surgery, to accurately predict the ocular aberrations by virtual ray tracing modeling, to identify the optimal IOL centration by an optical metric, and to predict required IOL power and best IOL through customized computer eye modeling using data from a 3D ocular biometry and geometry from a single instrument- SOCT.

Quantitative OCT will allow building patient-specific eye models for selection of the IOL design providing best optical quality. OCT-based computer eye-models have already predicted optical aberrations in pseudophakic eyes (More detail will be described in Chapter 5). The combination of high-resolution, high-speed OCT technologies with quantification tools opens up the possibility of quantifying the geometry and structure of the anterior segment of the eye, including the crystalline lens. Unlike the cornea, there are few reports on the crystalline lens shape and its change with accommodation and aging, and to our knowledge no one has investigated crystalline lens surface elevations in detail. The quantification of the anterior segment and lens is particularly important in eyes where the crystalline lens is to be replaced by an IOL, where it will be the key in the selection of the optimal IOL for a specific patient. Full 3D biometry in eyes prior to cataract surgery allows customizing the selection of the IOL to be implanted. Knowledge of the geometry and function of the young crystalline lens serves as inspiration for new accommodating intraocular lens designs (A. De La Hoz 2016). 


\subsection{The optics of the human eye}

\subsubsection{General structure and optical properties of normal young eyes}

The eye has been the subject of conflicting interpretations since antiquity. Many ancient Assyrian, Greek and Roman philosophers and physicists believed in the idea of the active eye and have tried to explain the visual process. Chinese ophthalmology was gradually formed and developed in a clinical discipline under the fight against eye disease for thousands of years. During the development of the Song and Yuan dynasties (AD 891-979), Chinese ophthalmology gradually formed a theoretical system and treatment characteristics of the discipline, which differentiated into specialized conditions. The independence of ophthalmology and specialization in science also greatly promoted the development of Chinese ophthalmology academically and technologically. In the $11^{\text {th }}$ century, Egyptian physicist Alhazen and Bukharan scientist Avicenna offered a more systematic critique of the Galenic theory of the eye, which adhered to the Aristotelian theory. In the $17^{\text {th }}$ century, German Scientist Johannes Kepler proposed a full ray tracing model of the eye and described the use of spherical lenses to correct myopic and hyperopic refractive errors. In the early $19^{\text {th }}$ century, English Scientist Thomas Young studied astigmatism, aberrations and was the first to recognize loss of accommodation with age, and western ophthalmology knowledge began to appear in Chinese medical books with the penetration of Western medicine.

The principles of image formation by the eye are the same as those for manmade optical systems. Light enters the eye through the cornea and is refracted by the cornea and lens. The general structure (Figure 1.1(a)) consists of the cornea, iris, pupil, crystalline lens, retina, macula, fovea and blood vessels. The cornea is transparent and approximately spherical with an outer radius of curvature of about $8 \mathrm{~mm}$, contributing between $65-75 \%$ of the eye's total focusing power. Behind the cornea is the anterior chamber, which is filled with a slightly saline fluid of refractive index 1.336, known as the aqueous humour (Goel, Picciani et al. 2010). At the rear of the anterior chamber is the crystalline lens. The pupil plays an important optical function through the size of its aperture. The pupil size is varied by the iris from a $2 \mathrm{~mm}$ to $8 \mathrm{~mm}$ diameter under very bright light and in darkness respectively(Winn, Whitaker et al. 1994). The aperture stop is a very important component of an optical system, affecting a wide range of optical processes. The temporal field of the human eye is about $105^{\circ}$, while the nasal field is only about $60^{\circ}$ due to both the nose and the limited extent of the temporal retina. The use of two eyes provides better perception of the external world than one eye alone, and binocular overlap is $120^{\circ}$ (in Figure 1.1 (b)). 


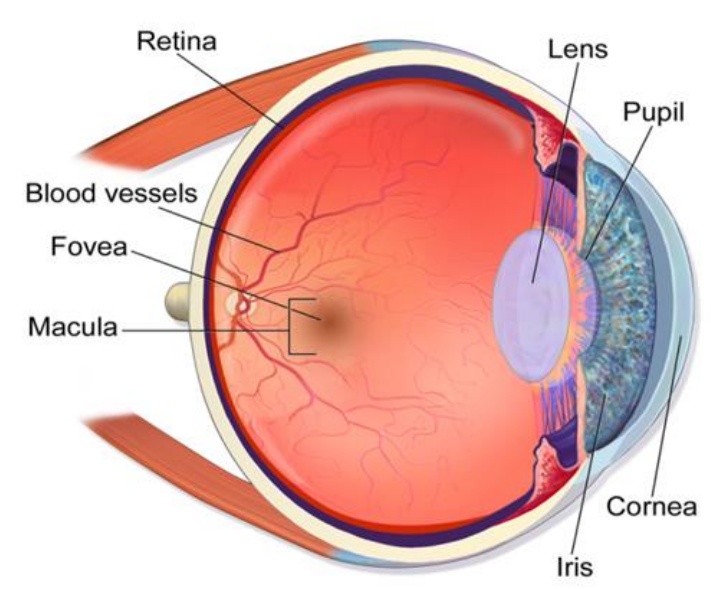

(a)

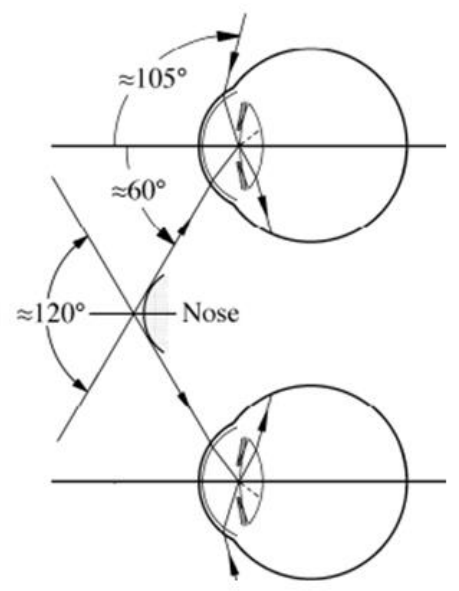

(b)

Figure 1.1 (a) The general layout of a normal young eye: cornea, iris, pupil, crystalline lens, retina, macula, fovea and blood vessels; (b) The limitation of temporal and nasal field of vision (source: Book: Optics of the Human eye).

The crystalline lens of the eye is about $3 \mathrm{~mm}$ inside the eye and is connected to the ciliary body by suspensor ligaments called zonular fibers. The lens shape can be altered to change its power when the eye needs to focus at different distances (accommodation). The posterior chamber is between the iris, the ciliary body and the lens, and contains aqueous humour. The inner layer of the eye is the retina, which is an extension of the central nervous system and is connected to the brain by the optic nerve, and the vitreous chamber between the lens and the retina. Bekerman et al. reported the normative data on eyeball diameters among 250 adults with healthy eyes. The eyeball size may vary from $21 \mathrm{~mm}$ to $27 \mathrm{~mm}$ (Bekerman, Gottlieb et al. 2014) among individuals, with average values of anterior chamber depth (3.3 $\mathrm{mm}$ ), lens thickness $(3.5 \mathrm{~mm})$, radii of curvature of lens surfaces (anterior: $11 \mathrm{~mm}$; posterior: $-6 \mathrm{~mm}$ ) depending upon gender, accommodation and age, but those are known to vary widely in the population.

\subsubsection{Cornea: surface shape}

The refracting components of the eye are the cornea and the lens. Those two elements are transparent and have appropriate curvatures and refractive indices, with refraction taking place at four surfaces-the anterior and posterior surfaces of the cornea and lens. Ideally, when the eyes fixate on an object of interest, the image is sharply focused on the fovea. The cornea is the eye's outermost layer, which is a clear, dome-shaped surface. The cornea is a highly organized group of cells, proteins and epithelium. Bowman's membrane, stroma, Descemet membrane and endothelium are the five main corneal layers in human eyes (shown in Figure 1.2). The epithelium protects the rest of the cornea and is coated with the tear film, larger molecules and toxic substances. Bowman's layer is around $10 \mu \mathrm{m}$ thick and consists mainly of randomly arranged collagen fibrils. The bulk of the cornea is formed by the stroma, which in the human adult is approximately $500 \mu \mathrm{m}$ thick centrally (approximately $90 \%$ of corneal thickness) and is composed predominantly of flattened and stacked collagenous lamellae (around 200 layers). Descemet's membrane is the basement of the endothelial cells. The endothelium is a single layer of cells, which are hexagonal and fit together like a honeycomb. 
The cornea fulfills a dual role, acting as both the primary refractive element and as a physical barrier to maintain ocular integrity. The cornea is a viscoelastic tissue that responds to the presence of external and internal forces (Meek and Newton 1999, Roberts 2000, Dupps and Wilson 2006, Elsheikh, Alhasso et al. 2008, Kling 2009, Meek and Knupp 2015). One of the most important properties of any optical system is the equivalent power. The average power of the cornea is 40 diopters (D), which is $2 / 3 \mathrm{rd}$ of the power of the whole eye. Although the anterior corneal surface is not smooth due to its cellular structure, an optically smooth surface is achieved with a very thin tear film, which covers the cornea. The posterior surface is more curved than the anterior surface, while the anterior surface has a greater power (48D) than the posterior surface (-8D) owing to the low refractive index difference between the cornea and aqueous humour. The refractive index of the cornea is generally considered as homogeneous with an accepted value of 1.376. Some authors have addressed the change of corneal refractive index with wavelength (Sivak and Mandelman 1982) or described the cornea as a gradient structure (Patel, Marshall et al. 1995, Barbero 2006).

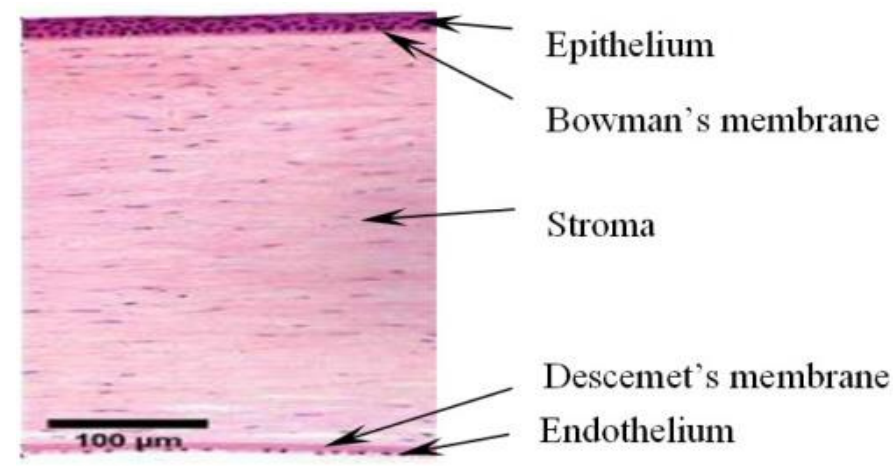

Figure 1.2 Histological section of a human cornea (source: www. missionforvisionusa. org).

Pathologies that affect the normal shape of the cornea, such as keratitis, corneal degeneration and dystrophy, congenital and abnormal corneal development, ect., degrade corneal optical quality and consequently retinal image quality. The first studies proposed by Ramsden in 1779 were performed on anterior corneal surfaces with keratometers, but the device can only be applied to a very limited and central region of the cornea. Later in the $19^{\text {th }}$ century, Donders provided the first anterior corneal data of males and females. Other authors, including Helmholtz, Javal and Schiotz, used different generations of keratometers to characterize the anterior radius of the cornea (Sorsby, Benjamin et al. 1957, P. H. Kiely 1982, Guillon, Lydon et al. 1986, Patel, Marshall et al. 1993, Lam, Jiang et al. 1997). More recently, Dubbelman et al. used a Scheimpflug camera, which had algorithms to compensate for the refractive error of the anterior surface, to measure that shape of the posterior corneal surface with respect to several meridians (Dubbelman, Weeber et al. 2002).

Dubbelman et al. reported that the average radius was $7.79 \pm 0.27$ (SD) $\mathrm{mm}$ and $6.53 \pm 0.25$ (SD) $\mathrm{mm}$ of the anterior and posterior corneal surfaces respectively. Both surfaces were found to be flatter horizontally than vertically. The cylindrical component of the posterior surface of $0.33 \mathrm{~mm}$ is twice that of the anterior surface $(0.16 \mathrm{~mm})$. Asphericity of corneal surfaces influences higher order aberrations (HOA). In contrast to the anterior corneal 
surface, the asphericity of the posterior corneal surface varied significantly between meridians.

With age, the asphericity of both the anterior and the posterior corneal surface changes significantly, which results in a slight peripheral thinning of the cornea, and the intersubject variability in these values is very high (Dubbelman, van der Heijde et al. 2005). Modern measurement of corneal curvature/power can be performed with a variety of instruments. For example, the most common instruments found in optical biometry systems include keratometers, the IOLMaster (Carl Zeiss AG, Jena, Germany) or Lenstar (Haag-Streit, USA), corneal Videokeratography and Scheimpflug based corneal topography. More recently, out laboratory has validated the use of Optical Coherence Tomography (OCT) as a quantitive anterior and posterior corneal topographer (Ortiz, Siedlecki et al. 2009, Ortiz, Siedlecki et al. 2009, Ortiz, Siedlecki et al. 2010, Ortiz, Siedlecki et al. 2011). Ray tracing through OCT-based corneal surface elevations has allowed, among others, computation of corneal aberrometry, showing high degrees of agreement with total aberrometry in an of group keratoconic eyes pre- and post- implantation of intrastromal corneal ring segments (Perez-Merino, Ortiz et al. 2014). Corneal irregularities in the elevation map and astigmatism are common. In astigmatism, the eye produces an image with multiple focal points or lines. In regular astigmatism, there are two principal meridians, with constant power and orientation across the pupillary aperture, resulting in two focal lines. The astigmatism is then further defined according to the position of these focal lines with respect to the retina. When the principal meridians are at right angles and their axes lie within 20 degrees of the horizontal and vertical, the astigmatism is subdivided into astigmatism with the rule, in which the greater refractive power is in the vertical meridian; and astigmatism against the rule, in which the greater refractive power is in the horizontal meridian. Astigmatism with the rule is more commonly found in younger patients and astigmatism against the rule in older patients (Figure 1.3). Oblique astigmatism is regular astigmatism in which the principal meridians do not lie within 20 degrees of the horizontal and vertical meridians. Corneal surface high order terms, which some authors denominate as irregular astigmatism, are also frequent (Koh, Maeda et al. 2002, Kuroda, Fujikado et al. 2002, Maeda, Fujikado et al. 2002, Hayashi and Hayashi 2006). Interestingly, the posterior corneal surface appears to compensate for part of the errors of the anterior cornea. In particular, the posterior cornea has been reported to compensate around $3.5 \%$ of the coma of the anterior surface (Dubbelman, Sicam et al. 2007). There are also numerous reports of the compensation of the corneal astigmatism by the astigmatism of the crystalline lens using corneal and refractive parameters (Kelly, Mihashi et al. 2004, Lim, Gazzard et al. 2008, Park, Oh et al. 2013, Kotecha, Russell et al. 2014). More detail on astigmatism and optical aberration will be described in Section 1.2.6. Axis misalignment of the anterior and posterior corneal astigmatism has been reported before (Thibos and Horner 2001) as well as misalignments in the astigmatic axes of the anterior and posterior crystalline lens surfaces (Kamiya, Shimizu et al. 2015). More detail will be provided in the study of OCT 3D surface topography of isolated crystalline lenses in Chapter 4. 


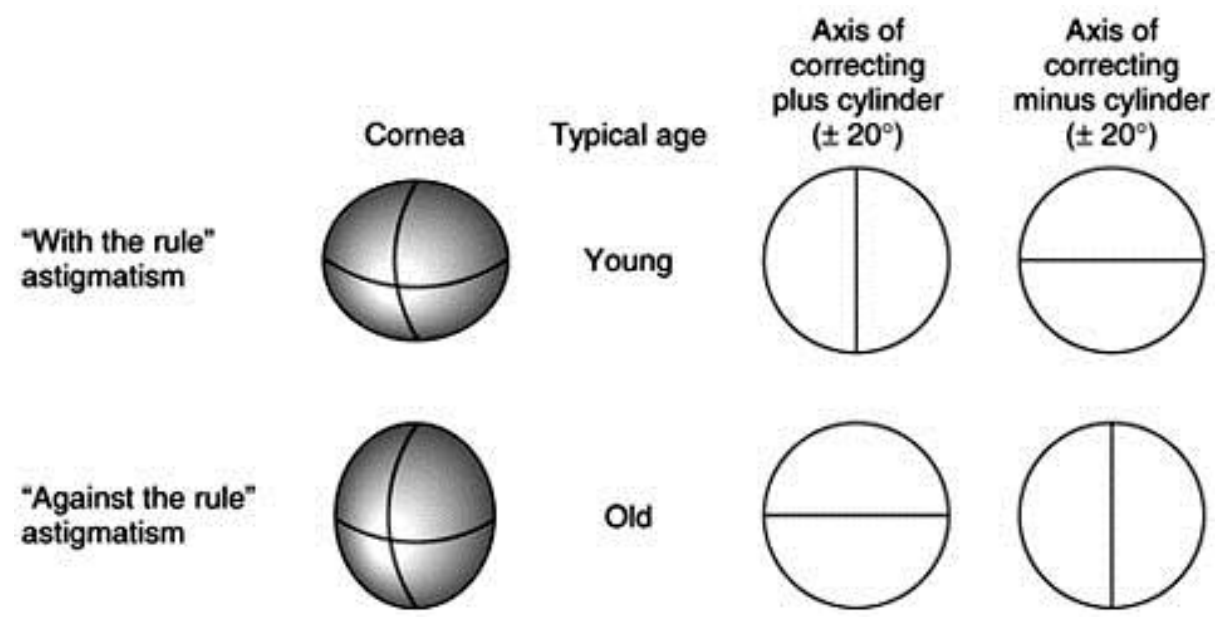

Figure 1.3 Types of astigmatism as determined by the orientation of the principal meridians and the orientation of the correcting cylinder axis. [Paul Riordan-Eva et al., 2003, source:http://www.oculist.net/others/ebook/generalophthal/server-java/arknoid/amed/ vaughan/ co_chapters/ch020/ch020_print_01.html]

\subsubsection{Crystalline lens: structure, refractive index, surface shape and lens volume}

The crystalline lens of the eye is a fascinating optical element, which, together with the cornea, transmits and refracts light to form an image of the world on the retina. The understanding of the properties of the crystalline lens of the human eye is crucial for the design and evaluation of solutions for presbyopia and for cataracts. The crystalline lens, shows an ellipsoidal, biconvex shape, and is composed of 4 layers, from the surface to the center: capsule, subcapsular epithelium, cortex and nucleus, shown as Figure 1.4. The lens capsule is a clear, membrane-like structure that is elastic, a quality that keeps it under constant tension. Subcapsular epithelium is responsible for the continuous growth of the lens throughout life with new epithelial cells forming at the equator. At the equator, away from the light path, epithelial cells undergo mitotic division and differentiate into lens fibers (Beebe 2003). The nucleus of the lens shows the highest refractive index, whereas the more peripheral fibers from the cortex present the smallest refractive index in the lens (Von Helmholtz 1909, Atchison and Smith 1995, Goncharov and Dainty 2007, de Castro, Siedlecki et al. 2011, Siedlecki, de Castro et al. 2012).

Furthermore, the distinctive concentration of different proteins produces changes in the refractive index across layers (Beebe 2003). Recent work has shown that in human (Birkenfeld, de Castro et al. 2014), as well as monkey (de Castro, Birkenfeld et al. 2013) and porcine lenses (Birkenfeld, de Castro et al. 2013), both the lens shape and the gradient refractive index (GRIN) play a role in the negative spherical aberration of the lens. De Castro, A. et al. (de Castro, Ortiz et al. 2010) reported index variation in the nucleus with values ranging from 1.434 to 1.413 and in the lens cortex with values ranging from 1.386 to 1.376 , showing a monotonic decrease in young crystalline lenses and a plateau-like profile in older crystalline lenses. Overall, the spherical aberration of the eye shifts toward more positive values with age (Glasser and Campbell 1998, McLellan, Marcos et al. 2001, Amano, Amano et al. 2004). To date, most GRIN measurements (and all optical measurements of the GRIN) have been performed ex vivo (Jones, Atchison et al. 2007, de 
Castro, Siedlecki et al. 2011, Birkenfeld, de Castro et al. 2013). The use of an equivalent index of refraction that represents the refractive property of the GRIN (i.e. which in combination with the cornea and lens curvatures) predicts the eye's spherical error is frequent (Dubbelman, Van der Heijde et al. 2005, Uhlhorn, Borja et al. 2008, Birkenfeld, de Castro et al. 2014). For a homogenous lens to have the same power, the bulk index would have to be greater than the peak index in the gradient. Little refraction takes place at the surface but instead the light curves as it passes through.

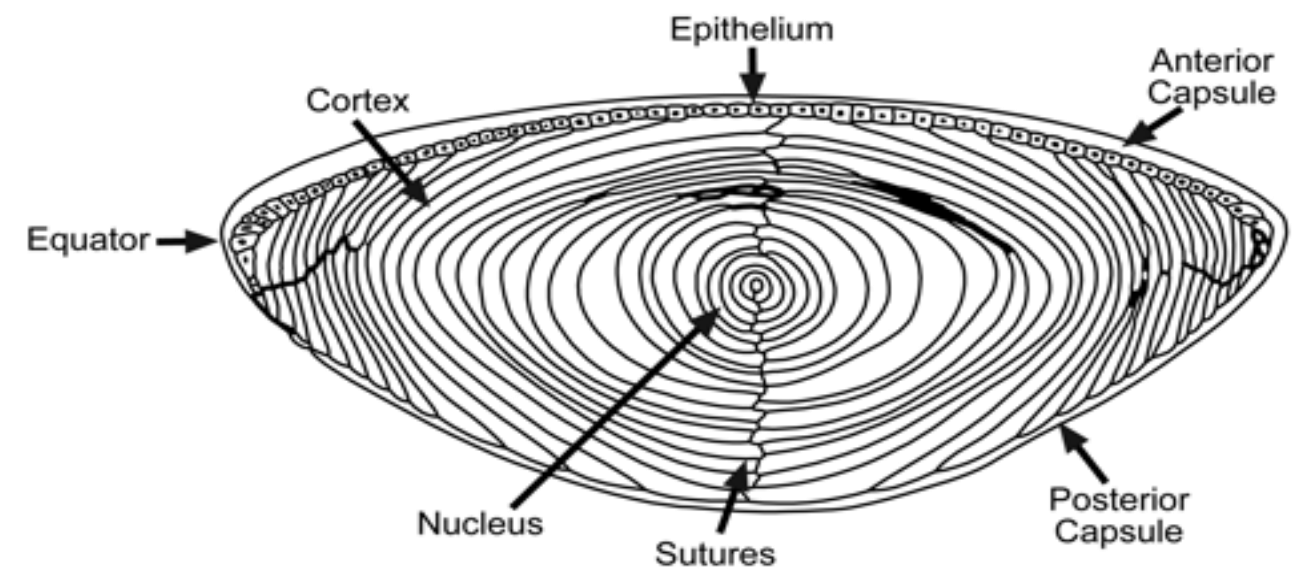

Figure 1.4 The structure of the Human Lens. Suture and Equator are anatomical terms in ophthalmology. Suture means the seams of the lens. The suture patterns become more complex as more layers of lens fibers are added to the outer portion of the lens. Equator means the edge of the largest portion of the lens (similar to the equator on a globe) (source: http://photobiology.info/Roberts.html)

In-vivo measurements of the crystalline lens radii of curvature typically come from Purkinje (Garner and Yap 1997, Rosales, Dubbelman et al. 2006, Rosales and Marcos 2006), Sheimpflug (Cook, Koretz et al. 1994, Dubbelman and Van der Heijde 2001, Dubbelman, van der Heijde et al. 2001, Koretz, Cook et al. 2001, Dubbelman, Van der Heijde et al. 2003, Dubbelman, Van der Heijde et al. 2005, Rosales, Dubbelman et al. 2006), Magnetic Resonance Imaging (MRI) (Atchison, Markwell et al. 2008, Hermans, Pouwels et al. 2009, Kasthurirangan, Markwell et al. 2011, Sheppard, Evans et al. 2011), and Optical Coherence Tomography (OCT) (Ortiz, Perez-Merino et al. 2012, Gambra, Ortiz et al. 2013, Perez-Merino, Velasco-Ocana et al. 2015). Manns et al. reported, for the first time, measurements of the lens asphericity ex vivo (Manns, Fernandez et al. 2004). Interaction effects between the lens radii of curvature and asphericity in the lens profile fittings suggest that those values should not be interpreted separately (Perez-Escudero, Dorronsoro et al. 2010). Dubbelman et al. showed that the asphericity of human lenses in vivo tends to increase with age for both anterior and posterior surfaces(Dubbelman and Van der Heijde 2001). These results are in agreement with the report by Birkenfeld et al on ex vivo lenses (Birkenfeld, de Castro et al. 2014). Due to its inaccessibility, the in vivo geometrical parameters of the crystalline lens are limited in the literature. Rosales et al., (Rosales and Marcos 2006, Rosales and Marcos 2009) using a distortion-corrected Scheimpflug camera, reported averaged values of the crystalline lens radius for the anterior surface $(11.1 \pm 1.1$ $\mathrm{mm}$ ) and for the posterior surface $(6.1 \pm 0.5 \mathrm{~mm})$. Dubbelman et al. (Dubbelman and Van der Heijde 2001) described age-related expressions for the anterior and posterior crystalline 
lens surfaces respectively $(\mathrm{R}=12.9-0.057 \mathrm{x}$ age, anterior; $\mathrm{R}=6.2-0.012 \mathrm{x}$ age, posterior), and reported an average conic constant value of -4 and -3 for the anterior and posterior lens, respectively. Average lens thickness values range between $3.06 \mathrm{~mm}$ to $4.19 \mathrm{~mm}$ at 30 years of age with a mean increase of $24 \mu \mathrm{m} /$ year (Dubbelman and Van der Heijde 2001).

Ortiz et al., (Ortiz, Perez-Merino et al. 2012) quantified the crystalline lens geometrical properties with distortion-corrected OCT, and reported the first in vivo 3D surface elevation maps of the anterior and posterior lens surfaces as shown in Figure 1.5. These authors noted that the axes of astigmatism in the anterior and posterior lens surfaces tended to be orthogonal in the three subjects of their study. Data ex vivo and in vivo differ primarily because the isolated lens appears in its maximally accommodated state, and therefore the young lens shows large steepening ex vivo. Ex vivo, the anterior and posterior lens surface radii of curvature tend to increase with age (up to or past the presbyopia onset) (Manns, Fernandez et al. 2004, Birkenfeld, de Castro et al. 2014), while in vivo, both surfaces tend to steepen with age (Dubbelman and Van der Heijde 2001).

Much of the knowledge on the optics of the crystalline lens comes from wavefront aberration measurements. Hartmann-Shack measurements of the crystalline lens in rhesus monkeys suggest the presence of high order aberrations beyond spherical aberration (Roorda and Glasser 2004). Artal et al. measured the wavefront aberration of the crystalline lens in vivo by neutralizing the contribution of the cornea with the use of goggles filled with solution (Artal, Guirao et al. 2001). Several other works estimate the internal optics obtained by subtraction of the corneal aberrations from the total aberrations (Artal, Guirao et al. 2001, Barbero, Marcos et al. 2002). These studies confirm the previously reported balance between the positive spherical aberration of the cornea and the negative spherical aberration of the crystalline lens (Sivak and Kreuzer 1983). However, this methodology does not permit identification of the relative contribution of irregular shape in the crystalline lens and ocular misalignments to the lens wavefront aberration. In particular, there is evidence that the balance between horizontal coma in the cornea and the crystalline lens arises primarily from the eccentric position of the fovea, resulting in an angular separation between the line of sight and the optical axis, known as angle lambda (Kelly, Mihashi et al. 2004, Marcos and Rosales et al. 2008). Early attempts to measure the astigmatism of the internal ocular surfaces involved the use of videokeratography, A-scan ultrasonography, and autorefractometry along with multi-meridional phakometric measurements of Purkinje images (Rosales and Marcos 2006). As noted in the literature (Barry, Dunne et al. 2001), the ophthalmo-phakometric method was prone to considerable accumulated experimental errors. However, the work reports some interesting findings, such as predominance of inverse astigmatism (where the steeper meridian is in the horizontal axis) in the posterior cornea and in the posterior lens surface, while the astigmatism of the anterior surface of the lens was direct (where the steeper meridian is in the vertical axis). 


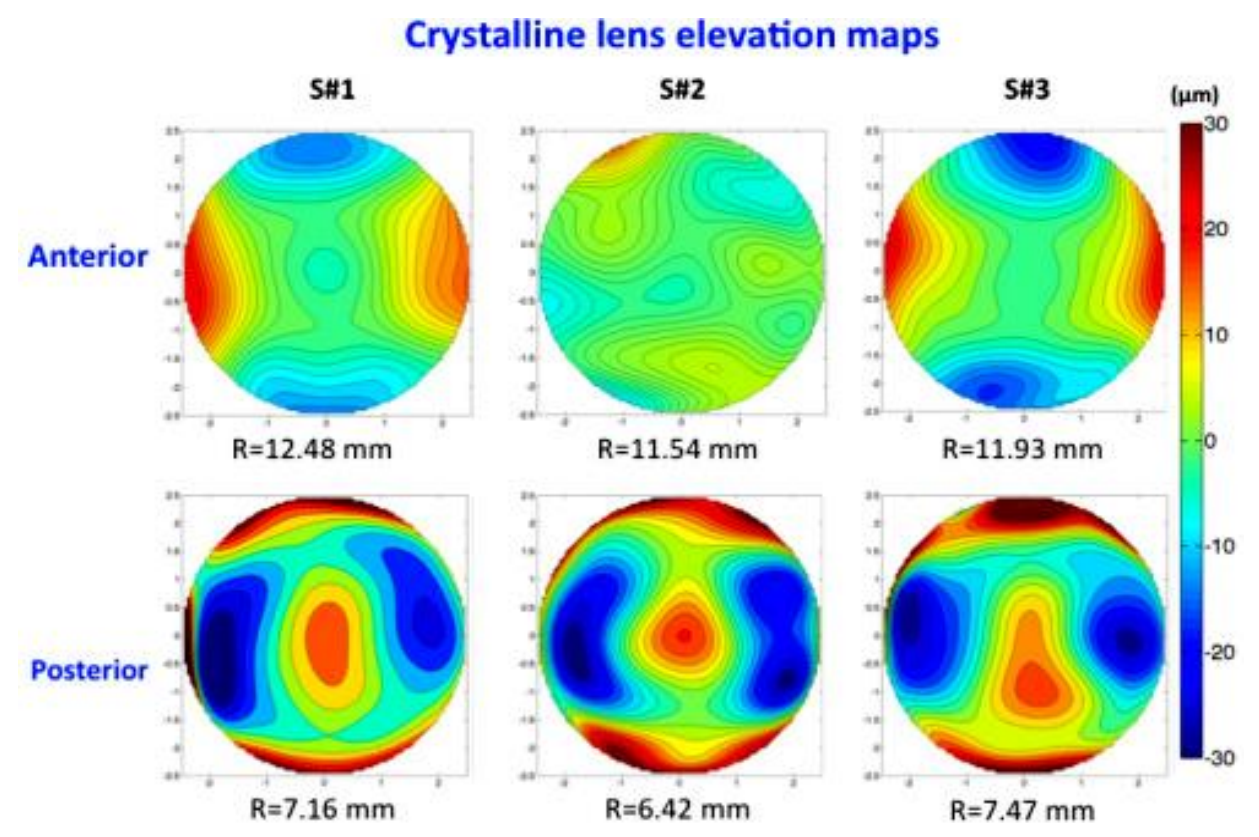

Figure 1.5 Quantitative elevation maps of the posterior lens surface for an in vitro 65-year donor lens. Left panel: Measurements with the posterior surface of the lens facing the OCT beam ("posterior up"); Middle panel: Measurement of the posterior surface of the lens viewed through the anterior surface of the lens, and no optical distortion correction (simple division by the index of refraction); Right panel: Measurement of the posterior surface of the lens viewed through the anterior surface of the lens, after application of optical distortion correction. Maps are fit relative to the best fitting sphere with Zernike polynomials. $\mathrm{R}$ is the radius of curvature of the best fitting (Ortiz, Perez-Merino et al. 2012).

Besides lens phakometry, asphericity, and astigmatism, lens GRIN and lens aberrations, a scarce numbers of studies have reported mostly the ex vivo shape of the entire lens and the associated lens parameters, e.g., equator plane position, volume or diameter of lens surface. In vivo reports based on MRI (Atchison, Markwell et al. 2008, Hermans, Pouwels et al. 2009, Kasthurirangan, Markwell et al. 2011, Sheppard, Evans et al. 2011) of crystalline lenses determined the lens volume (VOL) and surface area (SA) of the capsular bag and investigated whether there is a change in these quantities with accommodation, although they were limited by the relative low resolution of the technique. The preservation of lens volume implies that the internal human lens material can be assumed to be incompressible and is undergoing elastic deformation. Furthermore, the change in surface area indicates that the capsular bag also undergoes elastic deformation (Hermans, Pouwels et al. 2009).

The change in VOL could indicate that decompression of the internal lens material is responsible for the accommodative changes. In addition, optical imaging in the eye only allows for the retrieval of information visible through the pupil, preventing direct calculation of some important parameters such as the equatorial plane position (EPP), the VOL, or the diameter of the lens at the equatorial plane (DIA). Accurate estimation of the EPP is very useful to predict where an intraocular lens (IOL) will be placed after cataract surgery (Estimated Lens Position, ELP), and thus of great value in the selection of the IOL power to be implanted in a patient. 


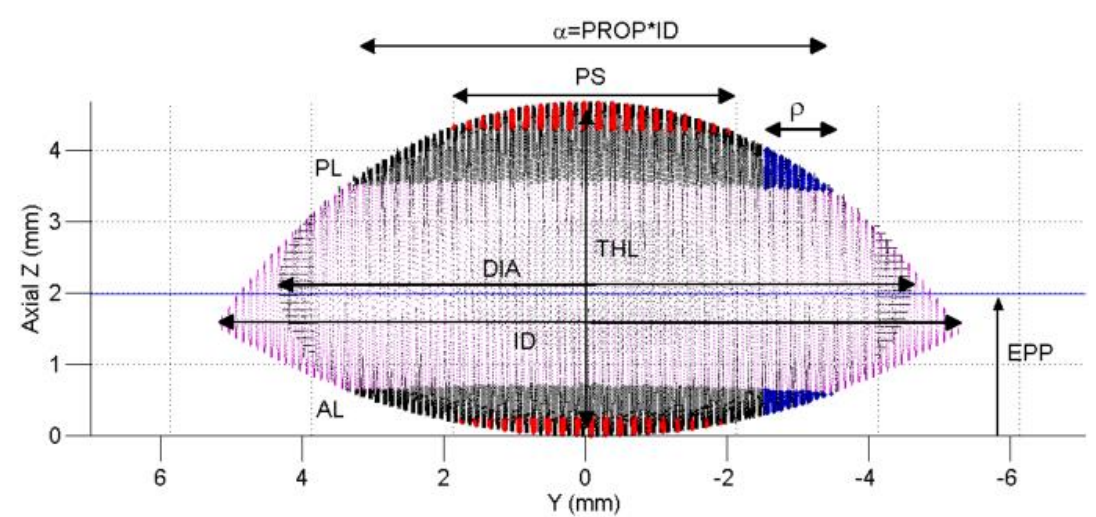

Figure 1.6 Lens model and definition of some parameters of interest. DIA: Diameter; PS: Pupil size; ID: Diameter in the intersection; AL: Anterior lens; PL: Posterior lens; EPP: Equatorial plane position; $\alpha$ : Diameter that defines the central portion of the lens; PROP: Proportion of the diameter in the intersection. $\rho$ : Part of the central portion taken to fit every lens side. (E. Martínez-Enriquez 2016)

\subsubsection{Iris, Pupil and Ocular biomechanics}

The iris is responsible for controlling the size of the pupil affected by the light conditions. The peripheral edge of the iris (its root) is merged with the ciliary body and the trabecular meshwork. The iris is $12-12.5 \mathrm{~mm}$ in diameter with a circumference of 36-37 $\mathrm{mm}$. In infants, the pupil diameter is minimal (up to $2 \mathrm{~mm}$ ), at a young age the pupil diameter is maximal (about $8 \mathrm{~mm}$ ), and in old age the pupil diameter decreases again which is referred to as senile miosis. In optical terms, the pupil serves as an aperture stop in eye, the main light-limiting aperture for on-axis objects. The iris also determines the depth of field of the whole eye. The chief rays go through the centre of the entrance pupil and define the centre of the blur in the presence of defocus. The size of the iris opening also has an effect on image sharpness and depth of field, a narrow pupil size reduces the effective lens opening surface for off-axis incident light in order to have a gradual decrease in light intensity towards the image periphery lens opening surface (Atchison DA 2000). Under the action of various eye muscles, the diameter of the pupil can be made narrower or wider. In conjunction with the ciliary muscles, the sphincter papillae muscle and dilator papillae control the size of the pupil. On the outer surface of the eye, except for the cornea, is the sclera, which protects the more delicate internal components of the eye, such as the lens, from external injuries and provides mechanical support to the retina.

This mechanism provides the eye with a continuously variable focusing system, which is controlled by a defocusing signal from the retinal image. This physic-optical system relies on the ability of the crystalline lens to deform elastically under zonular tension and to return to its more curved shape when zonular tension is relaxed. Biomechanics of eye muscles allow the eye to move extremely fast and engage in smooth pursuit to follow a moving object. The eyes also converge and diverge as well-moving toward or away from each other to maintain binocular vision with muscles and tissue that surround the eye to control all of these movements. 


\subsubsection{Pupillary axis, Visual axis and Fovea}

The optical surfaces of the eye are not rotationally symmetric. Consequently, the eye does not have a uniquely defined, true optical axis. The optical axis (OA) (blue line in Figure 1.7) is approximated by the line that best passes through or near to the centers of curvature of the four optical surfaces (anterior cornea, posterior cornea, anterior lens, posterior lens) of the eye while the visual axis (VA) (green line in Figure 1.7) is defined by the line connecting the fovea to the fixation target via the eye's nodal points. A line from the object through the centre of the entrance pupil that reaches the fovea (chief ray) is called line of sight (LOS) (orange line in Figure 1.7). The LOS defines the path of the principal ray entering the fixating eye. The pupillary axis (PA) (brown line in Figure 1.7) is defined by the line connecting the centre of the entrance pupil with the centre of curvature of the anterior cornea, and it therefore intersects the anterior cornea perpendicularly.

In Figure 1.7, the angle $\boldsymbol{\alpha}$ is formed at the first nodal point by the OA and VA, and typically has an average temporal decentration of 5 degrees (i.e. the fovea is shifted from the optical axis to the temporal retina) (Bradley. A and Thibos. 1995). VA is usual nasal to $\mathrm{OA}$ in a range of 17 degrees (nasal object space) to 2 degrees (temporal object space) is normal (Bradley. A and Thibos. 1995) and vertically $\alpha$ is declined 2 to 3 degrees (David A Atchison 2000, Sheehan 2012).

The angle $\boldsymbol{\kappa}$ is the angle between the PA and VA, which is easily observed as a displacement of the coaxially viewed corneal reflex from the pupil centre of a fixating eye. Hashemi et al. (Hashemi, KhabazKhoob et al. 2010) determined the mean angle kappa, which was $5.46 \pm 1.33^{\circ}$ in total; $5.41 \pm 1.32^{\circ}$ (male) and $5.49 \pm 1.34^{\circ}$ (female) $(\mathrm{p}=0.558)$, decreasing significantly with age; $0.015^{\circ}$ year $(\mathrm{p}<0.001)$.

The angle $\lambda$ is the angle between PA and LOS, with average values of 1.4 degrees (Loper 1959) and 9 degrees (Noorden. 1985) in the vertical and horizontal direction respectively, where the PA is temporal to the LOS in object space as conventionally defined (David A Atchison 2000). Rosales et al. reported that the off-axis field angle that would produce the measured shift of the first Purkinje image (More detail will be described in Section 2.1.3) is obtained by virtually rotating the eye using Phillips' linear equations. This angle is in reference to the PA and can be treated as angle $\lambda$ (between the LOS and PA) under the assumption that pupil decentration is small (Mandell 1995), with an average value of 0.36 degrees in horizontal axis and 3.6 degrees in vertical axis (Rosales and Marcos 2007). 


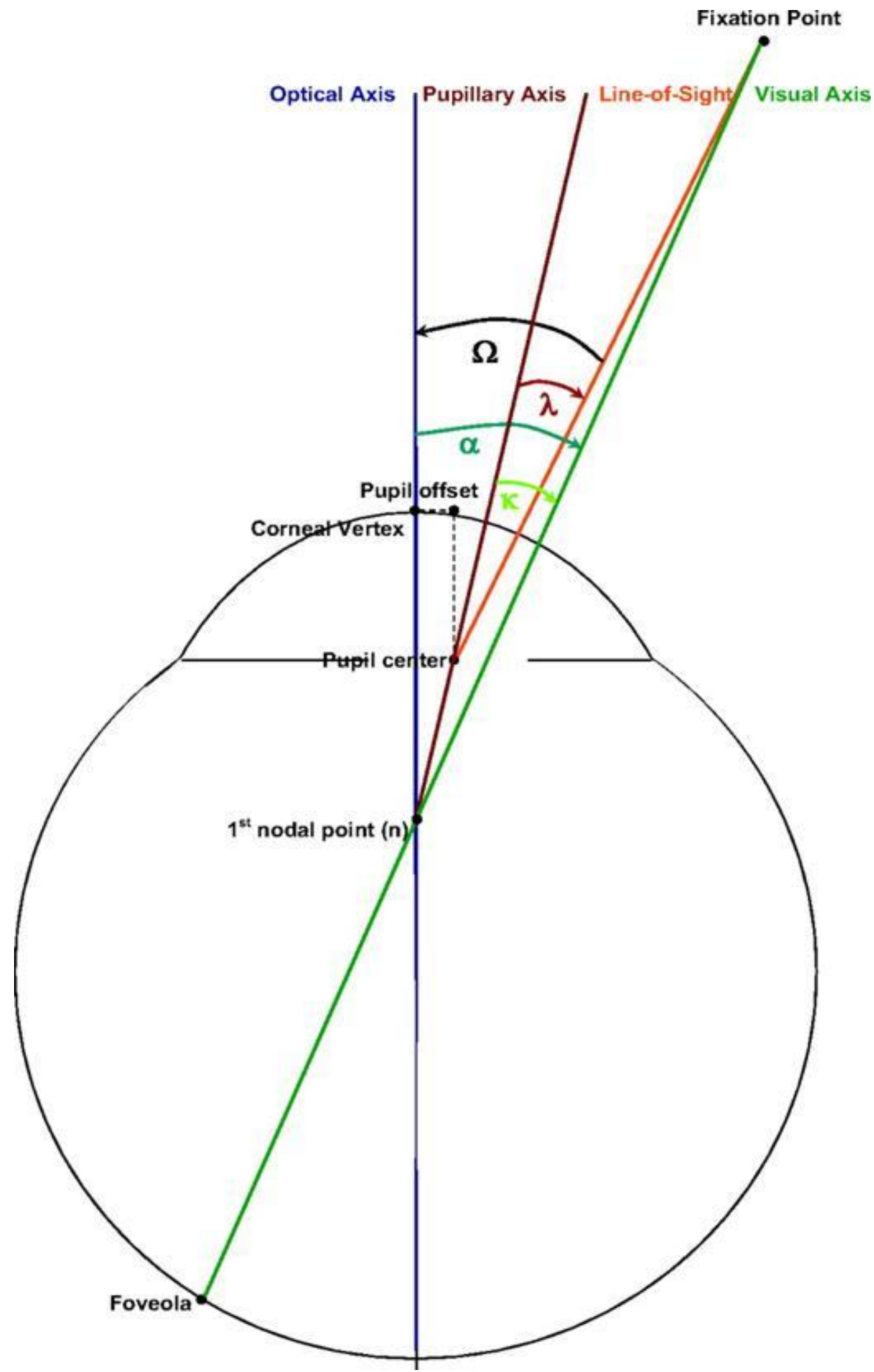

Figure 1.7 Drawn definitions of optical axis, pupillary axis, line of sight, visual axis (Arbelaez, Vidal et al. 2008)

Axes defined by fixation on a target are dependent on the target distance (which may be finite or infinite) and the refractive state of the eye. Apart from typically being decentered (to a much greater extent horizontally than vertically), the pupil is not static. Asymmetric dilation and constriction cause actuations in the location of axes defined by the entrance 
pupil center. Although the definitions of the visual axis and line of sight both contain the fixation target as a reference point, the object distance of the fixation target is not required to be infinite.

The optic nerve leaves the eye at the optic disc, which is a blind spot. The optic disc is about 5 degrees wide and 7 degrees high and is located about 15 degrees nasally on the fovea. The fovea is normally positioned below the optic nerve head, which might cause asymmetry in the distribution of the Retinal Nerve Fiber Layer (RNFL) between the superior and inferior retina (Hood, Raza et al. 2013), and in general, the fovea is displaced temporally and slightly inferior to the intersection of the optical axis with the retina and also with high intersubject variations in the position (James T. Schwiegerling 2013).

\subsubsection{Refractive errors}

The WHO estimates that 153 million people worldwide live with visual impairment due to uncorrected refractive errors. Refractive error (in Figure 1.8) is as an error due to the mismatch between the eye length and the optical power. Refractive errors usually can be "corrected" with eyeglasses or contact lenses, or they can be permanently treated with LASIK and other vision correction surgeries (also called refractive surgery). The most common types of refractive errors are myopia, hyperopia, presbyopia, and astigmatism. Myopia is a condition where the eye is longer or more powerful than normal and the rays of light come to focus in front of the retina, causing vision to be blurred. Myopia can be corrected, to allow a clear image of distant objects, by viewing through an appropriate negative power lens. Hypermetropia or hyperopia means that the eye is shorter or less powerful than normal and the rays of light come to focus behind the eye. Presbyopia leads to difficulty in reading or seeing at arm's length and it is linked to aging and occurs almost universally.

Astigmatism is caused by the front surface of the eye having two different radii of curvature along the horizontal and vertical axes (being shaped more like a rugby ball rather than a soccer ball), causing the rays of light to focus at different points. Astigmatism usually causes vision to be blurred or distorted to some degree at all distances. Astigmatism usually is caused by an irregularly shaped cornea as we mentioned in Section 1.2.2. The steepest and flattest meridians of an eye with astigmatism are called the principal meridians. In some cases, astigmatism is caused by the shape of the lens inside the eye. This is called lenticular astigmatism, to differentiate it from the more common corneal astigmatism. The power of the eye changes with meridian, due to one or more refracting surfaces having a toroidal shape, usually, or surface decentration or tilting. 


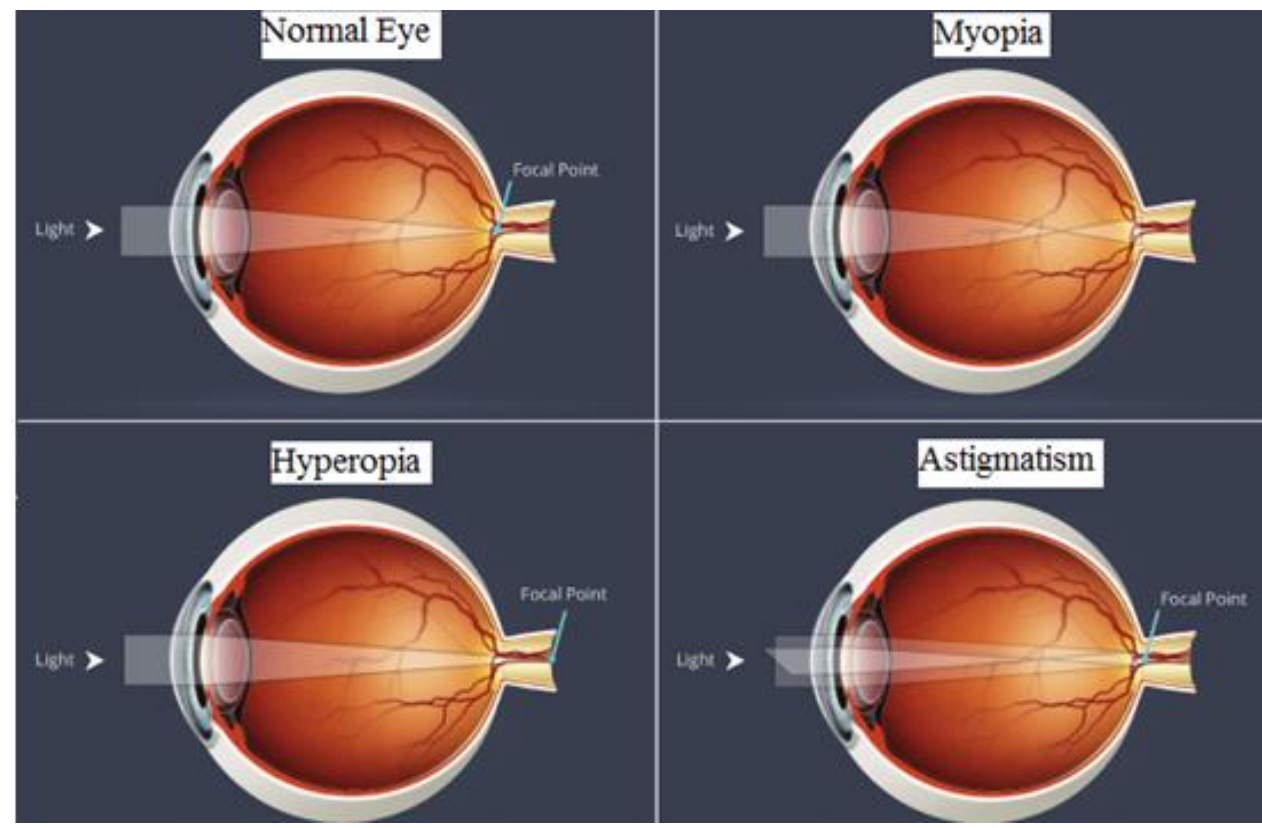

Figure 1.8 Diagrams of refractive error (Normal Eye, Myopia, Hyperopia, and Astigmatism)

\subsubsection{Optical aberrations}

The human eye is far from being a perfect optical system, as the images produced by the eye are affected by optical aberrations. Historically, measurements of aberrations in the human eye were restricted to the assessment of defocus and astigmatism, i.e. low order aberrations that can be corrected by conventional spectacles. In the $17^{\text {th }}$ century, Johannes Kepler described the use of spherical lenses to correct myopic and hyperopic refractive errors with a full ray tracing model (Kepler 1604). In addition to the usual refractive errors, the human eye suffers from high-order aberrations (HOA) like coma, trefoil, spherical aberration and others, which can produce vision errors such as difficulty seeing at night, glare, halos, blurring, starburst patterns or double vision (diplopia), shown in Figure 1.9. A HOA is a distortion acquired by a wavefront of light when it passes through an eye with irregularities in its refractive components (tear film, cornea, aqueous humor, crystalline lens and vitreous humor). The reduction in pupil size acts as an influence to moderate higher order aberration.

Visual field aberrations lead to rapidly increasing blur of the image with the angle of the visual field, strongly limiting the focusing capability of incident light in the periphery of the retina. It has not been until the last decades with the rapid increase of aberration measurement technology that measurement of HOA has been possible. In addition to aberrometry of the global eye, accurate measurement of the corneal shape has led to the first detailed analysis of the optical contributions of the different ocular elements, which opens a wide range of basic and clinical applications. One of the most useful applications of these technologies occurs in corneal laser surgery and cataract surgery. Measurement of the increase of aberrations induced by these surgeries has revealed issues that cannot be accounted for using classical refraction measurements. Furthermore, these types of measurements have opened the possibility of improving surgery by customization of laser 
ablation algorithms in corneal surgery or by optimizing the design of the intraocular lenses implanted in cataract surgery. Moreover, new technologies to correct eye aberrations have arisen with two fundamental goals: the improvement of visual quality and the increase of resolution of retinal imaging devices.

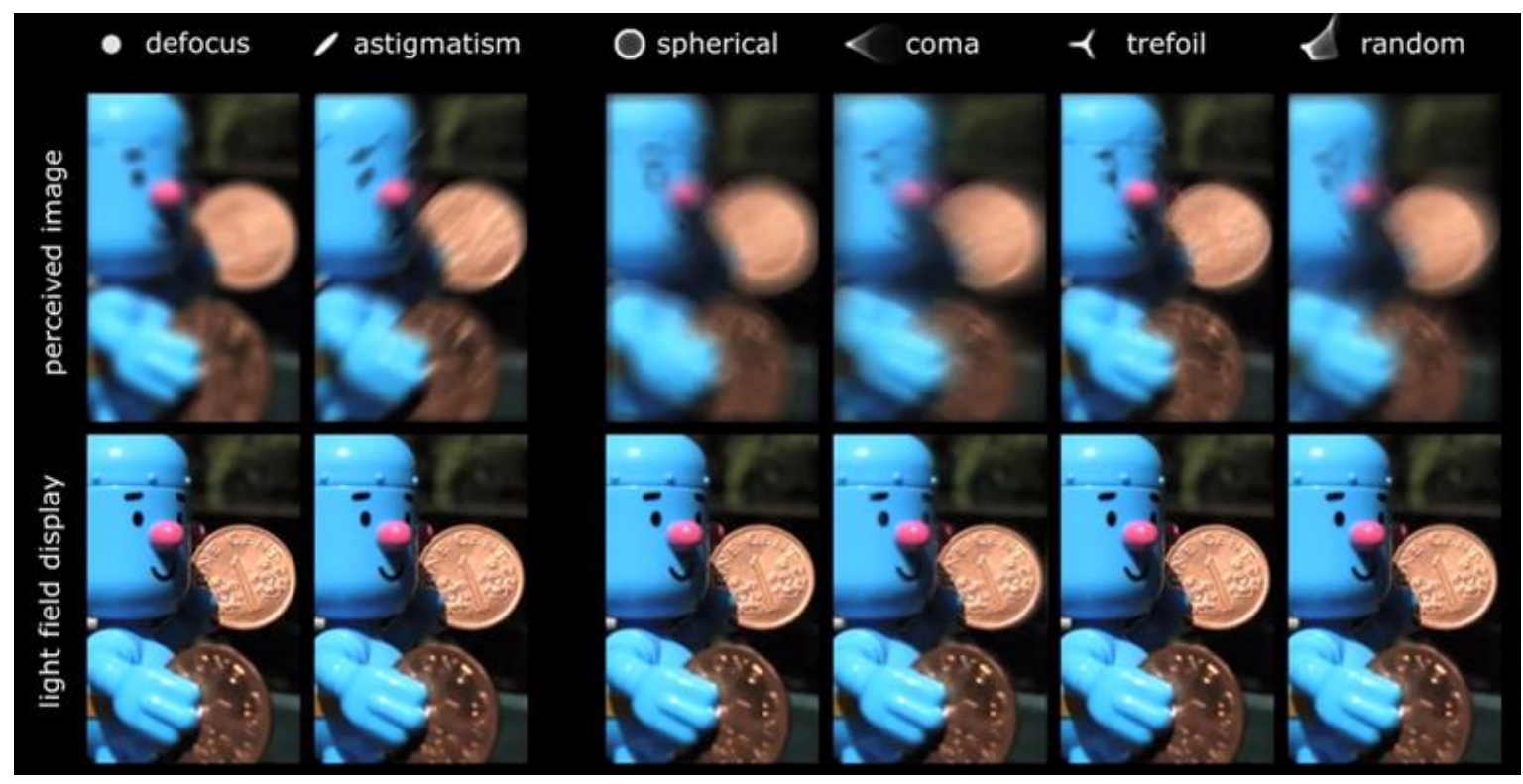

Figure 1.9 Perceived image with defocus, astigmatism, and high order aberration such as spherical aberration, coma, trefoil and random aberration vs. light field display (source: http://www.zdnet.com/article/researchers-eye-vision-correcting-displays-for-devices/)

Besides defocus, astigmatism is one of the most frequent, and important, aberrations of the eye (Vitale, Ellwein et al. 2008), followed by coma (Howland and Howland 1977, CastejonMochon, Lopez-Gil et al. 2002, Thibos, Hong et al. 2002). Apart from the natural astigmatism and coma that can be present in an eye on-axis, astigmatism and coma increases off-axis (Navarro, Moreno et al. 1998, Gustafsson, Terenius et al. 2001, Charman 2008). Off-axis aberrations become significant at large pupil diameters. Mathur et al. (Mathur, Atchison et al. 2009) indicate that off-axis eye defocus tends to be myopic, with the image field focusing away from the retina and toward the crystalline lens. Off-axis defocus of the eye effectively induces random asymmetrical deviations in field curvature, which may be noticeable and next to impossible to correct, either by crystalline lens refocusing or accommodation. In addition, the common axial (or central) astigmatic human eye also suffers from off-axis astigmatism, rapidly increasing with the distance from fovea (called retinal eccentricity). At large incident angles, the wavefront transforms from circular to strongly ellipsoidal, and the apparent field of view effectively shrinks due to significant refraction angles of the chief ray at the cornea and lens. Most research data indicate that off-axis astigmatism in the average eye tends to be larger on the nasal side of the retina. Astigmatism here initially diminishes outside the fovea and well into the macula, dropping to zero at about $8^{\circ}$ and $14^{\circ}$ temporally and nasally, respectively, due to the offset of axial eye astigmatism with that caused by oblique incident rays. Off axis astigmatism also changes with accommodation level, not only in magnitude but also in form, with the field asymmetry, possibly by shifting from temporal to nasal dominance (Lee, Thompson et al. 2010). The success of different correction alternatives for myopia relies on an adequate knowledge of the optics of the myopic eye. Several studies show an increase of 
the amount of higher order aberrations in myopic eyes (Collins, Wildsoet et al. 1995, Goss, Van Veen et al. 1997), while a certain degree of corneal/internal spherical aberration balance seems to hold in a wide range of refractive errors from 0D to -16 D (Marcos, Rosales et al. 2008). The increase of corneal spherical aberration relates with increased corneal asphericity found in myopia (Carney, Mainstone et al. 1997). In the last few years there has been an increased interest in the assessment of the optical quality of the normal eye (Marcos, Burns et al. 2001, Castejon-Mochon, Lopez-Gil et al. 2002), as well as the changes of optical quality under certain conditions such as aging (McLellan, Marcos et al. 2001), accommodation (He, Burns et al. 2000) and refractive errors (Llorente, Barbero et al. 2004), particularly how the optical aberrations are modified after certain interventions such as refractive surgery (Moreno-Barriuso, Merayo-Lloves et al. 2001), intraocular surgery (Barbero, Marcos et al. 2003) and contact lenses (Dorronsoro, Barbero et al. 2003). 


\subsection{The aging eye}

\subsubsection{Presbyopia: accommodation loss of the crystalline lens}

The anterior and bulk of the ciliary body contains the ciliary muscle that affects zonules in the eye (Figure 1.10), enabling changes in lens shape for light focusing (Atchison 1995) and in turn controls accommodation. For the elastic young crystalline lens without presbyopia or cataracts, this dynamic process of contraction and relaxation of the ciliary muscle adjusts the focusing power of the eye by changing the thickness of the eye's natural lens and shape via constriction and/or relaxation of the ciliary muscle, resulting in a change of the optical power of the eye which provides a focused image of objects both near and far. With aging, the human lens becomes thicker, relatively steeper, and looses the ability to accommodate (Atchison 1995, Glasser and Campbell 1998, Michael and Bron 2011). Presbyopia is an age-related eye condition affecting $100 \%$ of the population beyond 45 years, characterized by the stiffening of the crystalline lens, resulting in the loss of its ability to accommodate near and far (Lewis 1983, Koretz, Cook et al. 1997, Glasser and Campbell 1999, Glasser, Croft et al. 2001, Spierer 2003, Strenk 2005). The accommodation capacity is also lost with age as a natural part of the aging process (Pascolini, Mariotti et al. 2004, Resnikoff, Pascolini et al. 2004, Resnikoff, Pascolini et al. 2008) due to lens stiffening. The ocular changes undergone in presbyopia have been studied from physiological (Koretz, Handelman et al. 1984, Koretz, Kaufman et al. 1989, Glasser and Campbell 1998, Glasser and Campbell 1999, Koretz, Cook et al. 2001, Koretz, Cook et al. 2002, Moffat, Atchison et al. 2002, Strenk, Strenk et al. 2004, Dubbelman, Van der Heijde et al. 2005, Kasthurirangan 2007), anatomical (Croft, Glasser et al. 2001, Glasser, Croft et al. 2001), biomechanical (Burd, Judge et al. 1999, Judge and Burd 2002, Hermans, Dubbelman et al. 2006, Weeber and van der Heijde 2007), and biochemical approaches (Bron, Vrensen et al. 2000, McGinty and Truscott 2006) although there is ample consensus that stiffness in the lens is the cause for presbyopia, some authors have also invoked age-related changes in the ciliary muscle and capsular bag (Kaufman, Bito et al. 1982, Glasser, Croft et al. 2001, Croft, McDonald et al. 2009).

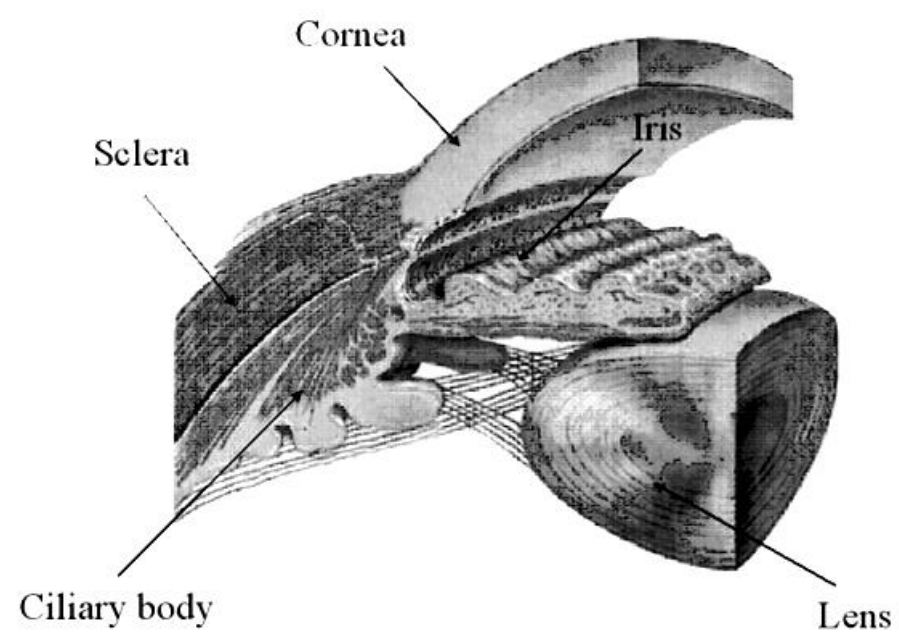

Figure 1.10 A quarter section through the anterior segment 
To relieve the symptoms of presbyopia, it is necessary to understand the accommodative anatomy, the mechanism of accommodation and the causes of presbyopia. A variety of surgical procedures have been considered for restoring accommodation to the presbyopic eye or at least to produce some visual functionality at near and far distances. Non-surgical treatments may come in the form of alternating vision (near spectacles or progressive spectacles), mono-vision (correcting an eye for far and the other for near) or simultaneous vision (multifocal contact lenses, corneal inlays, presbyLASIK or multifocal IOLs). Other treatments with dubious effectivity include surgical scleral expansion or femtosecond laser lens treatment. Accommodating IOLs aim at restoring dynamic accommodation, being provided with a mechanism to change their optical power activated by the forces of the ciliary muscle. Several general designs are being considered: single element IOLs that rely on a forward shift of the optic, dual optic IOLs that rely on an increased separation between the two optical elements either laterally or axially, and IOLs which permit a change in surface curvature to produce an increase in optical power in response to ciliary muscle contraction (Glasser 2008). Of those, only a single optic IOL, the Crystalens by B\&L, is FDA approved, although its results have been questioned (Marcos, Ortiz et al. 2014).

Improved solutions of presbyopia, for example in the form of accommodative IOLs, require a profound understanding of the natural crystalline lens properties and their contribution to accommodation. Furthermore, new refractive products and, more specifically, new IOL options hold promise for a customized intervention, adapted to the patient's needs, but critically rely on a good tuning with respect to other elements in the eye. There is an increasing number of IOLs aimed at modifying the patient's wave aberrations, targeting correction of both refractive error and some HOA (i.e. spherical aberration, such as in aspheric IOLs, or aiming at the increase of depth-of-focus in presbyopic-correction IOLs). The ultimate goal in IOL development is to restore the active change in power of the eye that occurs during accommodation in the young eye. More types of IOLs will be introduced in Section 1.5.2.

\subsubsection{Cataract: transparency loss of the crystalline lens and clinical solutions}

The Crystalline lens is mostly made of water and protein, with the protein arranged in a precise way that keeps the lens clear and lets light pass through it. As we age, some of the protein may clump together and start to cloud a small area of the lens (Benedek 1971). Over time, it may grow larger and cloud more of the lens, making it harder to see. This clouding of the lens, the cataract, creates vision loss, not allowing the image to focus properly on the retina. There are three main types of Cataracts: Subcapsular cataract, which occurs at the back of the lens and is more prevalent in cases of diabetes or with high doses of steroid medications; Nuclear cataracts form deep in the central zone (nucleus) of the lens, which are associated with aging (Michael and Bron 2011); Cortical cataracts are characterized by white, wedge-like opacities that start in the periphery of the lens and spread to the lens cortex, which is the part of the lens that surrounds the central nucleus (Truscott and Zhu 2010).

The first cataract surgery was performed in the $5^{\text {th }}$ century BC in Egypt. The removal of cataracts by surgery was also introduced into China from India, and flourished in the Sui 
(AD 581-618) and Tang dynasties (AD 618-907) (Deshpande 1999, Deshpande 2000). Cataract surgery can generally be distinguished by three techniques: Extra capsular cataract extraction (ECCE), Intracapsular cataract extraction (ICCE) and Phacoemulsification. The first extracapsular extraction of a cataract was performed by French surgeon Jacques Daviel in Paris in 1748 (Era. 2008). ICCE was developed a short time later in 1752-1753, but it is difficult to recover sight after ICCE, regardless of whether or not an IOL is implanted, particularly because of the size of the incision required. When removing cataracts from eyes, ICCE additionally removes capsular barriers and intraocular lens implantation is limited to the iris support, angle support, or sulcus support design. ICCE is seldom used today because more advanced techniques are available.

The most significant change in cataract surgery marked by the modern era was the introduction of phacoemulsification surgery in 1967 by Dr. Charles Kelman (Linebarger, Hardten et al. 1999). Since then, cataract surgery with IOL implantation has become a routine surgical procedure. With modern techniques, complications are uncommon and increasing emphasis is being placed on refractive outcomes. The key to reducing incision size lies in the development of the Phacoemulsification extraction technique and the foldable IOL. Today, Phacoemulsification cataract extraction (shown in Figure 1.11) allows most of the capsule to be preserved and a foldable IOL can be inserted into the capsular bag without widening the corneal incision, which means that incisions are typically less than $3.5 \mathrm{~mm}$, are configured to self-seal without the need for sutures and do not induce significant astigmatism (Long and Monica 1996). Phacoemulsification with in-the-bag implantation combined with modern surgical technologies has resulted in a refined surgical procedure, which is highly repetitive and highly replicated worldwide. An increase in visual acuity is the primary aim of cataract extraction when the crystalline lens is removed in cataract surgery with increasing patient expectations and the ability of surgeons to control astigmatism induction using small-incision techniques and/or toric IOLs, the refractive outcome has become increasingly important (Kershner 1997, Kohnen and Koch 1998, Koch and Kohnen 1999, Mamalis 2003).

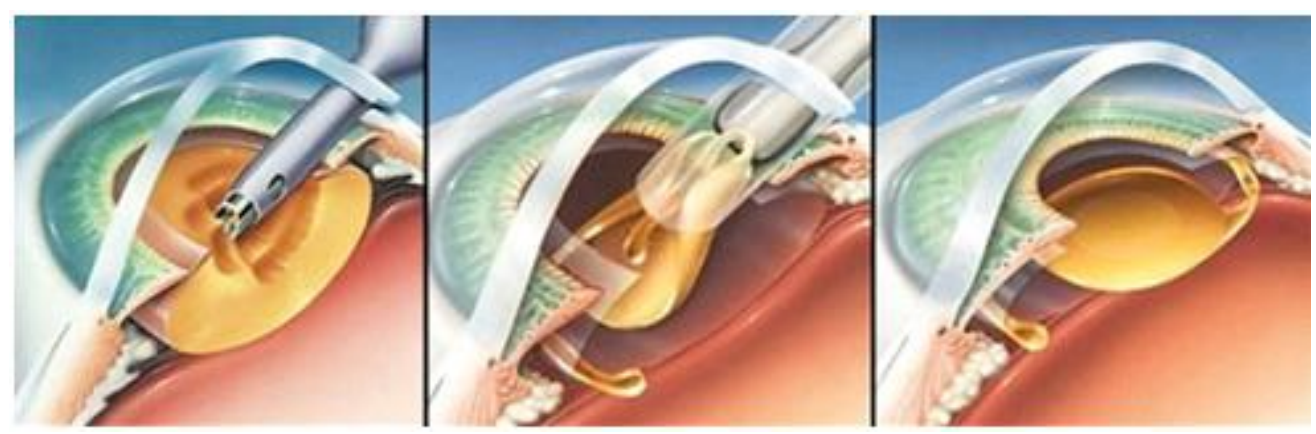

Figure 1.11 The modern technique of Phacoemulsification which consists in the emulsification (dissolve and aspire) of the crystalline lens inside the eye and the introduction of a foldable lens through an average 2 -mm incision.

Although the main goal of a cataract treatment was originally to eliminate scattering, IOLs have evolved enormously, first to additionally correct for refractive errors, but more recently, to compensate for HOA, particularly spherical aberration. Future IOL design is intended to provide enhanced functionality, such as regulatory function, chromatic aberration correction and monochromatic aberration correction. 
An excessive anterior capsule fibrosis/opacification (ACO) may result in significant clinical problems and complications, such as checking the retinal periphery, which leads to difficulty in diagnosing and treating retinal problems and also lead to fibrous contraction of the capsule, capsulorhexis phimosis, and IOL decentration. The technical and surgical advances have not been in general paralled by a sophistication in the IOL power selection, which remains still empirical and based on regressions from pooled data in patients.

\subsubsection{IOL power calculation}

In 1950s, Harold Ridley implanted the first IOL and formulas for calculation of IOL power have been evolving since their inception. Fyoderov and co-workers first estimated the optical power of an IOL using vergence formulas in 1967 in Russia (Fedorov SN 1967). In the 1970s, several studies were conducted to establish and publish various theoretical vergence formulas. The theoretical formulas have been subjected to minor and major alterations in the variables with immersion A-scan since then in order to increase accuracy (Hoffer 1993). In the 1980s, many ways were looked for to improve IOL calculations because of inaccuracies using applanation ultrasound. This led to the development of regression formulas, which soon coalesced into the SRK formula, which was developed by Sanders, Retzlaff and Kraff. Though now replaced with the newer generation formula, it is still useful for understanding the relation of the variables and A-constant to the IOL Power (P). $\mathrm{P}=\mathrm{A}-0.9 \mathrm{~K}-2.5 \mathrm{AL}$. Later in 1990, Holladay improved the prediction of the estimated lens position (ELP), where it can be assumed that size and position of the crystalline lens have a significant predictive impact on postoperative ACD, and helped lead many back to the use of theoretic formulas (Holladay, Prager et al. 1988). This led to the development of the SRK/T formulas (a combination of a linear regression method and a theoretical eye model) (Retzlaff, Sanders et al. 1990) by Retzlaff, the Hoffer Q formula (Hoffer 1993), Olsen formula (Olsen T 2004) and the Haigis formula (Haigis W. 2004) in 2000. These can be classified on the basis of their derivation (theoretical, regression analysis or combination) or according to their evolution as per generations. The accuracy of the results led to a standard of aiming for emmetropia in all cataract patients, so the calculation of IOL power is not absolute due to the large number of individual variations in human eyes. Theoretical formulas are determined by application of geometrical optics to the schematic and reduced eyes using various constants by derivation.

Studies based on preoperative and postoperative ultrasound biometry show that over $50 \%$ of errors in predicted refraction after IOL implantation can be attributed to axial length (AL ) measurement errors, around $40 \%$ to incorrect estimation of postoperative anterior chamber depth (ACD), and around 10\% to corneal power (K) measurement errors (Olsen 1992). Therefore, AL measurement and calculation of IOL power by optical biometry should be comparable or more accurate than those obtained using applanation ultrasound in the normal population (Drexler, Findl et al. 1998, Findl, Kriechbaum et al. 2003, Findl 2005). More recent commercial optical instruments for AL measurement are the IOL master (Zeiss Meditec Jena, Germany) with the advantages of laser optical biometry in a noncontact technique, and Lenstar LS 900 (Haag-sreit Koeniz, Switzerland) with high accuracy and repeatability. More information of ocular imaging techniques will be introduced in the Section 1.4. 
The assumed optical biometry, optimized IOL constants and the accuracy of predicted postoperative IOL position are the limiting factors of modern IOL power calculation formulas today. Several studies have proven that formula personalization definitely improves formula accuracy in a clinically significant way. The customized pseudophakic computer eye models based on ray tracing applied to IOL calculation utilizes a description of the pseudophakic eye in which anterior and posterior corneal surfaces should ideally be topographically measured. The use of customized eye models overcomes all the limitations of the theoretical simple computations and allows understanding the contribution of each factor to the optical quality and to perform this evaluation on an individual basis. More information of customized eye model will be introduced in the Section 1.5. 


\subsection{Ocular imaging techniques}

Imaging of the eye is critical to improving the diagnosis, evaluating of management of eye disease, and observing changes in anatomy and physiology. The technology and clinical application of ocular imaging techniques, Scheimpflug imaging, ultrasonography, optical coherence tomography, confocal scanning microscopy and MRI are introduced in the following.

\subsubsection{Scheimpflug, ultrasonography, MRI, and Purkinje imaging system}

The Scheimpflug is configured to have the image, lens, and object plane intersect at one point so that sections of the eye appear with a large depth of focus (Brown 1972, Koretz, Handelman et al. 1984). The tilted film plane creates a significant variation in the magnitude of light in different parts of the image by the use of strong slit-beam illumination and a very sensitive film. Despite the large depth of focus of Scheimpflug imaging-based systems, which are capable of capturing cross-sections of the human eye from the anterior cornea to the posterior crystalline lens (particularly under full dilation) (Koretz, Handelman et al. 1984), current commercial instruments are mostly used as corneal topographers and pachymetry, discarding quantitative information that could be extracted on the crystalline lens geometry. However, Scheimpflug images suffer from geometric distortion (caused by tilt of the object plane with respect to the optical axis of the instrument) and optical distortion (caused by refraction from the different ocular surfaces). Subsequent studies of human accommodation and presbyopia using the Scheimpflug principle have provided a wealth of information about these processes, as well as data about adult human lens development and aging.

In ophthalmology, most A-scan and B-scan ultrasonography probes use a frequency of approximately 10 million $\mathrm{Hz}(10 \mathrm{MHz})$, which allows for the restricted depth of penetration of the sound into the ocular. The most common use of the A-scan ultrasonography (A-scan US) is to determine eye length for calculation of intraocular lens power, and it also provides distance measurements (e.g., anterior chamber depth, lens thickness). The horizontal and vertical sweeps generally applied to pulse echo ultrasonics are proportional to time or distance and amplitude or magnitude respectively. Thus the location and magnitude of acoustical interfaces are indicated as the depth below the transducer. However, A-scan US (Koretz, Kaufman et al. 1989, Beers and van der Heijde 1996, Garner and Smith 1997, Koretz, Strenk et al. 2004) is unable in providing information about some ocular components, such as crystalline lens shape and ciliary muscle orientation. Over the last 30 years, visualization is considered important to provide accurate prognosis for vision after cataract surgery. B-scan ultrasonography has greatly advanced, which helps in evaluating the posterior segment in the presence of opaque ocular media (Coleman, Jack et al. 1972) (John A. 2001), providing good quality real-time imaging. However B-scan US requires a high level of inter-operator variability (Chopdar 2014), which does not plague other forms of imaging including optical coherence tomography, CT, and MRI, and is limited by low sensitivity and specificity.

Standard magnetic resonance imaging (MRI) uses a magnetic field and pulses of radio wave energy to make images of ocular in ophthalmic field without requiring any 
assumptions concerning lens optical properties for dimensional measurements. Previous research reported overall eye shape, extraocular muscle anatomy, crystalline lens shape, refractive index distribution, and ciliary muscle anatomy using MRI for ocular imaging (Strenk, Strenk et al. 2000, Atchison and Smith 2004, Strenk, Strenk et al. 2004, Jones, Atchison et al. 2007, Atchison, Markwell et al. 2008, Demer, Clark et al. 2008, Kasthurirangan, Markwell et al. 2008). We have previously described the dependence of refractive index distribution of the crystalline lens on accommodation and age using MRI (Jones et al., 2007; Kasthurirangan et al., 2008). However, MRI is a relatively timeconsuming technique which makes the images prone to motion artifacts and of lower resolution compared to optical and ultrasound techniques.

Purkinje imaging relies on reflections from the anterior (PI) and posterior (PII, usually not visible) corneal surfaces and from the anterior (PIII) and posterior (PIV) lens surfaces with off-axis illumination to avoid overlapping of the images. Purkinje images have also been used to measure phakometry (Crystalline lens radii of curvature), (Mutti, Zadnik et al. 1992, Kirschkamp, Dunne et al. 2004, Dunne, Davies et al. 2005, Rosales, Dubbelman et al. 2006, Rosales and Marcos 2006), as well as to assess IOL tilt and decentration (Phillips, Perez-Emmanuelli et al. 1988, Auran, Koester et al. 1990, Tabernero, Piers et al. 2006, de Castro, Rosales et al. 2007, Rosales, De Castro et al. 2010) and Barry et al., (Barry, Dunne et al. 2001) proposed a set of linear equations relating the position of PI, PIII, and PIV as a function of linear combinations of eye rotation, IOL tilt, and IOL decentration. Until recently most reports of IOL tilt and decentration were obtained by presenting to the subject fixation targets at different eccentricities and determining the fixation angle that produces an overlap of Purkinje reflections from the anterior and posterior lenses (Guyton, Uozato et al. 1990).

\subsubsection{Optical Coherence Tomography (OCT)}

Optical Coherence Tomography (OCT) is a three dimensional imaging technique with ultrahigh spatial resolution even in high scattering media and is one of the most rapidly developing biomedical imaging modalities. It is based on measurements of the reflected light from tissue discontinuities to create interference with a reference beam. The first biological application of low-coherence interferometry was reported by Fercher et al. in 1988 for measuring the eye axial length (Fercher, Mengedoht et al. 1988). Further developed in 1997 in Prof. James Fujimoto's lab at MIT, OCT with micrometer resolution and cross-sectional imaging capabilities has become a prominent biomedical tissueimaging technique and it is particularly suited to ophthalmic applications requiring micrometer resolution and millimeter penetration depth (Tearney, Brezinski et al. 1997).

The most important OCT modalities include: Time-Domain OCT (TD-OCT) and Fourierdomain OCT (FD-OCT). In Time-Domain OCT (Figure 1.12(a)), the autocorrelation of the light field is measured directly by mechanical axial movement of a reference mirror, which corresponds to the depth-scanning signal of the sample (Huang, Swanson et al. 1991, Fercher, Hitzenberger et al. 1993, Swanson, Izatt et al. 1993, Izatt, Hee et al. 1994). Uhlhorn et al. (Uhlhorn, Borja et al. 2008) developed a TD-OCT with a long imaging depth of 10 $\mathrm{mm}$ in air for in vitro crystalline lens imaging in sequence over time, but low acquisitions speed is not suitable for in vivo dynamic studies with eye motion. Fourier-domain OCT 
is rapidly replacing the TD-OCT in most applications due to significant advantages in sensitivity and imaging speed. In FD-OCT, a tomogram line is calculated by unevenly sampled Fourier transformation of the recorded fringe pattern, as demonstrated in 1995 by Fercher (Fercher 1995).

Both systems use a reference arm and an interferometer to detect echo time delays of light as shown in Figure 1.12. The interferometer uses a beam splitter, dividing the light into a measurement sample arm and a reference arm. The reference arm in TD-OCT is mechanically scanned (by a moving mirror) in order to produce a varying time delay. In the FD-OCT system, all echo delays are acquired simultaneously enabling a significant increase in the speed of image acquisition.

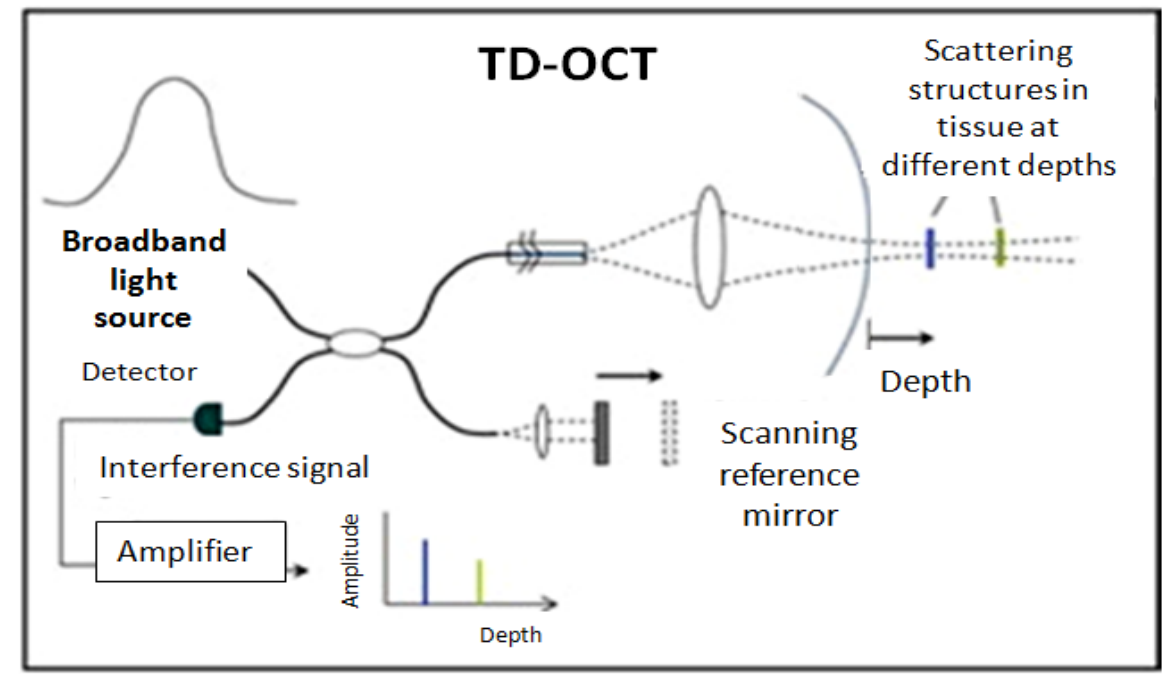

(a)

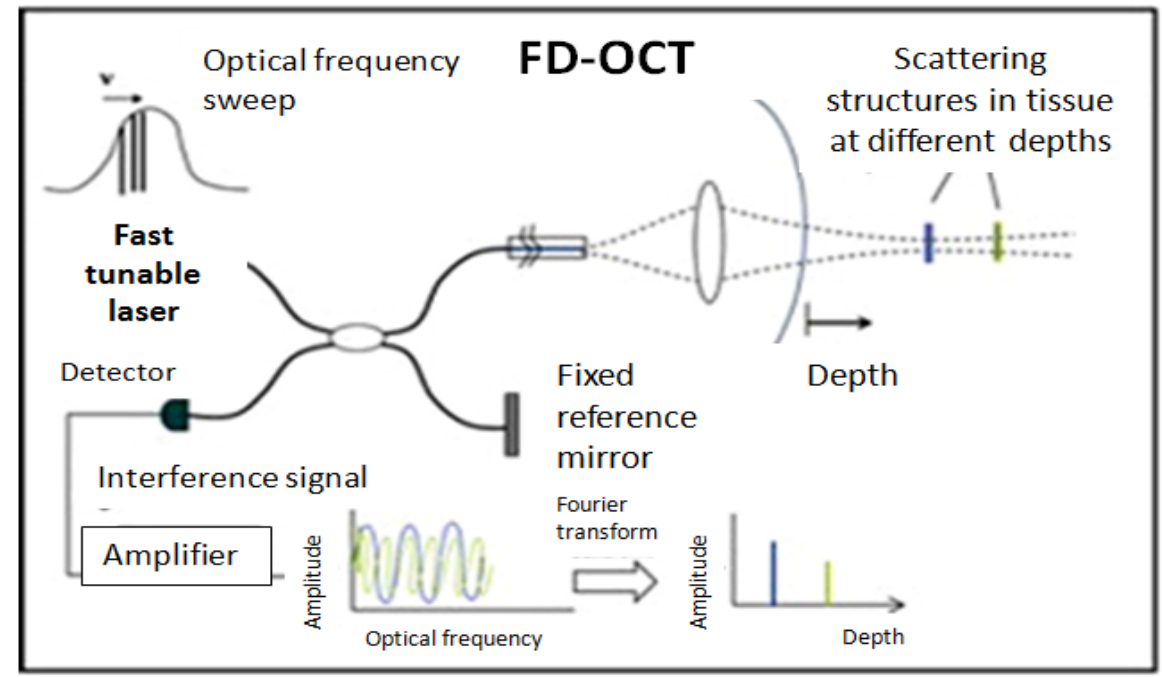

(b)

Figure 1.12 Schematic representations of (a) TD-OCT and (b) FD-OCT. (Bezerra, Costa et al. 2009)

Two configurations have prevailed in Fourier domain systems: spectral domain OCT (SDOCT), which uses a grating to spatially disperse the interference spectrum across an arraytype detector (Ha Usler and Lindner 1998, Wojtkowski, Bajraszewski et al. 2003, Park, 
Pierce et al. 2005), and swept source OCT (SS-OCT) where a narrow band laser is swept across a broad spectrum, encoding the spectrum as a function of time (Chinn, Swanson et al. 1997, Yun, Tearney et al. 2003, Choma 2005). FD-OCT employing wavelength tunable lasers (swept source OCT) exhibits improved imaging speed and detection sensitivity in comparison to traditional time domain detection techniques. (Choma, Sarunic et al. 2003, de Boer, Cense et al. 2003, Leitgeb, Hitzenberger et al. 2003).

SD-OCT is a non-invasive, high-speed technique that allows the collection of 3D anterior segment data in hundreds of milliseconds with high lateral resolution and axial resolution, which presents several advantages over other techniques to evaluate ocular anterior segment biometry. Another major step in the improvement of OCT imaging speed, which led to obtaining a value of 15,000 A-scan/s, was shown in 2003 by Wojtkowski et al (Baikoff 2006). Since then, several studies have reported different corneal parameters in normal and keratoconic subjects using OCT: central corneal thickness (Li, Meisler et al. 2008, Rao, Kumar et al. 2011), geometry of anterior and posterior corneal surfaces and corneal topography maps (Ortiz, Siedlecki et al. 2009, Ortiz, Siedlecki et al. 2010, Ortiz, Siedlecki et al. 2011, McNabb, Kuo et al. 2013). However, quantitative geometrical parameters can only be retrieved accurately upon correction of the fan distortion (or scanning distortion) of the sample arm of the OCT instrument and optical path difference (OPD) correction of the optical distortion (or refraction error) (Ortiz, Siedlecki et al. 2009, Ortiz, Siedlecki et al. 2009, Ortiz, Siedlecki et al. 2010, Zhao, Kuo et al. 2010, Ortiz, Siedlecki et al. 2011).

SS-OCT offers the required speed but the challenge of providing limited axial range if axial resolution is not to be compromised. The first three publications to report on the potential of swept lasers in OCT imaging were published in 1997 (Chinn, Swanson et al. 1997, Golubovic, Bouma et al. 1997, Lexer, Hitzenberger et al. 1997). The SS-OCT (shown as Figure 1.13(b)) is based on Wolf's solution to the inverse scattering problem of the Spectral Domain OCT, but instead of encoding the frequencies in space they are encoded in time (shown as Figure 1.13(a)). This overcomes the main drawbacks of SD-OCT, which are the sensitivity drop-off and the resolution imposed by the finite size of the pixels of the camera, which removes the contribution of the higher frequencies. The high imaging speed of SSOCT allows high-resolution images to be obtained while reducing the negative effect of a patient's eye movements on scan quality and also allows the vitreous and the choroid to be imaged simultaneously because of the deeper range afforded by SS-OCT. 
Spectral OCT

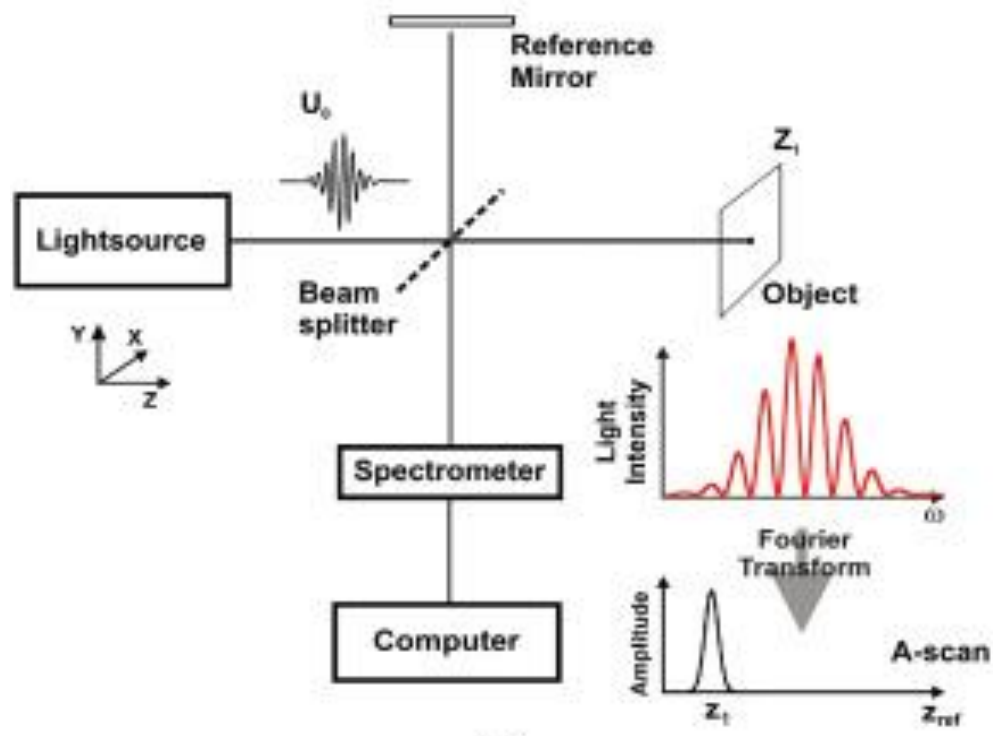

(a)

Swept Source OCT

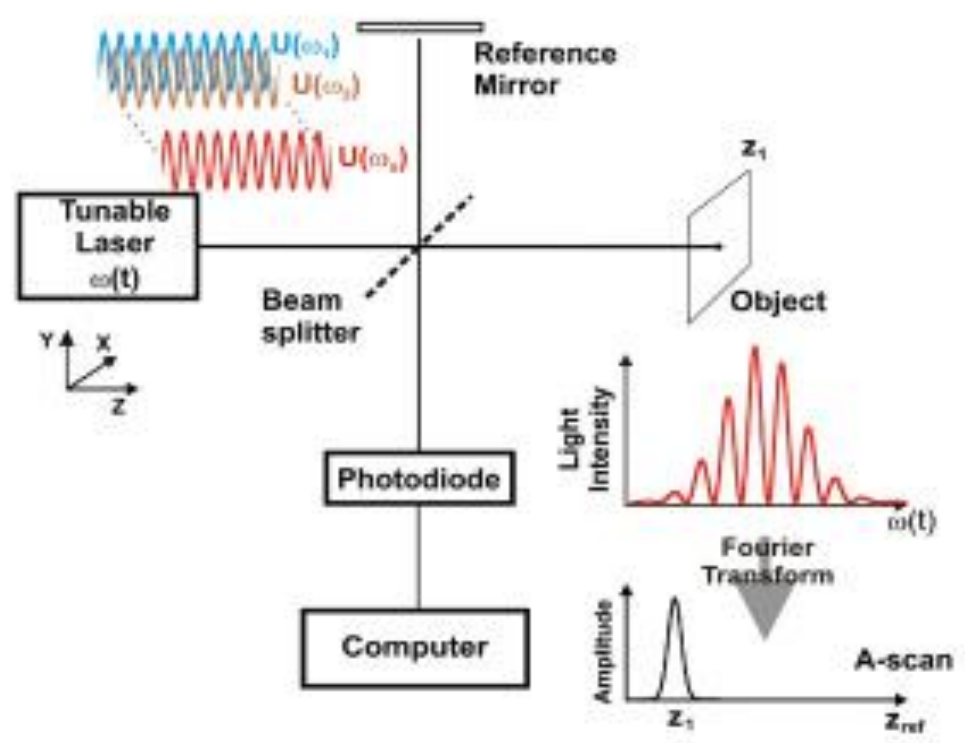

(b)

Figure 1.13 Block diagrams of the OCT methods with the use of Fourier-domain detection: (a) SD-OCT, (b) SS-OCT. (Wojtkowski 2010)

\subsubsection{Aberrometry}

Many scientists throughout the history of physiological optics have attempted to measure the optical aberrations of the human eye. One of the earliest quantitative aberrometers was devised by Tscherning et al. (Tscherning 1894, Arnulf 1956, Howland 1960, Smirnov 1961, Howland and Howland 1976, Howland and Howland 1977). Aberrometry techniques such as Hartmann-Shack systems or Laser Ray Tracing (LRT) have become the most relevant technologies to measure aberrations in the laboratory and in the clinic. Hartmann-Shack 
wavefront sensors $(\mathrm{H}-\mathrm{S})$ consist of an array of lenslets of the same focal length focusing onto a CCD camera, and are an objective 'outgoing' aberrometry technique (Hartmann 1900, Hartmann 1904, Shack 1971). The lenslet array creates spots in the sensor according to the wavefront coming out of the eye, as shown in Figure 1.14. The transverse ray aberration associated to each pixel can be determined from deviations from the focal points. H-S samples the pupil with a high fill factor and high-speed (milliseconds) which are the two main advantages over sequential techniques such as LRT.

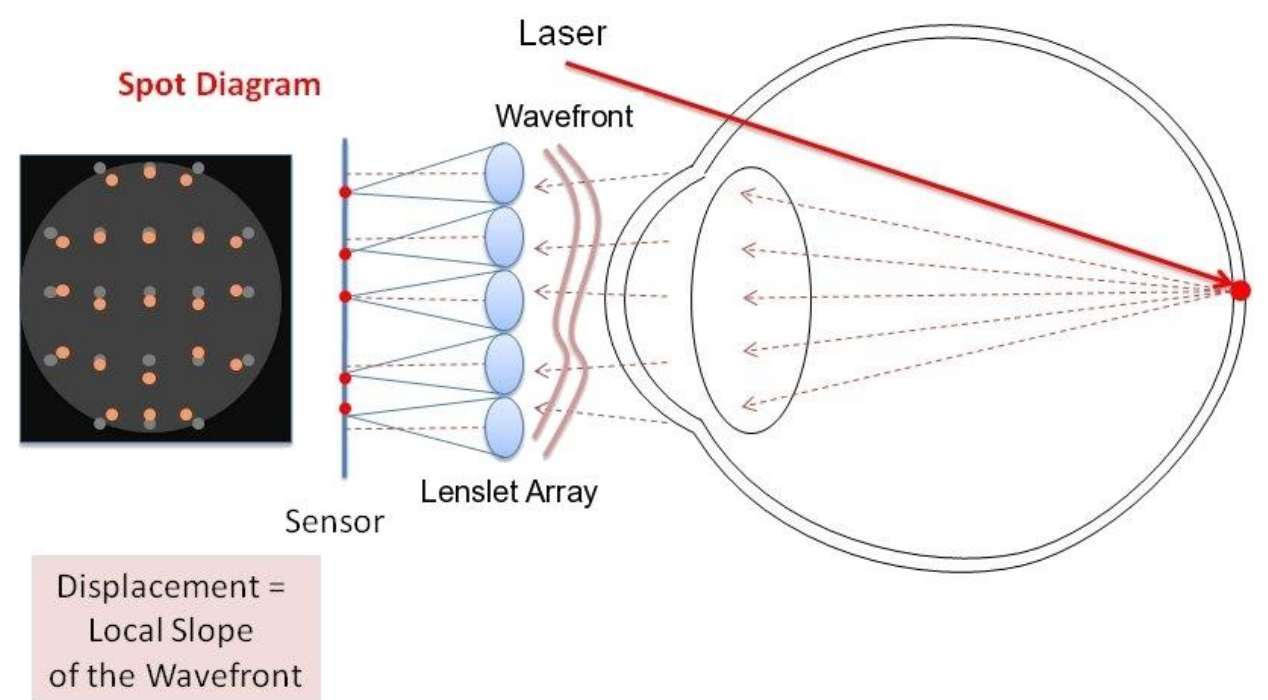

Figure 1.14 Hartmann-Shack system in optometry: a laser creates a virtual light source in the retina. The lenslet array creates spots in the sensor according to the wavefront coming out of the eye. The local tilt of the wavefront across each lens can be calculated from the position of the focal spot on the sensor. (Source: https://en.wikipedia.org/wiki/Shack\%E2\%80\%93Hartmann _wavefront_sensor)

There are two main differences between Hartmann-Shack (Figure 1.15(a)) and LRT (Figure 1.15(b)): H-S works in parallel with a fixed sampling pattern and measures the aberrations in the outgoing (second-pass) aerial beam, while LRT is sequential with a highly flexible sampling pattern and measures aberrations of the incoming beam (first-pass) at the retinal surface. 


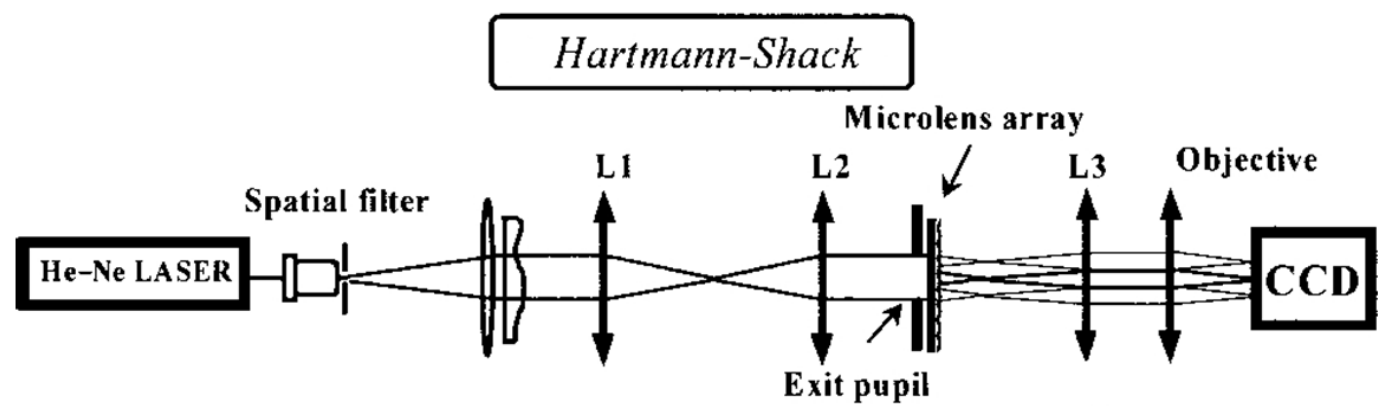

(a)

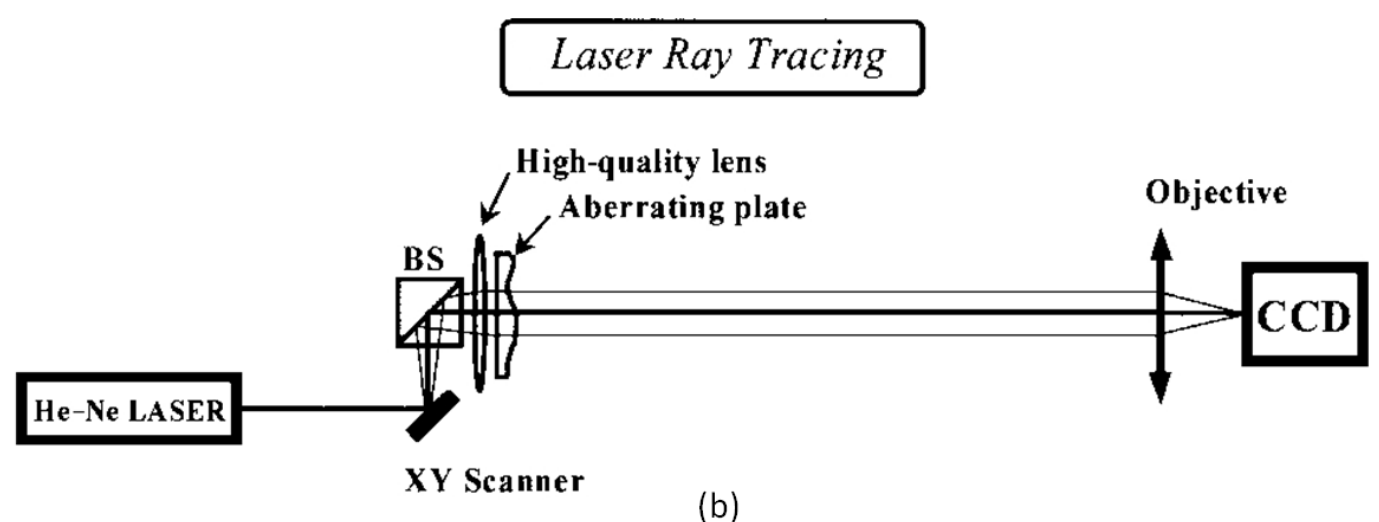

Figure 1.15 (a) Scheme of H-S. (b) Scheme of LRT (Navarro and Moreno-Barriuso 1999)

LRT is another method for measuring the path of the wavefront, which has many features in common with the $\mathrm{H}-\mathrm{S}$ method, allowing a rather close and direct comparison for reciprocal validation. The LRT is based on the light entering into the eye through different pupil positions across the dilated pupil to project a spot onto the retina and is an objective 'ingoing' aberrometry technique (Molebny 1997, Navarro and Losada 1997, MorenoBarriuso, Marcos et al. 2001, Marcos, Diaz-Santana et al. 2002). A CCD camera captures the aerial images as a function of the entry pupil conjugated to the retina. Rays entering the eye through eccentric locations get deviated from the central ray by the effect of aberrations. The local transverse aberration is measured as the angular distance between the centroid of each aerial image. The centroid of the image corresponds to a centered entry pupil and the wave aberration is estimated from the set of local transverse aberrations. A custom-developed LRT is the technique of choice to quantify the ocular aberrations in this thesis, and the actual laboratory implementation will be described in Section 2.2.1. 


\subsection{Customized eye modeling: linking geometry and aberrations}

\subsubsection{Phakic eye: Compensation between corneal and internal aberrations}

In order to understand the complex structure and optical properties of the Phakic eye, simplified optical models of real eyes with constructional parameters, generally means of population distributions, are used. These models vary in their complexity and thus suitability for applications. In 1662, Descartes exploited the first wide-angle optical eye model to understand the mapping of the visual hemisphere on the retinal surface. Then in 1652, Huygens developed the simplest of schematic eyes with one refracting surface at the front of eye. Schematic eye models with one spherical corneal and two lens surfaces were developed by Young (1801), Moser (1844), Listing (1851) and Tscherning (1898). Based on the improvement of the techniques for measuring the eye curvatures and axial distances, several eye models were proposed in the last century by Gullstrand (Gullstrand 1909) (shown in Figure 1.16) and Le Grand (Le Grand 1956). However, these simple models could not be used to fully understand the aberrations of each component. Then in 1997, Liou Brennan (Liou and Brennan 1997) provided a model for calculating optical transfer functions and predicting optical performance of the eye and Navarro reported a critical review of the optical design of the human eye in 2009(Navarro 2009).

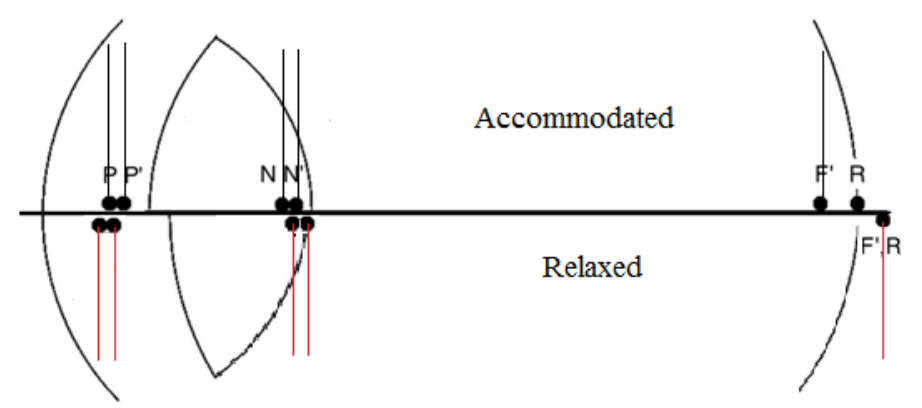

Figure 1.16 Schematic of Gullstrand-Emsley eye model (cardinal points at reasonable location; 10.9 $\mathrm{D}$ accommodated forms; anterior lens surface moved $0.4 \mathrm{~mm}$ with more curved anterior lens surface).

A good optical model eye (schematic eye) summarizes and organizes the understanding of the eye as an optical system, and it provides a conceptual framework for thinking about how the retinal image is formed to launch investigations into the visual process. In the three and four refracting surface models, the lack of a gradient index is compensated for by increasing the surface power via an equivalent index that is higher than occurs anywhere in the real lens.

Human eye models that incorporate a lens gradient index have been attempted since 1801, first by Thomas Young. The first eye model with lens structure was Gullstrand's extract eye with 2 corneal and 4 lens surfaces, with nested and homogeneous shells with different indexes on each surface. The refractive index of the outer cortex and inner nucleus were 1.386 and 1.406 . With the development of computers, ray tracing through gradient lens media has become commonplace, as demonstrated in Figure 1.17. 


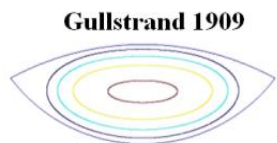

(a)

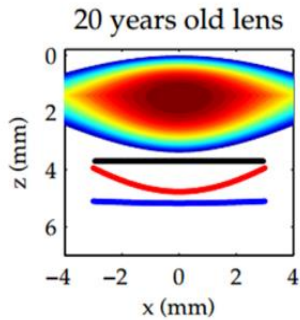

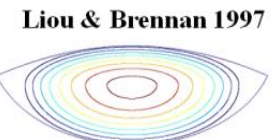

(b)

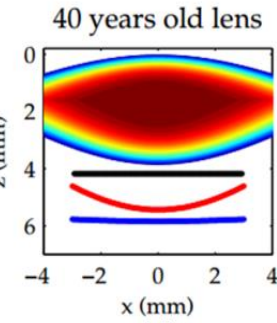

(d)

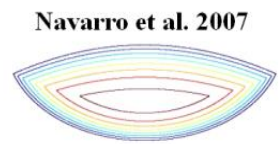

(c)

60 years old lens

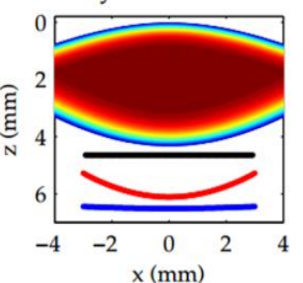

Figure 1.17 Models of gradient index with internal optical structure (a) Gullstrand 1909 (b) Liou \& Brennan 1997 (c) Navarro et al. 2007 (d) Alberto et al. 2010 (Crystalline lens GRIN with age function)

An increasing level of complexity occurs in optical model eyes as they better reflect the structure of real eyes, and the complexity of optical models will accelerate by refinement of measurement techniques and better technology. Among those surfaces and refractive media, the majority of all are aspherical surfaces, irregular surfaces or GRIN lenses, and average data of typical ocular dimensions have been used to construct schematic eyes. A summary of the features that the models are based on and the average population data used in each calculation is in Table 1.1

Table 1.1 Summary of the eye-models, number of surfaces in each model and average population data used in each model (MERINO 2015)

\begin{tabular}{|c|c|c|c|c|c|c|c|c|c|}
\hline \multirow{2}{*}{ Model(Year) } & \multirow{2}{*}{\multicolumn{2}{|c|}{$\begin{array}{c}\text { Surfaces } \\
\text { Spherical Aspheric }\end{array}$}} & \multirow{2}{*}{ Lens } & \multirow{2}{*}{ Accom. } & \multicolumn{2}{|c|}{ Domain } & \multirow{2}{*}{ Dispersion } & \multirow{2}{*}{ Age } & \multirow{2}{*}{ Ametropic } \\
\hline & & & & & On-axis & Off-axis & & & \\
\hline Huygens-1652 & & 1 & - & - & Yes & - & - & - & - \\
\hline $\begin{array}{c}\text { Young-1801 } \\
\text { Listing-1851 } \\
\text { Tscherning-1898 }\end{array}$ & 3 & - & - & Yes & Yes & - & - & - & - \\
\hline Gullstrand-1909 & 6 & - & Shell & Yes & Yes & - & - & - & - \\
\hline Le Grand-1956 & 4 & - & - & Yes & Yes & - & - & - & - \\
\hline Emsley-1952 & 1 & - & - & - & Yes & - & - & - & - \\
\hline Lotmar-1971 & 3 & 1 & - & - & Yes & Yes & - & - & - \\
\hline Drasdo \& Fowler-1974 & - & 4 & - & - & Yes & Yes & - & - & - \\
\hline Kooijman-1983 & - & 4 & GRIN & - & Yes & Yes & - & - & - \\
\hline Pomerantzeff et al. -1984 & - & 2 & Shell & - & Yes & Yes & - & - & - \\
\hline Navarro et al. -1985 & 1 & 3 & - & Yes & Yes & Yes & Yes & - & - \\
\hline Blaker-1991 & - & - & GRIN & Yes & Yes & - & - & Yes & - \\
\hline George Smith et al. -1992 & 2 & 2 & GRIN & - & Yes & - & - & Yes & - \\
\hline Indiana -1992 & - & 1 & - & - & Yes & Yes & Yes & - & - \\
\hline Liou \& Brennan -1997 & - & 2 & GRIN & - & Yes & Yes & Yes & - & - \\
\hline Norrby -2005 & - & 4 & - & Yes & Yes & Yes & - & Yes & - \\
\hline Atchison-2006 & - & 5 & GRIN & - & Yes & Yes & Yes & - & Yes \\
\hline Goncharov \& Dainty -2007 & - & 4 & GRIN & - & Yes & Yes & - & - & - \\
\hline Navarro -2007 & - & 5 & GRIN & - & Yes & Yes & Yes & - & Yes \\
\hline George Smith et al. -2009 & & 4 & GRIN & & Yes & Yes & - & Yes & - \\
\hline Campbell-2010 & - & 4 & Shell & Yes & Yes & Yes & - & Yes & - \\
\hline Rozema -2011 & - & 4 & - & - & Yes & - & - & - & - \\
\hline Juan Tabernero et al. -2011 & 1 (SAG) & 2 & - & Yes & Yes & Yes & Yes & Yes & Yes \\
\hline Ying-Ling Chen- 2012 & - & 4 & Shell & Yes & Yes & - & - & - & $\mathrm{KC}^{*}$ \\
\hline AshKan Arianpour et al. -2013 & 1 & 4 & - & - & Yes & Yes & - & - & - \\
\hline Polans-2015 & - & 4 & GRIN & Yes & Yes & Yes & Yes & Yes & - \\
\hline
\end{tabular}

* $\mathrm{KC}$ is keratoconus; 


\subsubsection{Pseudophakic eye: contributing factors to optical degradation in eyes implanted with IOLs}

Pseudophakic eyes are simpler than phakic eye models as the refractive index of the IOL is constant. The retinal image quality in a pseudophakic eye depends on the parameters of the implanted IOL, in particular on its chromatic aberration. Previous computer eye models use biometric information gathered from multiple devices (anterior cornea from Placido disk topography; tilt and decentration from Purkinje imaging apparatus; anterior chamber depth from slit lamp; axial length from low coherence interferometer), examples listed in Table 1.2. ZEMAX (Radiant ZEMAX; Focus software, Tucson, AZ), Code V (Optical Research Associates, Pasadena, CA) and OSLO (Lambda Research Corporation, Littleton, MA) are the most current ray tracing computational programs, which could assistant modeling and optical optimization by integrating a merit function in order to approach the specific targets (e.g. best focal position, optical quality metrics and automatic control parameters) by Matlab (Mathworks, Natik, MA). More detail about Zemax routines will be described in Section 2.4.1. More detail about Matlab routines will be described in Section 2.4.2.

There are several IOL designs and/or models available on the market: monofocal IOL (spherical, aspherical), multifocal IOL with an apodized diffractive design that optimally distributes light to distance, intermediate and near focal points, toric IOL that correct astigmatism and accommodative IOL (curvature changes of the lens surfaces and/or axial displacement). Aspheric IOLs are designed to correct spherical aberration and toric IOLs are designed to fully correct astigmatism. Premium monofocal IOLs design have an ability to accommodate like a Crystalline lens by a focusing process, which are called accommodative IOLs. However, several recent clinical evaluations of patients with accommodating IOLs (McLeod 2006, Ossma, Galvis et al. 2007, Sanders and Sanders 2007, Marcos, Ortiz et al. 2014) did not reveal the large axial shifts with either natural or drug-induced accommodation, as well as tilt changes with respect to natural lens and accommodative effort. OCT-based custom computer eye models in this thesis are also applicable to the optimization of the IOL power calculation, using virtual ray tracing to overcome many limitations of current IOL power selection techniques, and customized IOL designs. 
Biometric measurements in the crystalline lens: Applications in cataract surgery

Table 1.2 Summary of Pseudophakic eye model (MERINO 2015)

\begin{tabular}{|c|c|c|c|}
\hline \multirow[t]{2}{*}{ Model (Year) } & \multicolumn{2}{|c|}{ Cornea } & \multirow[t]{2}{*}{ IOL } \\
\hline & Anterior & Posterior & \\
\hline $\begin{array}{l}\text { Patricia A. Piers et al. } \\
\qquad-2004\end{array}$ & Placido-disk & - & Tecnis Z9000 (AMO); Phacof lex SI40NB (AMO) \\
\hline $\begin{array}{l}\text { Juan Tabernero et al. } \\
\qquad-2006\end{array}$ & Placido-disk & - & $\begin{array}{l}\text { Tecnis TM Z9000 (AMO); } \\
\text { the CeeOn TM } 911 \mathrm{~A}(\mathrm{AMO})\end{array}$ \\
\hline $\begin{array}{l}\text { Sverker Norrby et al. } \\
\quad-2006\end{array}$ & Placido-disk & - & $\begin{array}{l}\text { Theoretical; Tecnis Z9000 (AMO); } \\
\text { LI61U IOL, L161AO IOL (Bausch \& Lomb); } \\
\text { SofPort AO; SN60WF, SN60AT IOL (Alcon); KS- } \\
\text { 3Ai IOLs (Canon); the CeeOn TM 911A (AMO) }\end{array}$ \\
\hline Rosales et al. -2007 & Placido-disk & - & Aspheric IOLs (Acrysof IQ, Alcon); \\
\hline $\begin{array}{l}\text { Barbero \& Marcos } \\
\quad-2007\end{array}$ & Theoretical & Theoretical & Tecnis Z9000 (AMO); Theretical \\
\hline $\begin{array}{l}\text { Damian Siedlecki et al. } \\
\qquad-2007\end{array}$ & $\begin{array}{c}\text { Theoretical } \\
\text { (Liou-Brennan) }\end{array}$ & - & $\begin{array}{l}\text { P359UV (Bausch \& Lomb); AcrySoft MA60BM } \\
\text { (Alcon);AR40N (Allergan) }\end{array}$ \\
\hline $\begin{array}{l}\text { Jen Einighammer et al. } \\
\qquad-2009\end{array}$ & Placido-disk & - & $\begin{array}{c}\text { Theoretical models : Spherical IOL, Aspheric IOL, } \\
\text { Toric IOL and Toric aspheric IOL }\end{array}$ \\
\hline Canovas et al. -2011 & Placido-disk & - & Theoretical \\
\hline Zhu et al. -2011 & OCT & OCT & Theoretical \\
\hline Ribeiro et al. -2012 & ORBSCAN & ORBSCAN & Theoretical \\
\hline A. Drauschke et al. -2012 & $\begin{array}{c}\text { Theoretical } \\
\text { (Liou-Brennan) }\end{array}$ & - & Theoretical \\
\hline $\begin{array}{l}\text { AshKan Arianpour et al. } \\
\qquad-2013\end{array}$ & Theoretical & Theoretical & Theoretical \\
\hline Fernández et al. -2013 & Theoretical & Theoretical & $\begin{array}{l}\text { AcrySof ReSTOR SN60D3, SN6AD3, SN6AD1 } \\
\text { (Alcon); Acri.LISA 366D }\end{array}$ \\
\hline Zhang et al. -2015 & $\begin{array}{c}\text { Theoretical } \\
\text { (Hwey-Lan Liou) }\end{array}$ & $\begin{array}{c}\text { Theoretical } \\
\text { (Hwey-Lan Liou) }\end{array}$ & AcrySof Toric SN60T5 IOL, SN60AT IOL (ALcon); \\
\hline
\end{tabular}




\subsection{Goals of this thesis and hypothesis}

\subsubsection{Goals of this Thesis}

- To study the intraocular lens alignment from an en-face OCT imaging Purkinje-like method. Measurement of implanted IOL alignment in cataract surgery patients is important for understanding their optical performance. This thesis presents a method to estimate misalignment (tilt and decentration) of IOLs based on en-face OCT images showing Purkinje-like images that correspond to the specular reflections from the corneal and IOL surfaces. Unlike in standard Purkinje imaging, the tomographic nature of OCT allows unequivocal association of the reflection with the corresponding surface. The methodology was demonstrated on an artificial model eye and pseudophakic eyes.

- To analyze Crystalline lens topography in vitro eyes of different ages and to understand how OCT 3D surface topography of isolated human crystalline lenses changes with age. This thesis presents radii of curvature, astigmatism and highorder irregularities of the crystalline lens' anterior and posterior lens surfaces. Understanding the coordination of anterior and posterior lens surface geometries and their topographical changes with age sheds light on the role of the lens in the optical properties of the eye and the lens aging mechanism.

- To simulate ocular aberrations on an individual level from full 3D OCT based pseudophakic custom computer eye models and validate with experimental ocular aberrations from LRT. This thesis shows that customized eye models in combination with the actual measurements of ocular aberrations can be used to identify the relative importance of each factor. The measurements of ocular aberrations in pseudophakic eyes allow assessment of the overall optical performance and show the capability of building computer eye models from information all obtained simultaneously with the one instrument.

- To analyze the effect on optical quality of IOL decentration and to identify optimal IOL centration using OCT-based ray tracing on pseudophakic eyes. Quantitative OCT proves an excellent technique for pre-operative 3D biometry and post-cataract surgery evaluation. This thesis presents the evaluation of the contributing factors to optical degradation in eyes implanted with IOLs and the impact of IOL misalignment in particular. This thesis will be useful to investigate the potential for OCT-based model eyes, to become the reference for ray tracing based IOL premium centration selection (pre-operatively) and to understand mechanisms of operation and functional outcomes of premium IOLs. 


\subsubsection{Hypothesis}

Our hypothesis is that knowledge of the IOL position will help us to better understand the optical quality of the pseudophakic eye by measuring IOL tilt and decentration after cataract surgery (with OCT based Purkinje-like imaging methods implemented and validated in this thesis). Quantitative measurements of the crystalline lens based on Optical Coherence Tomography will allow us to understand the sources of ocular aberrations. With the aid of full OCT based customized computer eye models, we will be able to predict the role of each factor to optical quality on individual leaver, and these advances will contribute to customize IOL power calculation and improvement of cataract surgery. 


\subsection{Structure of this thesis}

The body of this thesis is structured as follows:

Chapter 1 presents the background, motivation, goals and hypothesis of the thesis.

Chapter 2 describes the methods used throughout this thesis, including the SD-OCT (for geometrical and aberration measurements of the anterior segment of the eye), the Laser Ray Tracing (for ocular aberration measurements), other techniques for validation, the optical quality metrics, ex vivo and in vivo measurements, and virtual ray tracing calculations.

Chapter 3 presents a method to estimate tilt and decentration of IOLs based on OCT images. En-face OCT images show Purkinje-like images that correspond to the specular reflections from the corneal and IOL surfaces. The locations of the Purkinje-like images are linear combinations of IOL tilt, IOL decentration, and eye rotation. The weighting coefficients depend on the individual anterior segment geometry, obtained from the same OCT datasets. The methodology was demonstrated on an artificial model eye with set amounts of lens tilt and decentration, and then on five pseudophakic eyes. Purkinje-based methodology from anterior segment en-face OCT imaging will provide reliable measurements of IOL tilt and decentration.

Chapter 4 uses quantitative 3D Optical Coherence Tomography to measure the surface topography of 36 isolated human lenses and to evaluate the relationship between anterior and posterior lens surface shape and their changes with age. The anterior surface RMS spherical term, RMS coma and $3^{\text {rd }}$ order RMS decrease significantly with age. In general, there is a statistically significant correlation between the $3^{\text {rd }}$ and $4^{\text {th }}$ order terms of the anterior and posterior surfaces. Understanding the coordination of anterior and posterior lens surface geometries and their topographical changes with age sheds light on the role of the lens in the optical properties of the eye and the lens aging mechanism.

Chapter 5 compares measured wave aberrations in pseudophakic eyes implanted with aspheric IOLs with simulated aberrations from numerical ray tracing on customized computer eye models. This study shows that full OCT-based pseudophakic custom computer eye models allow for understanding the relative contribution of optical geometrical and surgically-related factors to image quality, and are an excellent tool for characterizing and improving cataract surgery.

Chapter 6 validates custom computer eye models built using quantitative all OCT-based geometry to identify the intraocular lens (IOL) centration that would produce the best optical quality using Visual Strehl (VS). OCT-based customized eye models accurately predict optical quality in pseudophakic eyes implanted with state-of-the-art monofocal IOLs. Zero IOL decentration does not produce optical quality. The best IOL decentration can be obtained for each patient based on personalized models, and the achieved improvement may be of clinical relevance.

Chapter 7 and 8 enumerate the conclusions of the thesis, future work and publications written and activities performed during this $\mathrm{PhD}$ period. 


\section{CHAPTER 2}

\section{EXPERIMENTAL METHODS,}

\section{COMPUTATIONAL}

TECHNIQUES AND OTHER

TYPES OF VALIDATION 


\section{RESUMEN}

Este capítulo describe métodos experimentales, técnicas computacionales y otros tipos de validación utilizados en este trabajo.

Este capítulo describe un nuevo desarrollo específico para estimar la inclinación y descentración de la lente cristalina o lentes intraoculares. El método está inspirado en los métodos de imagen de Purkinje implementados previamente en VioBio Lab, pero aprovecha la naturaleza transversal de OCT que permite identificar de manera inequívoca las reflexiones de cada superficie. El algoritmo personalizado de procesamiento de imágenes escrito segmenta el borde de la pupila, encuentra el centro por ajuste de elipse y las posiciones de las imágenes de tipo Purkinje, PI, PIII y PIV a partir de las imágenes OCT en-face. El desalineamiento de la lente y la fovea se obtienen asumiendo relaciones líneales entre aquéllos y las posiciones de las imágenes de Purkinje. El capítulo describe también el sistema de OCT de desarrollo propio utilizado para la cuantificación de la geometría del cristalino del ojo ex vivo y del segmento anterior en pacuentes pseudofáquicos in vivo.

Los datos de elevación basados en OCT de las superficies corneales anteriores y posteriores, las distancias del segmento anterior, la geometría de la LIO, la alineación de la LIO, el ángulo de rotación del ojo lambda $\lambda$ y la longitud axial mediante interferometría de baja coherencia fueron exportados a un sistema de diseño óptico (Zemax) donde se simularon modelos computacionales de ojos pseudofáquicos. Estos modelos se utilizadon para la estimación de coeficientes de ponderación en el método de Purkinje, la medida de la inclinación y descentración, cálculos de aberraciones de alto orden y cálculo de la posición lateral óptima de la IOL.

Otros métodos experimentales usados en la tesis y descritos en el capítulo incluyen la profilometría de no contacto basada en microscopía confocal (sistema comercial de Sensorfar) y el Trazado de Rayos por Laser previamente desarrollado en el laboratorio para la medida de las aberraciones del ojo 
Experimental methods, computational techniques and other types of validation

\subsection{D-Anterior segment spectral domain OCT}

\subsubsection{Custom setup}

In a spectral domain OCT (SD-OCT) system, a spectral fringe pattern (channeled spectrum) is recorded by a spectrometer with a line scan camera, and a tomogram line is calculated by unevenly sampled Fourier transformation of the recorded fringe pattern. This technique can perform imaging much faster without mechanical depth scanning and allow the collection of 3D anterior segment data in hundreds of milliseconds with a high lateral and axial resolution to evaluate ocular anterior segment biometry (from anterior cornea to the posterior crystalline lens/IOL).

The customized SD-OCT system (Grulkowski, Gora et al. 2009) in the VioBio lab was developed in collaboration with Nicolaus Copernicus University (Torun, Poland) (Figures 2.1 and 2.2 show a picture and the schematic layout of the system) and was provided in the last years with several additional elements (Gambra, Ortiz et al. 2013) and customdeveloped processing routines (Ortiz, Siedlecki et al. 2010). The SD-OCT system includes the main components (shown in Figure 2.2: light source; reference arm; sample arm (including accommodation/fixation channel, scanning galvanic mirror, objective lens and subjects); spectrometer (including condensing lens, grating, and line-scan camera).

The light source consists of a superluminescent diode (SLD) $(\lambda 0=840 \mathrm{~nm}$, near Gaussian emission bandwidth 50nm, Superlum, Ireland). The Laser beam is split into reference and sample arms by a fiber mate to an 80:20 fiber coupler (FC) in order to avoid back reflected light from the reference and sample arms returning to the SLD. The reference arm consists of a collimating lens to produce a collimated beam with a polarization controller to optimize detection performance, a neutral density filter to increase or decrease the power of light in the reference arm, a converging lens and a reference mirror.

The additional beam splitter is placed in the sample arm which incorporates the fixation channel for measuring purposes. In this channel, a Badal system composed of two achromatic doublets of equal focal lengths of $150 \mathrm{~mm}$, that form an afocal system of magnification $\mathrm{x} 1$, and two flat mirrors, mounted on a motorized stage (VXM-1, Velmex) which can be moved to change the optical path between the lenses. That allows compensating spherical refractive errors. Vergence is changed with the Badal system from $-10 \mathrm{D}$ to $10 \mathrm{D}$ and compensate with trial lenses placed in the pupil plane. In all conditions, the image remains in focus (in 1-D steps). For the fixation channel, the fixation stimulus consists of a 20/25 white Snellen E-letter presented on a black background on a DigitalLight-Processing (DLP) picoprojector (854x480 pixels, Picopix PPX2055/F7, Philips NV, Amsterdam, Netherlands; 55 Lumens) subtending a 5-arcmin visual angle. Two neutral density filters (ND 16) are placed after the picoprojector to produce an average luminance of $\sim 30 \mathrm{~cd} / \mathrm{m}^{2}$ in an otherwise dark environment.

To perform the measurements in vivo, the target is first aligned with the OCT axis and then the target is moved along the horizontal and vertical meridians for measuring in the line of sight using a script written in Matlab. The collimating lens produces a collimated beam on the cornea. The horizontal and vertical scanning is produced by the galvanometer optical 
scanners (Cambridge Technology Inc., Bedford, Massachusetts), which are driven by an analog input/output card (National Instruments, Austin, Texas), and an objective lens to collimate the chief rays of the beams and to focus the irradiance impinging the sample. The light reflected back by the reference and sample arm is recombined by the beam splitter and it is led to the spectrometer.

A spectrometer consists of a condensing lens to collimate the light, a volume diffraction grating to spatially separate the spectrum and a magnifier objective compose of two converging lenses to focus the signal on the detector, a 12-bit line-scan CMOS camera with 4096 pixels (Basler sprint spL4096-140k; Basler AG, Germany), is used to measure the interference fringes of the spectrum.

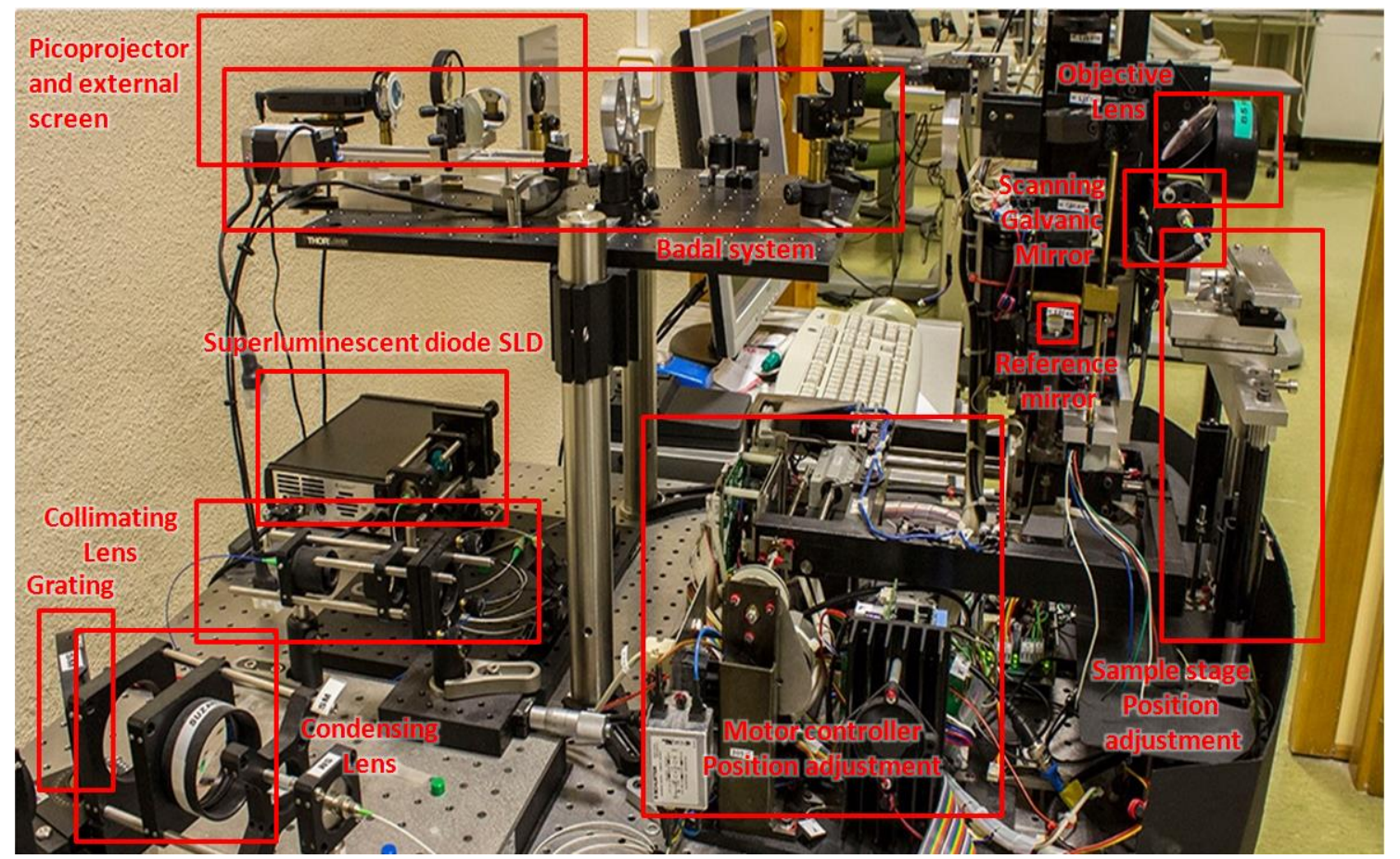

Figure 2.1 Photograph of the actual custom Spectral-Domain Optical Coherence Tomography device at the VioBio lab (Instituto de Óptica, "Daza de Valdés") 
Chapter 2

Experimental methods, computational techniques and other types of validation

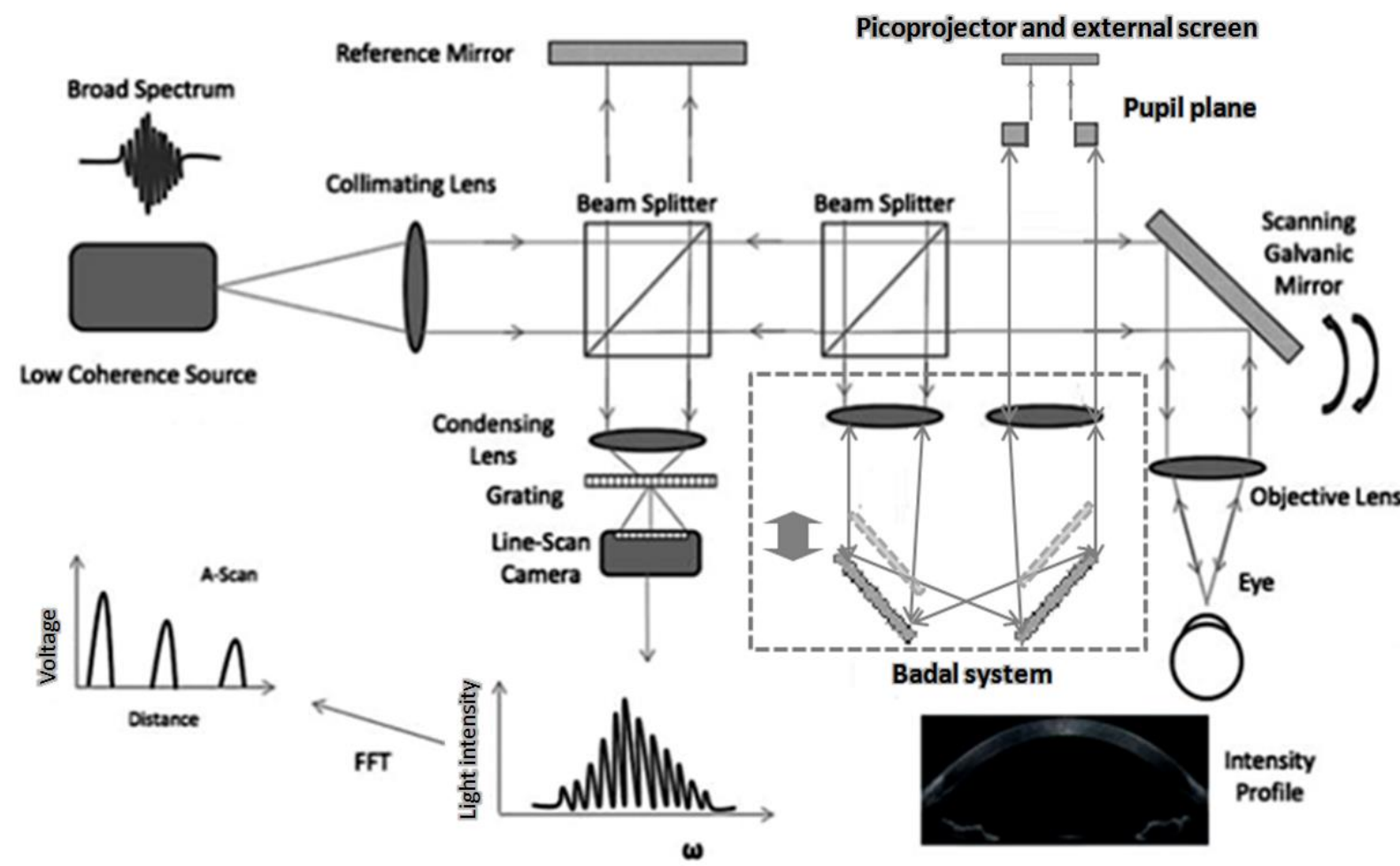

Figure 2.2 The schematic diagram of the custom SD-OCT set-up, components: Low coherence broad band spectrum source; Collimating lens; Beam Splitter 1; Reference mirror; Beam splitter 2; Badal system; Picoprojector and external screen; Scanning galvanic mirror; Objective lens; Condensing lens; Grating; Line-scan CMOS camera 


\subsubsection{Quantification tools}

When imaging even perfectly flat surfaces by means of 3D OCT devices, they become curved due to fan distortion (shown in Figure 2.3). This distortion can be described as a combination of at least two possible effects: (1) architecture of the scanning system, with relatively significant influence by the spatial separation of the mirrors; (2) design, position and alignment of the collimation lens in relation to the mirrors of the scanner. Furthermore, optical distortion is an effect produced while imaging a sample embedded in a medium different from vacuum (air), and therefore the rays are bended by the effect of refraction from preceding surfaces. Apart from the strongest refractive effect at the air-anterior cornea (or tear film) interface, the major effects of refraction in a normal eye will happen in the posterior cornea-aqueous humor, aqueous humor-anterior lens, and posterior lens-vitreous humor interfaces.

Due to these distortions, OCT images need to be corrected for quantification. Customalgorithms correct the OCT data from fan distortion (arising from the scanning architecture) and optical distortion (due to the refraction in the optical surfaces) (Ortiz, Siedlecki et al. 2009, Ortiz, Siedlecki et al. 2009, Ortiz, Siedlecki et al. 2010, Ortiz, Siedlecki et al. 2011, Ortiz, Perez-Merino et al. 2012, Ortiz, Perez-Merino et al. 2013, Perez-Merino, VelascoOcana et al. 2015).

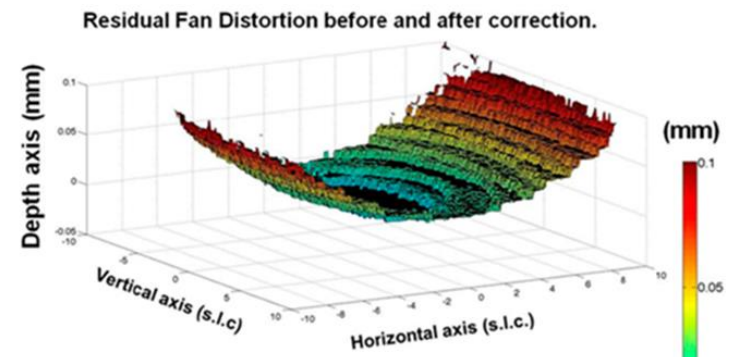

(a)

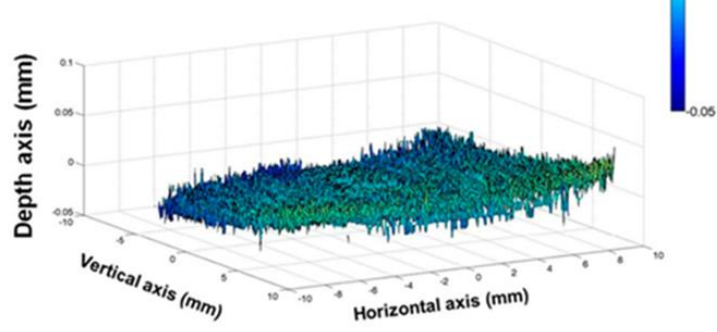

(b)

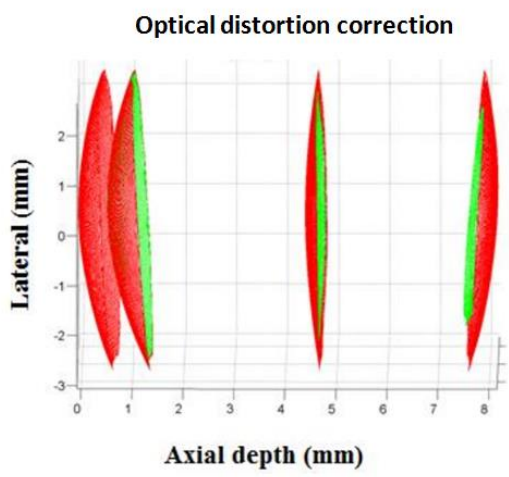

(c)

Figure 2.3 Map of residual fan distortion before (a) and after (b) correction, obtained from a flat optical surface with the OCT system of the study. Reproduced from (Ortiz, Siedlecki et al. 2010, Ortiz, Siedlecki et al. 2011); (c) Illustration of the effect of the 3D distortion correction on the anterior segment surfaces (all surfaces are corrected axially from refractive index. Green: corrected volumes; red: uncorrected volumes). Reproduced from (Ortiz, Siedlecki et al. 2009, Perez-Merino, Velasco-Ocana et al. 2015).

Dedicated image processing routines allow the segmentation of the optical surfaces and quantification of their geometry (Ortiz, Siedlecki et al. 2009, Ortiz, Siedlecki et al. 2009, Ortiz, Siedlecki et al. 2010, Ortiz, Siedlecki et al. 2011, Ortiz, Perez-Merino et al. 2012, Ortiz, Perez-Merino et al. 2012, Ortiz, Perez-Merino et al. 2013). Custom image processing 
Experimental methods, computational techniques and other types of validation

algorithms for geometrical characterization of the OCT images, described in detail in the previous studies of our lab (Ortiz, Siedlecki et al. 2009, Ortiz, Siedlecki et al. 2009, Ortiz, Siedlecki et al. 2010, Ortiz, Siedlecki et al. 2011, Ortiz, Perez-Merino et al. 2012, Ortiz, Perez-Merino et al. 2012, Ortiz, Perez-Merino et al. 2013) are comprised of: image denoising, statistical thresholding, volume clustering, multilayer segmentation, further surface denoising, pupil centration, 3D merging and surface fitting by sphere/conicoids and elevation/topographic maps (Zernike polynomial). Custom-developed image processing algorithms are applied for automatic structure detection (more detail will be described in Section 2.6.2).

The large amount of noise appearing in the OCT images produces low contrast data, therefore improving the detection ratio by image denoising is necessary for quantification. In order to reduce the noise and to enhance the contrast, the denoising filter is applied by averaging the pixel information across multiple scales and orientations.

After denoising the OCT images, the signal is separated from the background, taking into account the existing difference in statistics of the signal and the background. The algorithm of statistical thresholding classifies every single pixel of the data by its maximum likelihood of belonging to a certain class, and only those points belonging to classes different from noise are considered. As a result of the overlapping statistics of the noise and signal, randomly distributed noise is still present in the images after thresholding.

A volume clustering algorithm based on area or volume classification was developed, which allows identifying a layer if there was continuity in at least one of the directions of the tensor mask (which is 3D convolved with the tensor of data).

Based on the first derivative boundary region identification, an algorithm extracts the position of the peaks of every A-scan and sorts those peaks by position and intensity for multilayer segmentation with the classified volumes.

Even after the denoising algorithm and boundary detection algorithms, the segmented surfaces are still noisy due to the OCT system adding noise during the detection process. The noise can be described as a ripple in the smooth surface of the anterior segment of the eye based on a Zernike description of the surface (more detail will be described later in the surface fitting paragraph). The Zernike expansion is uniformly sampled over the area of interest and then the finite-element ray-tracing algorithm is applied and used to obtain smooth surfaces from noisy raw elevation maps, acting as a sort of low pass filter for further surface denoising.

The pupil center is used as a reference across measurements (i.e. cornea, anterior and posterior lens) to define the optical zone (effective area within the pupil, shown in Figure 2.4). The corneal image is inverted, since for efficiency in the OCT focus range it is obtained on the opposite side of the Fourier transform.

Then, the merging 3D volumes of cornea/iris/anterior and iris/anterior/posterior lens are merged by the pupil center reference with help of the specular reflections and rotated to superimpose the characteristic vectors of the corresponding pupil plane. The pupil centration could also be used as a reference for pre- and post-operative analysis of anterior 
segments. Therefore, the pupil coordinates in 3D along with the normal to the pupil plane are used for merging. The specular reflections from corneal and lens/IOL surfaces are also used as a fixed reference for further merging 3D volumes. Furthermore, the IOL tilt and decentration could be calculated by those specular reflections based on Purkinje methodology, which will be described in Section 2.1.3.

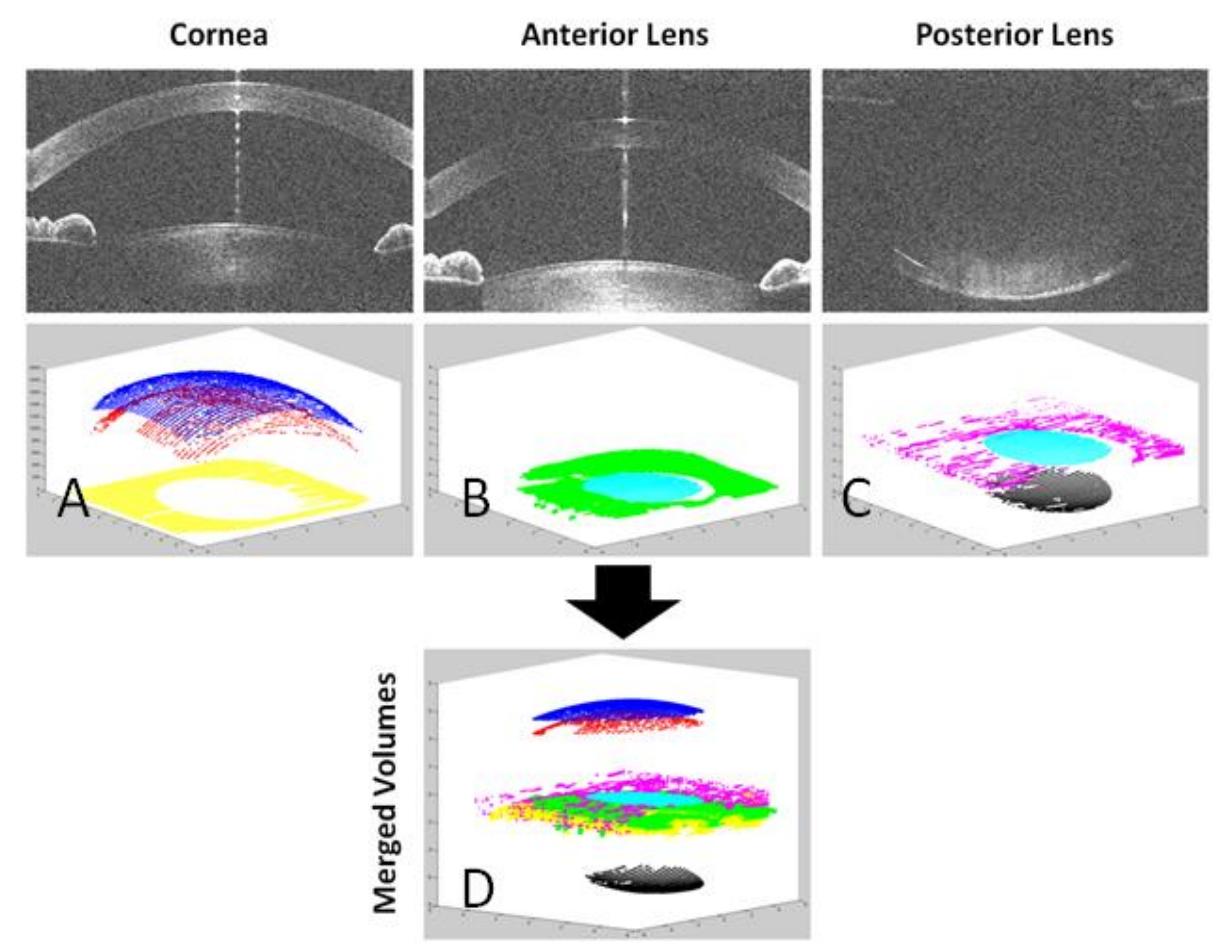

Figure 2.4 Illustration of pupil centration as reference across: A: anterior and posterior cornea, iris; B: iris and anterior lens; C: pupil, anterior lens and posterior lens in effective area within the pupil; D: merged volumes of anterior segments. Blue and red points present anterior and posterior cornea surfaces respectively; Light blue and black points present anterior and posterior lens surfaces respectively; Yellow, green and pink points present iris.

Once the surfaces were corrected for fan distortion and optical distortion as stated before, they could be fit by standard functions: spherical, conicoidal and topographic/elevation maps (Zernike polynomials). The same custom routines for surfaces fitting are also used to fit the corneal elevation maps provided by other instruments (Scheimpflug) used for reference (more detail will be described in Section 2.3.1), and crystalline lens topographies.

\section{Sphere:}

$\left(\mathrm{x}-\mathrm{x}_{0}\right)^{2}+\left(\mathrm{y}-\mathrm{y}_{0}\right)^{2}+\left(\mathrm{z}-\mathrm{z}_{0}\right)^{2}=\mathrm{a}^{2}$

Where surface data is $\left(\mathrm{x}_{0}, \mathrm{y}_{0}, \mathrm{z}_{0}\right)$, and $\mathrm{a}$ is the radius of sphere surface.

\section{Conicoids:}

$\left(\mathrm{x}-\mathrm{x}_{0}\right)^{2}+\left(\mathrm{y}-\mathrm{y}_{0}\right)^{2}-2\left(\mathrm{z}-\mathrm{z}_{0}\right) \mathrm{R}+(\mathrm{Q}+1)\left(\mathrm{z}-\mathrm{z}_{0}\right)^{2}=0$

Where the surface data is $\left(\mathrm{x}_{0}, \mathrm{y}_{0}, \mathrm{z}_{0}\right), \mathrm{R}$ is the radius of the conicoids surface and $\mathrm{Q}$ is the conic constant. 


\section{Topographic/elevation maps (Zernike polynomials):}

Free-form surfaces, such as irregular ocular surfaces in the human eye, cannot be described with simple surfaces. We use a Zernike polynomial representation of the surfaces following subtraction of the best fitting sphere, as described in earlier studies by multiple authors (Schwiegerling, Greivenkamp et al. 1995, Barbero, Marcos et al. 2002, Sicam, Coppens et al. 2004, Turuwhenua 2007). Zernike polynomials are a set of orthogonal polynomials that describe a wavefront for optical systems or description of any arbitrary surface shape over the unit circle (Svechnikov, Chkhalo et al. 2015).

There are even $Z_{n}^{m}$ and odd $Z_{n}^{-m}$ Zernike polynomials in Figure 2.5. The even polynomials are defined as $Z_{n}^{m}(\rho, \varphi)=\mathrm{R}_{n}^{m}(\rho) \cos (\mathrm{m} \varphi)$ and the odd polynomials as $Z_{n}^{-m}(\rho, \varphi)=\mathrm{R}_{n}^{m}(\rho) \sin (\mathrm{m} \varphi)$, where $\mathrm{m}$ and $\mathrm{n}$ are nonnegative integers with $\mathrm{n} \geq \mathrm{m}, \quad \varphi_{\text {is }}$ the azimuthal angle and $\rho$ is the radii distance $0 \leq \rho \leq 1$. The radial polynomials $R_{n}^{m}$ are defined as

$$
R_{n}^{m}(\rho)=\sum_{k=0}^{\frac{n-m}{2}} \frac{(-1)^{k}(\mathrm{n}-k) !}{k !\left(\frac{n+m}{2}-k\right) !\left(\frac{n-m}{2}-k\right) !} \rho^{n-2 k}
$$

Each polynomial describes a specific form of surface deviation. Their combined sum can produce a large number of more complex surface shapes and can be used to obtain smooth surfaces from noisy raw elevation maps, acting as a sort of low pass filter for noise. In principle, by including a sufficient number of Zernike polynomials (commonly referred to as terms), any topography map can be described to a desired degree of accuracy. Anterior and posterior cornea, anterior and posterior crystalline lens and IOL elevation maps were fit to a sixth-order polynomial expansion (with 28 terms), thus obtaining quantitative information of the ocular anterior segment (corneal topography, thickness and radii; anterior chamber depth; lens/IOL geometry, thickness, tilt and decentration). 


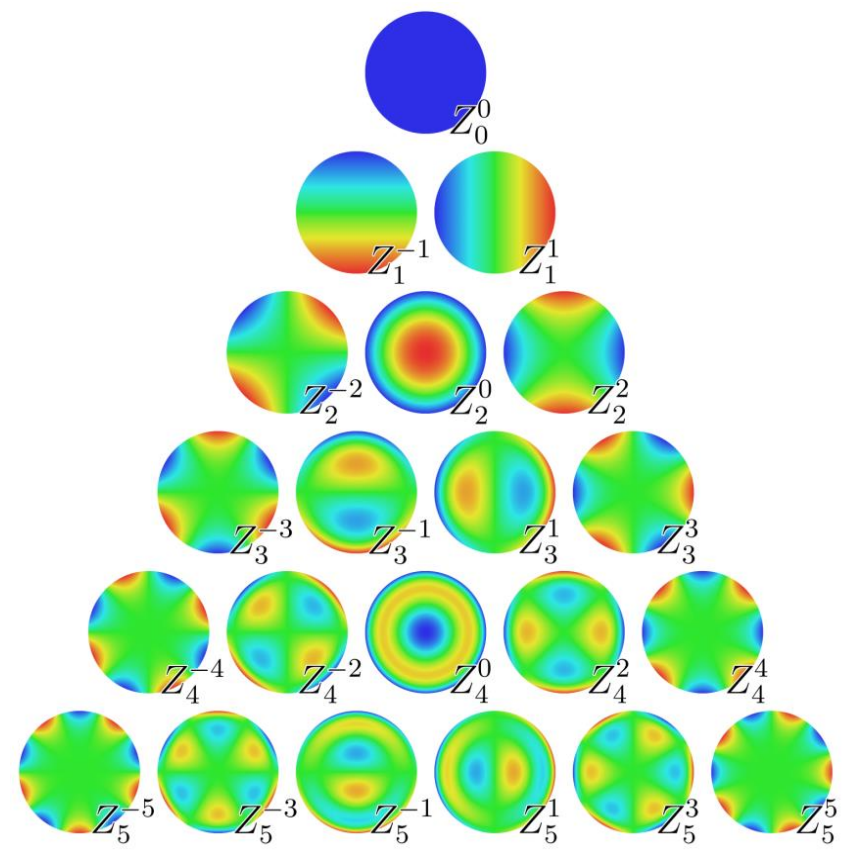

Figure 2.5 Coefficients of the Zernike polynomials up to the 6th radial order with 28 terms using the Optical Society of America (OSA) standard (Thibos, Applegate et al. 2002)

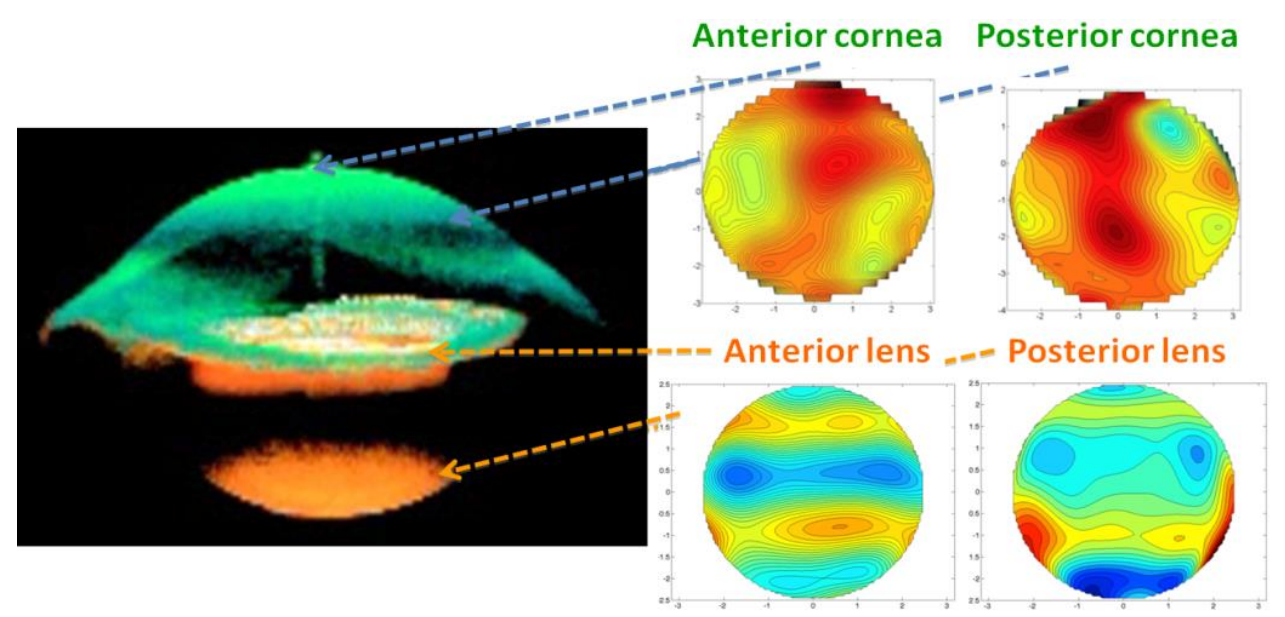

Figure 2.6 Quantitative anterior and posterior cornea and crystalline lens topography, after full distortion correction. Maps are Zernike fits to the elevation maps, relative to the best fitting sphere. (Ortiz, Perez-Merino et al. 2012) 


\subsubsection{Purkinje-like OCT en-face reflection image to measure lens tilt and decentration}

A specific new development of this thesis has been a method to estimate the tilt and decentration of the crystalline lens or intraocular lenses. The method is inspired by the Purkinje imaging methods previously implemented at VioBio Lab (Rosales, Dubbelman et al. 2006, Rosales and Marcos 2006, de Castro, Rosales et al. 2007, Rosales and Marcos 2007, Rosales, Wendt et al. 2008, Rosales and Marcos 2009), but takes advantage of the cross-sectional nature of OCT which allows unmistakengly identifying reflections from each surface. Purkinje images are reflections of the light from anterior and posterior corneal surfaces (first and second Purkinje images, PI, PII) and from anterior and posterior crystalline/intraocular lens surfaces (third and fourth Purkinje images, PIII, PIV). OCT technology resembles the Purkinje method, since three spots are observed in the en-face OCT images in Figure 2.8 (a). In contrast to the standard Purkinje method, the spots are not formed by the entire area of the ocular surfaces. Instead, the OCT system collects the scattered light reflected back from the tissue, and when the reflected ray direction coincides with the OCT axis, a strong specular reflection is captured in the test arm of the OCT, producing a bright spot in the en-face OCT images. Unlike in the standard Purkinje method, which relies on the images in a single plane of focus, the tomographic information of the OCT images allows one to relate each reflection with the corresponding surface, as well as avoiding image overlapping. The Purkinje image analysis (estimation of coefficients in a set of linear equations) requires assumption of an eye model. The assumption of a general computer eye model results in a decrease in the accuracy of the tilt and decentration estimates (Rosales and Marcos 2006). Instead, individual anatomical and biometrical parameters are used, generally obtained from corneal topography or keratometry, optical biometry, and Purkinje phakometry. These parameters can be retrieved from the same OCT dataset (Ortiz, Perez-Merino et al. 2012, Ortiz, Perez-Merino et al. 2013).

The Purkinje-like analysis can be summarized in five steps:

- Collection of a volumetric dataset

- Generation of image intensity values in the axial direction [Figure 2.7(a)]

- Pupil segmentation and identification of the positions of the specular reflexes on the different surfaces (Purkinje-like images positions, PI, PIII, and PIV) [Figure 2.7(b)]

- Estimation of weighting coefficients A-G that depend on the individual anterior segment geometry

- Calculation of eye rotation, lens tilt and decentration from Purkinje Formulas. The surfaces PI, PIII, and PIV are easily identified by checking the corresponding OCT B-Scan image [Figure 2.7(c)]. 


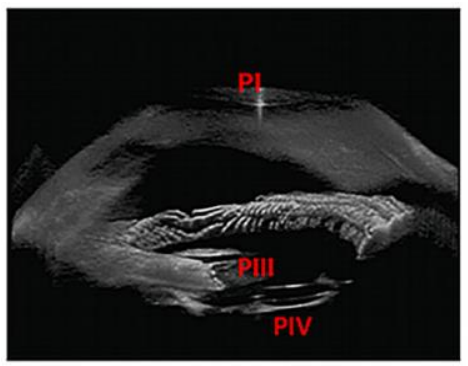

(a)

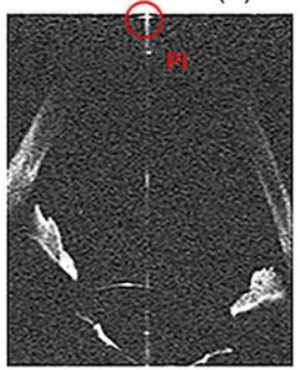

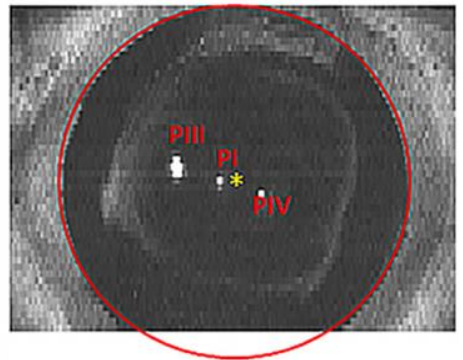

(b)

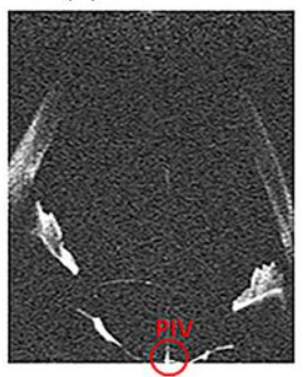

(c)

Figure 2.7 (a) Three-dimensional (3D) optical coherence tomography (OCT) image; (b) en-face OCT image showing the pupil merging, the locations of PI, PIII, and PIV in red and pupil center in yellow; (c) PI (left), PIII (center), and PIV (right) in OCT B-Scan images.

To resolve the global coordinates of 3D OCT images, we placed a USAF 1951 as a target, shown in Figure 2.8 (a), to scan by SD-OCT in our lab. The fundus view from the customized SOCT Data Processing program, developed previously in VioBio, is mirror symmetric to the target, shown in Figure 2.8 (b). The fundus OCT image is saved as a JPG image automatically with the proportion of A- and B-scans from the SOCT Data Processing program and batched B-scan from left to right with increasing image numbers, but it is rotated 90 degrees counterclockwise, shown in Figure 2.8 (c). For the collection of a volumetric dataset, a customized Matlab algorithm is developed to batch B-scan input to represent OCT fundus images automatically as saved, to restore image authenticity by rescaling the image from the proportion of A-and B-scans OCT image (300/50) setting to be the size of measurement $(15 / 7 \mathrm{~mm})$ shown in Figure $2.8(\mathrm{e})$, to rotate saved fundus images 90 degrees clockwise as shown in Figure 2.8 (f), and to flip the horizontal axis, due to the mirror symmetric scanning, to have real en-face OCT images, shown in Figure 2.8 (g).

The tilt and decentration nomenclature and sign conventions are illustrated in Figure 2.7(c). IOL tilt is defined as the angle between the IOL axis and the pupillary axis, where the IOL axis is the line joining the centers of curvature of the IOL and the pupillary axis joining the center of curvature of the cornea and the pupil center. IOL decentration is defined as the distance between the IOL center and the pupil center. According to the existing conventions (Rosales and Marcos 2006), positive horizontal decentration stands for nasal decentration (in right eyes) and temporal decentration (in left eyes), and vice versa for negative; and positive vertical decentration for superior, and negative for inferior. Positive tilt around the horizontal axis (tilt $\mathrm{x}$ ) indicates that the superior edge of the lens is moved forward. Negative tilt around the vertical axis (tilt y), in right eyes, indicates that the temporal edge of the lens is moved forward, and, in left eyes, indicates that the nasal edge of the lens is moved forward. 


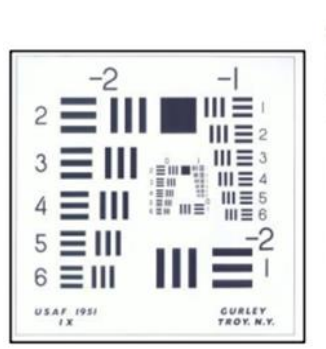

(a)

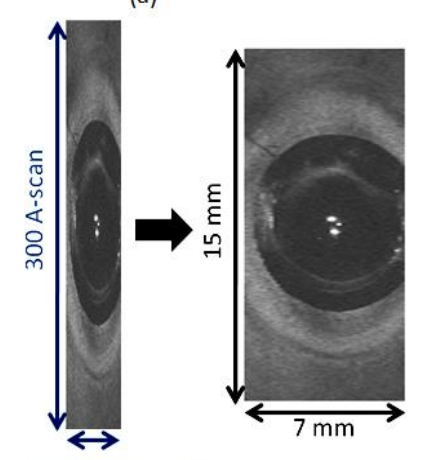

(d)

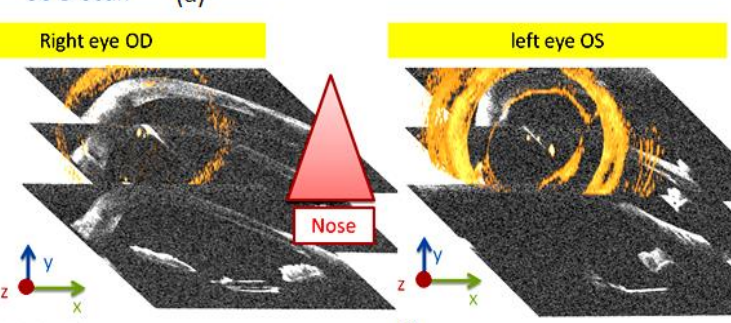

(f)

(b)
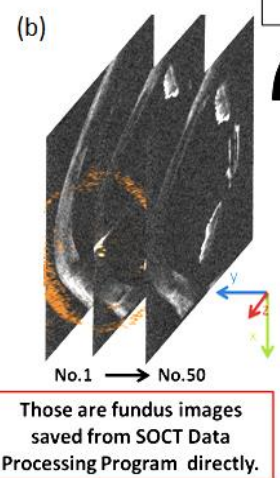
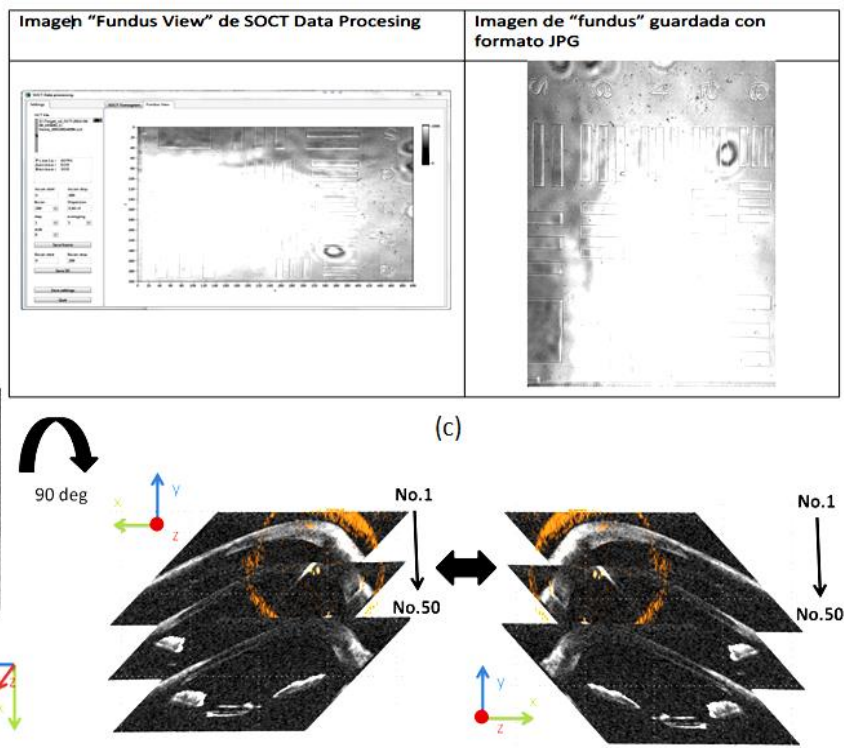

Processing Program directly.

present fundus image in SOCT Data Processing Program (e)

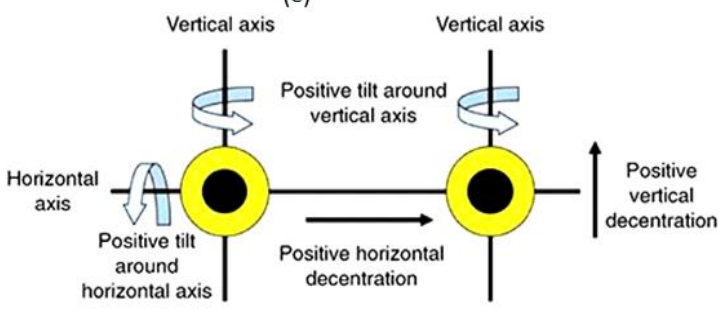

(g)

Figure 2.8 (a) USAF 1951; (b) OCT fundus image in SOCT data process program ; (c) OCT fundus image rotated 90 deg counterclockwise saved as JPG image; (d) Rescaling the height and width of JPG from $300 \mathrm{~A}$-scans and 50 B-scans OCT image as taken during OCT measuremnts15x7 mm measurement setting; (e) Image fundus rotation 90 degree clockwise and flipping around horizontal axis to have real en-face OCT image;(f) The definition of global coordinates of eye rotation, IOL tilt and decentration based on en-face OCT image; ( $\mathrm{g}$ ) Nomenclature and sign conventions for eye rotation, IOL tilt and decentration looking into the human eyes, as seen by the observer. The coordinate is the same as (Rosales and Marcos 2006).

The custom algorithm of image processing written in Matlab (Mathworks Inc., Natick, MA) segments the pupil edge and finds the center by ellipse fitting. Their Purkinje-like image positions, PI, PIII, and PIV are manually identified horizontal and vertical coordinates relative to the center of the pupil, are saved automatically. The method for estimating IOL alignment is based on that described in previous works (Phillips, Perez-Emmanuelli et al. 1988, Barry, Dunne et al. 2001, Rosales and Marcos 2006). This method assumes a linear relationship between Purkinje-like image positions (PI, PIII, PIV) and rotation of the eye $(\beta)$, IOL tilt $(\alpha)$, and decentration (d):

$$
\begin{aligned}
& P I=E \times \beta \\
& P I I I=F \times \beta+A \times \alpha+C \times d \\
& P I V=G \times \beta+B \times \alpha+D \times d
\end{aligned}
$$


A computer eye model was created in ZEMAX (Radiant ZEMAX; Focus software, Tucson, Arizona, USA) using biometrical information of anterior and posterior surfaces of the cornea obtained from OCT measurements and the IOL geometry obtained from noncontact profilometry (more detail will be described in the Zemax routines sections of Section 2.6.1 and Chapter 3). To calculate the position of the reflex on the different surfaces, routines were written in ZEMAX to search the rays that, following reflection from a surface, exit the eye parallel to the optical axis. While the standard Purkinje method studies the image created by all the rays passing through the pupil, the spots visible in en-face OCT images are created by a few rays. Weighting coefficients: A-G depend on the individual anterior segment geometry to calculate these coefficients. No tilt and no decentration $(\alpha=0$ and $d$ $=0$ ) were set in the computer eye model, and the Purkinje-like reflex positions were estimated for different rotation angles. A linear fit of the displacement of the reflex provided the value of the coefficients $E, F$, and $G$. The same procedure was repeated for A and $B$ (setting $\beta=0$ and $d=0$ ) and $C$ and $D($ with $\beta=0$ and $\alpha=0$ ) (Barry, Dunne et al. 2001).

Eye rotation $(\beta)$, lens tilt $(\alpha)$ and decentration $(d)$ were obtained from the inversion in Eq.2.5, using the positions of the experimental reflexes (PI, PIII, and PIV).

$$
\begin{array}{ll}
\beta=\frac{P I}{E} & - \text { Rotation angles } \\
\alpha=\frac{\beta \times(\mathrm{D} \times \mathrm{F}-\mathrm{C} \times \mathrm{F})+\mathrm{C} \times \mathrm{PIV}-\mathrm{D} \times P I I I}{C \times B-D \times A}-\text { Tilt angles } \\
\mathrm{d}=\frac{P I I I-\beta \times F-\alpha \times A}{C} & - \text { Decentration }
\end{array}
$$

Although the standard Purkinje images and the Purkinje-like reflections in OCT en-face images are different, the deviations between the Purkinje images represented in the computer eye model (using $\beta, \alpha$ and $d$ calculated) with the en-face OCT Purkinje-like images (for PI, PIII, and PIV) were always below $20 \mu \mathrm{m}$. Chapter 3 will present tilt and decentration data in five eyes of four patients obtained using the Purkinje-like method developed in this thesis. 


\subsection{Total aberration measurement: Laser Ray Tracing (LRT)}

\subsubsection{LRT setup}

Total wave aberrations are measured using a custom Laser Ray Tracing setup, described in detail in previous studies from VioBio (Barbero, Marcos et al. 2003, Dorronsoro, Barbero et al. 2003, Llorente, Diaz-Santana et al. 2003, Llorente, Barbero et al. 2004). The LRT consists of three channels: Illumination channel (incoming rays), Pupil and Retinal channel (outgoing) and Fixation channel (shown in Figure 2.9 (b) the scheme of the custom-LRT).

Two possible wavelengths could be selected between two diode lasers (green light: $532 \mathrm{~nm}$, Brimrose, Baltimore, MA, USA; infrared: 786 nm, Schäfter+Kirchhoff GmbH, Hamburg, Germany) for the Illumination channel. The measurements reported in this thesis are only IR. Maximum energy exposure is $7.6 \mu \mathrm{W}$. The laser beam samples the pupil in 37 samples typically, although the sampling pattern and density can be changed. The XY scanner (mod. 6210, Cambridge Technologies, Lexington, USA) consists of two rotating mirrors that deflected the incoming unexpanded laser pencil. That composes the sequential sample pattern with collimating lenses (with focal lengths of $50.8 \mathrm{~mm}$ ). The sampling pattern is adjusted by software to fit the subject's pupil.

In the Retinal channel (outgoing), the charge couple device (CCD) camera ( 8 bits, $60 \mathrm{~Hz}$ (video), $646 \times 485$ pixels, pixel size: $7.4 \mu \mathrm{m} \times 7.4 \mu \mathrm{m}$, Model XC-55, Sony Corp., Tokyo, Japan) records the corresponding aerial images of light reflected off the retina. In the Pupil channel, the pupil camera captures the corresponding image of the eye's pupil simultaneously with the spots reflected back from the retina on the retinal CCD (12 bits, 30 frames per second with $2 \times 2$ binning, $1024 \times 1024$ pixels, pixel size: $14 \times 14 \mu \mathrm{m}$, Model 1M15, Dalsa, Waterloo, Canada).

Prior to the measurement, the patient adjusts his/her subjective refraction using a Badal system from $-12 \mathrm{D}$ to $+12 \mathrm{D}$ in the Fixation channel, while viewing a stimulus on a minidisplay placed on a retinal conjugate plane. Ray aberrations are obtained by estimating the deviations of the centroids of the retinal spots images corresponding to each entry pupil location with respect to the reference (chief ray). 


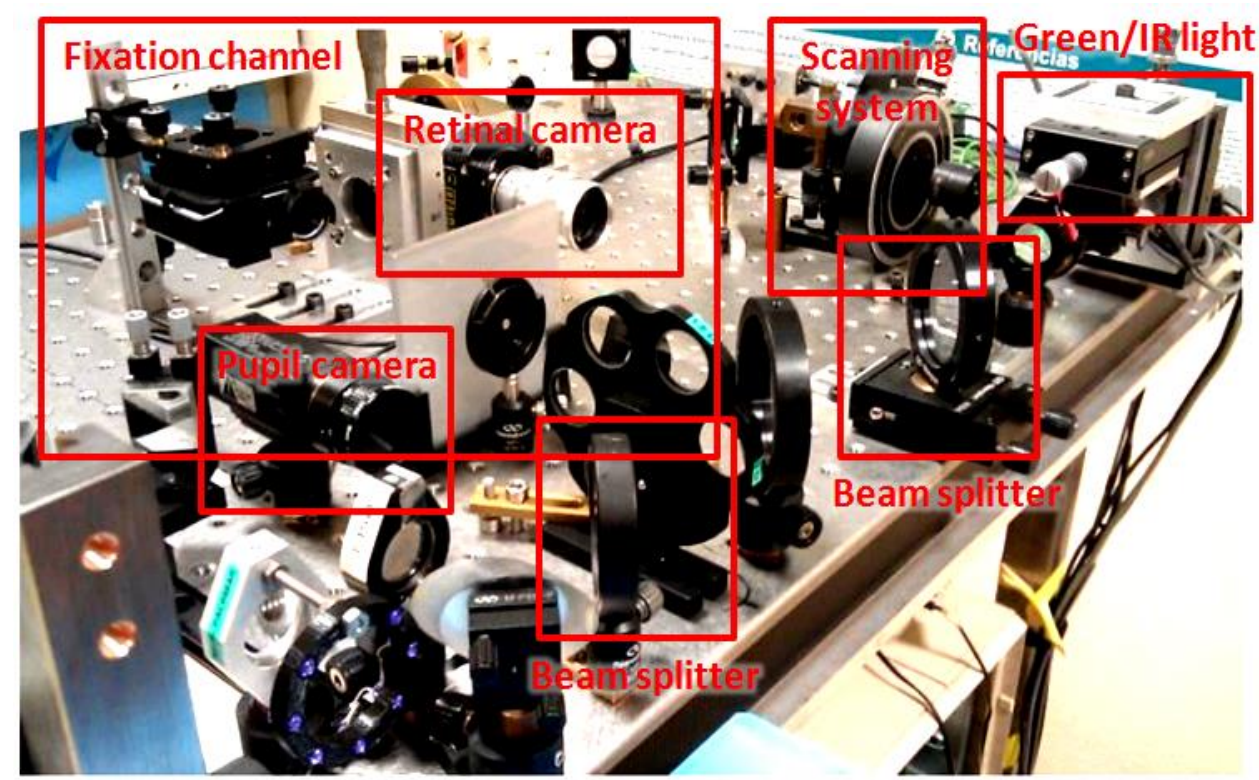

(a)

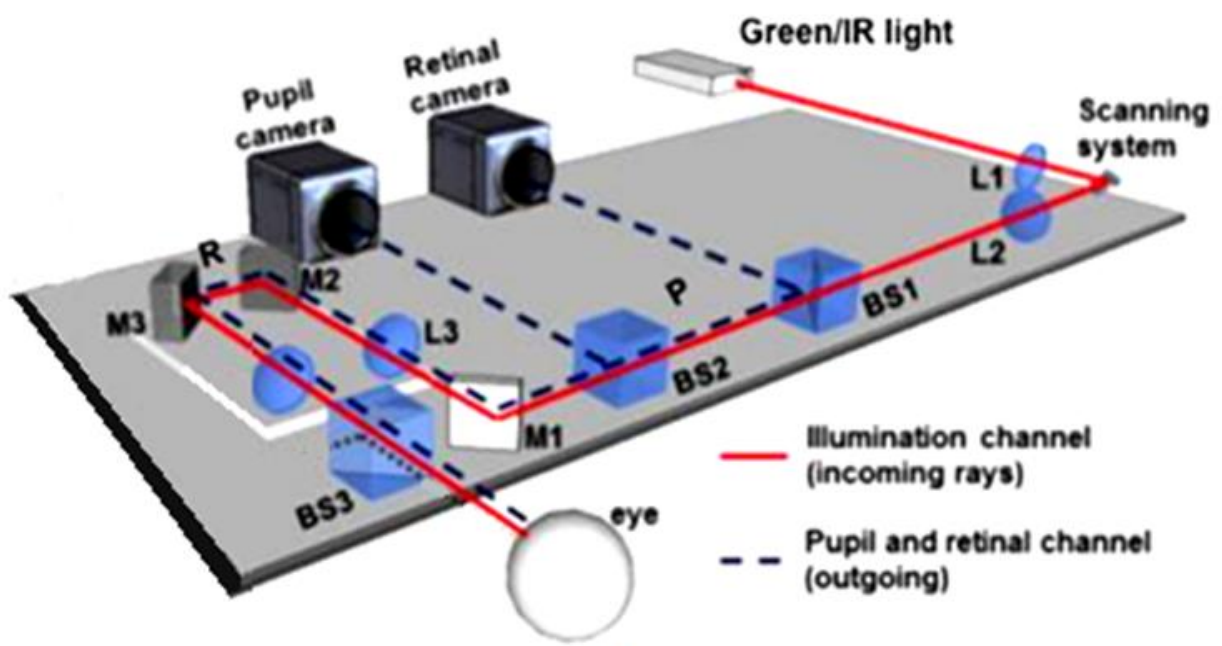

(b)

Figure 2.9 (a) Custom Laser Ray Tracing setup at the VioBio lab (Instituto de Óptica, "Daza de Valdés"). Retinal camera captured the light reflected back from the retina. Pupil camera captured the corresponding image of the eye's pupil simultaneously with the retinal spots on the retinal CCD. (b) Scheme of the custom-LRT modified from (Perez-Merino, Birkenfeld et al. 2014) (red solid line: illumination channel; dark blue dash line: Pupil and retinal channel.) 


\subsubsection{Control and analysis software}

The control and analysis software of the LRT was previously developed in our group by Lourdes Llorente and Carlos Dorronsora thesis. The control software was written in Visual Basic (Microsoft Corp., USA, front panel shown in Figure 2.10 (a)) and combined with Matlab (Mathworks, Natik, MA) scripts, which allowed changing the sampling pattern easily, e.g. scanned pupil diameter, sampling arrangement and density, and synchronizing the scanner, shutter and cameras for the calibrations and measurements.

The ocular aberrations are obtained from the set of aerial images by an analysis program developed in Matlab (Mathworks, Natik, MA). As showing in Figure 2.10 (b), the departure of rays from their ideal positions at the image plane is calculated (transverse aberration) from each aerial image and corresponding centroid, and the wave aberration is then estimated by fitting the transverse aberration corresponding to each sampled point to the derivatives of the Zernike polynomials, using a least squares method (Cubalchini et al. 1979). Chapter 5 will present laser ray tracing aberrometry measurements on patients implanted with IOLs.

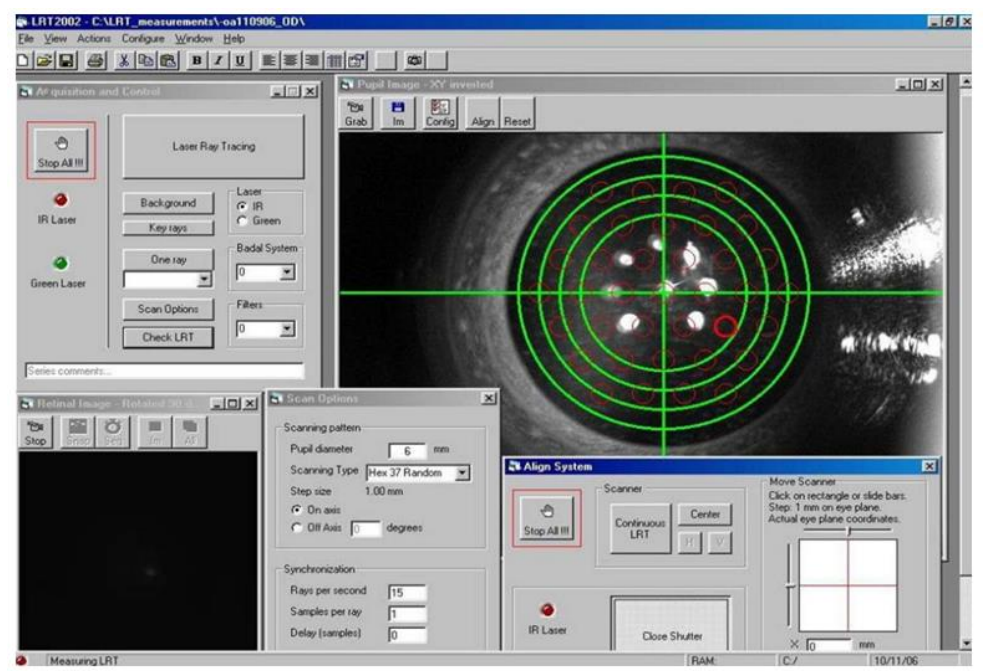

(a)

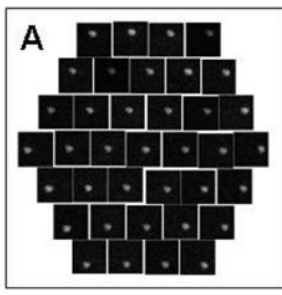

$543 \mathrm{~nm}$

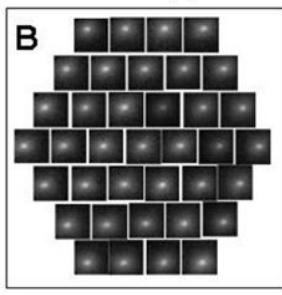

$786 \mathrm{~nm}$

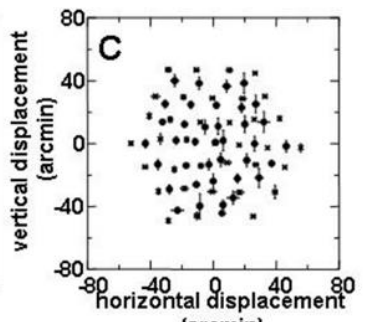

(arcmin)

(b)

Figure 2.10 (a) Snapshot of the control program developed by Carlos Dorronsoro (Top right and bottom left are the pupil and retinal images, respectively); (b) Example of aerial images obtained from LRT using green and IR light in Panels A and B. Panel C shows the corresponding spot diagram. Crosses and circles represent green and IR illumination respectively. (Llorente, DiazSantana et al. 2003) 


\subsection{Other techniques for validation}

\subsubsection{Pentacam: Scheimpflug camera for the validation of corneal topography}

The Pentacam images the anterior segment of the eye by a rotating Scheimpflug camera measurement. This rotating process allows for the rapid capture of images in different meridians, and therefore three-dimensional elevations. A commercial Pentacam Scheimpflug topographer (Oculus Optikgeräte GmbH, Wetzlar, Germany), as shown in Figure 2.11(a), has been used to get corneal topographies. In this thesis, anterior and posterior corneal elevation maps, which are introduced into customized computer eye models, were obtained from 3D OCT, as described in Section 2.1.2 and in earlier publications (Perez-Merino, Velasco-Ocana et al. 2015), and were fit by 6th order Zernike polynomials. Quantitative information of anterior and posterior corneal topographies provided by Pentacam commercial software in Figure 2.11(b), using conventional clinical protocols (corneal reflex centration, 25 cross-sections, 50 semi-meridians, chin and forehead rest stabilization) will be used for the validation of corneal topography.

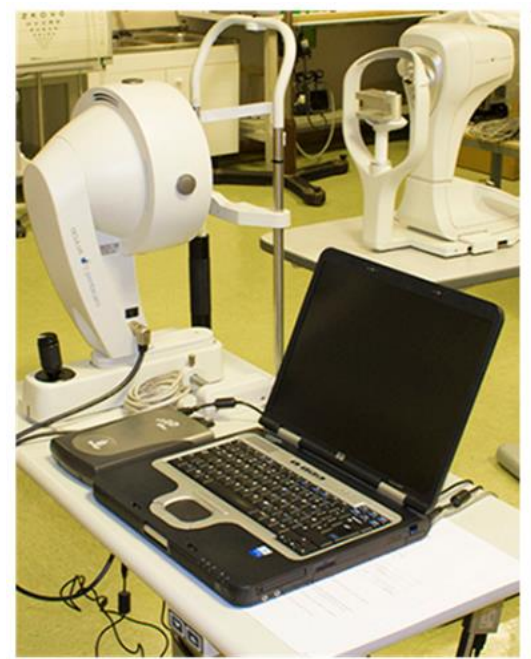

(a)

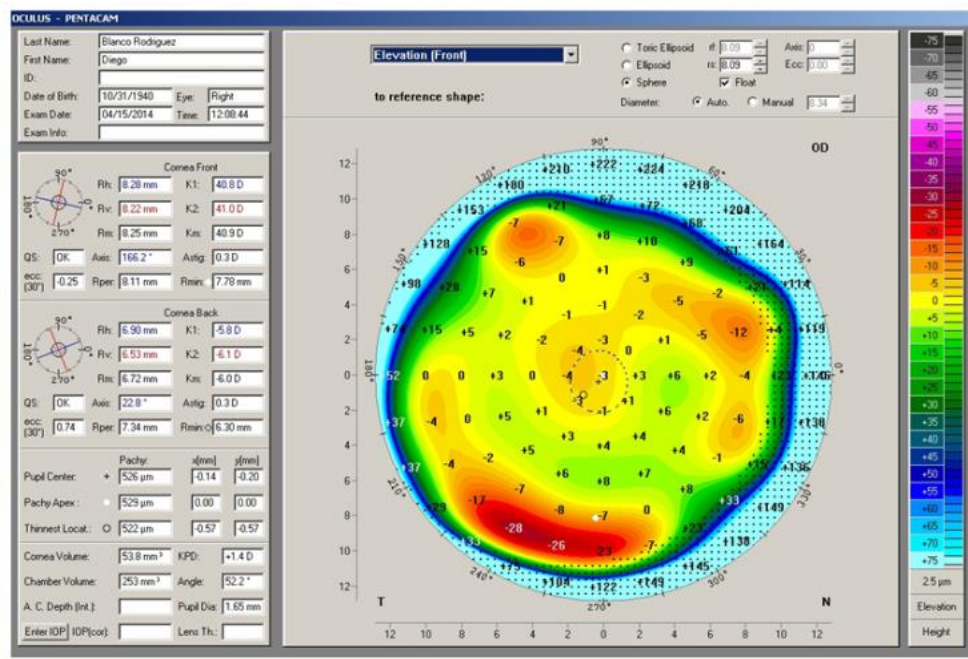

(b)

Figure 2.11 (a) Commercial Pentacam Scheimpflug topographer (Oculus Optikgeräte GmbH, Wetzlar, Germany) at the VioBio lab (Instituto de Óptica, "Daza de Valdés") (b) interface of commercial Pentacam Scheimplug topographer

Figure 2.12 shows OCT-based anterior and posterior corneal elevation maps in 3 eyes showing a good correspondence with anterior and posterior corneal elevation maps obtained with a commercial Scheimpflug topographers from a previous study (Ortiz, Siedlecki et al. 2011). Discrepancies of radii of curvature from Scheimpflug topography and sOCT were between 1 and 2\%. Chapter 5 will report OCT-based anterior and posterior corneal elevation in three eyes of two patients implanted with IOLs, and corneal maps from Pentacam obtained on the same eyes using the same pupil reference for comparison. 

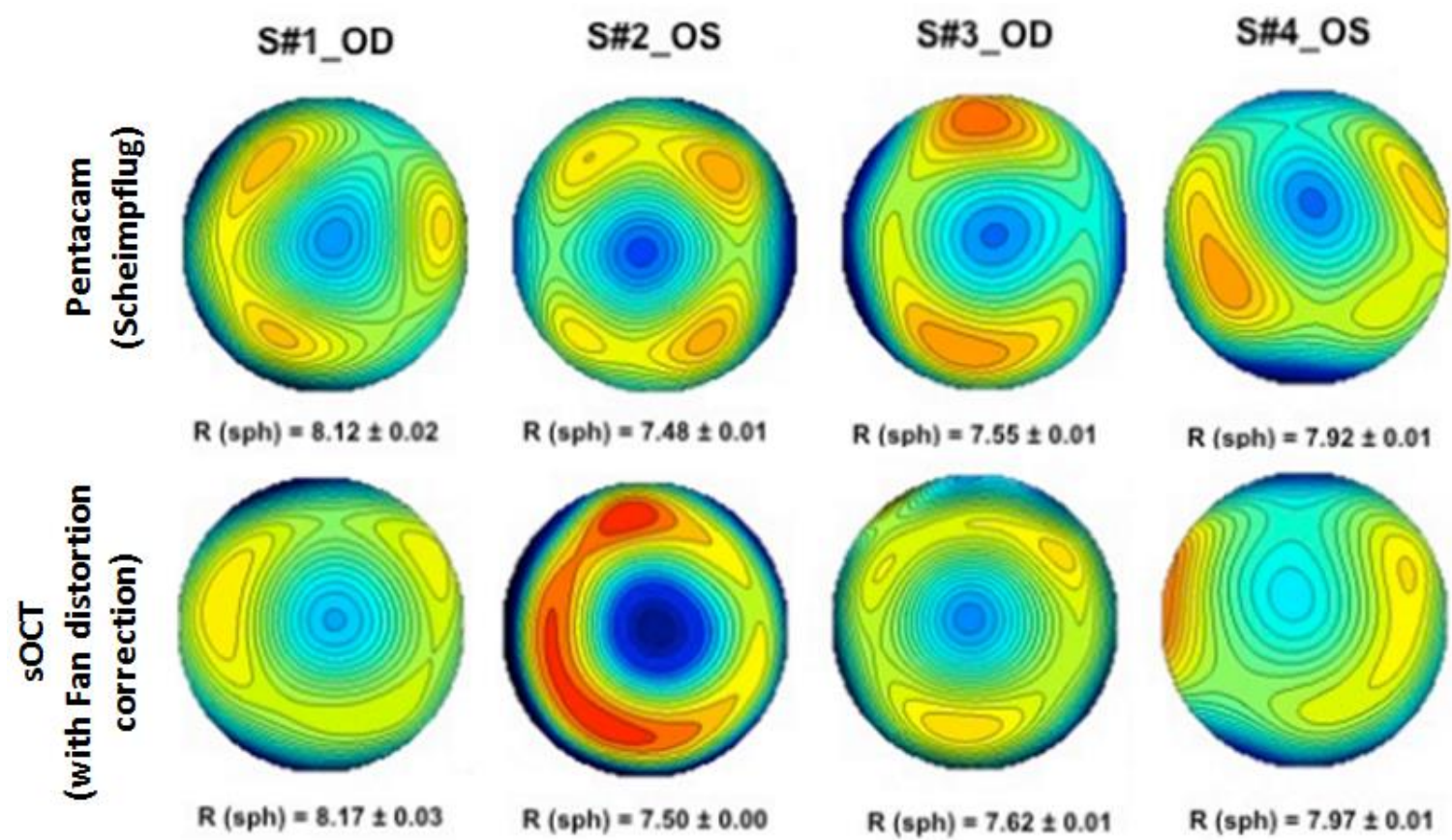

Figure 2.12 Corneal elevation maps obtained in 4 eyes obtained from different instruments (relative to the best fitting sphere). $\mathrm{R}=$ radii of curvature of the best fitting sphere (from fits to sphere quadrics), modified from (Ortiz, Siedlecki et al. 2011).

\subsubsection{Sensofar PLu2300: Non-contact profilometry for the validation of IOL geometry ex-vivo}

Profilometry and OCT are both efficient measuring instruments used to measure a surface's profile to quantify its geometrical information. The geometry of the IOLs was characterized ex vivo using microscopy-based noncontact profilometry (Sensofar, PLu2300, Barcelona, Spain), as shown in Figure 2.13 (a), using a 100x microscope objective, in order to have the values of reference. To ensure the accuracy of measurements, each surface measurement is repeated at least five times and the IOL is rotated in different directions between measurements to ensure that the measurement results are not entirely consistent. Repeated measurements on these lenses revealed repeatability in the apex measurement of $5 \mu \mathrm{m}$. Both anterior and posterior lens surface topographies were measured in a $5.5 \times 5.5 \mathrm{~mm}$ range (1840 points, with $0.1-\mu \mathrm{m}$ nominal vertical precision (on rigid samples), and $0.11 \mathrm{~mm}$ horizontal precision). De-hydration effects on these IOLs with high water content (25-26\%) decrease the effective precision.

The IOL surface profiles were fitted to conicoids (Section 2.1.2, Eq.2.2) (Perez-Escudero, Dorronsoro et al. 2010) using custom routines written in MATLAB. The profilometric data processing comprises the flowing steps: (1) importing the raw data (shown as Figure 2.13 (b)), (2) noise removal, (3) removal of potential tilts of the mounted IOL in the profilometric measurements (Perez-Escudero, Dorronsoro et al. 2010)(shown as Figure 2.13 (c)). Noncontact profilometry was used in this thesis to obtain the geometry of the IOLs ex vivo (anterior and posterior IOL radii of curvature and thickness). 


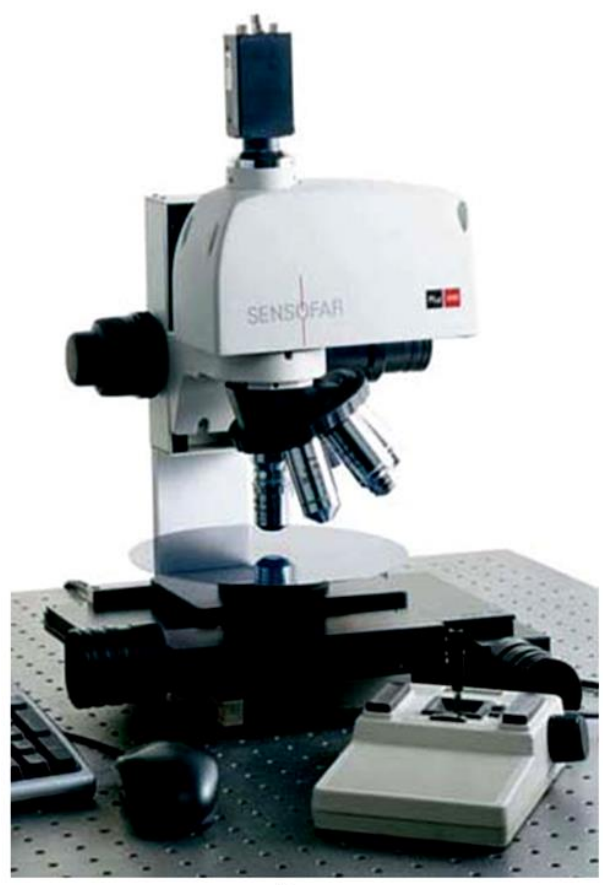

(a)

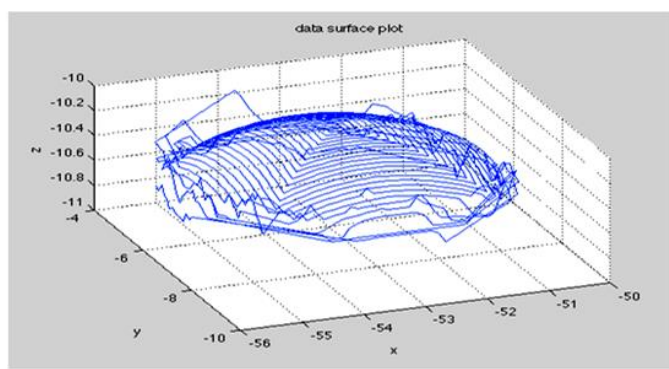

(b)

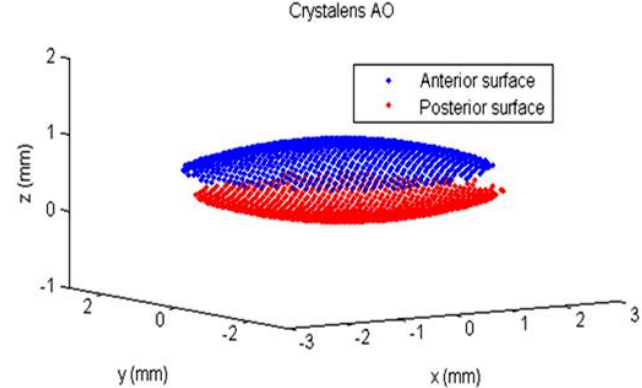

(c)

Figure 2.13 (a) Commercial non-contact profilometry (Sensofar, PLu2300, Barcelona, Spain) (b) profilometry raw data with tilt and noise (c) quantified anterior and posterior surfaces of intraocular lens with customized algorithm

\subsubsection{Water-cell physical model eye for the validation of eye rotation, IOL tilt and decentration}

The cornea and IOL form a compound optical system, but they are not a centered optical system, with the fovea tilted with respect to the optical axis. The IOL is both tilted and decentered with respect to the pupillary axis to compensate for the aberration of the cornea, so the results of this simulation result are to demonstrate key points for the further research.

The physical model eye in Figure 2.14 had been previously built at VioBio Lab (de Castro et al., 2007) specifically for the validation of eye rotation, IOL tilt and decentration, where nominal values could be set. The water-cell physical model with a PMMA contact lens (AR3 Vision, Madrid, Spain) simulating a spherical cornea $(7.80 \mathrm{~mm}$ and $6.48 \mathrm{~mm}$ anterior and posterior radii of curvature, respectively, with $0.5 \mathrm{~mm}$ central thickness), with parameters similar to the Gullstrand eye model and an IOL (aspheric biconvex IOL) mounted on a XYZ micrometer stage and rotational stage was used for testing. The watercell eye model is similar to that reported in previous work (de Castro, Rosales et al. 2007, Ortiz, Perez-Merino et al. 2013), except for the IOL. We used the same A-IOL type (Crystalens AO 23.5 D) in the artificial eye that was implanted in the patients under test. Radii of curvature, axial biometry, and refractive indices in the physical model eye can be found in Table. 2.1. The thickness of the IOL (Crystalens AO 23.5 D) was measured using the profilometry system mentioned in Section 2.3.1.

This artificial eye was used in validations of the tilt and decentration measurement method developed in this thesis. Measurements were obtained for the artificial eye with the lens in air (i.e., cell not filled in with water) and in water. The IOL was tilted inside the water-cell 
eye from -5 to 5 degrees in 1-deg steps and decentered from -1.9 to $1.9 \mathrm{~mm}$ in $0.6-\mathrm{mm}$ steps.
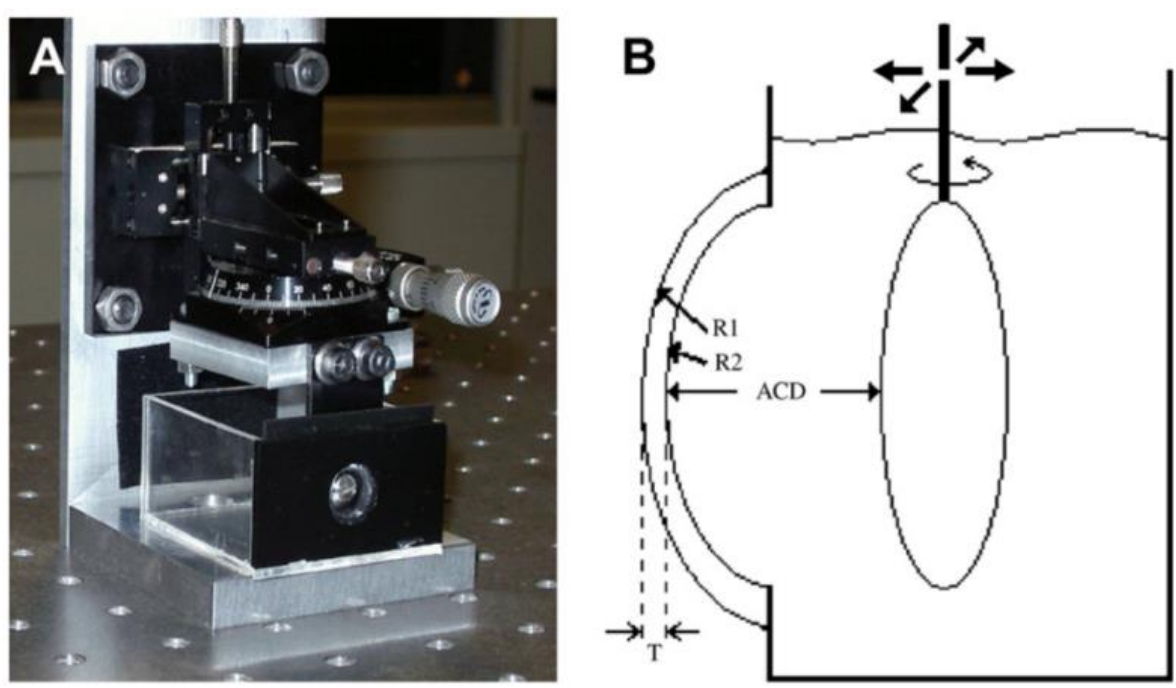

Figure 2.14 (a) Photograph and (b) schematic diagram of the physical model eye developed for this study. A PMMA contact lens simulates the cornea. The IOLs are positioned with an XYZ micrometer stage and rotational stage (de Castro, Rosales et al. 2007).

Table 2.1 Radii of curvature, axial biometry, and refractive indices in the physical model eye

\begin{tabular}{lc}
\hline \hline Eye \# & Artificial eye model \\
\hline Anterior corneal radius of curvature $(\mathrm{mm})$ & 7.800 \\
Posterior corneal radius of curvature $(\mathrm{mm})$ & 6.480 \\
Anterior intraocular lens (IOL) radius of curvature $(\mathrm{mm})$ & 7.781 \\
Asphericity Q of anterior IOL radius & -1.35 \\
Posterior IOL radius of curvature $(\mathrm{mm})$ & -7.937 \\
Asphericity Q of posterior IOL radius & -2.356 \\
Corneal thickness & 0.500 \\
Anterior chamber depth ACD (mm) & $3.825^{\mathrm{a}} / 4.124^{\mathrm{b}}$ \\
IOL thickness (mm) & 1.100 \\
Corneal refractive index & 1.450 \\
Aqueous humor refractive index & 1.346 \\
IOL refractive index & 1.440 \\
\hline \hline
\end{tabular}

${ }^{\mathrm{a} E y e ~ m o d e l ~ i n ~ a i r . ~}$

${ }^{b}$ Eye model in water. 


\subsubsection{Custom computer model eyes}

OCT-based elevation data from both anterior and posterior corneal surfaces, anterior segment distances, IOL geometry, IOL alignment, Eye rotation angle lambda $\lambda$ and axial length from IOLMaster (Carl Zeiss AG, Jena, Germany) were exported to ZEMAX, where the custom model eyes were simulated. The elevation data obtained from OCT distortioncorrected surfaces were fit to a Zernike polynomial expansion (up to 6th order) described in Section 2.1.2 and Eq.2.3. The object (light source) was set at infinity. Refractive indices of the cornea, aqueous humour, and vitreous humour were 1.376, 1.337 and 1.337 respectively at $555 \mathrm{~nm}$. Knowledge of the Abbe number of the IOL material allowed extrapolating the index of refraction from the visible to other wavelengths (i.e., IR typically used in wavefront sensing).

Abbe number $v(\lambda)=\frac{n(\lambda)-1}{n_{F^{\prime}}-n_{C^{\prime}}}$

Where $n_{F^{\prime}}$ and $n_{C^{\prime}}$ are the refractive indices of the material at the wavelengths of the F' and $C^{\prime}$ spectral lines (486.1 $\mathrm{nm}$ and $656.3 \mathrm{~nm}$ respectively). Wave aberrations were calculated in the pupil plane, placed at the Anterior Camber Depth (ACD) distance from the posterior corneal surface, by tracing a rectangular array grid of $64 \times 64$ collimated rays, with wavelength $555 \mathrm{~nm}$, through the eye surfaces within the central $5-\mathrm{mm}$ pupil diameter area. Figure 2.15 illustrates the computation of aberrations from OCT data, i.e. ray tracing calculation on OCT distortion-corrected corneal and IOL surfaces. Data was analyzed in terms of Zernike coefficients and retinal image quality metrics (MTF and Visual Strehl) (Applegate, Marsack et al. 2003, Cheng, Thibos et al. 2003, Guirao and Williams 2003, Applegate, Marsack et al. 2006, Iskander 2006). More detail will be described in Section 2.4.3.

Custom computer model eyes were used in this thesis to simulate aberrations, allow understanding the relative contribution of optical geometrical and surgically-related factors to image quality, and improve selection of the IOL to be implanted, in particular, for the calculation of IOL power with higher accuracy than regression-formulae. 
Chapter 2

Experimental methods, computational techniques and other types of validation

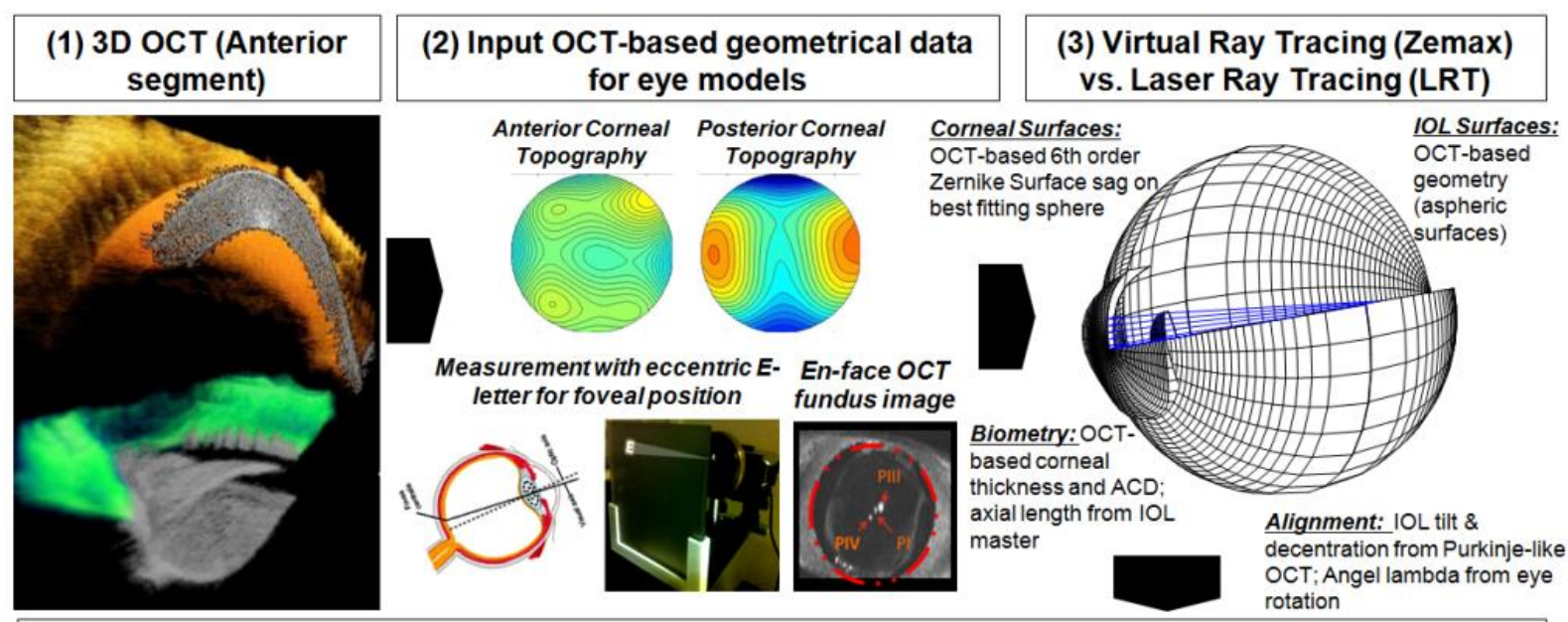

(4) Virtual Ray Tracing (Zemax) vs. Laser Ray Tracing (LRT)
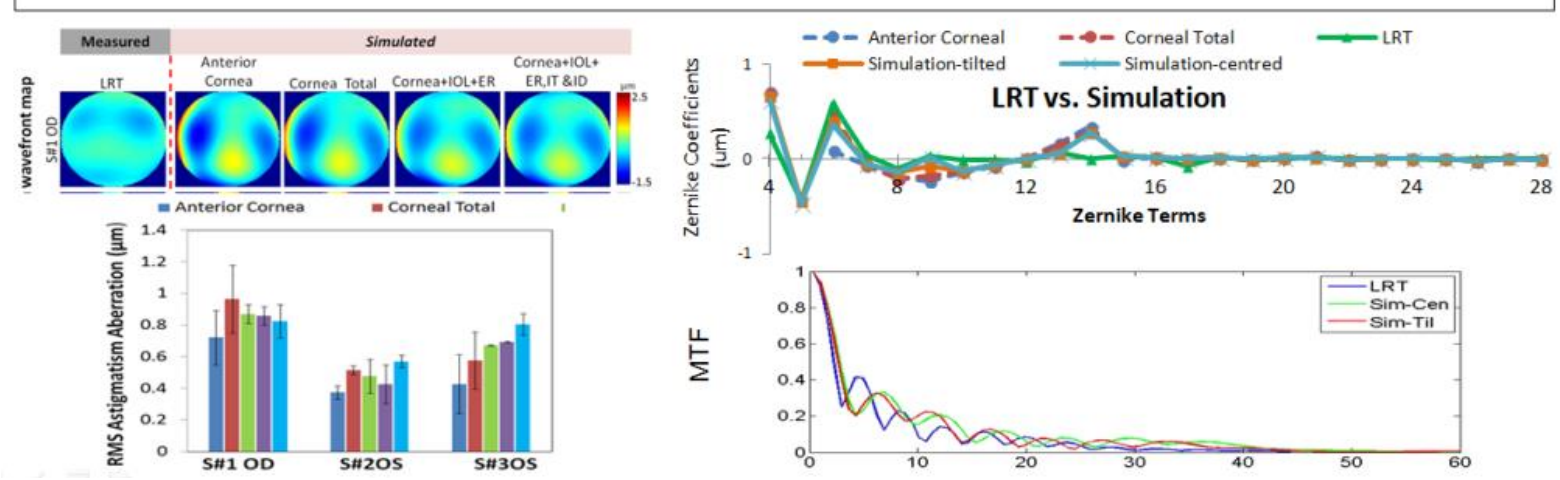

Figure 2.15 Illustration of the computation of aberrations using quantitative OCT geometrical data in a customized computer pseudophakic eye model 


\subsection{Optical quality metrics}

\subsubsection{Spot diagrams}

Since the advent of ray tracing computational programs software (such as ASAP (Breault Research Organization, Inc., Tucson, AZ), ZEMAX (Radiant ZEMAX; Focus software, Tucson, AZ), Code V (Optical Research Associates, Pasadena, CA) or OSLO (Lambda Research Corporation, Littleton, MA)), it is possible to trace very quickly a large number of rays in the entry pupil of human eye, and the computed intersections of these rays with the receiving ocular surfaces provide a 'spot diagram' which gives a good deal of evaluating the image quality numerically.

The spot diagram is important in predicting the performance of lenses from an analysis of their designs. The light rays can be traced by simulations in Zemax, as demonstrated in the spot diagrams from Figure 2.16(a) or experimentally from Laser Ray Tracing measurements, as shown in Figure 2.16(b). The geometric spot (GEO) radius for each field point are the deviations from the reference point, and the reference points are defined as either the chief rays at the primary wavelength, the centric of all rays traced or the middle of the spot cluster. In other words, the GEO radius is the radius of the centric circule at the reference point which encloses all the rays. The information of the spread of all rays can be showed by the RMS spot radius, which depends upon each ray of light; while the GEO radius only gives information related to the ray which is farthest from the reference point.

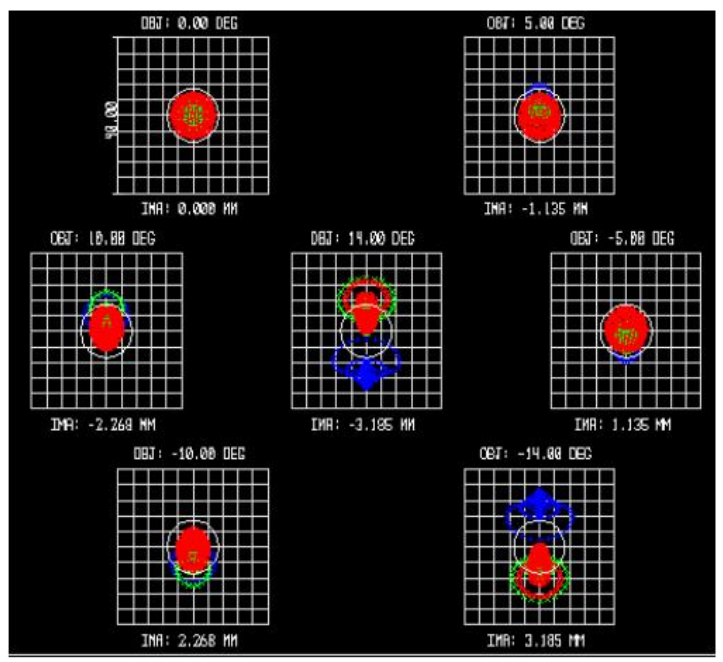

(a)

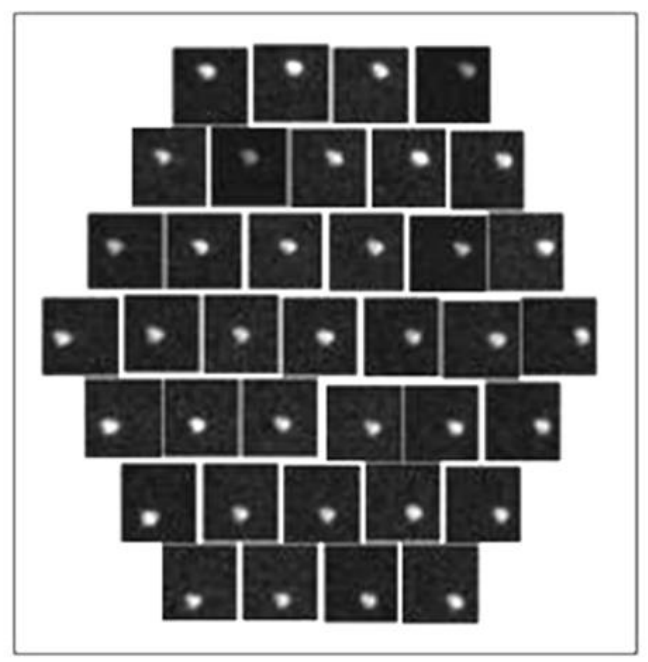

(b)

Figure 2.16 (a) Example of RMS radius and max GEO radius report of spot diagram from -14 to 14 degrees' field angle around horizontal axis with three wavelengths (Blue, green and red present the wavelengths of $486.1 \mathrm{~nm}, 587.6 \mathrm{~nm}$ and $656.3 \mathrm{~nm}$, respectively) in Zemax; Circle in white is Airy Disk; (b) Example of spots diagram images obtained from LRT at $543 \mathrm{~nm}$ wavelength 


\subsubsection{Zernike coefficients and RMS wavefront error}

Aberrations are departures of the performance from the predictions of paraxial approximations in an optical system, which leads to blurring of the image. The most important aspect of aberration analysis is the optical path difference (OPD) between the chief ray and other rays originating from the same object point at the focus location by using Snell's laws of refraction and Fermat's principles in Figure 2.17(a). While the wavefront aberration form (shown in Figure 2.17(b)) is more directly related to the physical fundamentals determining image quality, ray aberration form (shown in Figure 2.17(c)) offers a more convenient graphical interface for the initial evolution of the optical quality for the whole system.

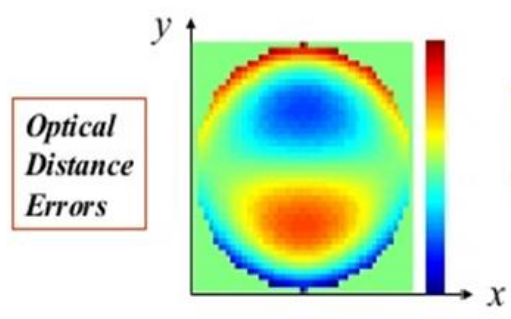

(a)

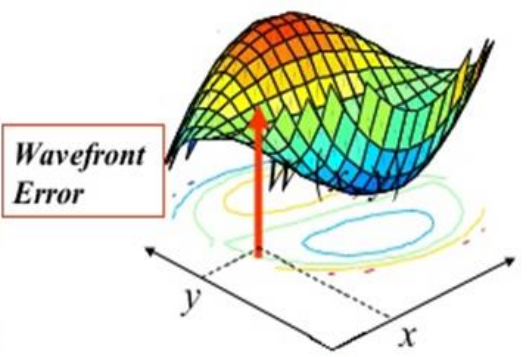

(b)

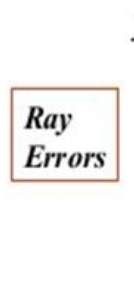

Figure 2.17 (a) Optical Distance Errors; (b) Wavefront Error; (c) Ray Errors

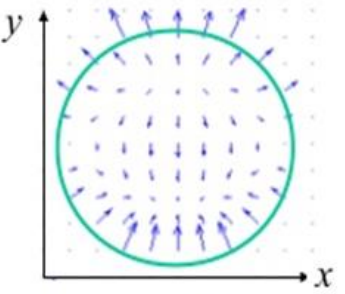

(c)

The wave aberrations of a general optical system can be described mathematically by a polynomial series. Zernike polynomial expansion, mentioned before in the section on elevation based topography (Eq.2.3, in Section 2.1.2), has also become the standard for representing wave aberration data because the polynomials form an orthogonal set over a unit circle, and aberrations are usually in reference to circular pupils (Malacara 1990, Mahajan 1994). This makes them suitable for accurately describing wave aberrations as well as for data fitting.

Monochromatic aberrations include piston, tilt, astigmatism, defocus, coma, spherical aberration (shown in Figure 2.18). Spherical aberration (arising from the asphericity of the optical surfaces), coma (mainly associated to local irregularities, tilt and decentration of ocular surfaces), and astigmatism (mainly produced by toricity of ocular surfaces and tilt of the lens) are the three dominant aberrations which result from deviations in the wavefront form from spherical, and spherical aberration is the only aberration independent of the eye rotation, IOL tilt and decentration. 


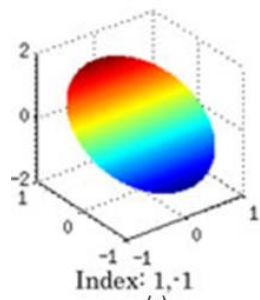

(a)

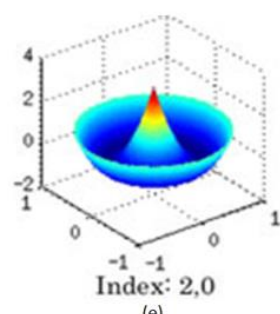

(e)

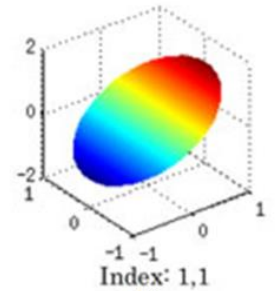

(b)

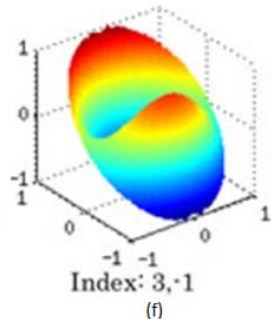

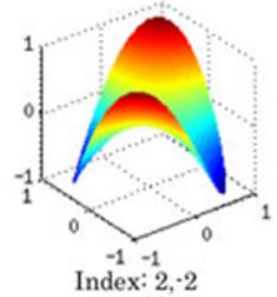

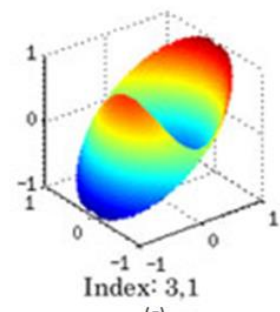

(g)

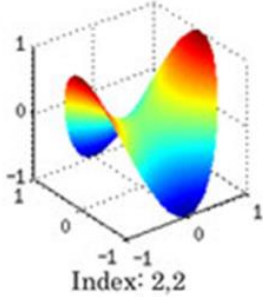

(d)

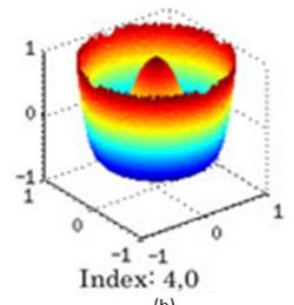

(h)

Figure 2.18 Wavefront aberration: 1st order aberrations: (a) Vertical Tilt (b) Horizontal Tilt; 2nd order aberrations: (c) Oblique Astigmatism (d) Vertical Astigmatism (e) Defocus; 3rd order aberrations: (f) Vertical Coma (g) Horizontal Coma; 4th order aberration: (h) Spherical Aberration

The interactions between multiple modes are necessarily complex, as is required to describe potentially very complicated wavefront. The RMS is also a common descriptor of the deviation of the wavefront from a prefect plane wave, which is defined as the root square of the variance of the wave aberration and is typically used as the global metric for the optical quality (Applegate, Hilmantel et al. 2000, Applegate 2003). The total wavefront RMS is equal to the square root of the sum of the squares of the individual Zernike coefficients (Eq. 2.7). Similarly, the Zernike coefficients of modes describing the lower order aberrations may be used to generate a lower order wavefront RMS value and the Zernike coefficients of modes describing the higher order aberrations may be used to generate a higher order wavefront RMS value. The calculation of RMS error can be done either individually or grouped arbitrarily.

$R M S=\sqrt{\sum_{n, m}\left(C_{n}^{m}\right)^{2}}$

Where $C_{n}^{m}$ is the Zernike coefficient corresponding to the order $n$ and frequency $m$. Zernike polynomials are used in this thesis both to represent wave aberrations as well as to describe the elevations of ocular surfaces (with respect to the best fitting sphere).

\subsubsection{Modulation transfer function, Strehl and visual Strehl}

The image of a point object formed by the optical system is the Point Spread Function (PSF). The PSF for a perfect optical system is the Airy disk, and is calculated as the squared magnitude of the inverse Fourier transform of the pupil function (Maeda 2003). The Optical Transfer Function (OTF) is defined as the Fourier transform of the PSF, which is limited to the effect of the system PSF on imaging and is defined as (Maeda 2003).

$O T F(v)=\int P S F(x) e^{i v x} d x$ 
Where $\mathrm{x}$ is the spatial coordinate in the image plane, and $v$ is spatial frequency. The Modulation Transfer Function (MTF) is the modulus of the OTF, and represents the decrease in the contrast as a function of the spatial frequency (Maeda 2003).

$$
M T F=|O T F|
$$

With RMS wavefront errors, the generalized MTF can be assumed to also represent the averaged contrast drop for a given RMS error for asymmetrical aberrations, like coma, astigmatism and irregular high order wavefront of the actual system. Maeda et al. (Maeda 2003) generated PSFs and MTFs of wave aberration corresponding to each Zernike mode up to the 6th order with a $4 \mathrm{~mm}$ pupil size, and $0.53 / 0.2$ wavelength RMS wavefront error for PSF and MTF respectively (shown as Figure 2.19), to show how the various Zernike modes affect the PSF and MTF relative to an unaberrated system.

The Strehl Ratio (SR) is a measure of the quality of optical image formation, originally proposed by Karl Strehl (1864-1940), and describes the reduction in the peak power of the point image basically. In the spatial domain, it can be calculated directly from the PSF. SR is defined as the maximum value of the PSF in the presence of aberrations, normalized by the maximum of the diffraction limited PSF (ranging from 0 to 1 , with 1 defining a perfect optical system) for the same pupil size. In the frequency domain, the SR is computed as the volume under the MTF of an aberrated system normalized by the diffraction-limited MTF, for the same pupil diameter.

$$
\text { Strehl Ratio }=\frac{P S F_{\text {aberrated }}(\mathrm{x}, \mathrm{y})}{P S F_{\text {ideal }}(\mathrm{x}, \mathrm{y})}
$$

As the Strehl Ratio includes in the calculation spatial frequencies of the MTF beyond those relevant to the visual system, a new metric was introduced to adapt the definition to visual optics (Visual Strehl ratio (VS)). VS has been shown to hold the highest correlation variance against subjective acuity (Cheng, Thibos et al. 2003), and showed the highest predictability (Buhren, Pesudovs et al. 2009). The Visual Strehl is computed as the volume under the visual MTF, which is obtained by overlapping the MTF with the inverse of a general neural transfer function and normalizing to the diffraction limit. The VS was based on calculated OTF across all spatial frequencies. In this thesis, we have used VS as our optical quality metric, considering high order aberration from 3rd order to 6th order with/without Astigmatism. Depth of focus was also estimated with a range of $\pm 1.5 \mathrm{D}$ with $0.125 \mathrm{D}$ defocus steps. 


\subsection{Ex vivo and in vivo measurements}

\subsubsection{Lens specimens, preparation and experimental protocol}

This thesis involved measurements on donor lenses (a total of 36 eyes) and on patients before and after implantation of IOLs (a total of 11 eyes). Thirty-six isolated human crystalline lenses from twenty-eight human donor eyes were obtained from the BST Eye bank (Banc de Sang i Teixits, Barcelona, Spain). In all cases the cornea had been previously removed by the eye bank for clinical use. All eyes were shipped in sealed vials at $4^{\circ} \mathrm{C}$ and moisturized in preservation medium (DMEM/F-12, HEPES). Crystalline lenses with evident cataract formation and lenses with obvious surface damage were exclusion from the study. Methods for securing human tissue were in compliance with the Declaration of Helsinki. The handling and experimental procedures, described in detail in a prior publication (Birkenfeld, de Castro et al. 2014), have been approved by the institutional Review Boards of BST and Consejo Superior de Investigaciones Científicas (CSIC). The post-mortem time varied between 1 and 3 days at the time of the experiment. A typical experimental session lasted 1-2 hours. Measurements on donor eyes are described in Chapter 4. 


\subsubsection{Patients}

Eleven cataract patients (fifteen eyes) were measured before and after cataract surgery (IOL implantation) in this thesis. The age range is from 66 to 84 years old. Standard phacoemulsification intracapsular cataract surgery had been performed by a single surgeon at Fundación Jiménez Díaz Hospital (FJD). The measurements performed on those patients started with an aberrometery designed in the group, and all measurements in pre- and postoperative patients were performed under mydriasis (phenylephrine 10\%).

Total wave aberrations were measured using a custom-developed LRT system. We use a commercial Scheimpflug image topographer Pentacam (Oculus, Germany) to get corneal topography as a reference, and an IOLmaster (Carl Zeiss AG, Jena, Germany) to obtain the axial length of the eye. Anterior segment geometry (cornea, iris and IOL) was measured using a custom-developed SD-OCT. LRT measurements were done under foveal fixation, and OCT measurements were done at with off-axis fixation that aligned the pupillary axis with the optical axis of the instrument. Experimental protocols were approved by Institutional Review Boards at FJD and CSIC. Patients signed informed consent after the nature of the study had been explained to them. All protocols followed the tenets of the Declaration of Helsinki. More detail could be found in Section 5.2.2.

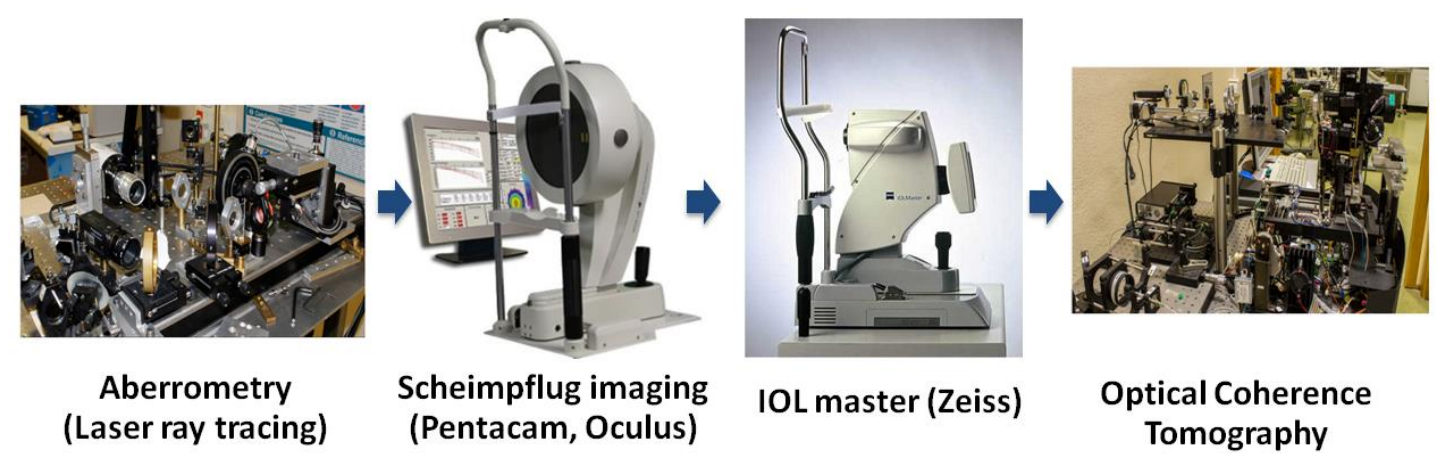

Figure 2.19 Process steps of patients' measurement: Aberrometry, Pentacam, IOLmaster and Optical Coherence Tomography 


\subsection{Virtual ray tracing calculations}

\subsubsection{Zemax routines}

Sequential ray tracing was performed using commercial dedicated software ZEMAX (Radiant ZEMAX; Focus software, Tucson, Arizona, USA). Several variables must be predefined for the ray tracing: 1) Light/object source, the field angle of the ray entering the optical system. 2) Position, shape type of image surface and coordinate break. 3) Stop surface and size, which defines the entrance-exit pupil size and position. 4) Wavelength used for the ray tracing.

Zemax based computations were used in this thesis for the following purposes:

\section{-Build full 3D OCT-based custom pseudophakic computer eye models}

The 3D Layout in Figure 2.20 shows visible light rays passing through the pupil in a personalized computer eye model. Corneal Surfaces are OCT-based 6th order Zernike Surface Sag on best fitting sphere and IOL surfaces are OCT-based geometry (aspheric surfaces) with IOL tilt and decentration from Purkinje-like OCT method. OCT-based biometry such as corneal thickness and anterior camber depth are also introduced into the customized eye model, with axial length from the IOLMaster. The corresponding study will be presented in Chapter 5 .

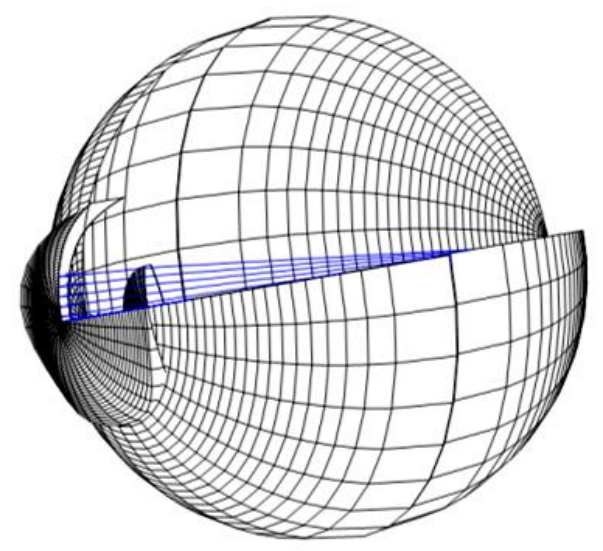

Figure 2.20 Solid Model 3/4 draw section of full-OCT based customized eye model. Corneal Surfaces: OCT-based 6th order Zernike Surface Sag on best fitting sphere; IOL Surfaces: OCTbased geometry (aspheric surfaces); Biometry: OCT-based corneal thickness and ACD; axial length from IOLMaster; Alignment: IOL tilt \& decentration from Purkinje-like OCT; Angle lambda from eye rotation.

-Estimation of weighting coefficients in Purkinje method tilt and decentration (see Section 3.2.5)

Eye rotation, IOL tilt and decentration were calculated from Purkinje-like methodology described in Section 2.1.3. Simulation routines were written in ZEMAX using biometrical information of anterior and posterior surfaces of the cornea obtained from OCT measurements and the IOL geometry obtained from noncontact profilometry (shown in 
Experimental methods, computational techniques and other types of validation

Figure 2.21): PI: Reflection from Cornea; PIII: Refracted through cornea, reflected at anterior lens, refracted back through cornea; PIV: Refracted to back of lens, reflected by posterior lens, refracted back to anterior cornea.

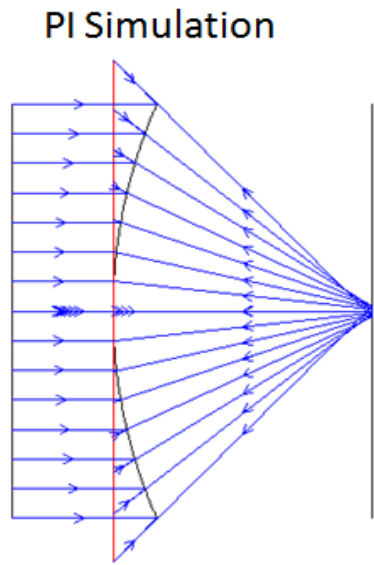

(a)

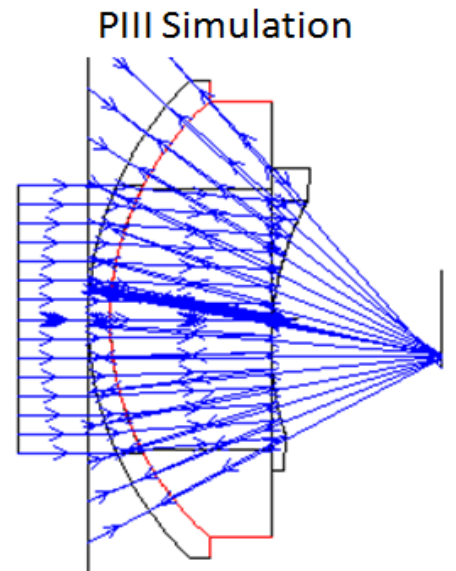

(b)

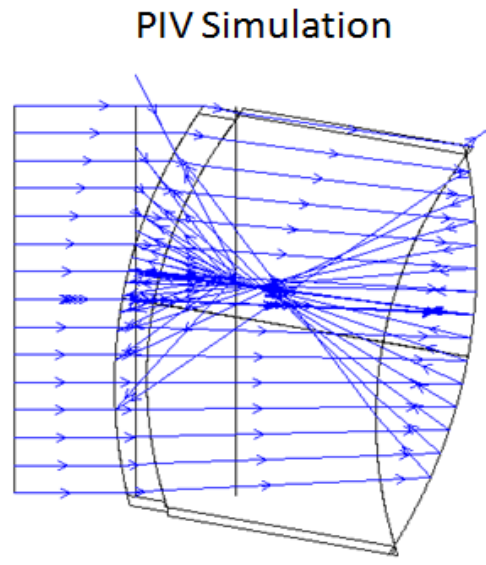

(c)

Figure 2.21 3D layouts of simulations in ZEMAX (a) PI (b) PIII (c) PIV

\section{- Calculations of high order aberrations in custom pseudophakic eye models, and comparison with high order aberration measurements.}

The wave aberration is reconstructed from a finite number of rays. The accuracy of the result depends on whether the sampling is sufficiently dense. Therefore, wave aberrations were calculated by tracing a rectangular array of grid $64 \times 64$ collimated rays through the eye surfaces within a central 5-mm pupil diameter area, at $555 \mathrm{~nm}$. A complete ray tracing procedure provides a discrete set of local measurements of the wave aberration. Zemax uses a modal reconstruction with a standard least squares algorithm fitting to a Zernike expansion in Zemax order, which will be transferred to OSA Zernike polynomial order. The corresponding study will be presented in Chapter 5. (MATLAB algorithm of shifting between Zemax order and OSA order will be described in Section 2.6.2).

\section{- Calculation of optimal IOL lateral position}

In order to identify optimal IOL centration, OCT-based ray tracing on a pseudophakic eye model has been used to control an optical system's primary structure control off-axis designated view, and estimated for the native tilt \& decentration, with simulated decentrations ranging from $-1-1 \mathrm{~mm}$ in $0.1 \mathrm{~mm}$ steps for $5 \mathrm{~mm}$ pupil diameters in the horizontal and vertical meridians with reference to the pupil center, as shown in Figure 2.22. The optical aberration of each simulation designated beam structure in terms of aberration structure, usually with defocus, spherical aberration, RMS astigmatism, RMS coma aberration, and other optical quality metrics in terms of VS as parameters. More detail about Optical quality metrics will be explained in Section 2.4. The corresponding study will be presented in Chapter 6 . 


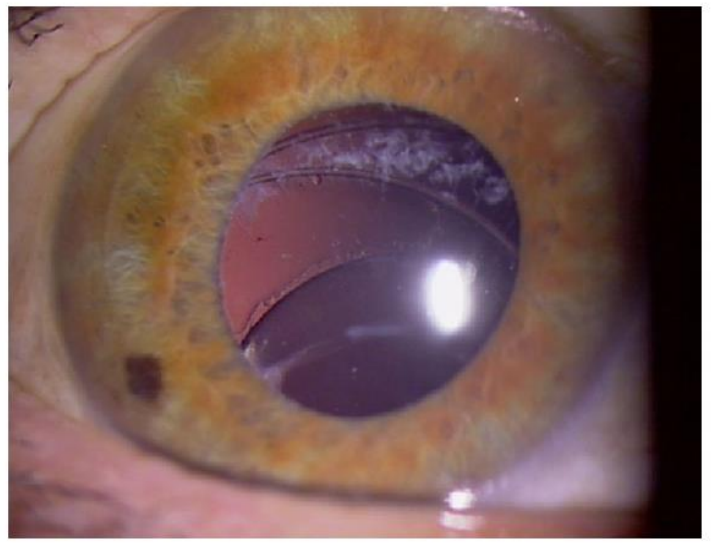

(a)

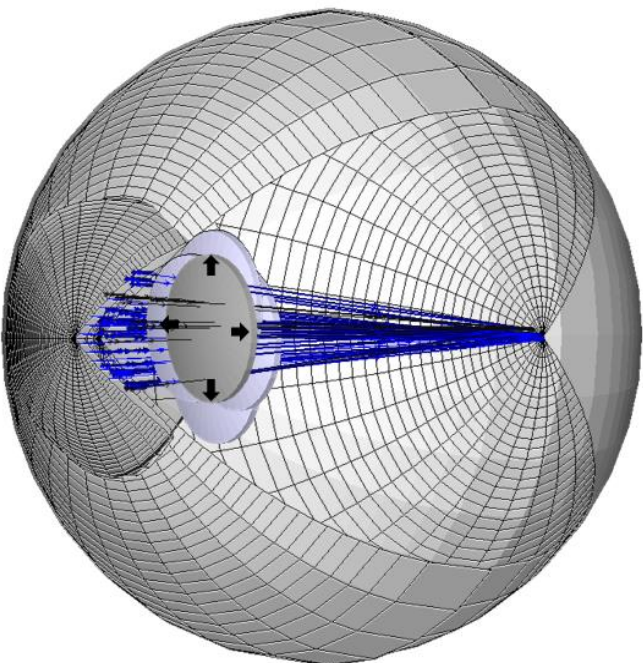

(b)

Figure 2.22 (a) Real IOL decentration in patient's eye after cataract surgery (b) solid model $3 / 4$ draw sections of customized eye model with simulated decentrations ranging is from $-1-1 \mathrm{~mm}$ in $0.1 \mathrm{~mm}$ steps for $5 \mathrm{~mm}$ pupil diameter along horizontal and vertical meridians, with reference to the pupil center 


\subsubsection{MATLAB routines}

MATLAB is a powerful numerical computing package with a large range of mathematics and visualization tools, which is useful for post-processing Zemax simulations. As we stated in Section 2.1.2 (Quantification tools of 3D anterior segment spectral domain OCT), in order to produce automated image processing and analysis techniques that can overcome the above mentioned limitations, a series of custom algorithms have been developed in the VioBio lab before, which included algorithms for inputting data, denoising of raw data, statistical thresholding, clustering, 3D merging, multilayer segmentation, pupil centering, geometrical distance calculation, distortion corrections and surface fitting by Zernike terms and quadrics (Ortiz, Siedlecki et al. 2010). Recently Eduardo et al. (E. Martínez-Enriquez 2016) improved the customized algorithm of quantitative anterior segment OCT and developed dedicated algorithms to estimate lens volume (VOL), equatorial diameter (DIA) and equatorial plane position (EPP) (shown in Figure 2.24).

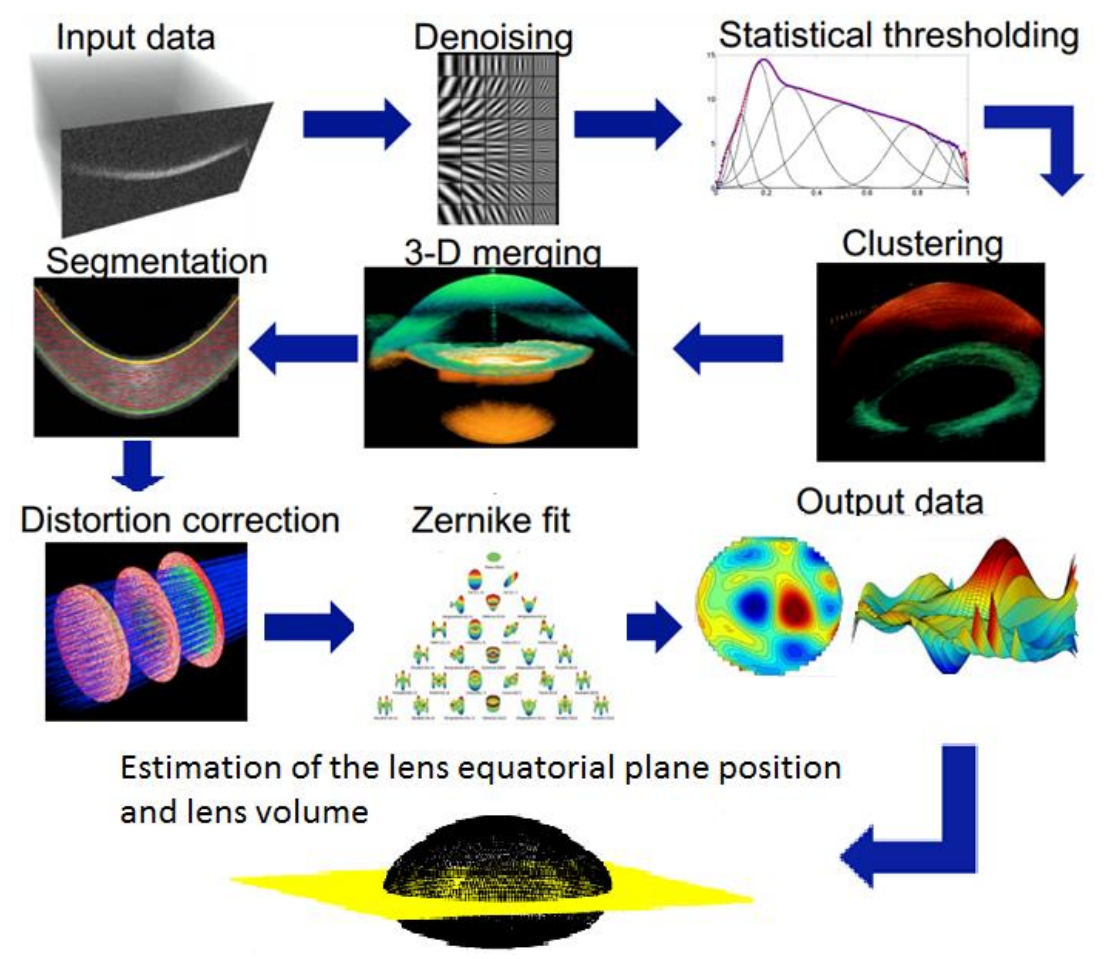

Figure 2.23 Illustration of OCT automatic image processing (Ortiz, Siedlecki et al. 2010, E. Martínez-Enriquez 2016)

The MATLAB software program also took exported data from the instrument and created a suitable file for input into the Zemax program when corneal topography measurements were performed. The .DAT file generated by the MATLAB function was imported to Zemax and used to define a Zernike sag surface. The elevation data of the corneal was fit with a Zernike polynomial expansion using 28 terms. Since Zemax order and OSA order are different, Algorithm 1 allows switching between the orders: 


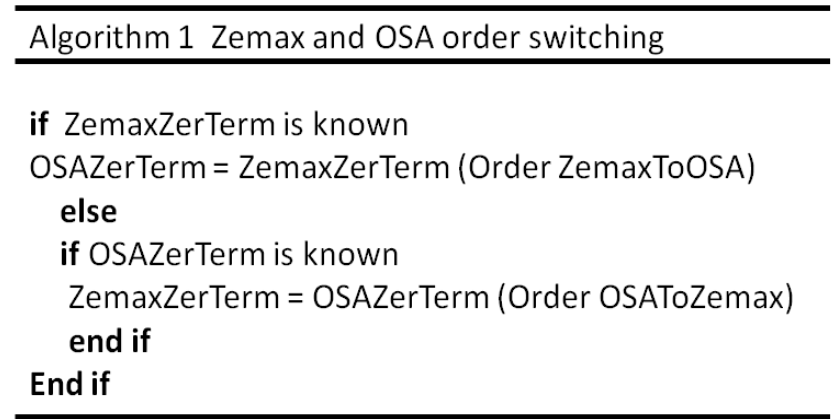

These .DAT files are imported into Zemax when creating personalized pseudophakic eye models (see Chapter 5) to define the anterior and posterior corneal surface by Algorithm 2.

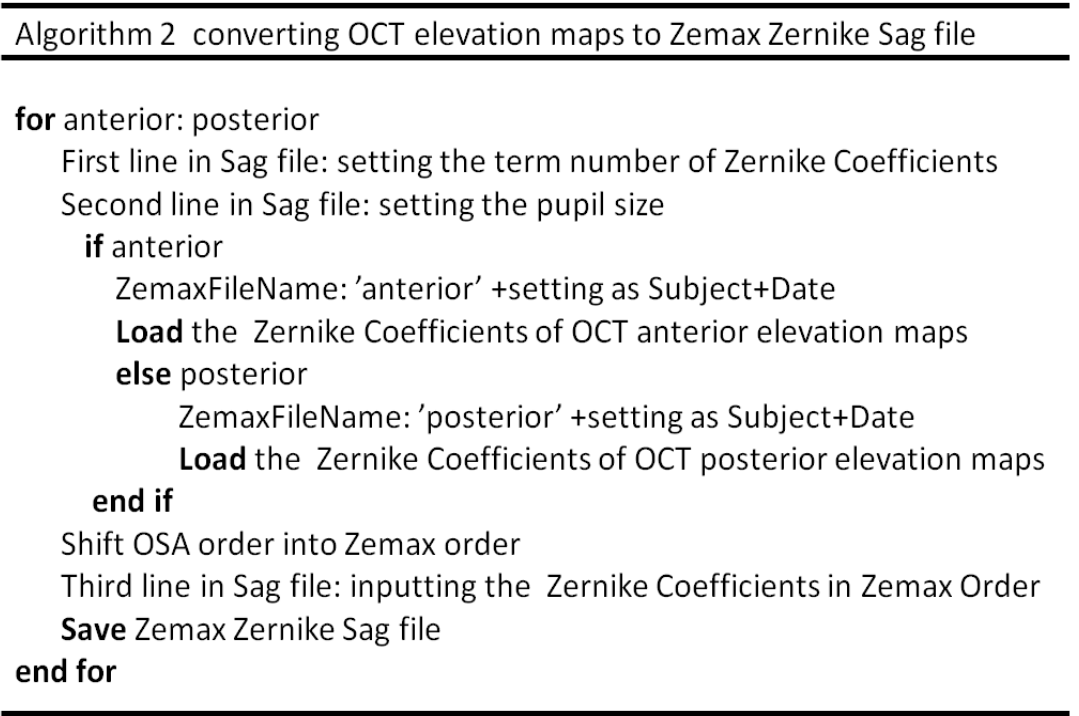

In order to analyze 3D-OCT surface topography of isolated human crystalline lenses, Algorithm 3, 4 and 5 were written in MATLAB which allowed for the evaluation of surface elevation with higher order terms, their relationships between anterior and posterior lens surfaces, and potential changes with age, similar to previous analysis on corneal surface topographies. To ensure optimal signal from both lens surfaces, each set of 3D images consisted of two acquisitions at two different planes of focus which were merged to produce a complete image of anterior surface, posterior surface, and the cuvette holding the lens, using customized MATLAB programs. The images were merged using the cuvette as a reference to produce a full 3D image of the crystalline lens. Custom algorithms merged the images of the crystalline lens, obtaining images of both lens surfaces in two different planes and the cuvette distorted by the lens, fan distortion and optical distortion (de Castro, Ortiz et al. 2010), obtaining topographic lens surface elevation maps and lens alignment by steepest meridian of the anterior surface (shown in Figure 2.25). 
Chapter 2

Experimental methods, computational techniques and other types of validation

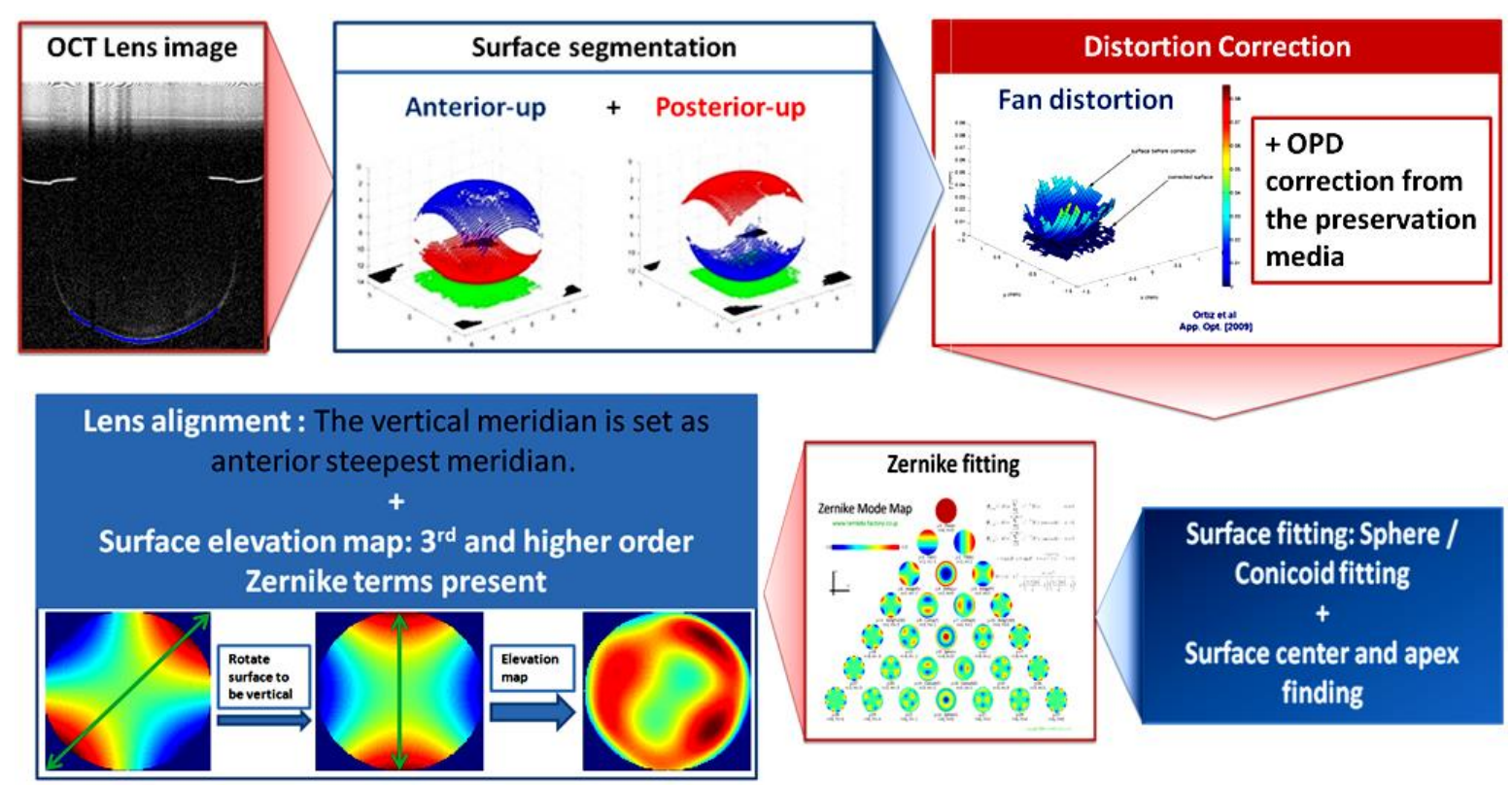

Figure 2.24 Illustrated algorithm of OCT 3D surface topography of isolated human crystalline lenses

Algorithm 3 Astigmatism magnitude and angle calculation of anterior surface

Load ZernikeCoe_anterior surface

Calculation of Astigmatism power at $45 \mathrm{deg}$ axis. Unit is diopter.

$J_{45}$ antAst. $=\frac{-2 \times \sqrt{6} \times \operatorname{ZerCoe}(4)}{(\text { Pupil_radius })^{2}}$

Calculation of Astigmatism power at 0 deg or $180 \mathrm{deg}$ axis. Unit is diopter.

$J_{180 \_ \text {ant Ast. }}=\frac{-2 \times \sqrt{6} \times \operatorname{Zer} \operatorname{Coe}(6)}{(\text { Pupil_radius })^{2}}$

Calculation of magnitude of surface Astigmatism.

magnitude_antAst. $=-2 \times \sqrt{J_{45}^{2}+J_{180}^{2}}$

Calculation of angle of surface Astigmatism. Unit is radian.

Reference axis is horizontal axis (0 deg axis).

$\alpha_{-}$antAst. $=\tan ^{-1}\left(\frac{J_{45}}{J_{180}}\right) / 2$

Algorithm 4 rotating posterior surface with anterior Astigmatism angle:

Load posterior surface data $\left(X_{p}, Y_{p}, Z_{p}\right)$

Load anterior Astigmatism angle $\alpha$ antAst.

Transfer radian angle to degree angle:

antAstAng $=\frac{\alpha_{-} \text {ant Ast. } \times 180}{\pi}$

Coordinate of anterior and posterior Crystalline lens surfaces are mirror symmetric.

Rotate posterior surface with anterior Astigmatism angle counterclockwise:

$$
\begin{aligned}
& X_{p}^{\prime}=X_{p} \times \cos (- \text { antAstAng })-Y_{p} \times \sin (- \text { antAstAng }) \\
& Y_{p}^{\prime}=Y_{p} \times \cos (- \text { antAstAng })+X_{p} \times \sin (- \text { antAstAng })
\end{aligned}
$$

Save rotated posterior surface data $\left(X_{p}^{\prime}, Y_{p}^{\prime}, Z_{p}\right)$ 


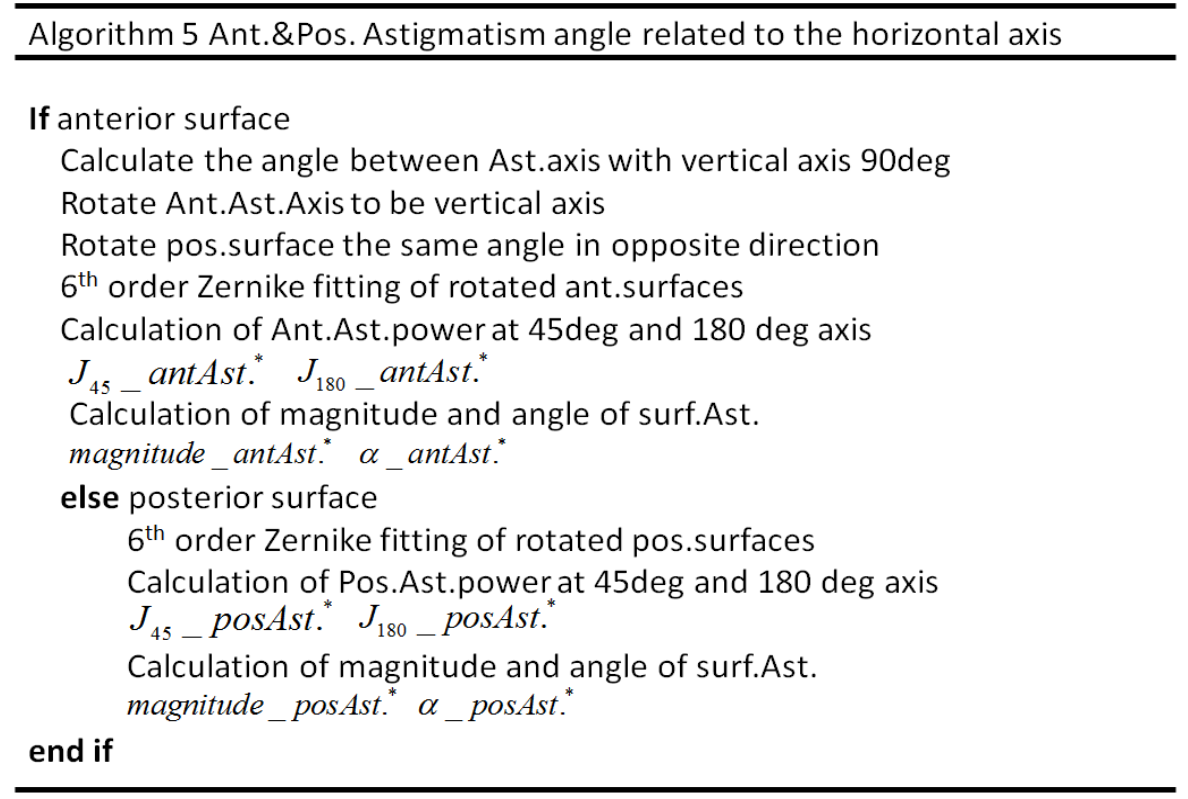

As the step of pupil centering in OCT image processing, the apex of the lens was used as a reference to center each surface. Lens surface elevation maps (obtained by subtraction of the best fitting spheres from the segmented surfaces) were fitted to $6^{\text {th }}$ order Zernike polynomials with 28 terms in a $6 \mathrm{~mm}$ pupil diameter. Descriptive parameters of the surface elevation maps include individual surface Zernike coefficients (up to $6^{\text {th }}$ order), and the RMS of combination of some terms (RMS astigmatism, RMS trefoil, RMS tetrafoil, RMS spherical terms, RMS coma and RMS high order Astigmatism) for statistical analysis.

Dynamic Data exchange (DDE) is a method for MATLAB and Zemax to pass information to each other. Taking advantage of the MATLAB mathematical and plotting libraries to analyze Zemax is more effective and automatic. IOLs from the catalog could be implanted into the computer eye model by updating Zemax editors with contents of server memory, and also could change the IOL position from MATLAB (Algorithm 6 and 7). The Zemax simulation is accessible to get the optical quality at the image surface as retina of human eye by ray tracing, and MATLAB commands allow getting Zernike coefficients from Zemax aberration report directly and saving those values into excel. 


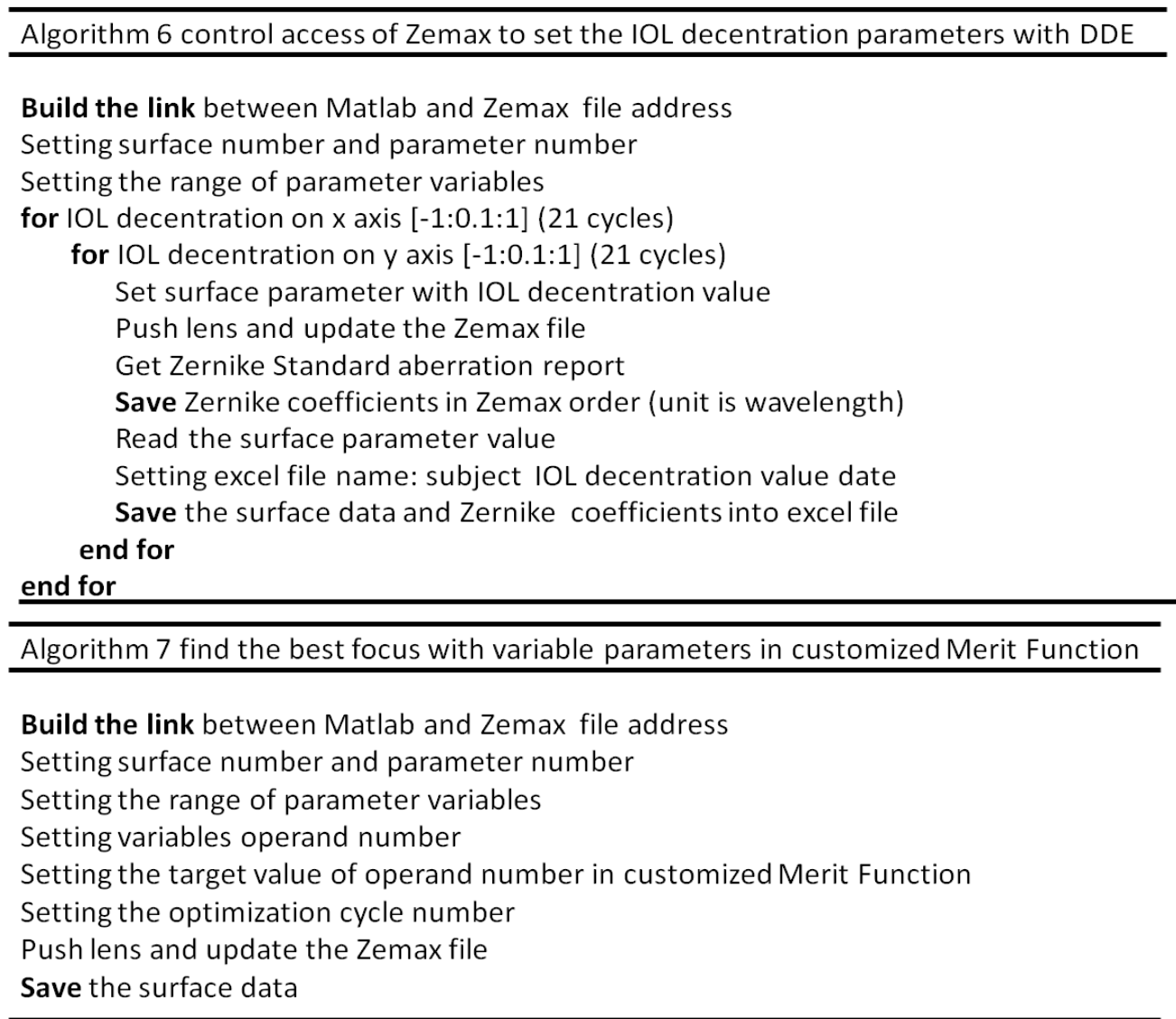

The PSF, MTF and OTF were computed using Fourier optics from Zernike coefficients using routines written in MATLAB. The PSF for the corresponding levels of defocus was generated using custom-developed routines written in MATLAB for 5-mm pupils. The defocus term was set to 0 , and to the value corresponding to the defocus setting shift (1.5D to $-1.5 \mathrm{D}$ with $0.125 \mathrm{D}$ as step) for any other condition. Optical quality was described in terms of the Visual Strehl metric. Visual Strehl was computed as the volume under the Visual MTF normalized to the diffraction limit. The VS was evaluated through focus (considering HOAs with/without the astigmatic terms). The maximum value of the through-focus Visual Strehl curve was obtained as the best corrected optical quality metric. A Customized algorithm created in MATLAB calculated the VS across IOL decentrations in the range of $2 \mathrm{~mm}$ with 0.1 as step, plot a table chart with a $12 \times 12$ square symbols to present VS value of simulation coded by color (red means high value, and blue mean low value). Based on the highest VS location, the optimal IOL position was defined. 


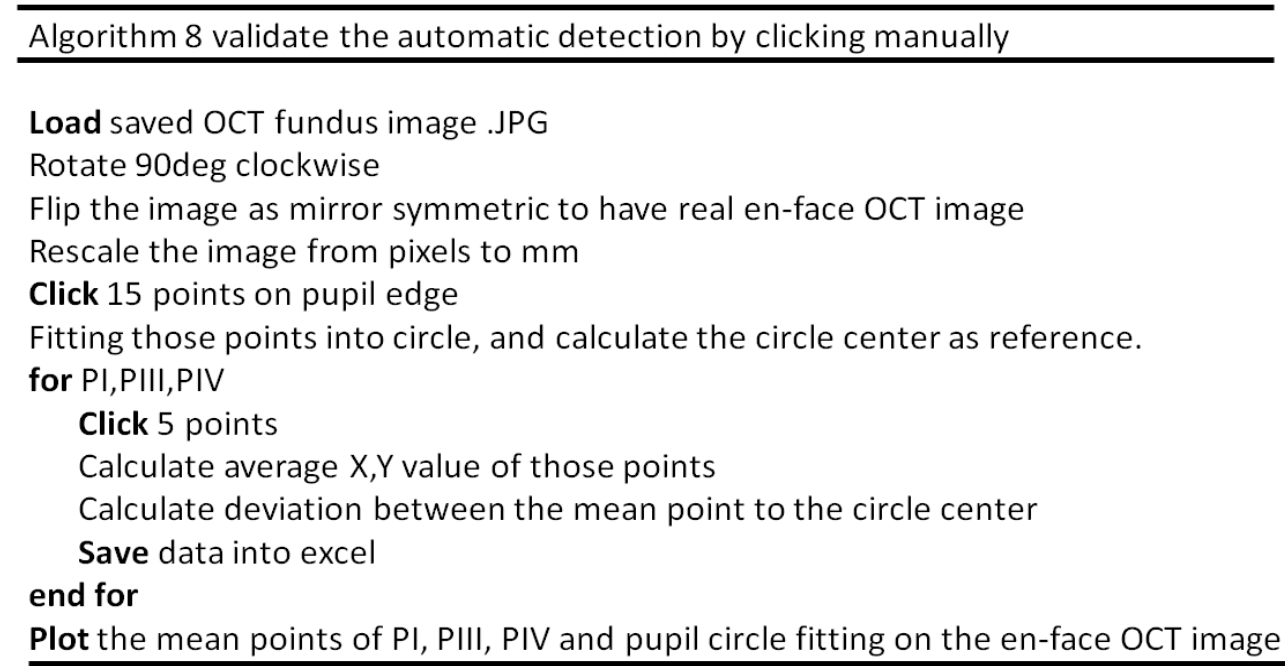

As we maintained in Section 2.1.3, the detection of Purkinje images PI, PIII and PIV and pupil center in the pupillary images is performed with a customized algorithm using MATLAB. It also allowed generation of image intensity values in the vertical axial direction, to distinction of pupil edge for pupil segmentation and center finding by ellipse fitting, the results of which were validated by clicking 15 points on the pupil edge manually, then the positions of the specular reflexes in the different surfaces were marked by clicking 5 points manually (Purkinje-like images positions, PI, PIII, and PIV) and horizontal and vertical position with reference to the center of the pupil were saved automatically. 


\section{CHAPTER 3 \\ INTRAOCULAR LENS \\ ALIGNMENT FROM AN EN- \\ FACE OCT IMAGE \\ PURKINJE-LIKE METHOD}


This chapter is based on the following publication:

M. Sun, A. de Castro, S. Ortiz, P. Perez-Merino, J. Birkenfeld and S. Marcos (2014). "Intraocular lens alignment from an en-face optical coherence tomography image Purkinje-like method." Optical Engineering 53(6): 061704-061704.

The contribution of Mengchan Sun to the study, in collaboration with other coauthors, was the literature search, the design of the experiments and the data acquisition (in collaboration with Alberto de Castro), the development of specific routines (in collaboration with Sergio Ortiz and Alberto de Castro), the simulations of Purkinje image and the analysis of the data. 


\section{RESUMEN}

Objetivos: El ojo humano es un sistema óptico no centrado, con la lente generalmente inclinada y descentrada con respecto a otros ejes en el ojo. El desalineamiento de las lentes intraoculares (IOL) implantadas en la cirugía de cataratas es particularmente relevante, ya que puede tener un impacto en el rendimiento óptico de nuevos diseños de IOLs más sofisticados. El capítulo presenta un nuevo método para medir la inclinación y la descentración del cristalino on IOL basado en las posiciones de las llamadas imágenes de Purkinje (reflexiones desde la superficie corneal anterior y superficies de las lentes anterior y posterior), linealmente relacionadas con la IOL Inclinación y descentración. En el método presentado se hace uso de imágenes OCT" en-face", que muestran reflejos de Purkinje similares, pero que, debido a la naturaleza tomográfica de la OCT, pueden ser inequívocamente identificados y no enmascarados por potenciales superposiciones.

Métodos: La medición de lentes intraoculares (IOL) implantada en pacientes en cirugía de catarata es importante para entender su rendimiento óptico. Presentamos un método para estimar la inclinación y descentralización de las IOL a partir de imágenes de Tomografía de Coherencia Óptica (OCT). Las imágenes en-face OCT muestran imágenes similares a Purkinje que corresponden a las reflexiones especulares de las superficies corneal y de IOL. A diferencia de la imagen de Purkinje estándar, la naturaleza tomográfica de OCT permite una asociación inequívoca de la reflexión con la superficie correspondiente. Las localizaciones del tipo Purkinje son combinaciones lineales de inclinación de IOL, descentración de IOL y rotación de ojo. Los coeficientes de ponderación dependen de la geometría del segmento anterior individual, obtenida a partir del mismo conjunto de datos de OCT. La metodología se demuestra en un ojo modelo artificial con cantidades fijadas de la inclinación y de la descentración de la lente, y en 5 ojos pseudofáquicos.

Resultados: La inclinación y descentración medidas en el ojo artificial difirieron en 3.7\% y $0.9 \%$, respectivamente, de los valores nominales. En pacientes, la inclinación media de la IOL y la descentralización de Purkinje fueron de $3.30 \pm 4.68$ grados y $0.16 \pm 0.16 \mathrm{~mm}$, respectivamente, y difirieron en promedio de 0.5 grados y $0.09 \mathrm{~mm}$, respectivamente, de las mediciones directas en las imágenes OCT corregidas por distorsión.

Conclusiones: Junto con la biometría / geometría ocular, la alineación de la IOL se puede incorporar en modelos de ojo computacionales personalizados para evaluar los factores que contribuyen a la degradación óptica en pacientes implantados con IOL. La metodología basada en Purkinje a partir de imágenes OCT en-face en el segmento anterior proporcionó, por tanto, medidas fiables de la inclinación y descentración de la IOL. 


\subsection{Introduction}

Cataracts are one of the major causes of vision loss in the aging population. The common treatment for cataracts is the replacement of the natural lens by an intraocular lens (IOL). In an intracapsular procedure, the IOL is inserted in the capsular bag, following phacoemulsification and aspiration of the crystalline lens material, through a circular window in the anterior capsule (capsulorhexis), with the lens haptics pressing against the equatorial region of the capsular bag. The stability of the IOL in the capsular bag depends on the material and design of the haptics. With state-of-the art monofocal IOLs, IOL tilt and decentrations are generally of similar amounts to those of the natural crystalline lens (de Castro, Rosales et al. 2007).

Originally, the main goal of a cataract treatment was to eliminate scattering, but IOLs have evolved enormously, first to additionally correct for refractive errors, but more recently, to compensate for HOA, particularly spherical aberration. Also, new IOL designs aim at correcting presbyopia, either by expanding the depth-of-focus of the eye (multifocal IOLs, M-IOLs) or by dynamically changing the refractive power (accommodating IOLs, AIOLs). With the increased sophistication of IOL designs, the impact of the IOL position becomes more critical. The aimed correction of corneal HOA by aspheric IOLs may be compromised by the introduction of other HOA by a tilted/decentered lens(Atchison 1991). The performance of refractive M-IOLs (generally with concentric or segmented near/far regions) is critically affected by misalignments of the IOL (Menapace, Findl et al. 2007). Finally, tilts and decentrations occurring with the only FDA-approved A-IOL may also play a role in its function(Marcos, Ortiz et al. 2013). Measurement of tilt and decentration of IOLs in pseudophakic eyes is therefore important as an endpoint for treatment, particularly with premium IOLs. On the other hand, in combination with biometric and anatomic ocular data, knowledge of IOL and eye alignment is important in the development of customized computer eye models, which give insights into the relative contribution of different factors of optical performance with IOLs (Rosales and Marcos 2007, Rosales, Wendt et al. 2008). Several methods have been presented to measure IOL tilt and decentration as maintained in Section 1.4.1. Purkinje imaging relies on the acquisition of pupillary images containing Purkinje images I, III and IV. The relative positions of PI, PIII and PIV with respect to the pupil center are proportional to eye rotation, IOL tilt and IOL decentration. The Purkinje imaging device is simple and the measurement typically robust, although it may be subject to some limitations: difficulties in the association of the images to the corresponding reflecting surfaces (some authors have solved this by projecting a semicircular ring of LEDs, yielding relative inverted images for PI and PIV (Tabernero, Benito et al. 2006)); overlapping of the different Purkinje images (usually overcome by eccentric illumination and image acquisitions at different eccentric fixations) (Rosales and Marcos 2006); requirement of ocular geometrical and biometric information (normally obtained from other instruments).

As maintained in Section 1.4.1, Scheimpflug imaging has also been used to estimate tilt and decentration of IOLs by analysis of the orientation and shift of the lens in crosssectional images of the anterior segment of the eye (Coppens, van den Berg et al. 2005, de Castro, Rosales et al. 2007). The 3D tilt and decentration can be obtained from sinusoidal fitting of the 2D coordinates. While the method is direct (i.e. relies on the direct viewing of 
the lens), it is subject to several limitations: refraction distortion of the lens images and relatively sparse meridional sampling. IOL tilt and (to a less extent) decentration from this technique are in good agreement with Purkinje-based measurements (de Castro, Rosales et al. 2007). The accuracy and precision of the Scheimpflug data were tested in measurements in an artificial eye, with a discrepancy from nominal values of $0.24 \mathrm{deg}$, on average, for tilt ( $0.28 \mathrm{deg}$ for Purkinje-based tilt), and $0.23 \mathrm{~mm}$, on average, for decentration $(0.01 \mathrm{~mm}$ for Purkinje-based decentration).

3D anterior segment spectral domain OCT provides accurate anterior and posterior corneal topography and pachymetry (Ortiz, Siedlecki et al. 2010, Ortiz, Siedlecki et al. 2011), anterior and posterior lens topography (Ortiz, Perez-Merino et al. 2012), pupilometry, and anterior chamber depth (Ortiz, Perez-Merino et al. 2013) as maintained in Section 2.1.2. In addition, the direct view of the lens also allowed estimates of tilt and decentration ( $\mathrm{Li}$, Wang et al. 2013, Marcos, Ortiz et al. 2013, Ortiz, Perez-Merino et al. 2013, Wang, Dong et al. 2013).

In this chapter, we developed Purkinje-based methodology from anterior segment en-face OCT images. The technology resembles the Purkinje method, since three spots are observed in the en-face OCT images. These spots originate from the reflections of light on the anterior surface of the cornea and the anterior and posterior surfaces of the lens, although, in contrast to the standard Purkinje method, the spots are not formed by the entire area of the ocular surfaces. Instead, the OCT system collects the scattered light reflected back from the tissue and, when the reflected ray direction coincides with the OCT axis, a strong specular reflection is captured in the test arm of the OCT, producing a bright spot in the enface OCT images. Unlike in the standard Purkinje method, which relies on images in a single plane of focus, the tomographic information of the OCT images relates each reflection with the corresponding surface, as well as avoiding image overlapping.

The Purkinje image analysis (estimation of coefficients in a set of linear equations) requires assumption of an eye model. Assumption of a general computer eye model results in a decrease in the accuracy of the tilt and decentration estimates (Rosales and Marcos 2006). Instead, individual anatomical and biometric parameters are used, generally obtained from corneal topography or keratometry, optical biometry and Purkinje phakometry. These parameters can be retrieved from the same OCT data set (Ortiz, Perez-Merino et al. 2012, Ortiz, Perez-Merino et al. 2013). In Section 2.1.3, we introduced the measurement of IOL tilt and decentrations from OCT images, using a Purkinje-based analysis. The method was validated on an artificial model eye (with set values of lens tilt and decentration) and tested in 5 pseudophakic eyes with implanted A-IOLs (where the en-face OCT Purkinje-based estimates of IOL tilt and decentration were compared to direct estimates from distortioncorrected 3D OCT images) in this chapter. 


\subsection{Materials and Methods}

\subsubsection{Laboratory-Based SD-OCT System}

The SD-OCT system used in the measurements has been described in detail in Section 2.1. The lateral sampling resolution ranged between 0.04 and $0.2 \mathrm{~mm}$. Dedicated image processing routines allowed segmentation of the optical surfaces and quantification of their geometry with distortion correction as maintained in Section 2.1.2 (Quantification tools).

\subsubsection{Artificial eye}

Detailed information of the water-cell physical model eye has been described in Section 2.3.3. The geometry of the A-IOL was characterized using microscopy-based non-contact optical profilometry (Sensofar, PL $\mu 2300$, Barcelona, Spain), introduced before in Section 2.3.2. The profiles were fit with conics and potential tilts of the IOL were removed using custom routines written in MATLAB (Mathworks, USA). The measurements were repeated 5 times and the mean values are shown in Section 2.3, Table 2.1.

The refractive index of the cornea at the OCT wavelength was calculated by dividing the central optical path length obtained from the OCT images by the nominal geometrical central thickness $(n=1.486$, group refractive index at $840 \mathrm{~nm})$. The thickness of the IOL (CrystalensAO 23.5D) was measured using the profilometry system, and its refractive index was also calculated from the OCT images $(n=1.427$, group refractive index at 840 $\mathrm{nm})$. Measurements were obtained for the artificial eye with the lens in air (i.e. cell not filled with water) and in water. The IOL was tilted inside the water-cell eye from -5 deg to $5 \mathrm{deg}$ in 1-deg steps and decentered from -1.9 to $1.9 \mathrm{~mm}$ in $0.6 \mathrm{~mm}$-steps.

\subsubsection{Patients}

Images were collected in 5 eyes of 4 patients ( $74 \pm 2.3$ years old) 90 days after IOL implantation (Crystalens A-IOL, Bausch and Lomb). Biometric data on these patients has been previously reported as part of a larger study aimed at characterizing performance of these IOLs (Marcos, Ortiz et al. 2013). Only patients implanted with 23.5-D power IOLs were selected (as this IOL was available for in vitro measurements). As we maintained in Section 2.5.2, measurements in post-operative patients were performed under mydriasis (phenylephrine 10\%) with fixation on a reference E-letter target projected on a minidisplay at optical infinity.

\subsubsection{OCT image processing}

The image processing algorithm for geometrical characterization of the OCT images, described in detail in Section 2.1.2 (Quantification tools) is comprised of: image denoising; statistical thresholding; volume clustering; multilayer automatic segmentation; pupil center registration; 3D volume merging of the cornea and IOL images, obtained in two different acquisitions; geometrical distances calculation; fan distortion correction; optical distortion correction; surface fitting to spherical surfaces. In this study, a 3-mm diameter optical zone is used with respect to the corresponding surface apex. Anterior segment biometry, including central corneal thickness (CCT), anterior chamber depth (ACD), intraocular lens 
thickness (ILT), intraocular lens position (ILP), and IOL tilt (IT) were computed from the corrected 3D OCT images. The refractive indices used in the calculations are reported in Table 3.1.

Table 3.1 Radii of curvature, axial biometry, and refractive indices, in the physical model eye, and the five patient eyes.

\begin{tabular}{|c|c|c|c|c|c|c|}
\hline Eye \# & Artificial eye model & S\#3-OD & $\mathrm{S} \# 8-\mathrm{OD}$ & S\#10-OD & S\#4-OS & S\#8-OS \\
\hline Anterior corneal radius of curvature $(\mathrm{mm})$ & 7.800 & 7.592 & 7.662 & 7.448 & 7.297 & 7.706 \\
\hline Posterior corneal radius of curvature $(\mathrm{mm})$ & 6.480 & 6.217 & 6.825 & 6.305 & 6.155 & 6.842 \\
\hline Anterior intraocular lens $(\mathrm{IOL})$ radius of curvature $(\mathrm{mm})$ & 7.781 & 7.781 & 7.781 & 7.781 & 7.781 & 7.781 \\
\hline Asphericity $\mathrm{Q}$ of anterior IOL radius & -1.35 & -1.35 & -1.35 & -1.35 & -1.35 & -1.35 \\
\hline Posterior IOL radius of curvature $(\mathrm{mm})$ & -7.937 & -7.937 & -7.937 & -7.937 & -7.937 & -7.937 \\
\hline Asphericity $\mathrm{Q}$ of posterior $\mathrm{IOL}$ radius & -2.356 & -2.356 & -2.356 & -2.356 & -2.356 & -2.356 \\
\hline Corneal thickness & 0.500 & 0.581 & 0.501 & 0.587 & 0.581 & 0.482 \\
\hline Anterior chamber depth $\mathrm{ACD}(\mathrm{mm})$ & $3.825^{\mathrm{a}} / 4.124^{\mathrm{b}}$ & 3.413 & 3.444 & 3.276 & 3.712 & 3.136 \\
\hline IOL thickness $(\mathrm{mm})$ & 1.100 & 1.100 & 1.100 & 1.100 & 1.100 & 1.100 \\
\hline Corneal refractive index & 1.450 & 1.396 & 1.400 & 1.312 & 1.396 & 1.400 \\
\hline Aqueous humor refractive index & 1.346 & 1.346 & 1.346 & 1.346 & 1.346 & 1.346 \\
\hline IOL refractive index & 1.440 & 1.440 & 1.440 & 1.440 & 1.440 & 1.440 \\
\hline Vitreous humor refractive index & 1.346 & 1.346 & 1.346 & 1.346 & 1.346 & 1.346 \\
\hline
\end{tabular}

aEye model in air.

'Eye model in water.

\subsubsection{Purkinje-like OCT method}

In Section 2.1.3, we described the measurement of lens tilt and decentration by Purkinjelike OCT en-face reflection imaging. As a brief summary, the Purkinje-like analysis can be summarized in five steps: collection of a volumetric data set; generation of image intensity values in the axial direction; pupil segmentation and identification of the positions of the specular reflexes on the different surfaces (Purkinje-like images positions: PI, PIII and PIV). The reflexes PI, PIII, and PIV are easily identified by checking the corresponding OCT B-scan image. Eye rotation $(\beta)$, lens tilt $(\alpha)$ and decentration $(d)$ were obtained from Eq.2.4, using the positions of the experimental reflexes (PI, PIII, and PIV). 


\subsection{Results}

\subsubsection{Validation on a water-cell physical eye model}

The developed methodology was tested on the physical eye model described before. The corresponding coefficients of Eq.2.4 were computed for the artificial eye (see Table 3.2.5.1 for both the model eye in air, and immersed in water).

Table 3.2 A-G coefficients in Eq.2.4for the physical model eye in air and water and the 5 eyes of the study

\begin{tabular}{lccccccc}
\hline \hline & $\mathrm{A}$ & $\mathrm{B}$ & $\mathrm{C}$ & $\mathrm{D}$ & $\mathrm{E}$ & $\mathrm{F}$ & $\mathrm{G}$ \\
\hline Artificial eye model $^{\mathrm{a}}$ & $0.112-0.248$ & -0.074 to -0.120 & 0.899 to 1.830 & 0.968 to 1.028 & 0.130 to 0.143 & 0.192 to 0.336 & -0.017 to 0.020 \\
S\#3 OD & 0.213 & -0.108 & 1.644 & 0.937 & 0.064 & 0.187 & -0.094 \\
S\#8 OD & 0.213 & -0.108 & 1.645 & 0.936 & 0.066 & 0.187 & -0.093 \\
S\#10 OD & 0.220 & -0.107 & 1.688 & 0.924 & 0.064 & 0.192 & -0.092 \\
S\#4 OS & 0.224 & -0.109 & 1.720 & 0.943 & 0.054 & 0.200 & -0.094 \\
S\#8 OS & 0.208 & -0.107 & 1.606 & 0.926 & 0.073 & 0.179 & -0.091 \\
\hline \hline
\end{tabular}

* Range corresponding to different realizations (air and water) of the model eye

Figure 3.1 shows the experimental tilt and decentration measured in the physical model eye, against the nominal set values of tilt and decentration, for the IOL in air $(a, c)$ and water $(b, d)$. There is a high correspondence between the experimental and nominal values: slope $=1.007 ; \mathrm{r}=0.9902, \mathrm{p}<0.0001$ for IOL tilt in air; slope $=1.037 ; \mathrm{r}=0.9973, \mathrm{p}<0.0001$ for IOL tilt in water; slope $=0.991 ; \mathrm{r}=0.9878 ; \mathrm{p}<0.0001$ for IOL decentration in air; slope $=0.993$; $\mathrm{r}=0.9993 ; \mathrm{p}<0.0001$ for IOL decentration in water. The average difference between nominal and experimental value was $0.254 \pm 0.170 \mathrm{deg}$ for tilt, and $0.066 \pm 0.017 \mathrm{~mm}$ for decentration.

\subsubsection{IOL tilt and decentration in patients}

Measurements were performed on 5 eyes of 4 patients, all implanted with a $23.5 \mathrm{D}$ Crystalens A-IOL. The biometric parameters necessary to build the computer eye model were obtained from OCT (anterior and posterior corneal radius, corneal thickness, ACD) (Marcos, Ortiz et al. 2013) and from non-contact profilometry (anterior and posterior IOL radii of curvature and thickness). Table 3.1 shows the corresponding parameters for all 5 eyes. The two sets of coefficients for tilt and decentration correspond to two different realizations of the artificial eye model (the anterior chamber depth was enlarged for IOL tilt measurements to allow proper positioning of the holding platform inside the chamber). 


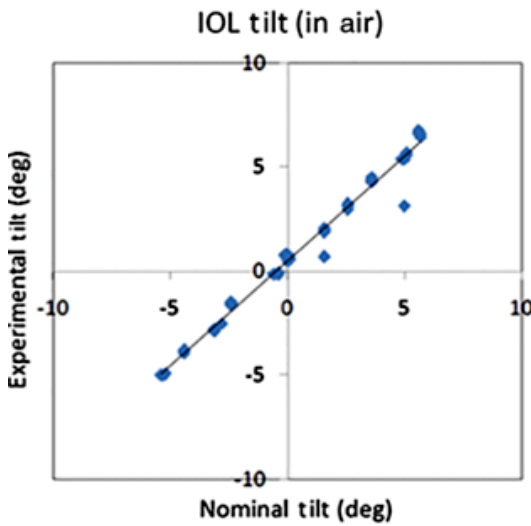

(a)

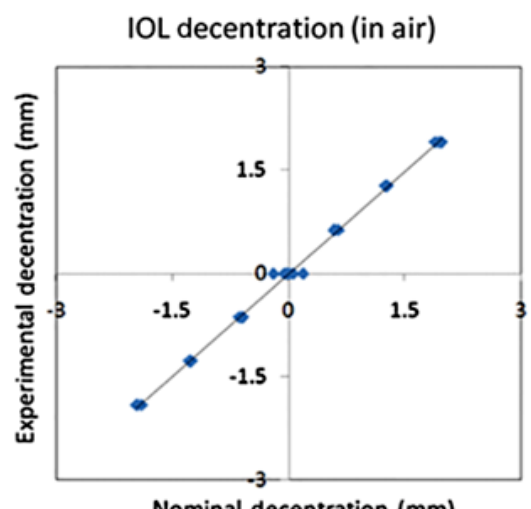

(c)

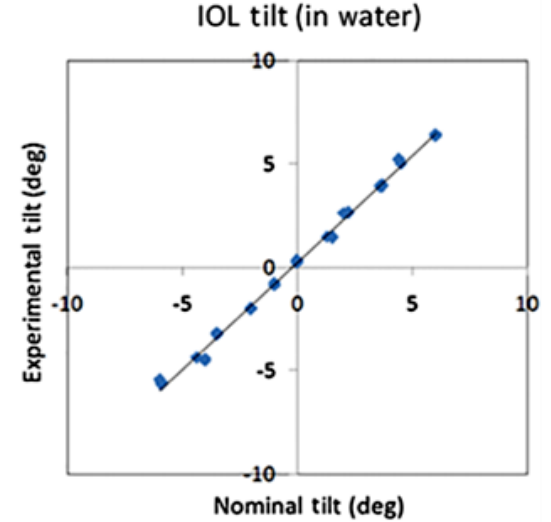

(b)

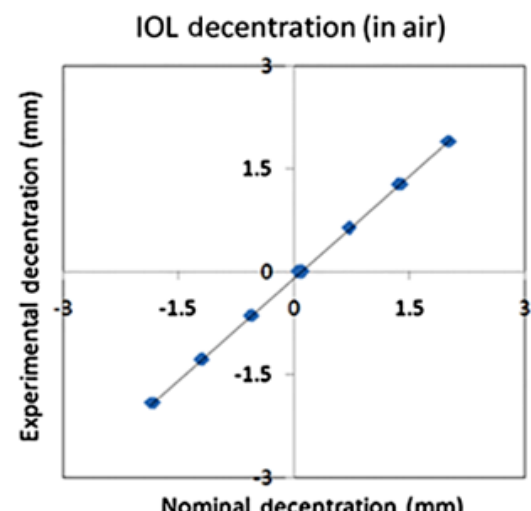

(d)

Figure 3.1 Experimental data vs. nominal data in the artificial eye model (a) IOL tilt (in air); (b) IOL tilt (in water); (c) IOL decentration (in air); (d) IOL decentration (in water)

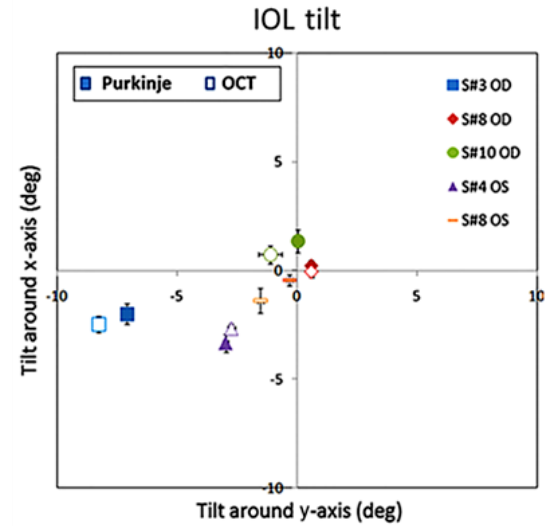

(a)

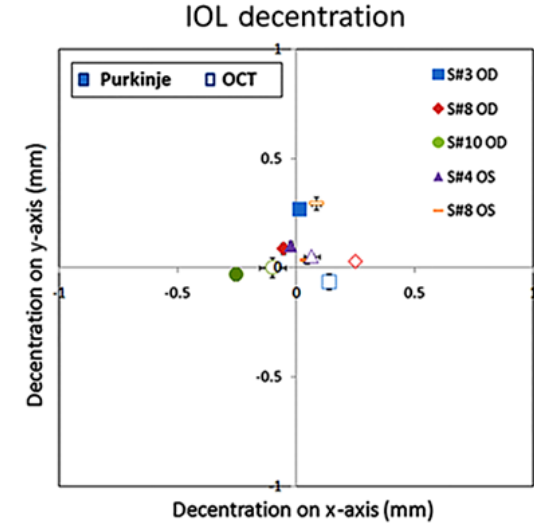

(b)

Figure 3.2 (a) IOL tilt and (b) IOL decentration in the 5 eyes studied. Solid symbols represent data obtained using the Purkinje-like OCT-based method and open symbols represent data obtained directly from distortion-corrected 3D OCT images (Ortiz, Perez-Merino et al. 2013). Square, rhombus and round represent right eyes (OD) while triangle and rectangle represent left eyes (OS). Each color (color online only) represents a different eye. Error bars stand for standard deviations of repeated measurements.

IOL tilt is defined as the angle between the IOL axis and the pupillary axis, where the IOL axis is the line joining the centers of curvature of the IOL, and the pupillary axis joining 
the center of curvature of the cornea and the pupil center. IOL decentration is defined as the distance between the IOL center and the pupil center. According to existing conventions(Rosales, Wendt et al. 2008): positive horizontal decentration stands for nasal decentration (in right eyes) and temporal decentration (in left eyes), and vice versa for negative; and positive vertical decentration for superior, and negative for inferior. Positive tilt about the horizontal axis (Tilt $\mathrm{x}$ ) indicates that the superior edge of the lens is moved forward. Negative tilt around the vertical axis (Tilt y), in right eyes, indicates that the temporal edge of the lens is moved forward, and, in left eyes, indicates that the nasal edge of the lens is moved forward.

We did not find consistent mirror symmetry in the orientation of tilt or decentration in these eyes (Figure 3.2). The average tilt about the $\mathrm{x}$-axis was -0.14 deg in right eyes and -1.88 deg in left eyes (Purkinje-like), and -0.59 deg in right eyes and -2.02 deg in left eyes (OCT); Average tilt about the y-axis was $2.15 \mathrm{deg}$ in right eyes and $1.65 \mathrm{deg}$ in left eyes (Purkinjelike) and $2.93 \mathrm{deg}$ in right eye and $2.14 \mathrm{deg}$ in left eyes (OCT). Average decentration on the horizontal axis was $-0.10 \mathrm{~mm}$ in right eyes and $0.00 \mathrm{~mm}$ in left eyes (Purkinje-like), and $-0.10 \mathrm{~mm}$ in right eyes and $0.11 \mathrm{~mm}$ in left eyes (OCT); Average decentration on the vertical axis was $-0.08 \mathrm{~mm}$. The average standard deviations for repeated measurements of tilt were $0.47 \mathrm{deg}$ (Purkinje-like) and $0.58 \mathrm{deg}$ (OCT). The standard deviation for repeated measurements of decentration was $0.04 \mathrm{~mm}$ (Purkinje-like) and $0.06 \mathrm{~mm}$ (OCT).

Figure 3.3 compares the coordinates of tilt $(a, b)$ and decentration $(c, d)$ measured with both techniques. There was a high correlation between both estimates, particularly for tilt (slope: $1.18, \mathrm{r}=0.95, \mathrm{p}<0.012$, for tilt around $\mathrm{x}$-axis; slope: $0.92, \mathrm{r}=0.98, \mathrm{p}<0.004$ for tilt around $\mathrm{y}-$ axis), and slightly lower for decentration, not reaching statistical significance, (slope: 0.62 , $\mathrm{r}=0.67, \mathrm{p}<0.22$, for decentration on $\mathrm{x}$-axis; slope: $-0.35, \mathrm{r}=-0.44, \mathrm{p}<0.46$ for decentration on y-axis). Figure 3.4 shows a Bland-Altman plot for tilt measurements (a) and for decentration measurements (b), i.e. the average of tilt/decentration coordinates from Purkinje and OCT versus difference of tilt/decentration coordinates from both methods. The average difference in tilt from both methods was $-0.17 \mathrm{deg}$, with the $95 \%$ limits of agreement going from -0.754 to $0.416 \mathrm{deg}$. All tilt coordinates from either method were within the confidence interval, and $40 \%$ differed by less than $0.5 \mathrm{deg}$. The average difference in decentration from both methods was $-0.086 \mathrm{~mm}$, with the $95 \%$ limits of agreement going from -0.205 to $0.033 \mathrm{~mm}$. All decentration coordinates from either method were within the confidence interval, and $50 \%$ differed by less than $0.1 \mathrm{~mm}$. 


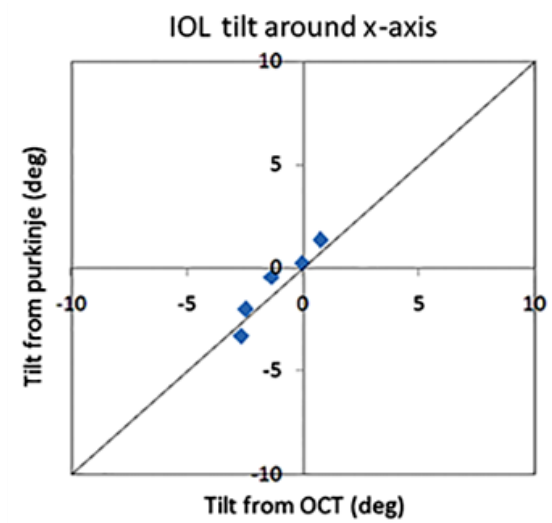

(a)

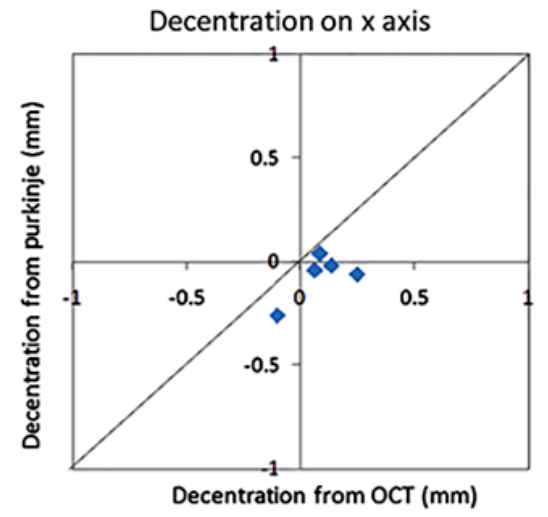

(c)

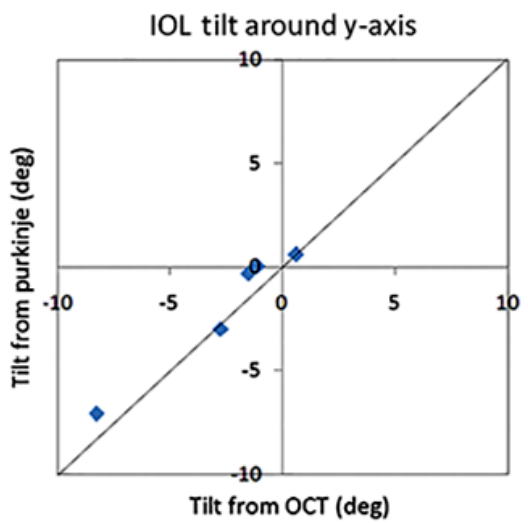

(b)

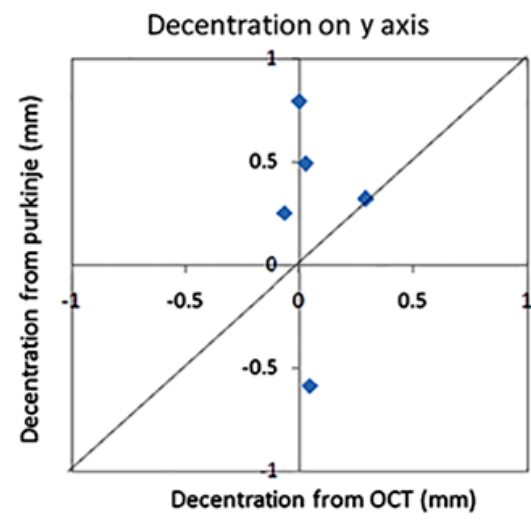

(d)

Figure 3.3 Correlation between data obtained from Purkinje-like and OCT methods for IOL tilt $(a, b)$ and decentration (c, d) in all 5 eyes of the study.

While all estimates are obtained from the same image data set, the causes for the slight discrepancies may arise from the 3D nature of the reference axes in OCT and the 2D nature in Purkinje. The center of the pupil in the OCT images is given by lateral and axial coordinates, which may result in slightly different pupillary axes. Besides, the pupil plane itself may show some tilt, while the pupil edges in the Purkinje-like method are obtained from the frontal projection (2D coordinates) in the en-face OCT images. We simulated the Purkinje images of a computer 3D model eye built using the estimates for tilt, decentration, and eye rotation obtained from the Purkinje-like method using ZEMAX and found that the positions of the simulated PI, PIII and PIV were within $0.004 \mathrm{~mm}, 0.011 \mathrm{~mm}$ and 0.004 $\mathrm{mm}$, respectively, from the experimental Purkinje images (see Figure 3.5 showing an example from eye S\# 8 OS). 


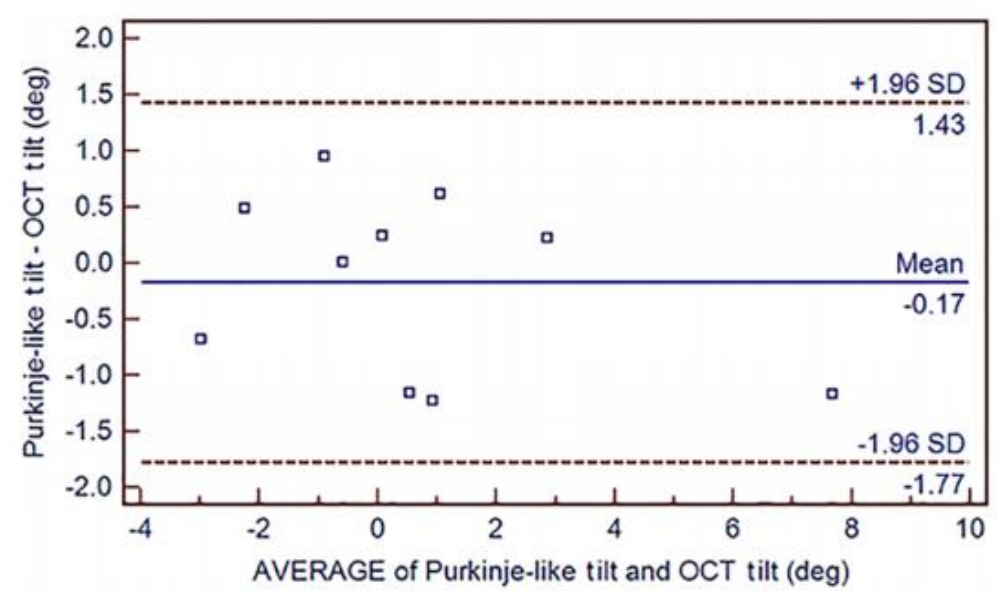

(a)

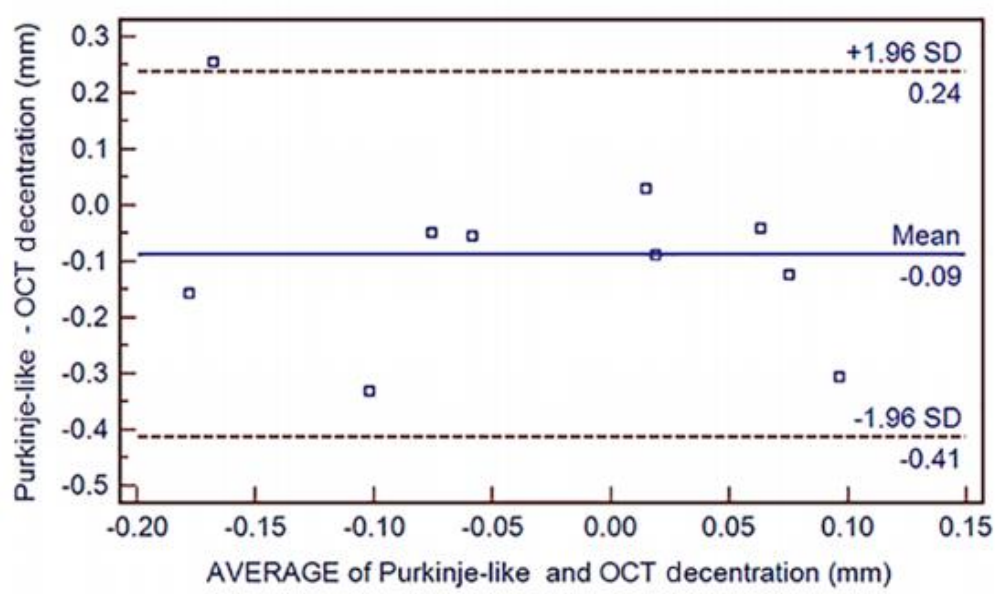

(b)

Figure 3.4 Bland-Altman plot-differences between Purkinje-like and OCT estimates of tilt (a) and decentration (b). Horizontal lines represent the mean difference $(-0.17 \mathrm{deg}$ and $-0.09 \mathrm{~mm}$, respectively) and $95 \%$ limits of agreement.

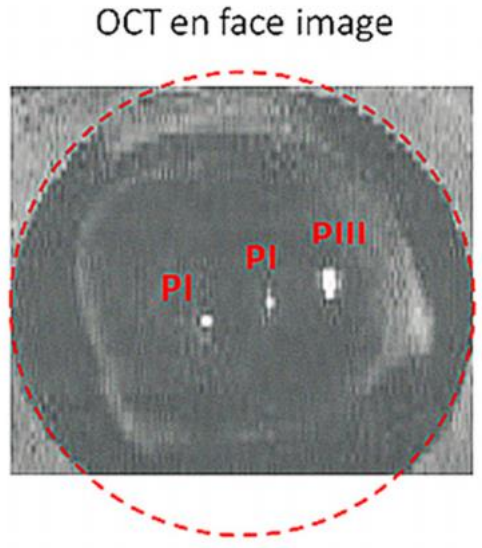

(a)
Purkinje images simulation

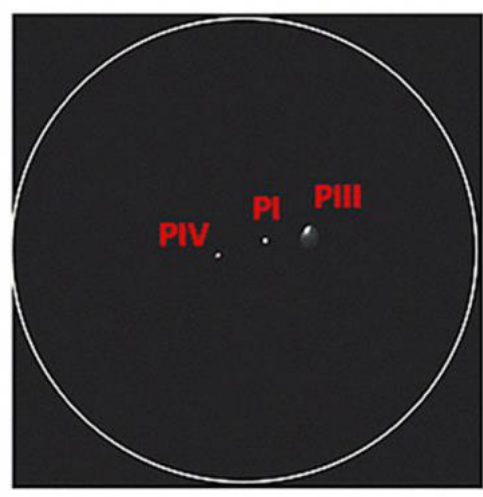

(b)

Figure 3.5 Experimental en-face OCT image (a) and simulated (b) PI, PIII and PIV, for S\# 8 OS 


\subsection{Discussion}

We have developed and validated a new method of measurement of IOL tilt and IOL decentration from en-face OCT anterior segment images showing Purkinje-like reflections from the anterior cornea and anterior and posterior lens surfaces. The images are similar to those obtained by standard Purkinje method images, which are specular reflections formed at the ocular surfaces, and matched in position. The Purkinje-like reflex locations are assumed to be linear combinations of eye rotation, lens tilt and decentration, with the weighting coefficients dependent on the eye's biometry (and easily derived by computations on computer eye models), lens tilt and decentration. The method has been tested in an artificial model with and without water, and showed accuracies similar to those of standard Purkinje imaging methods.

The proposed methodology shares analysis with the standard Purkinje based method, where pupil conjugate images are retrieved by the use of a CCD camera provided with a telecentric lens. However, the availability of tomographic data from OCT allows for unequivocal identification of the corresponding ocular surface producing the reflection, with no limitations arising from potential overlapping of the Purkinje images (one of the main drawbacks of the standard method). The dense lateral sampling of our spectral OCT configuration prevents limitations produced by sparse meridional sampling (such as that used in Scheimpflug instruments). Besides, the availability of geometrical and biometrical information (corneal keratometry, lens phakometry, anterior chamber depth), required in the Purkinje method and normally gathered from other instruments in the standard Purkinje approach, can be retrieved from the same 3D OCT data set. Despite the high acquisition rates of the OCT system, the collection of 3D en-face OCT images in our current configuration is significantly slower $(0.72 \mathrm{~s})$ than the Purkinje method with a standard CCD camera $(30 \mathrm{~Hz})$. This imposes limitations to the dynamic measurement of IOL tilt and decentration, which may be of interest in some applications (Juan Tabernero 2012). High acquisition rates are important to avoid potential errors imposed by eye motions. However, it is conceivable to limit the acquisition of OCT cross-sections in selected regions, which would considerably diminish acquisition times.

We have compared the tilts and decentrations measured directly from fan/optical distortion corrected OCT images with those obtained from the en-face OCT Purkinje-like method, and found, in general, high agreement between the data, particular for tilt. We have identified slight differences in the definition of the pupillary axis as a potential source of the discrepancies. However, using the appropriate references in a computer eye model should result in the description of the same eye.

Measurements of tilt and decentration of IOLs, along with biometry and anatomical parameters, are of great value for evaluating optical quality in patients after cataract surgery, and also for understanding their relative role in optical degradation. Furthermore, measurements of IOL tilt and decentration in patients will help to optimize IOL and haptic design. Tilt and decentration are therefore critical parameters when building customized computer eye models that can predict optical performance of IOLs (Tabernero, Piers et al. 2006, Rosales and Marcos 2007). The quantitative 3D anatomical information provided by OCT allows building these eye models with all geometrical and biometric 
information obtained from a single instrument. Anterior 3D OCT images allow direct estimation of IOL tilt and decentration, but the analysis requires correction of both fan and optical distortion (Ortiz, Siedlecki et al. 2010). However, the Purkinje method only requires knowledge of axial biometry, anterior corneal radius of curvature, and IOL radii of curvature, easily accessible following fan distortion correction of the images and from geometrical data of the IOL.

The amounts of tilt and decentration found in this chapter $(3.30 \pm 4.68 \mathrm{deg}$ and $0.16 \pm 0.16$ $\mathrm{mm}$, respectively) are comparable with those found in prior literature $(3.01 \pm 1.39 \mathrm{deg}$ and $0.38 \pm 030 \mathrm{~mm}$ by (de Castro, Rosales et al. 2007); $4.30 \pm 2.67 \mathrm{deg}$ and $0.45 \pm 0.0 .26 \mathrm{~mm}$ by (Wang, Woung et al. 1998); 3.73 $\pm 1.18 \mathrm{deg}$ and $0.45 \pm 0.23 \mathrm{~mm}$ by (Kim and Shyn 2001)) in eyes implanted with standard monofocal IOLs. However, the lack of clear symmetry between right and left eyes and, particularly, the large tilts found in some eyes (i.e. S\#3 OD) are likely associated to the fact that A-IOLs with hinged haptics may experience larger misalignments than standard monofocal IOLs (Marcos, Ortiz et al. 2013), among other IOLs due to asymmetric fibrosis in the haptic region (Z-syndrome in Crystalens) (Yuen, Trattler et al. 2008).

In summary, robust measurements of IOL tilt and decentration can be obtained from a Purkinje-like analysis on a volumetric set of anterior segment OCT data. OCT-based corneal topography data, IOL alignment and eye rotation data from the Purkinje method and IOL geometry can be used in customized pseudophakic eye models allowing estimates of the optical quality and the factors contributing to optical degradation in these eyes. The Purkinje-like method allows characterization of the eye rotation, and lens tilt and decentration, giving insights on the performance of implanted A-IOLs. These data, along with biometric data obtained from OCT, can be easily transferred into computer eye models for evaluation of the contributed factors to optical degradation in eyes implanted with IOLs and the impact of IOL misalignment in particular.

In this chapter, we have described an OCT-based method to measure tilt and decentration of the crystalline lens and IOLs implanted in cataract subject. On the other hand, OCT can be used to assess the shape of the natural lens. In following chapters these technologies will be applied to study lens topography and the impact of IOL tilt on the optical quality in eyes implanted with IOLs. 


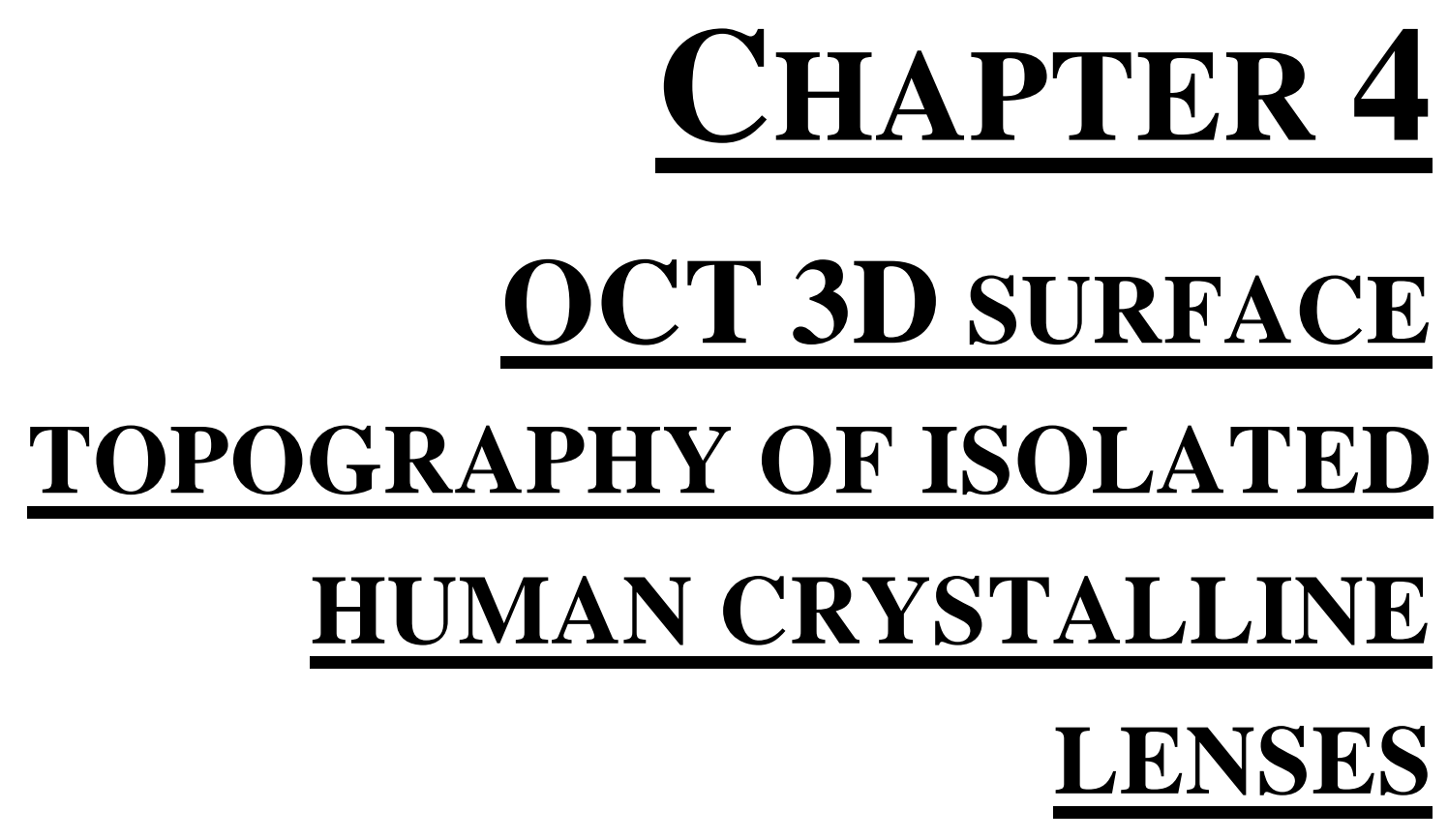


This chapter is based on the following publication:

M. Sun, J. Birkenfeld, A. de Castro, S. Ortiz and S. Marcos (2014). "OCT 3D surface topography of isolated human crystalline lenses." Biomed Opt Express 5(10): 3547-3561.

The contribution of Mengchan Sun to the study, in collaboration with other coauthors, was the literature search, the design of the experiments and the data acquisition (in collaboration with Alberto de Castro), the development of specific routines and the analysis and processing of the data. This work was also presented at the 2014 Annual Meeting of the Association for Research in Vision and Ophthalmology (ARVO) in Orlando (Florida, USA) 


\section{RESUMEN}

Objetivos: En este capítulo presentamos la medida de la topografía de superficies del cristalino del ojo humanas aislado y una evaluación de la relación entre las formas de superficie anterior y posterior del cristalino y sus cambios con la edad.

Métodos: La Tomografía de Coherencia Óptica Cuantitativa 3-D se utilizó para medir la topografía superficial de 36 lentes humanas aisladas y para evaluar la relación entre la forma de la superficie anterior y posterior del cristalino y sus cambios con la edad. Los cristalinos se co, colocaron en una cubeta en un medio de conservación (DMEM / F-12, HEPES, GIBCO, transparente). Las lentes se midieron con un sistema OCT de desarrollo propio. Una imagen completa en 3D contiene 1668 A-scans y 60 B-scans en una región de $12 \times 12 \mathrm{~mm}$. La resolución axial del OCT es de $6.9 \mu \mathrm{m}$ en el tejido. Todas las superficies de la lente se ajustaron a los polinomios Zernike de orden 6. Los cambios en las superficies del cristalino con la edad y la relación entre las superficies de las anterior y posterior se estudiaron mediante regresiones lineales entre los parámetros que describen la forma de la superficie (radios de curvatura, constantes cónicas, coeficientes polinomiales de Zernike y RMS de alto orden) y la edad. Los niveles de significación estadística se ajustaron mediante una corrección de Bonferroni, aplicada para evitar posibles errores de tipo 1 derivados de comparaciones múltiples.

Resultados: El astigmatismo es la aberración superficial predominante en las superficies anterior y posterior del cristalino (representando aproximadamente el 55\% y el 63\% de la varianza, respectivamente), seguido de términos esféricos, coma, "trefoil" y "tetrafoil". La magnitud de astigmatismo de la superficie anterior y posterior no varía significativamente con la edad. El ángulo relativo entre los ejes de astigmatismo superficial anterior y posterior fue de 36.5 grados en promedio, con tendencia a disminuir con la edad, siendo mayor de 45 grados en $36.1 \%$ de los cristalinos. La RMS de aberración esférica, coma y aberraciones de tercer orden de los mapas de elevavción anterior y posterior) disminuye significativamente con la edad. También encontramos una correlación estadísticamente significativa entre los términos de orden 3 y 4 de las superficies anterior y posterior del cristalino.

Conclusiones: La comprensión de la coordinación de las geometrías de la superficies anterior y posterior del cristalino y sus cambios topográficos con la edad arrojan luz sobre el papel de la lente en las propiedades ópticas del ojo y el mecanismo de envejecimiento del cristalino. 


\subsection{Introduction}

The crystalline lens of the eye is a fascinating optical element which, together with the cornea, transmits and refracts light to form an image of the world on the retina. The elastic young crystalline lens changes shape via constriction and/or relaxation of the ciliary muscle, resulting in a change of the optical power of the eye which provides a focused image of objects both near and far. With aging, the human lens becomes thicker, relatively steeper and loses the ability to accommodate as we maintained in Section 1.2.3. In addition, the crystalline lens shows a gradient refractive index (GRIN) distribution, which also changes with age. Recent work in the VioBio lab has shown that in human (Birkenfeld, de Castro et al. 2014), as well as monkey (de Castro, Birkenfeld et al. 2013) and porcine lenses (Birkenfeld, de Castro et al. 2013), both the lens shape and the GRIN play a role in the negative spherical aberration of the lens. Despite its important contribution to the optical quality of the eye, several aspects of the crystalline lens are not known. The lens surface radii of curvature and their changes with age and accommodation have been extensively measured both ex vivo and in vivo. Data ex vivo and in vivo differ primarily because the isolated lens appears in its maximally accommodated state, and therefore the young lens shows large steepening ex vivo. Ex vivo, the anterior and posterior lens surface radii of curvature tend to increase with age while both surfaces tend to steepen with age in vivo. (More detail about previous research can be found in Section 1.2.3)

Given its high accessibility, the corneal topography is well characterized. Corneal irregularities in the elevation map and astigmatism are common. Interestingly, the posterior corneal surface appears to compensate for part of the errors of the anterior cornea. In particular, the posterior cornea has been reported to compensate around 3.5\% of the coma of the anterior surface (Dubbelman, Sicam et al. 2007). There are also numerous reports of the compensation of the corneal astigmatism by the astigmatism of the crystalline lens using corneal and refractive parameters as we maintained in Section 1.2.2. However, to our knowledge, there is little information on possible compensation within the crystalline lens itself, i.e. between the anterior and posterior surfaces or between its surfaces and its GRIN. In this chapter, we have measured the elevation maps of the anterior and posterior lens surfaces of 36 isolated human donor lenses of different ages with quantitative 3D OCT. These ex vivo measurements allow direct access to the posterior surface of the lens, without potential distortions produced by the GRIN of the lens. To our knowledge, this is the first systematic study of the lens topography (including high order irregularities) and its change with age. Knowledge of the full lens topography and its age-related changes is important in the understanding of the relative contribution of the lens properties to the optical quality of the eye, possible through the use of ray tracing on custom eye models, as well as in investigating the changes undergone by the lens with aging. This information is particularly relevant for the formation of correcting strategies in the eye, and specifically in cataract surgery, in which an artificial intraocular lens replaces the crystalline lens of the eye. Novel designs aim at finding inspiration from the natural young lens. 


\subsection{Methods}

\subsubsection{Lens specimens and preparation}

We introduced lens specimen preparation in Section 2.5.1. As a brief summary, 36 isolated human crystalline lenses from 28 human donors were obtained from the BST Eye bank (Banc de Sang i Teixits, Barcelona, Spain). The post-mortem time varied between 1 and 3 days at the time of the experiment. A typical experimental session lasted 1-2 hours.

All lenses were imaged in a cuvette and immersed in preservation medium. 3D OCT imaging was performed with the anterior lens surface facing the OCT beam (anterior up image), and care was taken that the lens did not appear tilted in the real-time OCT image. The lens was then flipped around a predetermined horizontal axis (the same for all lenses) to image it again, this time with the posterior surface facing the OCT beam (posterior up image). The procedure is similar to that described in prior publications (Borja, Siedlecki et al. 2010, de Castro, Ortiz et al. 2010). The lenses were imaged in 3D using the custom developed OCT system described in Section 2.1.2. One set of OCT images contains 1668 A-Scans and $70 \mathrm{~B}-\mathrm{Scans}$ on a $12 \times 12 \mathrm{~mm}$ lateral range. The acquisition time for each OCT image was $4.5 \mathrm{~s}$. Each set of 3D images consisted of two acquisitions at two different planes of focus which were merged to produce a complete image of the anterior surface, posterior surface and cuvette holding the lens, using customized Matlab programs (More detail described in Section 2.6.2).

\subsubsection{Experimental sOCT setup and experimental protocols}

The crystalline lenses were placed in a cuvette filled with a preservation medium (DMEM/F-12, HEPES, no phenol red, GIBCO) (Augusteyn, Rosen et al. 2006). The lenses were measured with a custom developed OCT system, described in more detail in Section 2.1.1. One complete 3D image was composed by $1668 \mathrm{~A}$-scans and $60 \mathrm{~B}$-scans on a $12 \times 12$ $\mathrm{mm}$ region. The axial resolution was calculated to be $6.9 \mu \mathrm{m}$ in tissue.

The lens was immersed in the cuvette and placed on a ring. Centration of the lens for image acquisition was done using the visualization mode provided by the OCT, which displayed on the computer screen every 0.5 seconds two perpendicular B-scans passing through the OCT axis. The lens axis was aligned with the OCT scan axis so that a specular reflection was seen from both surfaces of the lens. The lens was first imaged with the anterior surface facing the OCT beam, and was then flipped around a predetermined axis and imaged again with the posterior surface up. To produce a complete image of the crystalline lens, two images were captured in each position, one with the OCT focused on the first visible surface (anterior in the anterior-up image and posterior in the posterior-up image), and another with the OCT focused on the second surface, with image showing the cuvette holding the lens and the preservation media. The procedure was described in more detail elsewhere (Birkenfeld, de Castro et al. 2014) 


\subsubsection{Lens surface elevations}

Only the first lens surface imaged in each condition was analyzed in terms of elevation: anterior surface in anterior-up images and posterior in posterior-up images. The distortions present in this surface arise from the scanning architecture of the OCT system (fan distortion (Ortiz, Siedlecki et al. 2010) in Section 2.1.2) and the refractive index of the preservation medium, were corrected. The other two surfaces visible in the OCT image were the second surface of the lens (posterior in anterior-up images and anterior in posterior-up images) and the cuvette. These two surfaces are also distorted by the unknown optics of the crystalline lens. The second surface of the lens was not used in this chapter but the image of the cuvette was used for alignment and to avoid possible rotations between the anterior-up and posterior-up images produced while flipping the lens (Birkenfeld, de Castro et al. 2014).

To obtain the shape of the lens surfaces, a surface segmentation MATLAB algorithm, previously described in Section 2.6.2, was used. The segmented surfaces were fit to biconical surfaces and spheres. The apex of the lens was used as a reference to center each surface. As we maintained in Section 2.6.2, lens surface elevation maps (obtained by subtraction of the best fitting spheres from the segmented surfaces) were fitted to $6^{\text {th }}$ order Zernike polynomials. All fittings were done for a 6-mm diameter optical zone. Descriptive parameters of the lens surface geometries were radii of curvature and asphericities, and descriptive parameters of the surface elevation maps included individual surface Zernike coefficients (up to $6^{\text {th }}$ order) and the RMS combination of some terms (RMS astigmatism, RMS trefoil, RMS tetrafoil, RMS spherical terms, RMS coma and RMS $4^{\text {th }}$ order Astigmatism) or Zernike orders (from $2^{\text {nd }}$ order to $6^{\text {th }}$ order RMS). We studied the changes of those parameters with age as well as relationships between the anterior and the posterior surfaces.

\subsubsection{Lens orientation}

As the relative angle of the lens with respect to the cornea and the body is unknown, for convention, all lenses were aligned such that the astigmatic axis of the anterior lens was on the vertical meridian (direct astigmatism). The magnitude of astigmatism (C) of each lens surface was computed using Eq. 4.1.

$C=\left(n_{2}-n_{1}\right) \times\left(\frac{1}{R_{x}}-\frac{1}{R_{y}}\right)$

Where $R_{x}, R_{y}$ are the radii of curvature as obtained from the biconic fittings, and $n_{1}$ and $n_{2}$ are the refractive indices of the lens' surrounding medium and the lens cortex, respectively. A lack of reports of the index of refraction of the capsular bag prevented us from using that value in the calculations. Instead, and in keeping with previous publications (Dubbelman and Van der Heijde 2001, Borja, Manns et al. 2008), we used a surface refractive index of 1.3709. The refractive index of aqueous was assumed to be equal to the refractive index of water $(1.332$ at $635 \mathrm{~nm})$. The angle $\alpha$ of astigmatism of each surface was given by 
$\alpha=\tan ^{-1}\left(\frac{J_{45}}{J_{180}}\right) / 2$

Where $J_{180}$ is the power at axis $\alpha=0^{\circ}=180^{\circ}$ and $J_{45}$ is the power at axis $\alpha=45^{\circ}$ (Thibos and Horner 2001). The image of the cuvette was used to align the anterior-up and posteriorup images (Birkenfeld, de Castro et al. 2014). All OCT images were processed to obtain the shape of the lens surfaces and the surface of the cuvette, using algorithms described in detail in previous publications (de Castro, Siedlecki et al. 2011). We aligned the anterior and posterior surfaces using the astigmatism vector of the cuvette segmented surface. This adjustment guaranteed that the lens could be treated as a whole, and therefore a rotation of the entire lens preserved the relative angle between anterior and posterior surface astigmatic axes. The entire lens was rotated so that $\alpha_{\text {anterior }}=90 \mathrm{deg}$ for the anterior lens, while keeping the astigmatism relative angle ( $\left.\alpha_{\text {anterior _ }} \alpha_{\text {posterior }}\right)$ constant.

Elevation maps and individual Zernike terms are provided following rotation according to this convention (i.e. $Z_{2}^{-2}=0$ in all anterior lenses). Posterior elevation maps are shown by flipping the horizontal coordinates (i.e. by moving the observation point to the back of the lens). Since the used convention still leaves the vertical orientation as an unknown (i.e. the lenses could be rotated $180 \mathrm{deg}$ ) and we cannot distinguish between right and left eyes, the relative angles of astigmatism between the anterior and posterior surfaces are given in a 0 90 deg range.

\subsubsection{Power vector analysis}

To illustrate the distribution of anterior and posterior astigmatism in the lens sample, we present the magnitude of astigmatism and the relative angle between anterior and posterior astigmatism in a power vector graph (Thibos and Horner 2001, Liu, Chou et al. 2011). The length of the vectors represents the calculated magnitude of surface astigmatism (in diopters), and the directions of the vectors correspond to the relative angle between the anterior and posterior astigmatism axes. All vectors are represented in a polar coordinate system. We studied the age-dependence of the magnitude of astigmatism of the anterior and posterior surfaces as well as of the relative angle of astigmatism between those surfaces.

\subsubsection{Correlation analysis}

Changes in lens surfaces with age and the relationship between the anterior and posterior lens surfaces were studied by linear regressions between the parameters describing the lens surface shape (radii of curvature, conic constants, Zernike polynomial coefficients and high order RMS) and age, and also between the descriptive parameters of the anterior and posterior lens surfaces. The statistical significance levels were adjusted by a Bonferroni correction, applied to avoid potential Type 1 errors arising from multiple comparisons. The significance level was established at $\mathrm{p}<0.05$ for radii of curvature and conic constants, $\mathrm{p}<0.008$ for RMS terms, $\mathrm{p}<0.01$ for RMS orders and $\mathrm{p}<0.00017$ for individual Zernike terms. 


\subsection{Results}

\subsubsection{Lens surface elevation maps}

Figure 4.1 shows the two central raw OCT images that need to be merged to obtain a complete anterior-up image of one of the crystalline lens, segmented 3D lens and cuvette surfaces. The fan distortion and the optical distortion due to the DMEM are corrected, and the shape of the cuvette is used to avoid rotations between anterior-up and posterior-up imaging.

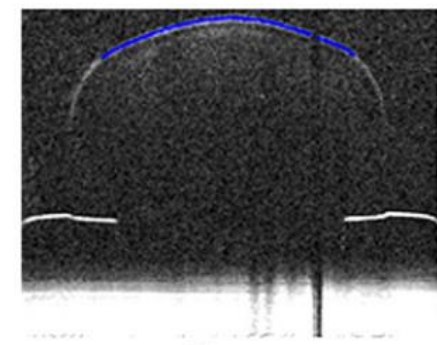

(a)

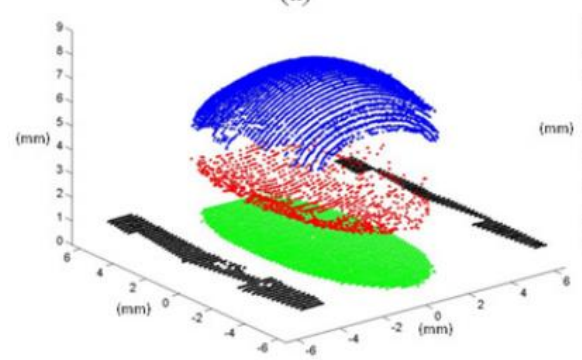

(c)

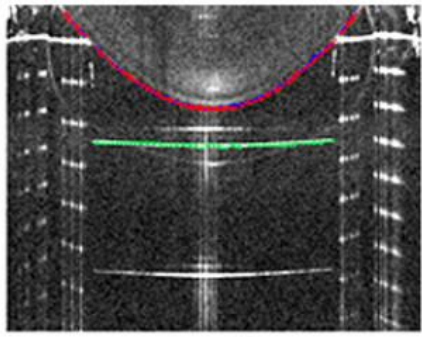

(b)

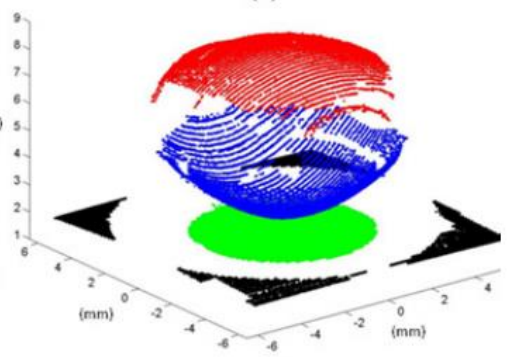

(d)

Figure 4.1 Upper panels: OCT images corresponding to the anterior-up position of one of the crystalline lenses imaged, with (a) OCT focused on the anterior surface and (b) OCT focused on the posterior surface and the image of the cuvette. The detected surfaces are marked in blue (anterior surface), red (posterior surface) and green (cuvette). Lower panels: 3D OCT data from images of the crystalline lens with (c) the anterior surface up and (d) the posterior surface up. The blue and red points correspond to the segmented anterior and posterior surfaces of the lens, respectively. The green points and the black points correspond to the cuvette imaged through the lens, therefore distorted by the lens, and the cuvette without distortion respectively.

Figure 4.2 shows Zernike fittings of the anterior and posterior lens surface elevations (relative to the best fitting sphere) for all lenses of the chapter, sorted by age. The first and third columns show the astigmatic Zernike terms only, and the second and fourth columns represent astigmatism and HOA (up to $6^{\text {th }}$ order terms) of the anterior and posterior surfaces respectively. Since, by convention, the lenses have been aligned such that the anterior lens' steepest meridian is vertical, the anterior maps show only the $Z_{2}^{2}$ astigmatic component. In many cases the astigmatic axis of the posterior lens surface is rotated with respect to the anterior lens surface. Astigmatism dominates in some, but not all eyes. In general, there is a good correspondence between anterior and posterior lens surface maps. Also, a certain degree of similarity occurs in the HOA maps of both eyes from the same donor, marked with asterisks in the figure (lens\#7\&8, lens\#11\&12, lens\#15\&16, lens\#17\&18, lens\#23\&24, lens \#27\&28, lens \#35\&36). For eye pairs from the same donor, the average correlation coefficients for the high order Zernike between eyes are $r=0.78(p=0.002)$ for 
the anterior and $\mathrm{r}=0.53(\mathrm{p}=0.03)$ for the posterior lens surface, while the average correlation coefficients when compared to eyes from other eyes are $r=0.10$ and $r=0.17$ for the anterior and posterior lens surfaces, respectively.
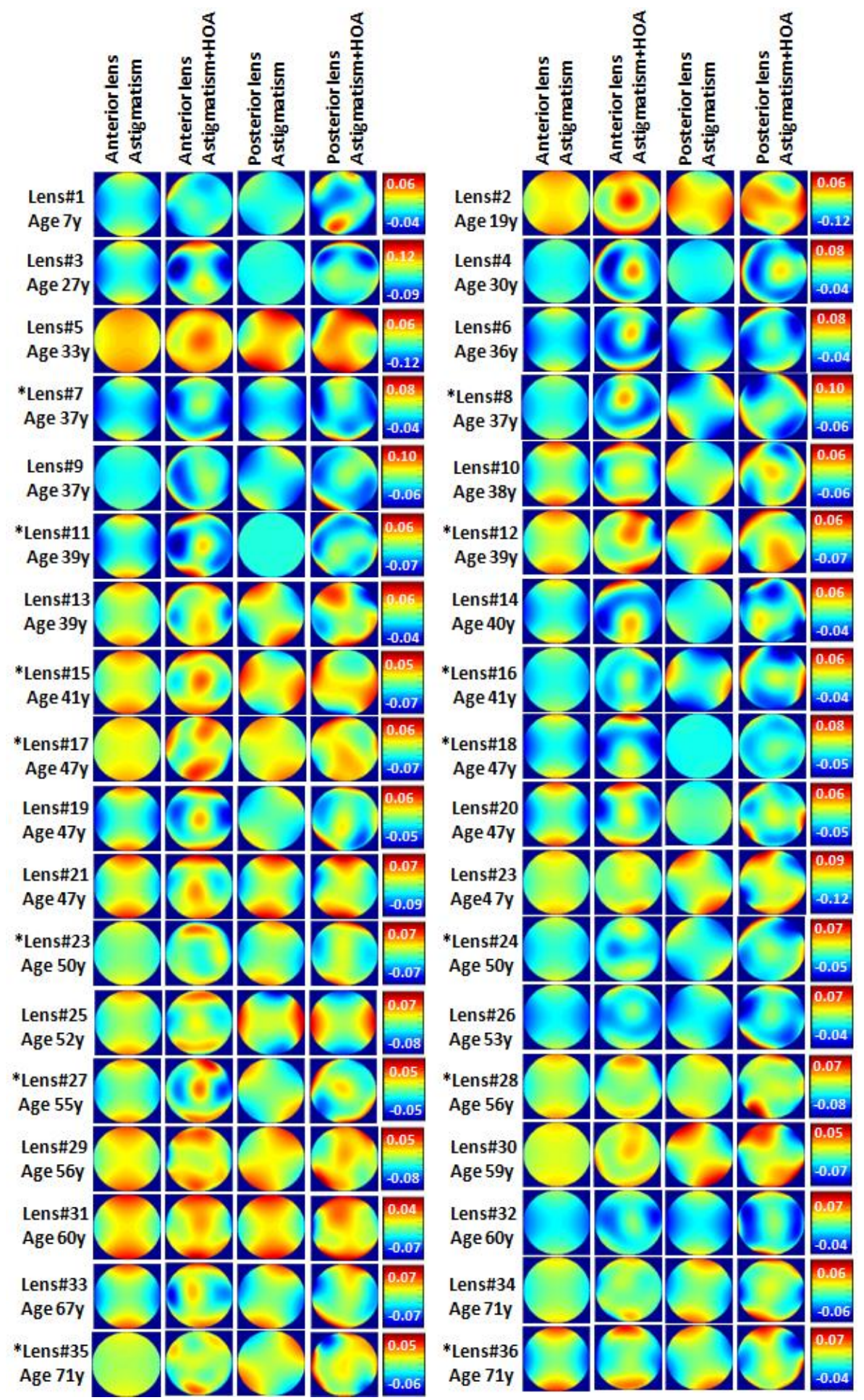

Figure 4.2 Lens surface elevation maps for all lenses, ordered by age. By convention, the maps are aligned so that the steepest meridian of the anterior lens surface lies on the vertical axis. Anterior and posterior images are shown as mirrored on the vertical axis. Asterisks indicate pairs of lenses from the same donor. 


\subsubsection{Lens radii of curvature and asphericity: anterior/posterior lens relationships and changes with age}

Figure 4.3 shows the radii of curvature of the steepest and flattest meridians ( $R_{s}$ and $R_{f}$ ) of the anterior and posterior lens surfaces as a function age, obtained from biconic fittings. Per definition, the steepest meridian is aligned vertically on the anterior lens surface, and the steepest and flattest meridians are orthogonal on both surfaces.

Similar to earlier reports of the average radii of curvature, both the radii of curvature in the flattest and steepest meridians increase significantly with age $(\mathrm{p} \leq 0.003)$. The steepest and flattest meridians change at a rate of $95 \mu \mathrm{m} /$ year and $94 \mu \mathrm{m} /$ year, respectively, in the anterior lens, and $20 \mu \mathrm{m} /$ year and $19 \mu \mathrm{m} /$ year in the posterior lens.

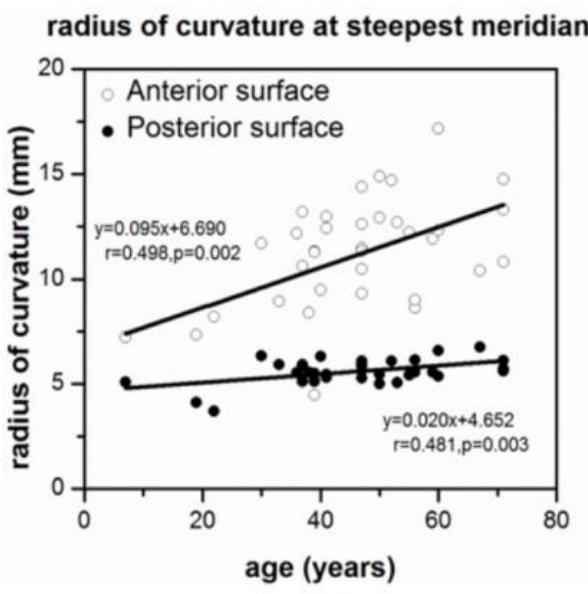

(a)

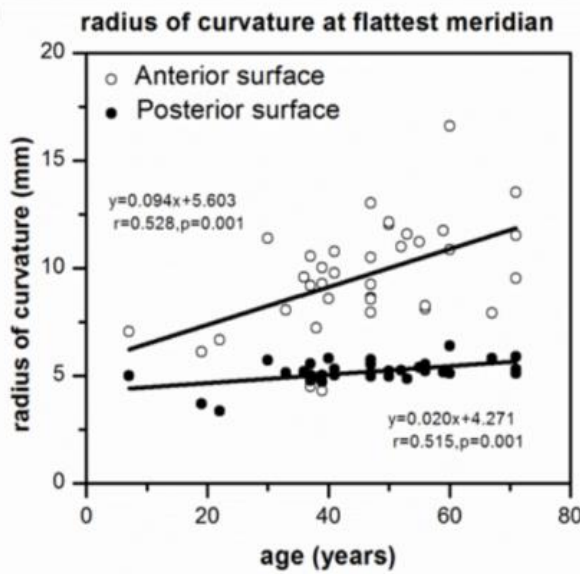

(b)

Figure 4.3 (a) Radii of curvature of anterior and posterior lens surfaces at their corresponding steepest meridian as a function of age (b) Radii of curvature of anterior and posterior lens surfaces at their corresponding flattest meridian as a function of age. Data was obtained from fits to biconic surfaces. Open circles represent data of the anterior lens surface and solid circles represent data of the posterior lens surface.

We also studied the change in conic constant $(\mathrm{k})$ with age. Figure 4.4 shows the conic constants in the steepest and flattest meridians for the anterior and posterior lens surfaces obtained from biconic fittings. Both anterior and posterior lens conic constants shift from negative values towards more positive (or less negative) values with age, although the change is not statistically significant. 


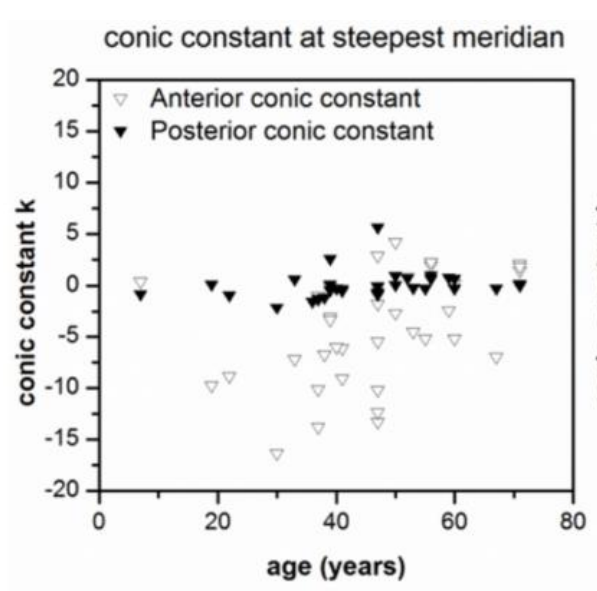

(a) conic constant at flattest meridian

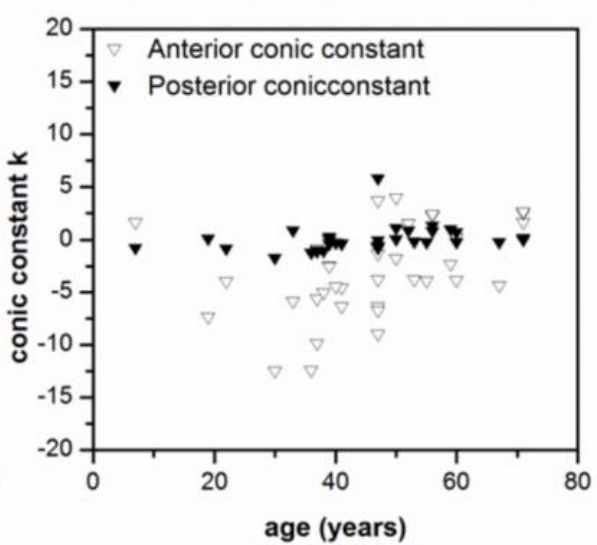

(b)

Figure 4.4 (a) Conic constants of anterior and posterior lens surfaces at their corresponding steepest meridian as a function of age. (b) Conic constants of the posterior lens surfaces at their corresponding flattest meridian as a function of age. Data was obtained from fits to biconic surfaces. Open triangles represent data from the anterior lens surface and solid triangles represent data from the posterior lens surface.

\subsubsection{Changes of lens surface astigmatism and relative astigmatic angle with age}

The magnitude of lens surface astigmatism (calculated from $R_{s}$ and $R_{f}$ in Eq. 4.1) ranged from 0.046 to $1.185 \mathrm{D}$ (average: $0.563 \pm 0.347 \mathrm{D}$ ) in the anterior lens and from 0.013 to $1.118 \mathrm{D}$ (average: $0.553 \pm 0.320 \mathrm{D}$ ) in the posterior lens. Figure 4.5 (a) shows the variation of anterior and posterior lens surface astigmatism with age. For both lens surfaces, the changes with age were not statistically significant ( $p=0.29$ and $p=0.23$ for anterior and posterior surfaces respectively). For $52.78 \%$ (anterior surface) and $41.67 \%$ (posterior surface) of the lenses the magnitude of astigmatism was between 0.2 and $0.6 \mathrm{D}$. Figure 4.5 (b) shows the correlation of anterior and posterior lens surface astigmatism, which did not reach statistical significance $(\mathrm{p}=0.226)$.

Figure 4.6 shows the power vector analysis for anterior and posterior lens surface astigmatism. By convention, the anterior lens astigmatism is aligned with the vertical steepest meridian, as shown in Figure 4.6(a). This convention is adopted as a consequence of the lack of information of the actual orientation of the lens in vivo (up, down, nasal, temporal), resulting in a relative angle of astigmatism in a range between 0 and $90 \mathrm{deg}$ (see Figure 4.6(b)).

The relative angle between anterior and posterior astigmatism was on average 36.46 deg. In $36.1 \%$ of the lenses the relative angle was $>45$ deg. Figure 4.7 shows the change of the relative angle of the astigmatic axis between the anterior and posterior lens surfaces with age. The relative angle tends to decrease with age, although the correlation is low $(r=0.305$, $\mathrm{p}=0.071)$. We did not find that the relative angle was correlated with the amount of astigmatism of either surface (not shown). 


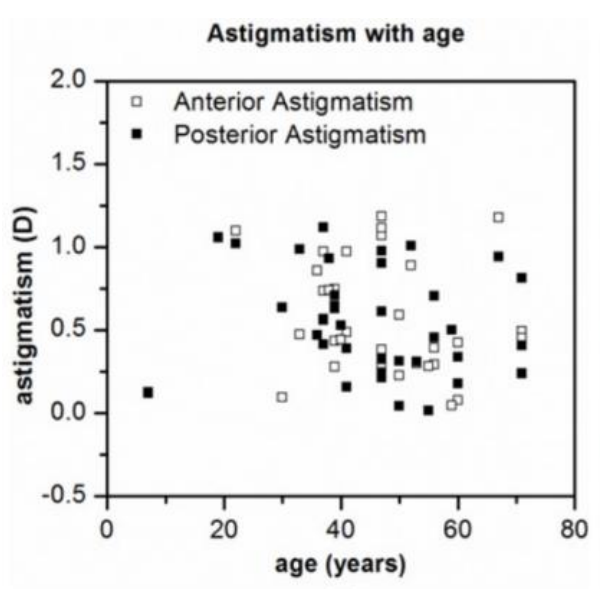

(a)

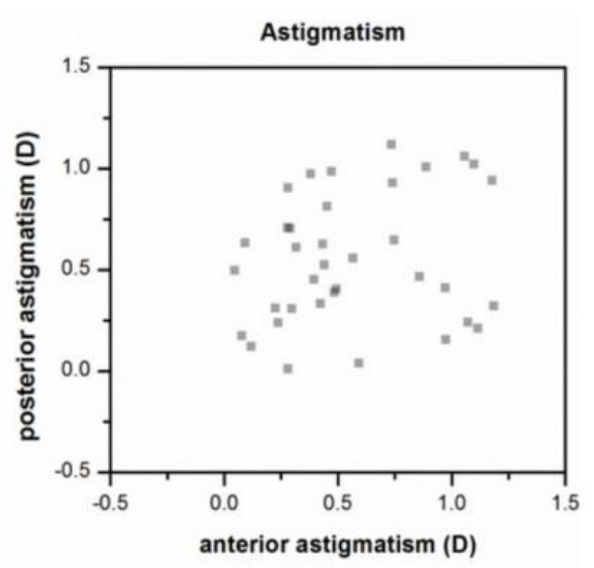

(b)

Figure 4.5 (a) Magnitude of lens surface astigmatism of the anterior and posterior lens surfaces as a function of age. (b) Correlation of the magnitude of astigmatism between anterior and posterior surfaces

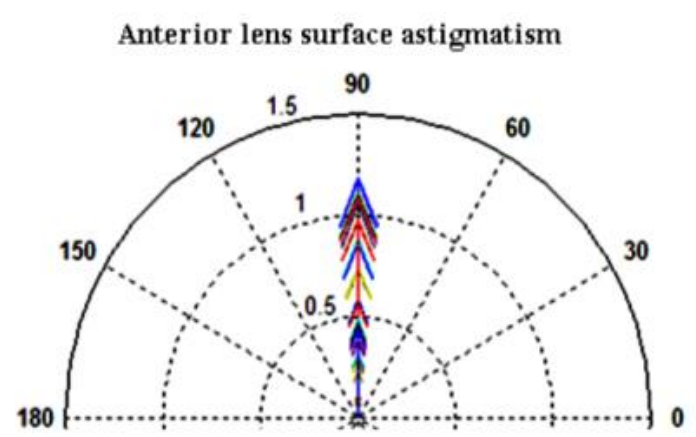

(a)

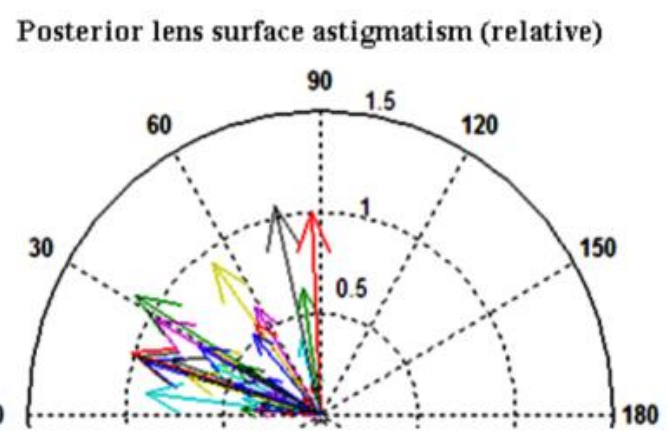

(b)

Figure 4.6 (a) Anterior astigmatism power vector polar plot with the steepest meridian aligned at $90 \mathrm{deg}$, by convention; (b) Posterior astigmatism power vector polar plot. The angle (from the vertical axis) represents the relative angle between the anterior and posterior astigmatic axis. Each arrow represents one lens; the length of the vectors represents the magnitude of the corresponding astigmatism in diopters. 


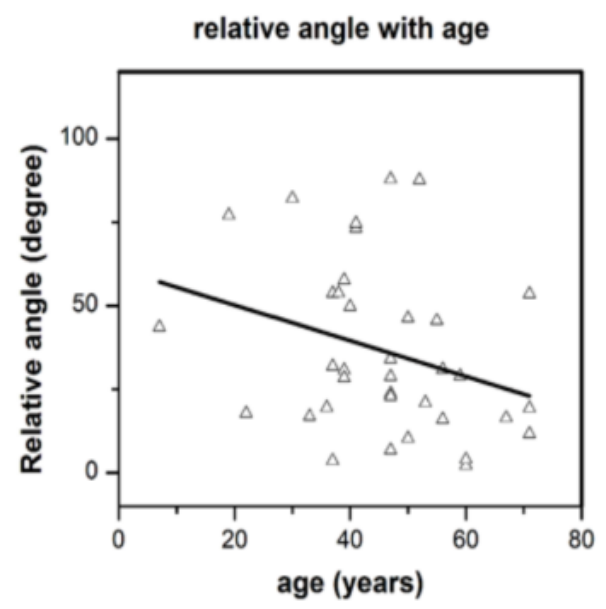

Figure 4.7 Relative angle between anterior and posterior lens surface astigmatic axis as a function of age (Slope: $-0.53 \mathrm{deg} /$ year; $r=0.305 ; \mathrm{p}=0.071$ ); 


\subsubsection{Lens elevation high order Zernike terms: anterior and posterior lens relationships}

We tested the similarity of anterior and posterior lens topographies by evaluating the correlation of the high order Zernike terms in the anterior and posterior lens surfaces. We found that several high order Zernike terms were correlated in a statistically significant manner in anterior and posterior lens surfaces. As an example, Figure 4.8 shows linear regressions between anterior and posterior surfaces for vertical trefoil $\left(Z_{3}^{-3}\right), 3^{\text {rd }}$ order RMS, RMS Coma and $4^{\text {th }}$ order RMS.

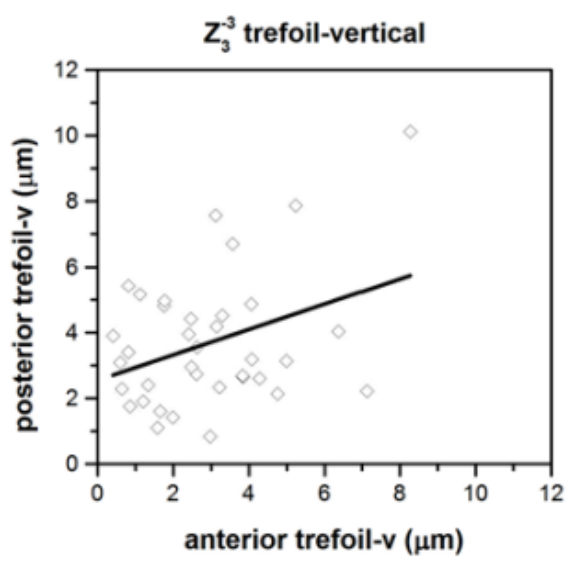

(a)

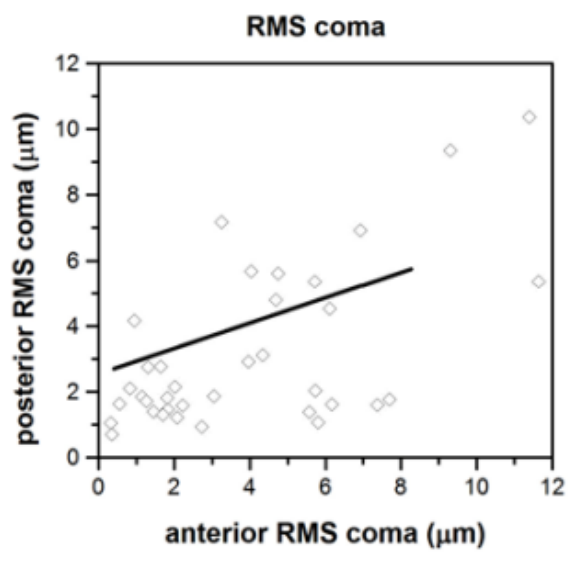

(c)

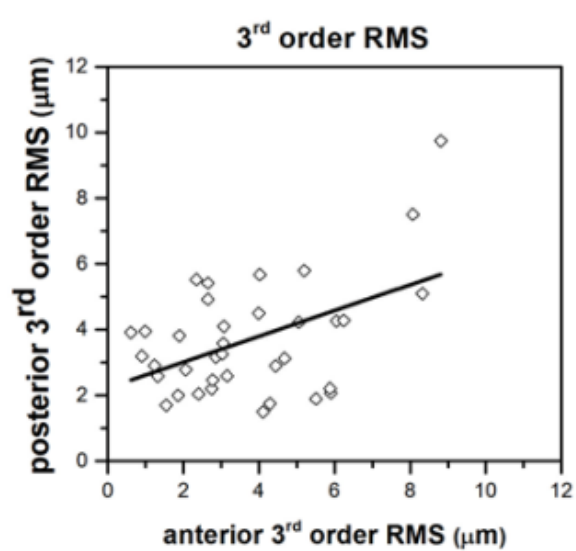

(b)

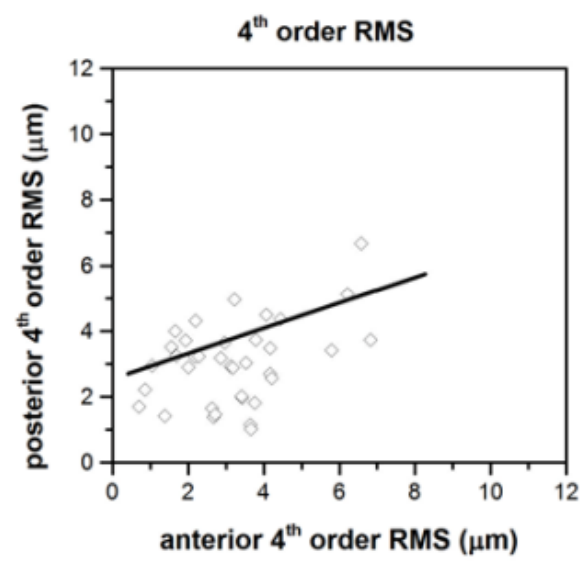

(d)

Figure 4.8 (a) Trefoil-v $Z_{3}^{-3}$ : Anterior vs. Posterior (Slope $=0.387, r=0.467, p=0.004$ ) (b) RMS $3^{\text {rd }}$ order terms: Anterior vs. Posterior (Slope $=0.019 ; \mathrm{r}=0.477, \mathrm{p}=0.003$; (c) RMS Coma: Anterior vs. Posterior $($ Slope $=0.387 ; \mathrm{r}=0.617, \mathrm{p}=0.0001)(\mathrm{d}) 4^{\text {th }}$ order RMS: Anterior vs. Posterior (Slope $=0.387 ; \mathrm{r}=0.423, \mathrm{p}=0.010$.) 


\subsubsection{Lens elevation high order Zernike terms: changes with age}

Figure 4.9 shows several Zernike surface elevation terms (in terms of RMS) as a function of age: (a) RMS coma (b) RMS spherical (c) $3^{\text {rd }}$ order RMS, and (d) $5^{\text {th }}$ order RMS, both for the anterior and posterior lens surfaces. RMS coma (Figure 4.9 (a)) with age, with a high degree of statistical significance, at a rate of $0.087 \mu \mathrm{m} /$ year and $0.123 \mu \mathrm{m} /$ year for anterior and posterior surface, respectively. RMS spherical (Figure 4.9 (b)) and $3^{\text {rd }}$ order RMS (Figure 4.9 (c)) decrease significantly with age for the anterior surface, but not for the posterior surface (RMS spherical: slope $=-0.175 \mu \mathrm{m} /$ year for anterior surface, slope $=$ $-0.05 \mu \mathrm{m} /$ year for posterior surface; $3^{\text {rd }}$ order RMS: slope $=-0.083 \mu \mathrm{m} /$ year for anterior surface). $5^{\text {th }}$ and $6^{\text {th }}$ order RMS do not show statistically significant changes with age. Table 4.1 ( $4^{\text {th }}$ and $5^{\text {th }}$ columns) shows the correlations with age for different RMS orders.

Table 4.1 Mean and standard deviation of the RMS of high-order Zernike coefficients for anterior and posterior lens surfaces. The Pearson correlation coefficient and the p-value are shown for the correlation with age of these parameters in each surface and between surfaces.

\begin{tabular}{|c|c|c|c|c|c|}
\hline $\begin{array}{l}\text { HO Zernike } \\
\text { coefficients }\end{array}$ & $\begin{array}{c}\text { anterior } \\
(\mu \mathrm{m})\end{array}$ & $\begin{array}{l}\text { posterior } \\
(\mu \mathrm{m})\end{array}$ & $\begin{array}{l}\text { anterior } \\
\text { vs. age } \\
r \text { and p }\end{array}$ & $\begin{array}{c}\text { posterior vs. } \\
\text { age } \\
\mathrm{r} \text { and p }\end{array}$ & $\begin{array}{l}\text { ant. vs. pos } \\
\text { r and p }\end{array}$ \\
\hline $\begin{array}{l}\text { RMS 3rd order } \\
\text { Zernike Terms }\end{array}$ & $\begin{array}{c}3.294 \pm \\
1.822\end{array}$ & $3.697 \pm 1.770$ & $\begin{array}{c}\mathrm{r}=0.564 \\
\mathrm{p}= \\
0.0001^{*}\end{array}$ & $\begin{array}{l}\mathrm{r}=0.373 \\
\mathrm{p}=0.025\end{array}$ & $\begin{array}{c}\mathrm{r}=0.477 \\
\mathrm{p}=0.003^{*}\end{array}$ \\
\hline $\begin{array}{l}\text { RMS 4th order } \\
\text { Zernike Terms }\end{array}$ & $\begin{array}{c}3.227 \pm \\
1.779\end{array}$ & $3.077 \pm 1.247$ & $\begin{array}{c}\mathrm{r}=0.721 \\
\mathrm{p}= \\
0.0001 *\end{array}$ & $\begin{array}{l}r=0.277 \\
p=0.102\end{array}$ & $\begin{array}{c}\mathrm{r}=0.423 \\
\mathrm{p}=0.010^{*}\end{array}$ \\
\hline $\begin{array}{l}\text { RMS 5th order } \\
\text { Zernike Terms }\end{array}$ & $\begin{array}{c}1.151 \pm \\
0.407\end{array}$ & $1.381 \pm 0.727$ & $\begin{array}{l}r=0.290 \\
p=0.087\end{array}$ & $\begin{array}{l}r=0.338 \\
\mathrm{p}=0.023\end{array}$ & $\begin{array}{l}r=0.047 \\
p=0.786\end{array}$ \\
\hline $\begin{array}{l}\text { RMS 6th order } \\
\text { Zernike Terms }\end{array}$ & $\begin{array}{c}1.216 \pm \\
0.453\end{array}$ & $1.076 \pm 0.500$ & $\begin{array}{l}r=0.149 \\
p=0.384\end{array}$ & $\begin{array}{l}r=0.061 \\
p=0.723\end{array}$ & $\begin{array}{l}r=0.236 \\
p=0.166\end{array}$ \\
\hline RMS Trefoil & $\begin{array}{c}2.553 \pm \\
1.300\end{array}$ & $3.683 \pm 2.038$ & $\begin{array}{l}r=0.266 \\
p=0.117\end{array}$ & $\begin{array}{l}\mathrm{r}=0.011 \\
\mathrm{p}=0.949\end{array}$ & $\begin{aligned} \mathrm{r} & =0.363 \\
\mathrm{p} & =0.030^{*}\end{aligned}$ \\
\hline RMS Tetrafoil & $\begin{array}{c}1.380 \pm \\
0.824\end{array}$ & $2.085 \pm 1.299$ & $\begin{array}{l}r=0.278 \\
p=0.101\end{array}$ & $\begin{array}{l}r=0.105 \\
p=0.544\end{array}$ & $\begin{array}{l}r=0.004 \\
p=0.980\end{array}$ \\
\hline RMS Spherical & $\begin{array}{c}5.219 \pm \\
5.708\end{array}$ & $5.266 \pm 3.232$ & $\begin{array}{c}\mathrm{r}=0.439 \\
\mathrm{p}=0.007^{*}\end{array}$ & $\begin{array}{l}\mathrm{r}=0.226 \\
\mathrm{p}=0.185\end{array}$ & $\begin{array}{c}\mathrm{r}=0.375 \\
\mathrm{p}=0.024^{*}\end{array}$ \\
\hline RMS Coma & $\begin{array}{c}3.440 \pm \\
2.918\end{array}$ & $3.147 \pm 2.467$ & $\begin{array}{c}\mathrm{r}=0.582 \\
\mathrm{p}= \\
0.0001^{*}\end{array}$ & $\begin{array}{c}\mathrm{r}=0.515 \\
\mathrm{p}=0.001 *\end{array}$ & $\begin{array}{c}\mathrm{r}=0.617 \\
\mathrm{p}=0.0001^{*}\end{array}$ \\
\hline $\begin{array}{l}\text { RMS 4th order } \\
\text { astigmatism }\end{array}$ & $\begin{array}{c}1.173 \pm \\
0.826\end{array}$ & $1.420 \pm 0.728$ & $\begin{array}{l}\mathrm{r}=0.189 \\
\mathrm{p}=0.270\end{array}$ & $\begin{array}{l}\mathrm{r}=0.022 \\
\mathrm{p}=0.897\end{array}$ & $\begin{array}{l}\mathrm{r}=0.174 \\
\mathrm{p}=0.310\end{array}$ \\
\hline
\end{tabular}

* Statistical significance (following Bonferroni correction) 


\subsubsection{Relative contribution of different Zernike terms to the lens surface elevations}

Figure 4.10 shows the average relative contribution of lower and higher order Zernike terms of both surfaces. Relative contributions are accounted for in terms of variance $\left(\mathrm{RMS}^{2}\right)$. Astigmatism is the predominant term both in the anterior surface $(54.96 \%)$ and in the posterior surface $(62.95 \%)$, followed by spherical $(25.10 \%, 17.85 \%)$, coma $(10.91 \%$, $6.38 \%)$, trefoil $(6.01 \%, 8.73 \%)$, tetrafoil $(1.76 \%, 2.80 \%)$ and $4^{\text {th }}$ order astigmatism $(1.27 \%$, $1.30 \%$ ), for the anterior and posterior surface, respectively. We also studied the change of these relative contributions with age (not shown) and found that for the anterior surface, the two predominant terms, astigmatism and spherical, change with age. The relative proportion of astigmatism increased with age at a rate of 0.702 /year $(r=0.427, p=0.009)$, while the percentage of spherical term decreased significantly with age at a rate of $-0.616 /$ year $(r=0.474, p=0.004)$. For the posterior surface, we only found a significant change in the proportion of coma (slope $=-0.307 /$ year, $r=0.450, p=0.006$ ). 


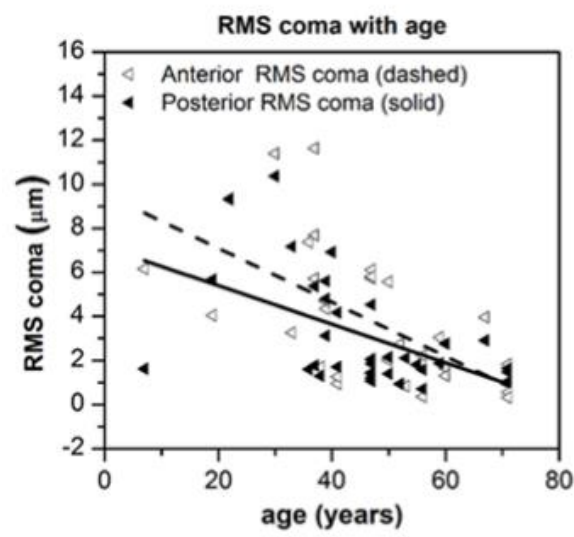

(a)

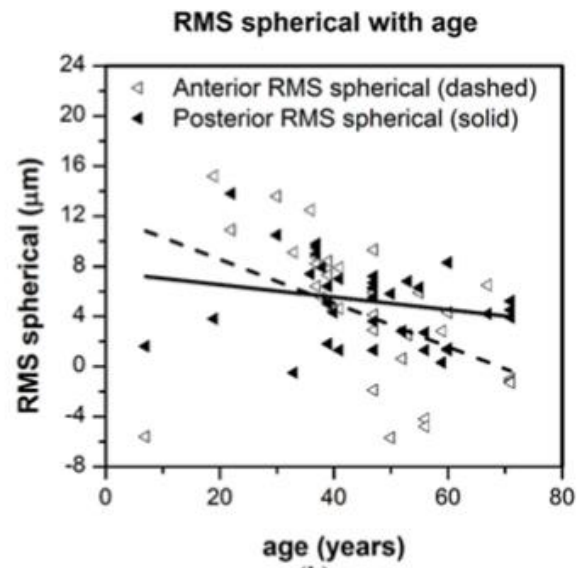

(b)

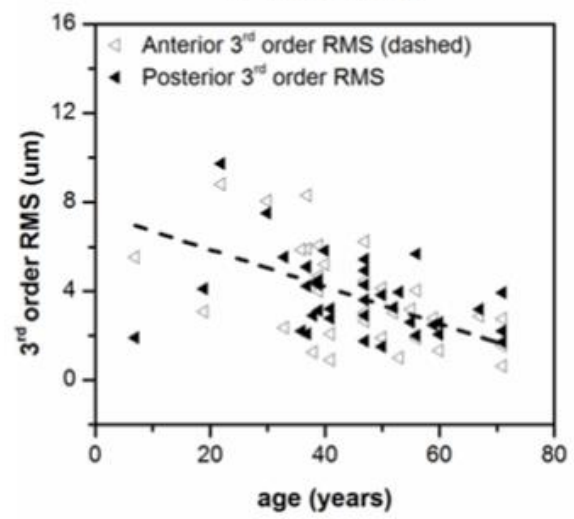

(c)

Figure 4.9 (a) Lens elevation high order RMS terms; RMS coma: slope $=-0.087 \mu \mathrm{m} / \mathrm{year}, \mathrm{r}=$ 0.582 and $\mathrm{p}=0.0001$ for anterior surface, slope $=-0.123 \mu \mathrm{m} /$ year, $\mathrm{r}=0.515$ and $\mathrm{p}=0.001$ for posterior surface; (b) RMS spherical: slope $=-0.175 \mu \mathrm{m}$ /year, $\mathrm{r}=0.439, \mathrm{p}=0.007$ for anterior surface only (c) 3rd order RMS: slope $=-0.083 \mu \mathrm{m} /$ year, $r=0.564$ and $\mathrm{p}=0.0001$ for anterior surface only

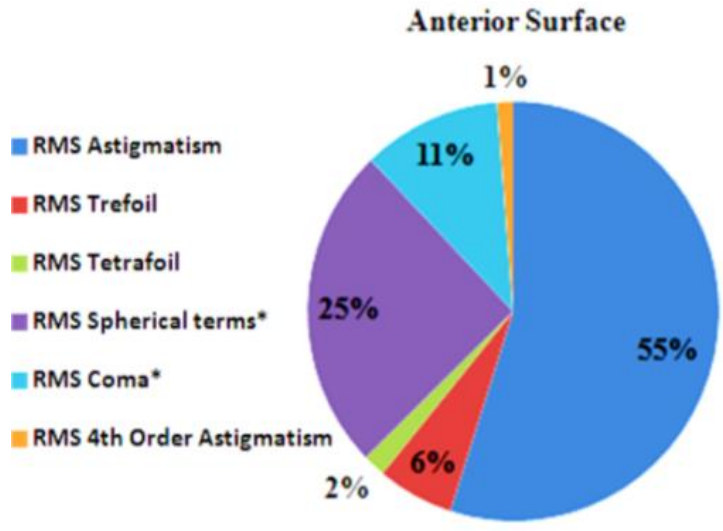

(a)

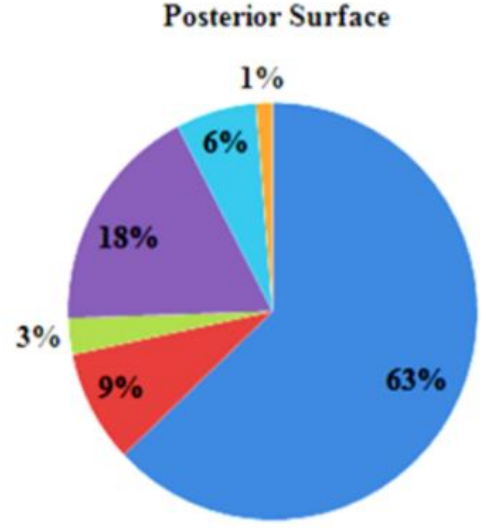

(b)

Figure 4.10 Relative contributions of different Zernike terms to the overall surface elevation maps (in terms of $\mathrm{RMS}^{2}$ ) with an asterisk the terms that change statistically significantly with age. 


\subsection{Discussion}

We have studied the 3D surface topography of 36 isolated human crystalline lenses obtained using quantitative 3D OCT. The lens surface shape has been described with radii of curvature, asphericity, and Zernike polynomials up to the $6^{\text {th }}$ order. We have evaluated the correlations between the anterior and posterior lens surface shape and their changes with age. The anterior and posterior crystalline lens surfaces have been usually described by the fitting parameters of conic functions (radius of curvature, and less frequently radius of curvature and asphericity), using measurements normally obtained from single meridians (Dubbelman and Van der Heijde 2001, Manns, Fernandez et al. 2004, Dubbelman, Van der Heijde et al. 2005). However, the direct measurements of lens surface elevations in 3D reveal that the human crystalline lens in fact shows non-spherically symmetric surfaces. Our data shows the presence of astigmatism and, to a lesser extent, other high order contributions (trefoil, spherical, coma and tetrafoil). Previous studies comparing corneal and total astigmatism predicted the presence of astigmatism in the lens, which in many cases, has a compensatory effect for corneal astigmatism (Artal, Guirao et al. 2001, Kelly, Mihashi et al. 2004).

This chapter shows that both the anterior and posterior lens surfaces contribute to lens astigmatism. The surface astigmatic power in our lens samples ranged from 0.046 to 1.185 $\mathrm{D}$ in the anterior lens surface, and from 0.013 to $1.118 \mathrm{D}$ in the posterior lens surface. However, the presence of a GRIN distribution may play a role in the overall astigmatism of the lens. Whether GRIN has a compensatory role in lens astigmatism remains to be investigated. OCT-based measurements of lens GRIN in multiple meridians in isolated lenses (Birkenfeld, de Castro et al. 2014) will help to get insights on the contribution of GRIN to astigmatism. We can only speculate on the factors contributing to lobular Zernike surface terms such as trefoil and tetrafoil, which might be related to suture branching. In a work by Gargallo et al. (Gargallo, Arines et al. 2013), lens aberrations in several species with Y-suture branches were analyzed for their relationship with suture distribution. A high degree of correlation between suture orientation and the axis of no rotationally symmetric wavefront aberrations was found. In keeping with prior studies, we found changes in lens shape with aging for both anterior and posterior lens surface. The increased steepness and negative asphericity in isolated young lenses is consistent with the fact that isolated lenses are maximally accommodated. The flattening (increased radius of curvature) and rounding (shift of asphericity towards less negative values) with age of the anterior lens surface is consistent with other studies in the literature using alternative techniques (Glasser and Campbell 1999, Dubbelman and Van der Heijde 2001, Dubbelman, Van der Heijde et al. 2005, Borja, Manns et al. 2008).

We did not find a strong correlation between surface astigmatism and age, but there were statistically significant correlations in the anterior lens surface for RMS $3^{\text {rd }}$ and RMS $4^{\text {th }}$ order Zernike terms, RMS spherical, and RMS coma $(\mathrm{p}<0.008)$, which all decreased with age. No significant correlation was found with age for surface tetrafoil and high order lobular terms, in general. Although, if lobular terms are associated with the presence of lens sutures, we would have expected an increase of the higher order terms with age, as the number of suture branches increases with aging. However, it has been shown that the formation of new branching structures is highly retarded at older ages (Kuszak, Zoltoski et 
al. 2004). Our OCT data did not provide a three-dimensional view of the lens branching, although more refined imaging modalities revealing these structures could allow modeling of the potential relationships between branching and trefoil and tetrafoil terms.

To our knowledge, this is the first comprehensive topographic study of the human lens with aging. A previous study from our laboratory showed the ability to measure the crystalline lens anterior and posterior surface elevation maps in vivo using SOCT, and demonstrated it on three young human subjects in vivo. Besides the fully accommodated state of the isolated lens and potential changes post-mortem, there may be other differences between in vivo and ex vivo conditions. For example, the zonular tension in the un-accommodated condition may cause further high order contributions to lens surface elevation in vivo (besides the flattening of the lens). Also, the up-right orientation of the lens in vivo (as opposed to the horizontal one in our ex vivo measurements) may create some changes associated to gravity.

Two of the three eyes measured in vivo in earlier studies showed perpendicular astigmatism axes in the anterior and posterior lens, whereas the astigmatic axes in this chapter tended to differ on average by $36.46 \mathrm{deg}$. In 13 of the 36 lenses, the relative angle was $>45 \mathrm{deg}$, and 6 lenses had a relative angle $>70 \mathrm{deg}$. The average relative angle was larger for younger lenses ( $41.74 \mathrm{deg}$ for lenses $<50$ years) than for older lenses ( $28.1 \mathrm{deg}$ for lenses $\geq 50$ years), indicating a higher compensation of astigmatism between the lens surfaces at a younger age.

Finally, the lack of a reference for the lenses ex vivo prevented analysis of the relative axis of astigmatism with corneal astigmatism (and therefore evaluation of potential compensatory effects between the surfaces of the cornea and lens and their changes with aging) and possible changes in the anterior lens surface astigmatic axis with aging. For example, it is well known that corneal astigmatism changes from with-the-rule to againstthe-rule astigmatism with aging (Hayashi, Masumoto et al. 1993, Gudmundsdottir, Jonasson et al. 2000, Ho, Liou et al. 2010). Previous studies suggested that the astigmatism axis of the anterior lens surface is vertical (Alvarez, Turner et al. 2003, Koch, Ali et al. 2012, Ortiz, Perez-Merino et al. 2012). We did not find a significant correlation between anterior or posterior astigmatism axis and age. However, the tendency of the relative angle to decrease with age could be interpreted as a decrease of the anterior by posterior astigmatism balance with age.

Regardless of potential information provided by future in vivo studies of lens surface topography and its changes with age, this chapter shows that astigmatism and high order terms may be considered when trying to predict optical quality of a phakic eye at an individual level, based on anatomical information. This information could be used in a ray tracing analysis that incorporates data from cornea and lens GRIN distribution (Manns, Fernandez et al. 2004, Marcos, Rosales et al. 2007, Acosta, Bueno et al. 2010, Ortiz and Perez-Merino et al. 2012).In the following chapter, we will use geometrical and anatomical information (including corneal and lens shape and lens tilt) to generate custom computer eye models, which save as platform to understand the relative contributions of each factor to optical quality and optimize lens selection. 


\section{Chapter 5}

\section{FULL 3D OCT-BASED PSEUDOPHAKIC CUSTOM COMPUTER EYE MODEL}


This chapter is based on the following publication:

M. Sun, P. Pérez-Merino, E. Martinez-Enriquez, M. Velasco-Ocana and S. Marcos (2016). "Full 3-D OCT-based pseudophakic custom computer eye model." Biomedical Optics Express 7(3): 1074-1088.

The contribution of Mengchan Sun to the study, in collaboration with other coauthors, was the literature search, the design of the experiments and the data acquisition (in collaboration with Pablo Pérez-Merino), the development of specific routines and processing of the data (in collaboration with Edurado Martinez-Enriquez), and the building modeling and analysis the data. This work was also presented at the 2014 Visual and Physiological Optics conference (VPO) in Wroclaw (Poland). 


\section{RESUMEN}

Objetivos: Además de la medida y descripción de las aberraciones oculares, es particularmente importante la comprensión de las fuentes de aberraciones ópticas a nivel individual, en particular dado que minimizan la inducción de aberraciones en el tratamiento quirúrgico o óptico. El modelo de ojo completamente personalizado basado en los datos geométricos OCT 3D en un ojo implantado con IOL permitirá investigar la contribución relativa del diseño de IOL y otros factores a la calidad óptica. Además, los ojos de los modelos basados en OCT prometen convertirse en la referencia para la selección de potencia de la IOL basada en el trazado de rayos (preoperatorio) y para la comprensión de los mecanismos operativos y los resultados funcionales de las IOLs de alta calidad.

Métodos: Tres ojos de dos pacientes (64 y 78 años) implantados con IOLs se midieron tres meses después de la cirugía de cataratas. Los datos de elevación basados en OCT de las superficies corneales anteriores y posteriores, las distancias del segmento anterior, la geometría de la IOL y los datos de alineación, el ángulo lambda (todos procedentes de sOCT) y la longitud axial del IOLMaster se exportaron a ZEMAX, donde se construyeron los ojos de los modelos personalizados. Las aberraciones de onda se calcularon en el plano de la pupila mediante el trazado en una matriz de $64 \times 64$ rayos colimados a través de las superficies oculares dentro de un área central de $5 \mathrm{~mm}$ de diámetro de la pupila, a $555 \mathrm{~nm}$. Los datos se analizaron en términos de términos de Zernike y la métrica de calidad de imagen retinal Visual Strehl). Se compararon las aberraciones de onda medida medidas usando un sistema de trazado de rayos por láser en ojos pseudofáquicos implantados con lentes intraoculares asféricas (IOLs) con aberraciones simuladas de trazado de rayos numéricos en modelos de ojo computacionales personalizados, construidos utilizando la geometría ocular cuantitativa 3-D basada en OCT.

Resultados: Existe una correlación altamente estadísticamente significativa entre los coeficientes de Zernike medidos por LRT y los simulados a partir de modelos computacionales que incorporan todos los parámetros geométricos medidos (coeficiente de correlación medio $\mathrm{R}=0.93 \pm 0.05 \mathrm{y} \mathrm{p}<0.0001$ ). Las correlaciones, aunque estadísticamente significativas, son menores entre coeficientes de Zernike de las aberraciones totales y de las aberraciones de la superficie anterior de la córnea (coeficiente de correlación promedio $\mathrm{R}=0.73 \pm 0.05$ y $\mathrm{p}<0.0001$ ), seguido de las aberraciones de toda la córnea (anterior+posterior) (coeficiente de correlación medio $\mathrm{R}=0.88 \pm 0.03, \mathrm{p}<0.0001$ ); y muy cercano a 1 entre las aberraciones totales medidas y las simuladas incluyendo cornea, IOL $\mathrm{y}$ alineamiento (coeficiente de correlación medio $\mathrm{R}=0.94 \pm 0.04$ y $\mathrm{p}<0.0001$ ). La discrepancia entre las aberraciones medidas y reales es inferior al $14.33 \%(0.15 \mu \mathrm{m})$ para el astigmatismo, y menos del $21.43 \%(0.03 \mu \mathrm{m})$ para la aberración esférica. En promedio, las discrepancias para RMS para astigmatismo y aberraciones de orden superior y RMS para aberraciones de alto orden son sólo $0.12 \mu \mathrm{m}$ y $0.08 \mu \mathrm{m}$, respectivamente.

Conclusiones: Este estudio muestra que los modelos de ojo computacionales pseudofáquicos basados en OCT permiten entender la contribución relativa de los factores ópticos geométricos y quirúrgicos a la calidad de la imagen y son una excelente herramienta para caracterizar y mejorar la cirugía de cataratas. 


\subsection{Introduction}

Ocular aberrometry (introduced in Section 1.4.3) has become a standard tool in research and in the clinic to measure the optical quality of the eye, and its changes with aging (McLellan, Marcos et al. 2001), and accommodation (Atchison, Collins et al. 1995, He, Burns et al. 2000). In addition, ocular aberrometry is useful in surgical interventions (i.e. LASIK (Moreno-Barriuso, Merayo-Lloves et al. 2001), intraocular lens (IOL) implantation surgery (Barbero, Marcos et al. 2003, Marcos, Barbero et al. 2005), keratoconus and Intracorneal Ring Segment (ICRS) implantation (Perez-Merino, Ortiz et al. 2014), etc...). Furthermore, new refractive products and, more specifically, the availability of a large number of IOL options hold promise for a customized intervention, adapted to the patient's needs. There is an increasing number of available IOLs aiming at modifying the patient's wave aberrations, targeting correction of both refractive error and some high order aberrations (i.e. spherical aberration, such as in aspheric designs, or aiming at the increase of depth-of-focus in presbyopic-correction IOLs). However, these efforts have not in general been paralleled by a sophistication of the methods to select the most suitable IOL, or to predict the optical quality outcomes. Generally, the methods to calculate the power of the IOL to be implanted are based on paraxial regression formulae, based on population averages (Olsen 2007). Furthermore, simulations of the optical performance of new IOL designs either generally assume diffraction-limited optics in the eye, or simple eye models (Holladay, Piers et al. 2002) that are based on average anatomic data across the population and numerous simplifications (i.e. rotationally centered optics, symmetric optical surfaces, etc...). The suitability of custom eye models to improve the predictability of the results of cataract surgery has been acknowledged. Several authors have presented the use of nonparaxial eye models for improved selection of IOL to be implanted, in particular for the calculation of IOL power with higher accuracy than regression-formulae (Hoffmann, Wahl et al. 2012, Minami, Kataoka et al. 2012, Olsen and Hoffmann 2014, Saiki, Negishi et al. 2014). Customized eye models have also been shown to accurately reproduce measured high order aberrations, when constructed using the anatomical parameters of individual patients (Tabernero, Piers et al. 2006, Rosales and Marcos 2007). These models are instrumental in understanding the relative contribution of the patient's corneal topography, IOL design, IOL tilt and decentration, and foveal misalignment, and as well as platforms to test what optical performance would have resulted if the eye had been implanted with a different IOL design. Typically, the anatomical parameters of the eye used to build these eye models are obtained from various instruments (Placido Disk, corneal elevation maps; Purkinje imaging IOL tilt, decentration and foveal position; IOL design provided by the manufacturer) (Tabernero, Piers et al. 2006, Rosales and Marcos 2007). Recently, quantitative OCT imaging has shown to be a powerful technology to obtain quantitative 3D optical biometry and geometry in individual eyes (Ortiz, Perez-Merino et al. 2013), providing, from a single instrument, all the ocular anterior segment information needed to build a custom pseudophakic model eye.

The quantitative OCT anterior segment imaging technique has been demonstrated to provide accurate corneal topographies, crystalline lens topographies as we described in Section 2.1.2 (Quantification tools), 3D optical biometry pre- and post-operative cataract surgery (Ortiz, Perez-Merino et al. 2013) and tilt and decentration of IOL and/or crystalline lens by Purkinje-like OCT en-face reflection image described in Section 2.1.3. In addition, 
previous work has shown that ray tracing through OCT-based corneal topographies allows computation of corneal aberrometry, showing high degrees of agreement with total aberrometry in a group of keratoconic eyes pre- and post- implantation of intrastromal corneal ring segments (Perez-Merino, Ortiz et al. 2014). Also, 3D biometric quantitative anterior segment OCT in patients with accommodating IOLs (Marcos, Ortiz et al. 2014) shows good correspondence with certain aberration terms measured on the same eyes: i.e. the observed axial shifts with accommodative demand are in good agreement with the measured shifts in defocus, or the increased amounts of IOL tilt with the measured increased coma (Marcos, Ortiz et al. 2014).

In this chapter we present a customized model eye fully based on 3D geometrical OCT data in patients implanted with IOLs. Comparison of the geometry of IOL ex vivo samples to the IOL geometry measured in vivo will serve as further support of the quantitative potential of the 3D anterior segment OCT. Experimental measurements of ocular aberrations on these eyes will be compared with estimates of ocular aberrations from numerical ray tracing using a customized model eye from individual OCT measurements of post-operative corneal topography, anterior chamber depth, IOL geometry, IOL tilt and decentration and foveal misalignment. This study will allow validation of the accuracy of OCT-based customized eye models to predict measured aberrations, and to investigate the contribution of the different components to overall image quality in eyes with IOLs, thus demonstrating the capability of quantitative 3D anterior segment OCT to assess postoperative optical quality in pseudophakic eyes. These validations will be a first step into investigating the potential for OCT-based model eyes to become the reference for ray tracing based IOL selection (pre-operatively) and to understand mechanisms of operation and optical outcomes of premium IOLs. 


\subsection{Methods}

\subsubsection{Patients, surgery and measurements}

Three eyes (2, OS; 1, OD) of two patients (64 and 78 years old) implanted with IOLs from two different manufacturers (Akreos AO M160, Bausch and Lomb, Rochester, New York, US; CT Asphina 409M, Zeiss, Jena, Germany) were measured three months after cataract surgery. Standard phaco-emulsification intracapsular cataract surgery had been performed by a single surgeon at Fundación Jiménez Díaz Hospital (FJD) with 2-mm corneal incisions (temporal in OD and nasal in OS). Table 5.1 shows the patients' clinical profile and details on the implanted IOLs. Total wave aberrations were measured using a custom-developed Laser Ray Tracing (LRT) system (Llorente, Barbero et al. 2004). Anterior segment geometry (cornea, iris and IOL) was measured using a custom-developed spectral OCT (sOCT) (Grulkowski, Gora et al. 2009). Measurements were done under mydriasis $(2.5 \%$ phenylephrine). LRT measurements were done under foveal fixation, and OCT measurements were done at the off-axis fixation that aligns the pupillary axis with the optical axis of the instrument. Experimental protocols were approved by Institutional Review Boards at FJD and CSIC. Patients signed informed consent after the nature of the study had been explained to them. All protocols followed the tenets of the Declaration of Helsinki.

Table 5.1 Patients' clinical profile and details on the implanted IOLs

\begin{tabular}{ccccccccc}
\hline Subject & $\begin{array}{c}\text { Male/ } \\
\text { Female }\end{array}$ & Age & Lens implanted & $\begin{array}{c}\text { ACD } \\
(\mathbf{m m})\end{array}$ & $\begin{array}{c}\text { AL } \\
(\mathbf{m m})\end{array}$ & $\begin{array}{c}\text { IOL } \\
\text { Power }\end{array}$ & $\begin{array}{c}\text { Post-op } \\
\text { Spherical Error }\end{array}$ & $\begin{array}{c}\text { Post-op } \\
\text { Cylinder/axis }\end{array}$ \\
\hline S1-OD & Male & 78 & Akreos,Bausch+Lomb & $4.31 \pm 0.02$ & 23.35 & $20 D$ & -0.50 & $-1.75 / 100$ \\
S2-OS & Female & 64 & Asphina IOL,Zeiss & $3.80 \pm 0.01$ & 23.08 & $20.5 D$ & +0.25 & $-1.00 / 85$ \\
S3-OS & Male & 78 & Akreos,Bausch+Lomb & $4.48 \pm 0.02$ & 23.28 & $21.5 D$ & +1.25 & $-1.25 / 90$ \\
\hline
\end{tabular}

\subsubsection{D anterior segment OCT}

The sOCT system used in the measurements has been described in detail in Section 2.1.1. The effective acquisition speed is $25000 \mathrm{~A}-\mathrm{Scans} / \mathrm{s}$. The axial resolution given by the coherence length of the OCT is $6.9 \mu \mathrm{m}$. The axial range is $7 \mathrm{~mm}$ in depth, resulting in a theoretical pixel resolution of $3.4 \mu \mathrm{m}$. Measurements were collected in a $10 \times 10 \mathrm{~mm}$ area, and consisted of a collection of $50 \mathrm{~B}$-scans composed by $300 \mathrm{~A}$-scans. In this study, we consecutively measured the cornea with decreasing acquisition times (from $2.0 \mathrm{~s}$ to $0.5 \mathrm{~s}$ ) and found that measurements below 0.8 seconds were stable, preventing the need for distortion artifacts that we developed for that matter, in most cases (Ortiz, Siedlecki et al. 2011). In this study, we used a $0.6 \mathrm{~s}$ acquisition speed in a dense, regular and homogeneous sampling which was deemed to provide an appropriate balance between resolution and speed for repetitive measurements and did not require the implementation of motion compensation by software (Ortiz, Siedlecki et al. 2011).

Measurements were obtained while the subject fixates on a minidisplay that projects a 20/20 Snellen E-letter stimulus. The stimulus is moved across the display to change the 
eye's fixation angle, so that the optical axis of the instrument is aligned with the pupillary axis (Perez-Merino, Velasco-Ocana et al. 2015) in Figure 5.1 (a). For foveal fixation the iris appears asymmetrically tilted (Figure 5.1(b)), whereas for the selected fixation the iris appears flat (Figure 5.1(c)) in the continuous horizontal and vertical cross-sectional views of the anterior segment used during alignment. The off-axis position of the fovea (angle lambda, $\lambda$ ) is calculated from the eye rotation between both alignments. Sets of 3D images of the anterior segment were captured approximately 5 seconds after blinking.

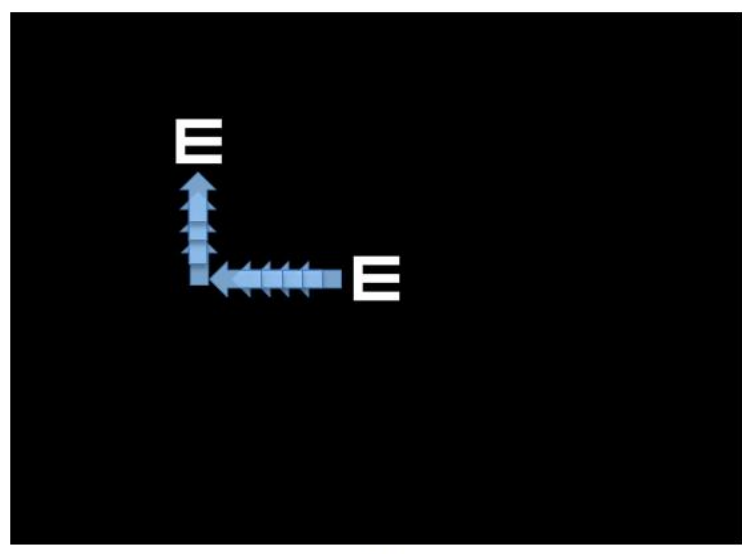

(a)

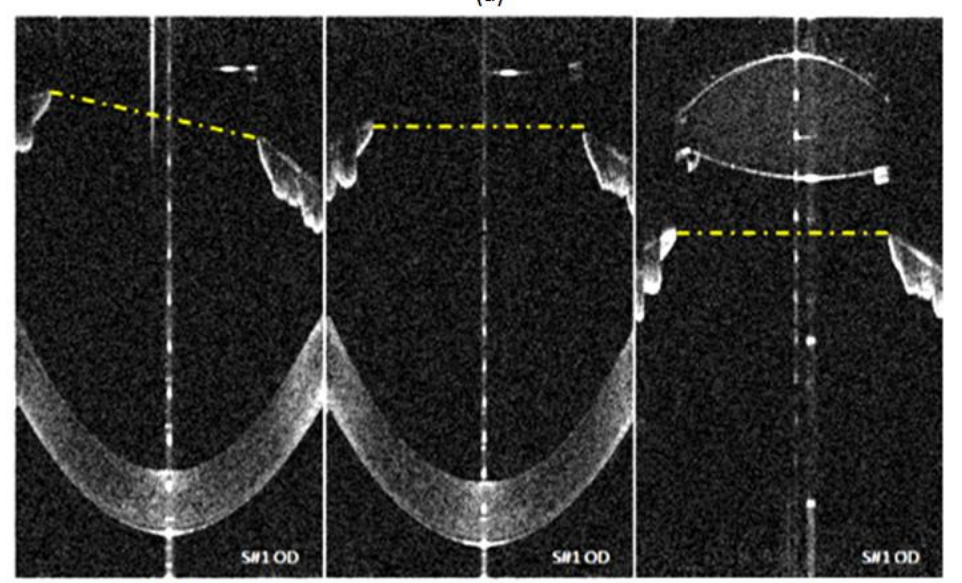

(b)

(c)

(d)

Figure 5.1 (a) Example movement of 20/20 Snellen E-letter across the display to change the eye's fixation angle stimulus; Acquisition at two different foci (cornea and lens) (b) Vertical crosssectional scans of a patient's cornea while fixating foveally, i.e. along the line of sight; (c) Vertical cross-sectional scans of a patient's cornea, while fixating eccentrically, i.e. pupilary axis aligned with the OCT optical axis; (d) Vertical cross-sectional scans of the IOL implanted in the patient. Typical image acquisitions are obtained with the alignment as in (c) and (d). The yellow line indicates the iris plane, used for merging.

The automatic image processing tools for denoising, segmentation, clustering, merging, and biometric and IOL alignment measurements have been described in detail before in Section 2.6.2 (MATLAB routines). The quantification capabilities of the instrument have been demonstrated with artificial model eyes with known dimensions, ex vivo and in vivo measurements, and comparisons with other instruments (Scheimpflug, Purkinje imaging, and non-contact profilometry) in Section 2.3 (Other techniques for validation).

Anterior and posterior corneal elevation maps, corneal thickness, and anterior chamber depth were obtained from 3D OCT as described in earlier Section 2.1.2 (Quantification 
tools). Anterior and posterior corneal elevation maps were fit by $6^{\text {th }}$ order Zernike polynomials. For comparison, anterior and posterior corneal topographies were also obtained from the Pentacam Scheimpflug topographer (Oculus, Inc., Lynnwood, WA) maintained in Section 2.3.1. IOL tilt and decentration was obtained using the OCT-based method described in Section 2.1.3.

\subsubsection{Ex vivo IOL geometry: non-contact profilometry}

The geometry of the IOLs (Akreos 20D, 21.5D, Bausch+Lomb; Asphina 20.5D, Zeiss) was characterized ex vivo using microscopy-based noncontact profilometry (Sensofar, PLu2300, Barcelona, Spain) with 0.1- $\mu$ m nominal vertical precision (on rigid samples) as maintained in Section 2.3.2. De-hydration effects on these IOLs with high water content (25-26\%) decrease the effective precision. Briefly, repeated measurements on these lenses revealed repeatability in the apex measurement of $5 \mu \mathrm{m}$. Both anterior and posterior lens surface topographies were measured in a $5.5 \times 5.5 \mathrm{~mm}$ range ( $50 \times 50$ points, $0.11 \mathrm{~mm}$ steps). The IOL surface profiles were fitted by conics with MATLAB algorithms.

\subsubsection{Customized eye models}

In Section 2.3.4, we introduced the customized computer eye models. As a brief summary, OCT-based elevation data from both anterior and posterior corneal surfaces, anterior segment distances, IOL geometry and alignment data, angle lambda (all coming from sOCT), and axial length from the IOLMaster (Carl Zeiss AG, Jena, Germany) were exported to ZEMAX (Radiant ZEMAX; Focus software, Tucson, AZ, USA), where the custom model eyes were built. Refractive indices of the cornea, aqueous humour, vitreous humour, Akreos IOLs and Asphina IOLs were 1.376, 1.337, 1.337, 1.458 and 1.460 respectively, at $555 \mathrm{~nm}$. Wave aberrations were calculated in the pupil plane by tracing an array of $64 \times 64$ collimated rays through the eye surfaces within a central 5-mm pupil diameter area, at $555 \mathrm{~nm}$. Figure 2.15 illustrates the computation of aberrations from OCT data, i.e. ray tracing calculation on OCT distortion-corrected corneal and IOL surfaces. Data are analyzed in terms of Zernike terms and retinal image quality metrics (MTF and Visual Strehl (Iskander 2006)).

\subsubsection{Total aberration measurements: LRT}

Total wave aberrations were measured using custom LRT, described in detail in Section 2.2. In brief, an infrared (785-nm) laser beam sampled 37 positions across the pupil sequentially in a hexagonal sampling pattern while a charge couple device (CCD) camera recorded the corresponding aerial images of light reflected off the retina. The sampling pattern was adjusted by software to fit a 5-mm pupil centered at the pupil center. Prior to the measurement, the patient adjusted his/her subjective refraction using a Badal optometer while viewing a stimulus on a minidisplay placed on a retinal conjugate plane. Ray 
aberrations were obtained by estimating the deviations of the centroids of the retinal spots images corresponding to each pupil entry location with respect to the reference (chief ray). These deviations are proportional to the local derivatives of the wave aberrations. Maximum energy exposure was $6.8 \mu \mathrm{W}$. All measurements were done under foveal fixation of a Maltese cross. Wave aberrations were fitted by $6^{\text {th }}$ order Zernike polynomial expansions, with ordering and normalization following OSA standards (Thibos, Applegate et al. 2002), and were described in terms of individual Zernike coefficients or RMS, which was used to report the magnitude of HOA. Computations of the Modulation Transfer Function (MTF) from the wave aberrations were performed using standard Fourier Optics. RMS wavefront error and the MTF were used as optical quality metrics (More detail can be found in Section 2.4.2 and 2.4.3). 


\subsection{Results}

\subsubsection{OCT-based anterior segment geometry in pseudophakic eyes}

Table 5.2 shows the anterior and posterior corneal shape (radii of curvature calculated from the best-fit sphere over 6-mm diameter and some relevant Zernike terms from the polynomial fitting to the corneal elevation maps) and biometry (3D OCT Corneal thickness, and Anterior Chamber Depth).

Table 5.2 Individual OCT-based biometrical and geometrical data used in the computer model eyes. Data are shown as average \pm standard deviation of repeated measurements

\begin{tabular}{|c|c|c|c|}
\hline & S\#1 OD & S\#2 OS & S\#3 OS \\
\hline Anterior corneal radius (mm) ${ }^{*}$ & $7.74 \pm 0.16$ & $7.67 \pm 0.19$ & $7.77 \pm 0.18$ \\
\hline \multirow{7}{*}{$\begin{array}{c}\text { Anterior corneal elevation } \\
\text { selected Zernike values }(\mu \mathrm{m})^{*} \text { : }\end{array}$} & $Z_{2}^{2}: 119.6 \pm 58.9$ & $Z_{2}^{2}: 120.5 \pm 60.5$ & $Z_{2}^{2}: 118.4 \pm 59.2$ \\
\hline & $Z_{2}^{-2}: 1.48 \pm 0.05$ & $Z_{2}^{-2}: 1.10 \pm 0.14$ & $Z_{2}^{-2}: 1.35 \pm 0.33$ \\
\hline & $Z_{3}^{1}: 0.21 \pm 0.07$ & $Z_{3}^{1}: \quad 0.42 \pm 0.69$ & $Z_{3}^{1}: 0.13 \pm 0.35$ \\
\hline & $Z_{3}^{-1}:-0.33 \pm 0.68$ & $Z_{3}^{-1}: 0.40 \pm 0.11$ & $Z_{3}^{-1}:-0.12 \pm 0.19$ \\
\hline & $Z_{3}^{3}:-0.36 \pm 0.19$ & $Z_{3}^{3}: \quad-0.37 \pm 0.18$ & $Z_{3}^{3}:-0.13 \pm 0.01$ \\
\hline & $Z_{3}^{-3}:-0.04 \pm 0.12$ & $Z_{3}^{-3}:-0.71 \pm 0.36$ & $Z_{3}^{-3}: 0.13 \pm 0.13$ \\
\hline & $Z_{4}^{0}: 0.93 \pm 0.88$ & $Z_{4}^{0}: \quad 0.74 \pm 0.32$ & $Z_{4}^{0}: 0.65 \pm 0.97$ \\
\hline Posterior corneal radius (mm)* & $6.28 \pm 0.14$ & $6.18 \pm 0.13$ & $6.36 \pm 0.13$ \\
\hline \multirow{7}{*}{$\begin{array}{c}\text { Posterior corneal elevation } \\
\text { selected Zernike values }(\mu \mathrm{m})^{*} \text { : }\end{array}$} & $Z_{2}^{2}: 147.2 \pm 71.2$ & $Z_{2}^{2}: 151.6 \pm 77.9$ & $Z_{2}^{2}: 147.2 \pm 73.6$ \\
\hline & $Z_{2}^{-2}:^{-10.85 \pm 1.33}$ & $Z_{2}^{-2}:-5.42 \pm 2.04$ & $Z_{2}^{-2}:-9.80 \pm 0.01$ \\
\hline & $Z_{3}^{1}:-0.13 \pm 0.59$ & $Z_{3}^{1}: 0.21 \pm 0.60$ & $Z_{3}^{1}: 0.46 \pm 0.97$ \\
\hline & $Z_{3}^{-1}: 0.36 \pm 1.31$ & $Z_{3}^{-1}: 1.00 \pm 1.19$ & $Z_{3}^{-1}:-0.66 \pm 1.64$ \\
\hline & $Z_{3}^{3}:-0.30 \pm 0.50$ & $Z_{3}^{3}: \quad 0.22 \pm 0.23$ & $Z_{3}^{3}: \quad-0.01 \pm 0.81$ \\
\hline & $Z_{3}^{-3}: 0.40 \pm 0.67$ & $Z_{3}^{-3}: \quad-0.64 \pm 0.13$ & $Z_{3}^{-3}:-1.20 \pm 0.24$ \\
\hline & $Z_{4}^{0}: 1.00 \pm 0.07$ & $Z_{4}^{0}: \quad 2.80 \pm 1.32$ & $Z_{4}^{\circ}: \quad 0.70 \pm 0.59$ \\
\hline Corneal Thickness (mm) & $0.53 \pm 0.01$ & $0.57 \pm 0.01$ & $0.53 \pm 0.01$ \\
\hline $\operatorname{ACD}(\mathbf{m m})$ & $4.31 \pm 0.02$ & $3.80 \pm 0.01$ & $4.48 \pm 0.02$ \\
\hline IOL thickness (mm) & $0.91 \pm 0.01$ & $1.01 \pm 0.01$ & $0.85 \pm 0.05$ \\
\hline
\end{tabular}

* from the corresponding best-fitting sphere

Figure 5.2 shows OCT-based anterior and posterior corneal elevation maps in all 3 eyes, centered at the pupil center, and for 6-mm pupil diameters. For comparison, corneal maps from Pentacam obtained on the same eyes using the same pupil reference and analysis are also shown. The RMS difference in corneal elevation between instruments is less than 0.04 $\mathrm{mm}$ and $0.06 \mathrm{~mm}$, for anterior and posterior surfaces, respectively. In all eyes, astigmatism appears to be the dominant term in the posterior corneal maps, with $19.6 \%$ average 
difference in magnitude and $6.5 \mathrm{deg}$ difference in axis between OCT and Pentacam topographies.

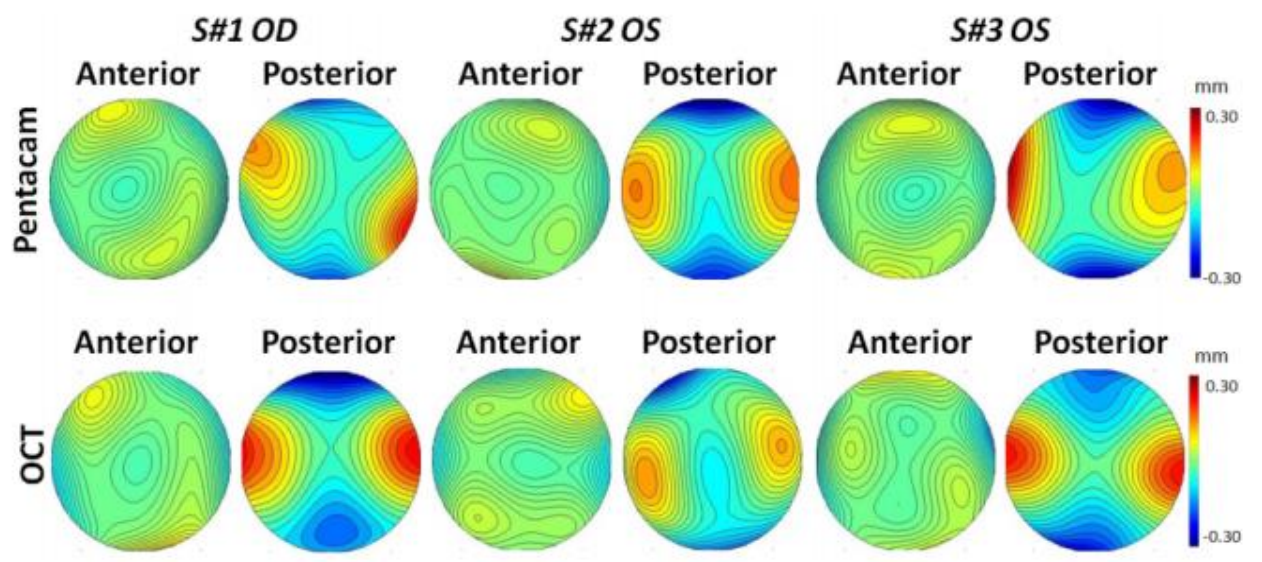

Figure 5.2 Anterior and posterior corneal topographic maps from Pentacam (upper panel) and OCT (lower panel). OCT-based maps were used in the computer eye models. Data are for 6-mm pupil.

Table 5.3 shows measurements of eye rotation and IOL tilt and decentration (horizontal and vertical components) for each eye. The tilt and decentration nomenclature and sign conventions correspond to those published in prior publications (for an illustration of the convention see Figure 5.6 in Rosales et al. 2008 (Rosales, Wendt et al. 2008)).

Table 5.3 Eye rotation and IOL tilt and decentration data from OCT-based Purkinje-like methods. Data are average \pm standard deviation of 5 repeated measurements. Rotation/tilt $\mathrm{X}$ stands for rotation/tilt around the horizontal axis; Rotation/tilt Y stands for rotation/tilt around the vertical axis. Decentration X/Y stands for horizontal /vertical decentration.

\begin{tabular}{cccc}
\hline Visual axis & S\#1 OD & S\#2 OS & S\#3 OS \\
\hline $\begin{array}{c}\text { Eye rotation } \boldsymbol{X} \\
\text { (degree) }\end{array}$ & $-1.33 \pm 0.46$ & $-1.83 \pm 0.38$ & $-0.14 \pm 0.27$ \\
$\begin{array}{c}\text { Eye rotation } \boldsymbol{Y} \\
\quad \text { (degree) }\end{array}$ & $4.13 \pm 0.21$ & $-3.55 \pm 0.58$ & $-2.43 \pm 0.78$ \\
$\begin{array}{c}\text { IOL tilt } \boldsymbol{X}(\text { degree }) \\
\text { IOL tilt } \boldsymbol{Y} \text { (degree) }\end{array}$ & $-2.22 \pm 0.49$ & $1.07 \pm 0.41$ & $-2.46 \pm 0.26$ \\
$\begin{array}{c}\text { IOL decentration } \boldsymbol{X} \\
(\text { mm) }\end{array}$ & $0.01 \pm 0.06$ & $-0.61 \pm 0.05$ & $-1.20 \pm 0.35$ \\
$\begin{array}{c}\text { IOL decentration } \boldsymbol{Y} \\
(\text { mm })\end{array}$ & $-0.12 \pm 0.19$ & $0.19 \pm 0.16$ & $-0.32 \pm 0.14$ \\
\hline
\end{tabular}


Full 3D OCT-based pseudophakic custom computer eye model

\subsubsection{Comparing OCT-based IOL geometry in vivo with profilometry ex vivo}

Comparison of the IOL geometry measured in vivo (OCT) and ex vivo (non-contact profilometry) serves as a further validation of the accuracy of OCT (provided with distortion correction algorithms and analysis tools). Table 5.4 compares the radii of curvature and asphericity values for anterior and posterior IOL surfaces from profilometry and OCT, and the estimated IOL power from these values, compared to the nominal IOL power. The mean differences between the radii of curvature measured by profilometry ex vivo and OCT in vivo are $0.40 \pm 0.28 \mathrm{~mm}$ (96.83\% agreement), and for the asphericity 2.17 $\pm 1.64(17.96 \%)$. The geometrical differences between techniques result in less than 0.44 D difference between the IOL power estimated from profilometry and OCT, and less than $0.22 \mathrm{D}$ between the OCT based IOL power estimates in vivo and the nominal IOL power.

Table 5.4 Anterior and posterior radius of curvature (R), asphericity (Q) from profilometry and OCT, and the IOL power estimated from profilometry and OCT. OCT-based data were used in the computer eye models.

\begin{tabular}{cccccccc}
\hline & & \multicolumn{2}{c}{ S\#1 OD } & \multicolumn{2}{c}{ S\#2 OS } & \multicolumn{2}{c}{$S \# 3$ OS } \\
\hline \multirow{2}{*}{ Anterior } & Profilometry & $10.55 \pm 0.06$ & $-0.03 \pm 0.11$ & $11.90 \pm 0.17$ & $-0.24 \pm 0.05$ & $10.00 \pm 0.28$ & $-0.3 \pm 0.11$ \\
IOL & $O C T$ & $11.00 \pm 0.10$ & $-4.56 \pm 0.08$ & $11.51 \pm 0.11$ & $-2.63 \pm 1.62$ & $10.05 \pm 0.18$ & $-1.44 \pm 0.23$ \\
Posterior & Profilometry & $13.53 \pm 0.5$ & $-1.09 \pm 0.14$ & $12.90 \pm 1.92$ & $-0.5 \pm 0.64$ & $12.80 \pm 3.01$ & $-0.4 \pm 0.11$ \\
IOL & OCT & $14.2 \pm 0.05$ & $-0.05 \pm 0.40$ & $12.17 \pm 3.9$ & $00.8 \pm 0.41$ & $12.9 \pm 0.33$ & $-3.99 \pm 0.80$ \\
& Nominal & $20 D$ & $20.5 D$ & $21.5 D$ \\
IOL & Profilometry & $20.4 D$ & $20.8 D$ & $21.7 D$ \\
Power & OCT & $19.9 D$ & $20.8 D$ & $21.8 D$ \\
\hline
\end{tabular}

\subsubsection{Ocular aberrations: LRT vs. Computer Ray Tracing on custom model eye}

Figure 5.3 shows examples of measured (first column) and simulated (column 2-5) wave aberrations in three subjects (S\#1 OD, S\#2 OS, and S\#3 OS) for (a) $2^{\text {nd }}$ and higher order aberrations excluding tilt and defocus and (b) $3^{\text {rd }}$ and higher order aberrations, for 5- $\mathrm{mm}$ pupil diameters. Anterior cornea stands for wave aberration of the eye with only the anterior corneal surface; Cornea total stands for the wave aberration of the eye with a 2- surface cornea, without the IOL; Cornea + IOL stands for the wave aberration of the eye with the IOL assuming that the IOL is perfectly aligned with respect to the pupillary axis; Corneal + rot/dec IOL stands for the wave aberration of the eye with the IOL tilted and decentered according to the measured tilts and decentrations.

The eyes are rotated according to angle $\lambda$ in order to incorporate the line-of-sight misalignment. In general, the corneal wave aberration map shows many of the relevant features of the measured wave aberrations. 

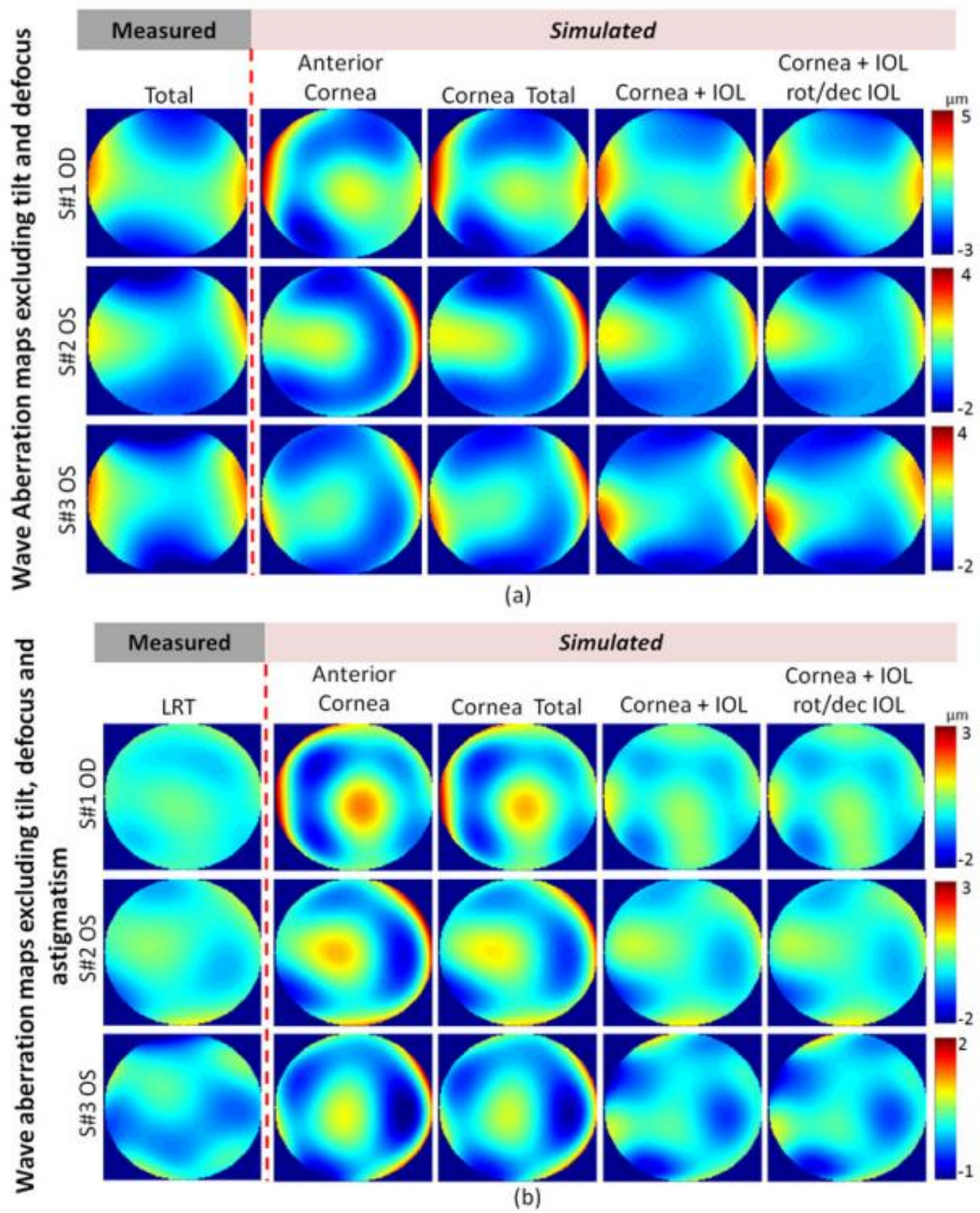

Figure 5.3 Measured (LRT, $1^{\text {st }}$ column) and Simulated (from OCT-based geometry/biometry, $2^{\text {nd }}$ $-5^{\text {th }}$ columns) wave aberration maps in three pseudophakic eyes. Simulated aberrations include wave aberration maps for the anterior cornea alone ( $2^{\text {nd }}$ column); anterior and posterior cornea ( $3^{\text {rd }}$ column); eye wave aberration with IOL assuming no tilt and decentration ( $4^{\text {th }}$ column); eye wave aberration with IOL with no tilt/decentration $\left(5^{\text {th }}\right.$ column). (a) Wave aberration maps including astigmatism (no tilt or defocus); (b) Wave aberrations for 3rd and higher order; Data are for 5-mm pupil, and for foveal fixation, i.e.including eye rotation.

Figure 5.4 compares RMS wavefront error for different Zernike orders and terms for the LRT-measured eye aberrations, for the cornea (anterior only or total cornea), for the total eye assuming that the IOL is centered and for the total eye with the real tilt and decentration with measured total aberrations. The discrepancy between the measured and real 
aberrations is less than $14.33 \%(0.15 \mu \mathrm{m})$ for astigmatism, and less than $21.43 \%(0.03 \mu \mathrm{m})$ for spherical aberration. In contrast, the larger differences occur for trefoil (in Subjects 1 and 3), coma (in Subject 3) and tetrafoil (in all Subjects). On average, the discrepancies for RMS for astigmatism and higher order aberrations and RMS for high order aberrations alone are $0.12 \mu \mathrm{m}$ and $0.08 \mu \mathrm{m}$, respectively.
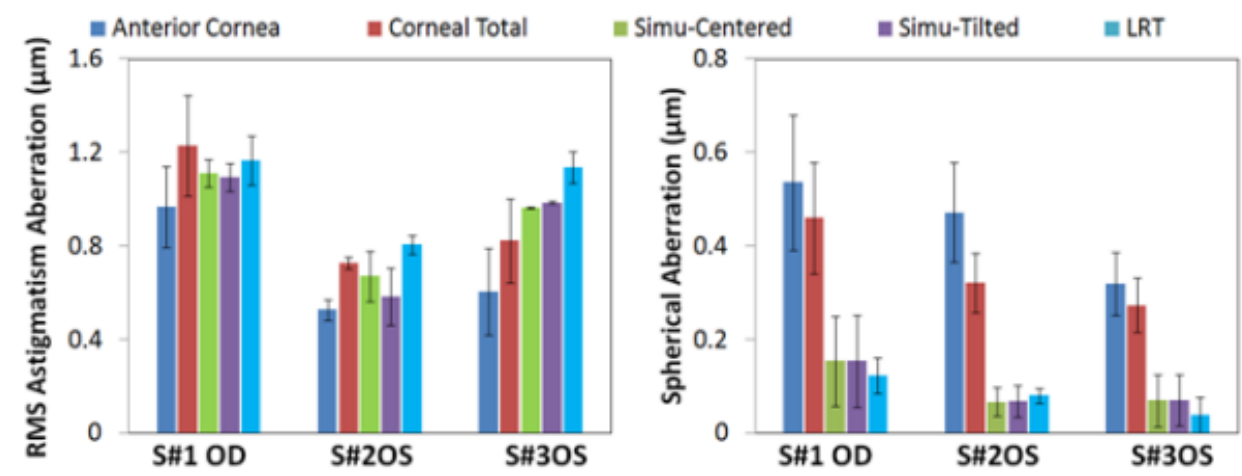

(a)
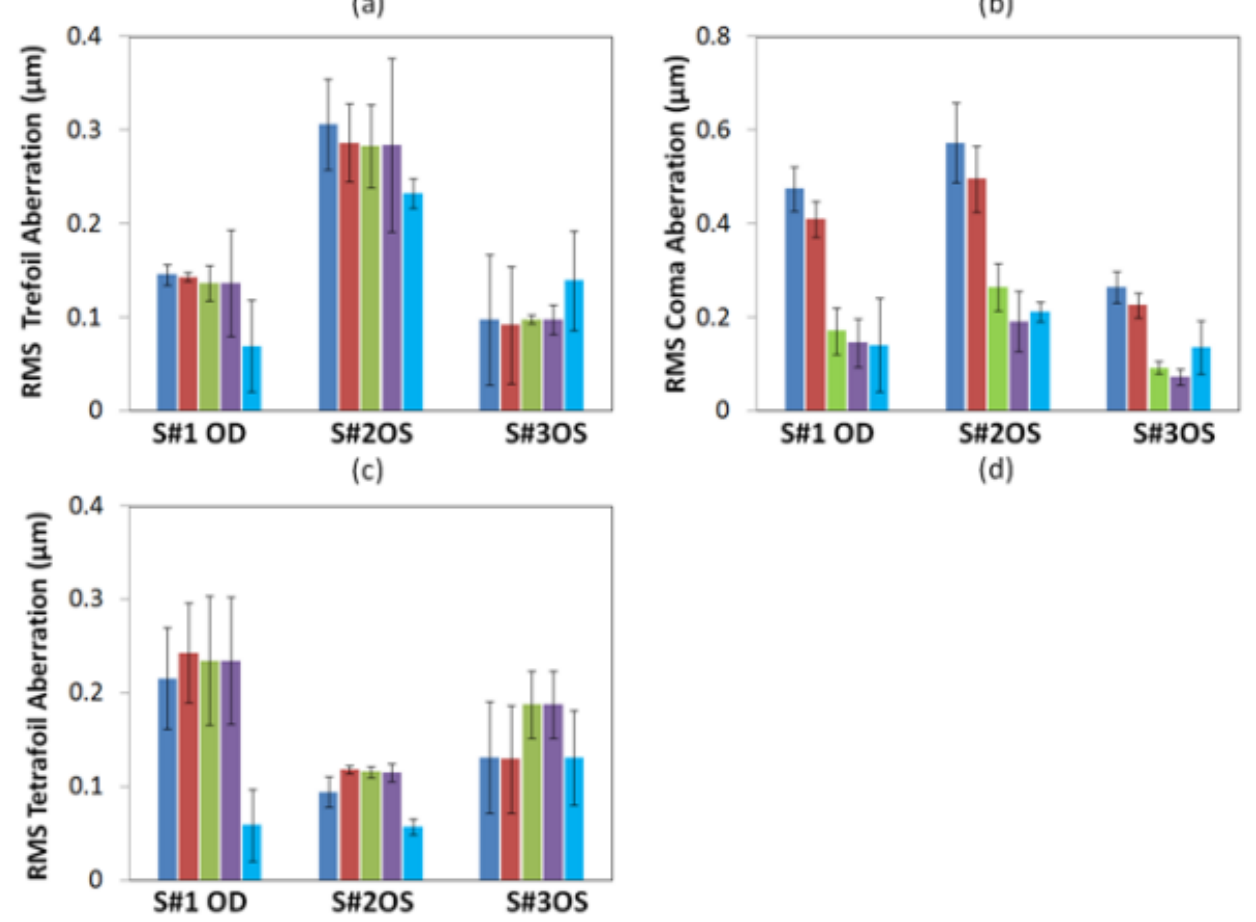

(e)

Figure 5.4 (a) RMS Astigmatism; (b) RMS Spherical Aberration; (c) RMS Trefoil; (d) RMS Coma; (e) RMS Tetrafoil, for anterior corneal RMS (blue bars), total corneal RMS (red bars), simulated total eye RMS assuming centered IOL (green bars), simulated total eye RMS assuming real IOL tilt/decentration (purple bars); and experimental (LRT) total eye aberration (light blue bars).

Figure 5.5 shows the linear correlations between total measured and simulated Zernike terms (astigmatism and high order aberrations) for the three eyes. There is a highly statistically significant correlation between the LRT-measured Zernike coefficients and those simulated from computer eyes model that incorporate all measured geometrical parameters (average slope $=0.82 \pm 0.12$; average coefficient of correlation $\mathrm{R}=0.93 \pm 0.05$; $\mathrm{p}<0.0001)$. The correlations, although statistically significant, are lower between the LRTmeasured Zernike and the anterior corneal Zernike terms (average slope $=0.69 \pm 0.21$; 
average coefficient of correlation $\mathrm{R}=0.73 \pm 0.05 ; \mathrm{p}<0.0001$ ); the LRT-measured Zernike and the entire (anterior+posterior) corneal Zernike terms (average slope $=0.92 \pm 0.23$; average coefficient of correlation $\mathrm{R}=0.88 \pm 0.03$; $\mathrm{p}<0.0001$ ); and similar to the correlations between LRT-measured Zernike terms and simulated total aberrations from computer eye models assuming centered IOLs (average slope $=0.87 \pm 0.1$; averagecoefficient of correlation $\mathrm{R}=0.94 \pm 0.04 ; \mathrm{p}<0.0001$ ). Incorporating posterior cornea and IOLs clearly improves the correlation, however including IOL tilt and decentration has only a minor impact as also reported by Rosales and Marcos (Rosales and Marcos 2007).
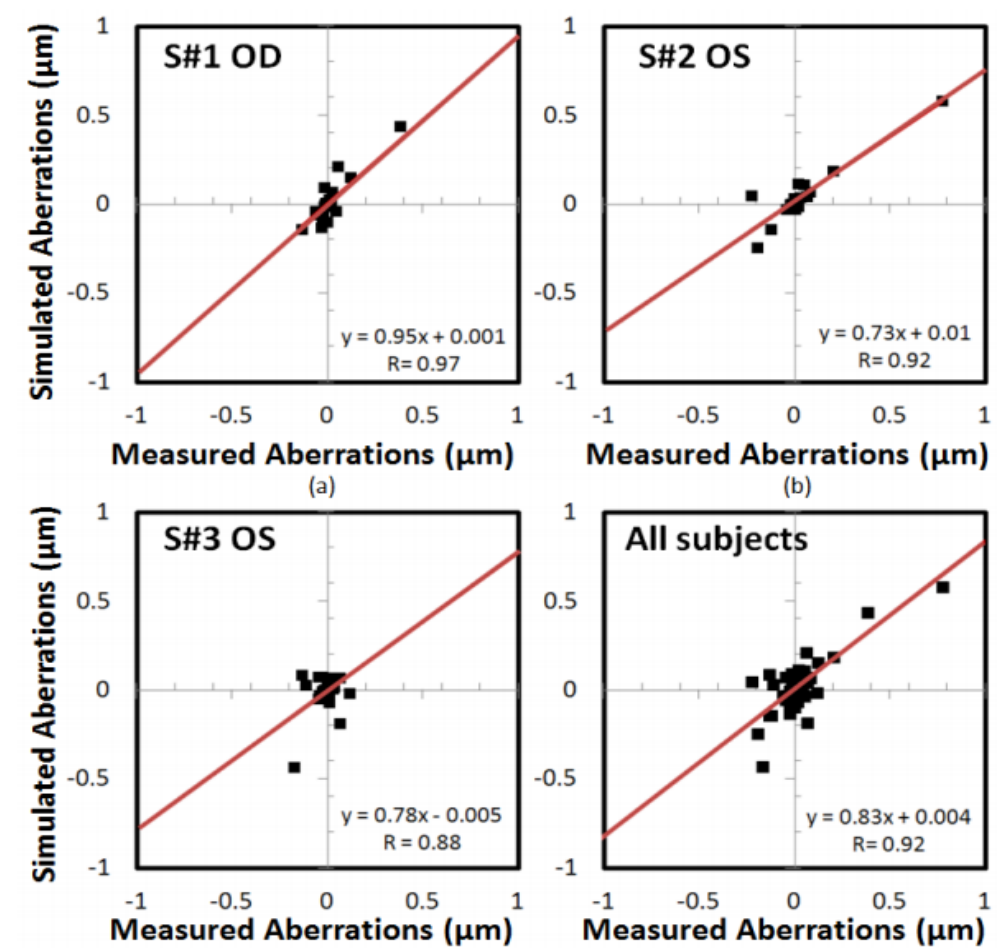

(c)

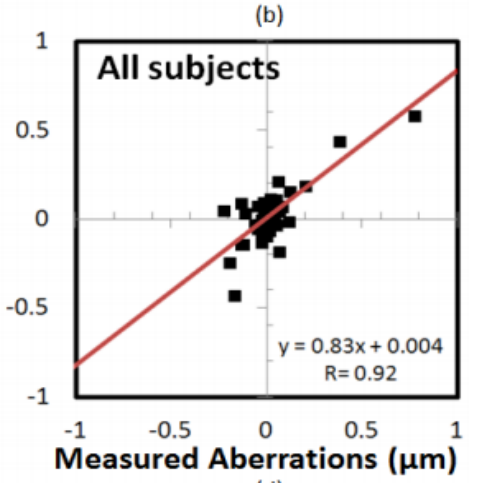

(d)

Figure 5.5 Linear correlations between measured and simulated Zernike coefficients (astigmatism and 3rd and higher orders) in (a) S\#1 OD; (b) S\#2 OS; (c) S\#3 OS; (d) combining data from the three subjects (72 points). Data are for 5-mm pupil diameters.

\subsubsection{Corneal/IOL compensation}

Comparison of the corneal aberrations to total aberrations (Figure 5.4) allows evaluating compensatory effects between optical elements for different terms. We did not find a compensation of anterior corneal astigmatism by the posterior corneal astigmatism: in fact, total corneal astigmatism was higher than anterior corneal astigmatism in all three eyes (by $24.56 \%$ on average). In contrast, the posterior cornea consistently partially compensated anterior corneal spherical aberration (by $25.71 \%$, on average) and coma (by $15.88 \%$, on average). Consistent with aspheric IOL designs (Marcos, Barbero et al. 2005, Marcos, Rosales et al. 2008), the IOLs compensated spherical aberration (by $72.14 \%$, according to the simulations; and by $77.06 \%$, by comparison of corneal and experimentally measured total aberrations) and coma (by $63.88 \% / 56.97 \%$, on average). 


\subsubsection{Effect of IOL tilt and decentration on optical quality}

The impact of the measured IOL tilt and decentration on optical aberrations appears to be minor, as revealed by the small differences in the simulated aberrations with the IOL aligned with the pupillary axis and those incorporating the real IOL tilt and decentration $(0.04 \mu \mathrm{m}$ for astigmatism and $0.04 \mu \mathrm{m}$ for coma, on average in Figure 5.4 (a) and (d)). In fact, the presence of IOL tilt and decentration resulted in a slight decrease in the RMS in all three eyes (by $10.84 \%$ in RMS for astigmatism and higher order terms, on average). To further compare simulations and predictions, and analyze the effect of IOL tilt and decentration on optical quality, we computed the corresponding MTFs (radial profiles) for astigmatism and higher order terms (Figure 5.6 (a)) and $3^{\text {rd }}$ and higher order aberrations (Figure 5.6 (b)) from wave aberrations simulated with centered IOLs, as well as IOL with real tilt/decentration and from LRT-measurement.
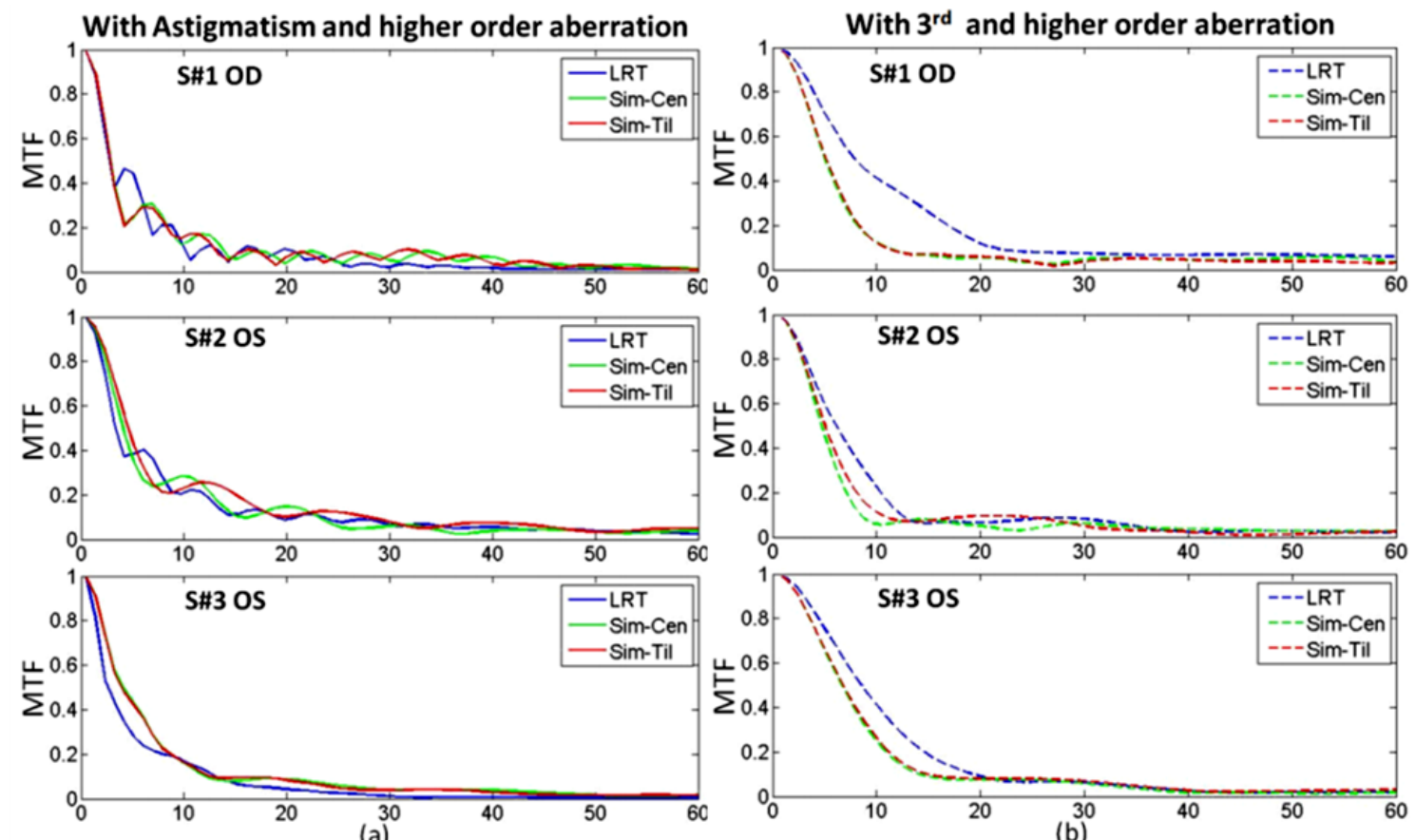

(a)

(b)

Figure 5.6 (a) MTFs (radial profiles) for astigmatism and higher order terms (b) MTFs (radial profiles) for 3rd and higher order aberrations, for LRT (blue lines), IOL with no tilt/decentration (green lines) and IOL with real tilt/decentration (purple lines). Data are for the three subjects, for 5-mm pupils.

The presence of IOL tilt/decentration improved optical quality (Visual Strehl) by $8.21 \%$ on average (Figure 5.7). 


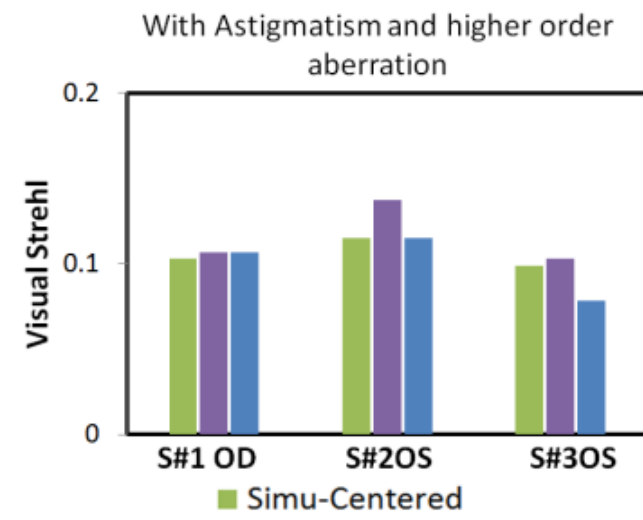

(a)
With $3^{\text {rd }}$ and higher order aberration

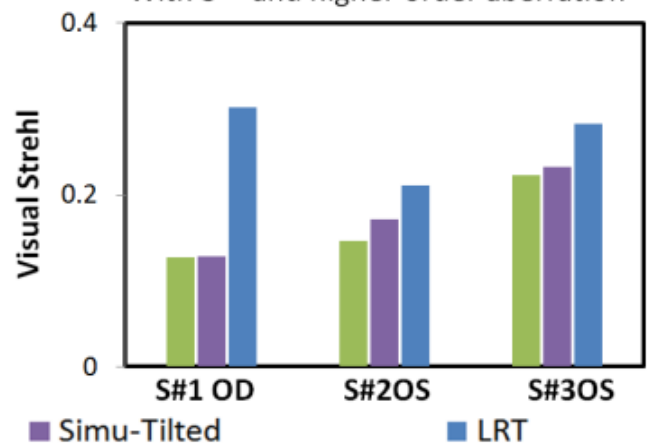

(b)

Figure 5.7 (a) Corresponding Visual Strehl with Astigmatism and $3^{\text {rd }}$ and higher order aberration (b) Corresponding Visual Strehl with $3^{\text {rd }}$ and higher order aberration without Astigmatism (green bars present Simulation with IOL centered, purple bars present simulation with IOL tilt and decentration, blue bars present LRT). 


\subsection{Discussion}

We have presented a customized computer eye model, using individual geometrical data in eyes with intraocular lenses obtained from quantitative 3D anterior segment OCT. The model predicted the wave aberrations measured in the same eyes with LRT aberrometry within $0.12 \mu \mathrm{m}$ of accuracy (RMS for astigmatism and high order aberrations, for 5-mm pupils).

The geometrical quantification capabilities of the OCT and its accuracy, presented in previous works (Ortiz, Perez-Merino et al. 2013), allows exact ray tracing on computer eye models using this geometrical information, provided that the refractive indices of the cornea and intraocular media are known. Pseudophakic eyes are simpler than phakic eye models as the refractive index of the IOL material is known from the specifications of the manufacturer. Knowledge of the Abbe number of the IOL material would allow extrapolating the index of refraction from the visible to other wavelengths (i.e., IR typically used in wavefront sensing). Nevertheless, chromatic effects are primarily of impact on defocus, and not high order aberrations (Perez-Merino, Dorronsoro et al. 2013, Vinas, Dorronsoro et al. 2015), which explains the good correspondence between LRT and simulated values, even if measurements were done in IR and simulations in green (i.e., the wavelength for which the indices of refraction of the IOL materials were available). In contrast, in phakic eyes the crystalline lens exhibits a patient/age-dependent Gradient Index Distribution (de Castro, Birkenfeld et al. 2013, Birkenfeld, de Castro et al. 2014). Computer eye models had been presented before (Tabernero, Piers et al. 2006, Rosales and Marcos 2007), using biometric information gathered from multiple devices (anterior cornea from Placido disk topography; tilt and decentration from Purkinje imaging apparatus; anterior chamber depth from slit lamp; axial length from low coherence interferometer). We found an average coefficient of correlation between measured and simulated aberration to be $\mathrm{R}=$ 0.93 . This compares with average coefficient of correlation $\mathrm{R}=0.82$ in Rosales and Marcos (Rosales and Marcos 2007) and average coefficient of correlation $\mathrm{R}=0.88$ in Tabernero et al. reports (Tabernero, Piers et al. 2006).

This chapter shows the capability of building computer eye models from information all obtained simultaneously with the same OCT system. Although our system did not have sufficient range to measure the axial length of the eye (and this information was obtained using low coherence interferometer on another device), different methods have been proposed to extend the range of the OCT to allow for axial length measurements. These methods include a dual OCT system rapidly flipping between anterior and posterior segment imaging and MEM-based swept source OCT (Grulkowski, Liu et al. 2012, Ruggeri, Uhlhorn et al. 2012).

The measurement of ocular aberrations in pseudophakic eyes allows assessment of the overall optical performance (Barbero, Marcos et al. 2003). Customized eye models in combination with the actual measurements of ocular aberrations can be used to identify the relative importance of each factor. Our results in eyes implanted with aspheric IOLs show the compensation of part of the positive spherical aberration of the cornea with the IOL (by $66.43-85.89 \%$ ). This is in agreement with the compensation reported with aspheric IOLs from different manufacturers (i.e. the Acrysof IQ (Alcon) by 66\%) (Marcos, Rosales et al. 
2008). Very interestingly, as shown by Marcos et al. (Marcos, Rosales et al. 2008), aspheric IOLs also do appear to compensate for coma, particularly horizontal coma. We found a coma compensation of 59.43\% with Asphina, and Marcos et al. (Marcos, Rosales et al. 2008) a compensation of $87 \%$ with Acrysof and Tecnis Aspheric IOLs. This compensation arises by a passive mechanism, from the combination of positive spherical aberration in the cornea and negative internally, and eye rotation, producing coma of opposite signs in the cornea and lens. Also in agreement with previous conclusions based on customized computer eye models (Rosales and Marcos 2007), IOL tilt and decentration appear to play a minor role in the optical quality of the eye.

Previous customized computer eye models revealed a major role of the cornea in the ocular aberrations of the pseudophakic eyes, which also occurs in the three eyes of our study. However, generally those computer models did not incorporate real posterior corneal elevations (i.e. Rosales et al. (Rosales and Marcos 2007) used a typical spherical posterior surface, Tabernero et al. (Tabernero, Piers et al. 2006)used a one-surfaced cornea). Several authors found a compensatory role of the anterior corneal astigmatism by the posterior cornea $[14 \%$ in Dunne et al. (Dunne, Royston et al. 1991) and 31\% in Dubbelman (Dubbelman, Sicam et al. 2006)]. Age-dependent changes of the anterior corneal astigmatism (i.e. astigmatism changes from with-the-rule to against-the-rule with age) may alter this balance. We did not find a lower total corneal astigmatism than that of the anterior cornea alone, suggesting no compensation in the three eyes of the study. Interestingly, the astigmatic axes of the anterior and posterior cornea do not appear aligned in all cases (Figure 5.3, relative angles ranging from 2.3 to 56.7 degree). Axis misalignment of the anterior and posterior corneal astigmatism has been reported before (Kamiya, Shimizu et al. 2015) as well as misalignments in the astigmatic axes of the anterior and posterior crystalline lens surfaces (Birkenfeld, de Castro et al. 2013, Sun, Birkenfeld et al. 2014). We did find consistent compensation by the posterior cornea of the anterior corneal spherical aberration and of anterior coma. The large differences in trefoil and tetrafoil might be explained by the larger fitting area of corneal surfaces in the 6-mm area while LRT was obtained for 5-mm pupil diameters.

The capability of OCT-based computer eye customized models to predict the measured aberrations post-operatively with a given implanted IOL opens the possibility to use those models as platforms to evaluate pre-operatively potential results with other IOLs, to optimize the selection of the IOL and to improve optical outcomes. Given the changes induced by small-incision cataract surgery on corneal aberrations, the model could be further refined by incorporating those induced aberrations to the pre-operative cornea to better reflect the post-operative corneal elevation maps. Marcos et al. (Marcos, Rosales et al. 2007) reported consistent significant changes in vertical astigmatism, trefoil and tetrafoil in patients in post-operative corneas of patients with superior 3.2-mm incisions. Neglecting intersubject and/or meridional variability in the corneal biomechanical response to the incision, these values could be incorporated on the appropriate Zernike terms in the model. Adjustments may be needed for different incision sizes for the clinical relevance of smaller incision sizes and physiological pupil diameters in probably minor.

OCT-based custom computer eye models are also applicable to the optimization of the IOL power calculation, using virtual ray tracing on these eyes. Ray tracing IOL power 
calculation overcomes many limitations of current IOL power selection techniques. These are typically based on regression models obtained for a large patient population, as opposed to the individualized approach based on customized eye models. Pre-operative measurements of anterior and posterior corneal surfaces overcome the limitations of the standard IOL power calculations which only make use of anterior corneal keratometry and the so-called keratometric index. Future analysis of the pre-operative anterior segment anatomy and crystalline lens from 3D OCT quantitative imaging will allow better predictions of the estimated lens position. Similar to the comparisons between the measured and simulated high order aberrations addressed in the current study, validations of the IOL power selections will involve comparisons of measured and predicted aberrometric defocus and/or refraction.

In summary, OCT-based customized computer eye models are excellent predictive tools of the optical quality in pseudophakic eyes, allow understanding the factors that contribute to optical degradation and hold promise to become a primary tool to optimize the selection of the IOL to be implanted in a cataract procedure.

In the following chapters we will address in particular the contribution of IOL tilt and decentration to optical quality in pseudophakic eyes and the selection of the optimal IOL, based on the OCT-based computer model eyes developed in this chapter. 


\section{CHAPTER 6}

\section{OCT-BASED RAY TRACING}

ON PSEUDOPHAKIC EYES TO

IDENTIFY OPTIMAL IOL CENTRATION 
The contribution of Mengchan Sun to the study, in collaboration with other coauthors, was the literature search, the design of the experiments and the data acquisition (with Pablo Pérez-Merino), the development of specific routines and processing of the data (with Edurado Martinez-Enriquez), and the simulation design simulation and data analysis. This work was also presented at the 2015 Annual Meeting of the Association for Research in Vision and Ophthalmology (ARVO) in Denver (Colorado, USA). 


\section{RESUMEN}

Objetivos: La Tomografía de Coherencia Óptica Cuantitativa en 3D (OCT) proporciona información anatómica completa para construir modelos de ojo personalizados para identificar la posición lateral de la IOL que produciría la mejor calidad óptica.

Métodos: Se midieron 3 sujetos con IOL asféricas monofocales (Bausch y Lomb, US, Alcon, US) 3 meses después de la cirugía de cataratas. Se midió la geometría del segmento anterior cuantitativo en 3D (topografía corneal, profundidad de la cámara anterior, inclinación y descentramiento de la IOL, posición de la fóvea) usando un OCT espectral y las aberraciones mediante trazado de rayos láser, ambos de desarrollo propio y descritos en el capítulo anterior. Las aberraciones oculares se estimaron mediante trazado de rayos virtual en el programa ZEMAX, en un modelo de ojo personalizado al ojo del paciente. Se calcularon las aberraciones de onda, y la calidad óptica correspondiente en términos de Visual Strehl (VS), para pupilas de $3 \mathrm{~mm}$, considerando el descentramiento real, y para descentramientos horizontales y verticales de $-1 \mathrm{a}+1 \mathrm{~mm}$ en pasos de $0.2 \mathrm{~mm}$, con respecto del centro de la pupila. El descentramiento óptimo se estima como aquél que maximiza VS.

Resultados: El descentramiento lateral de la IOL produce variaciones en la VS. Las diferencias medias entre el VS máximo y real (incluyendo astigmatismo) fueron 0.06, 0.01 y 0.08 (calidad óptica mejorada de 7.29\%, 2.83\% y 9.83\%) de E\#1 OD, E\#2 OS y E\#3 OS respectivamente. Y $0.01,0.02$ y 0.05 (calidad óptica mejorada en $1.05 \%, 3.23 \%$ y $5.35 \%$ ) de E\#1 OD, E\#2 OS y E\#3 OS, respectivamente, para aberraciones de alto orden solo. Las posiciones óptimas de IOL (VS máximo incluyendo astigmatismo y HOA) en E\#1 OD, E\#2 OS y E\#3 OS son $(0.20,0.40),(-0.20,-0.60)$ y $(-0.80,0.80) \mathrm{mm}$, y $(0.20,0.20),(-0.20,-$ $1.00)$ y $(-0.60,1.00) \mathrm{mm}$, respectivamente, para aberraciones de alto orden solo. La diferencia entre la posición óptima de IOL) y posición IOL real de E\#1 OD, E\#2 OS y E\#3 OS son $0.21,0.81,0.85 \mathrm{~mm}$ respectivamente en el eje horizontal y $0.28,0.41$ y $0.48 \mathrm{~mm}$ en el eje vertical respectivamente (incluyendo astigmatismo) y son $0.21,0.81$ y $0.65 \mathrm{~mm}$ en el eje horizontal respectivamente y $0.08,0.81$ y $0.68 \mathrm{~mm}$ en el eje vertical, respectivamente (para aberraciones de alto orden)

Conclusiones: Los modelos oculares personalizados basados en OCT predicen la calidad óptica de los ojos pseudofáquicos implantados con lentes intraoculares monofocales de última generación. En ojos reales, una IOL totalmente alineada con el centro de la pupila no produce la mejor calidad óptica. El descentramiento óptimo de la IOL puede obtenerse para cada paciente basándose en modelos de ojo personalizados, y la mejora conseguida puede ser de relevancia clínica. 
OCT-based ray tracing on pseudophakic eyes to identify optimal IOL centration

\subsection{Introduction}

More than 6,000 cataract procedures/million people are performed yearly in developed countries, and cataract surgery has benefited significantly from technological advances. Advanced intraocular lens (IOL) designs aim at providing the best possible optical quality, in accordance with the patient's need (Atchison 1989, Atchison 1991, Holladay, Piers et al. 2002). As lenses become increasingly complex, knowledge of geometry and intraocular positioning will become even more important (Wolffsohn and Davies 2007). Current cataract surgeries most frequently involve implantation of posterior chamber IOLs within the capsular bag; an approach, according to some authors, that makes it difficult to avoid decentration (Taketani, Matuura et al. 2004). Studies using Purkinje imaging have observed decentrations of the lens in the horizontal direction, generally with temporal decentrations in left eyes and nasal decentrations in right eyes (Rosales and Marcos 2006). Vertical decentrations (generally lower in magnitude) have also been reported in some patients (de Castro, Rosales et al. 2007). The magnitude of decentrations varies across studies, likely in part due to the different techniques used to asses lens decentrations, and the axis of reference to which those are referred, with most reports ranging from 0.3 to $0.7 \mathrm{~mm}$ (Phillips, Perez-Emmanuelli et al. 1988, Ohara, Kato et al. 2006, Rosales, De Castro et al. 2010, Crnej, Hirnschall et al. 2011, Holladay, Calogero et al. 2017). While in some clinical decentration is estimated from direct view of the lens through the cornea (Chang and Waring 2014), several studies present technologies to measure IOL tilt and decentration taking into account the refraction of the cornea and the non-centered nature of the optical system of the eye. Dedicated technology for lens tilt and decentration measurements include Purkinje imaging (Phillips, Perez-Emmanuelli et al. 1988, Guyton, Uozato et al. 1990, Nishi, Hirnschall et al. 2010), distortion-corrected Scheimpflug imaging (Rosales, De Castro et al. 2010), quantitative anterior segment Optical Coherence Tomography (Ortiz, Siedlecki et al. 2009, Marcos, Ortiz et al. 2014) and en-face OCT (Sun, de Castro et al. 2014). Ray tracing analysis on computer eye models based on individual anatomical information that includes IOL tilt and decentration allow evaluating the impact of IOL alignment on the wave aberrations of the eye, and pontential impact on ocular astigmatism an coma (Li, Wang et al. 2013) (Baumeister, Buhren et al. 2009).

Lens decentration may be associated with a failure to place the lens correctly in the capsular bag during surgery (called subluxation), or it may develop in the postoperative period because of external (e.g., trauma, eye rubbing) or internal forces (e.g., scarring, peripheral anterior synechiae (PAS), capsular contraction, size disparity), more notably fibrotic processes and capsular bag shrinkage (Tappin and Larkin 2000). This event may be the result of a combination of a small capsular bag and a mechanically unstable haptic platform or lens material (Kohnen 1998). In any case, in state-of-the-art lens platforms, the data suggest that the lens decentrations are primarily driven by the decentrations of the natural lens capsule in the eye, and that this is mostly preserved after surgery (Rosales and Marcos 2006, Rosales, Wendt et al. 2008, Rosales, De Castro et al. 2010, Marcos, Ortiz et al. 2014). A notable exception was observed in patients implanted with the accommodating IOL Crystalens, very likely associated with the hinge nature of the haptics and asymmetric fibrosis in the haptic region (Zsyndrome in Crystalens) (Marcos, Ortiz et al. 2014). Furthermore, Rosales and Marcos using custom model eyes (built by gathering biometric information from multiple instruments, included IOL decentrations from multiple 
instruments) found that in a large proportion of patients $(58.3 \%$ on 12 eyes of 7 pseudophakic patients) the actual IOL decentrations in fact improved optical quality with respect to that of an IOL with no decentrations, suggesting favorable interactions between lens decentrations and the off-axis position of the fovea (Rosales and Marcos 2007).

A remaining question is if this anatomical decentration is actually optimal or whether optical quality could be further improved by an optimal position of the lens. Capsular tension rings (CTRs) have been shown to play a role in the stability and positioning of IOLs (Lee, Lee et al. 2001, Alio, Elkady et al. 2008), very likely preserving the natural decentration of the capsular bag. However, it is technically conceivable to fine tune the decentration of the IOL, if an optimal lateral positioning resulted in a visual benefit. For example, a published patent reports the design of an IOL with asymmetric haptics, which allowed the centration of the IOL optic with respect to the pupil in order to reduce dsyphotopsia and/or the perception of dark shadows (Michael J. Simpson 2007). An accurate orientation of the haptic along the meridian at which decentration is aimed could be achieved through standard methods in implantation of rotational asymmetric multifocal Intraocular lens (MIOL) (Venter, Pelouskova et al. 2013, Kretz, Khoramnia et al. 2016) or toric lenses. Some commercial surgical microscopes, e.g. IOLcompass Pro (Leica Microsystem, Wetzlar, Germany) and VERION $^{\mathrm{TM}}$ Digital Marker (Alcon, Fort Worth, Texas, United States), support ophthalmic surgeons in providing stable and accurate guidance for intra-operative positioning of premium intraocular lenses (IOLs) throughout the cataract surgical workflow.

In Chapter 3, we have presented an OCT-based method to measure tilt and decentration of the lens in vivo. On the other hand, in Chapter 5, we have presented full OCT-based custom eye models built using anatomical information from patients, which are able to predict with great accuracy the measured aberrations of the eye, with $>90 \%$ correspondence. In this study we used these OCT-based custom eye models to investigate the changes in optical quality in patients implanted with IOLs with IOL position, and in particular searched for the lens position that optimized optical quality at the patient's level. 
OCT-based ray tracing on pseudophakic eyes to identify optimal IOL centration

\subsection{Methods}

\subsubsection{Patients, surgery and measurements}

Three eyes from two patients (64 and 78 years old) implanted with three types of IOLs (Akreos AO M160, 20D, Bausch and Lomb, Rochester, New York, US; Acrysof IQ SN60WF, 21.5D, Alcon, Texas, US; CT Asphina 409M, 20.5D, Zeiss, Jena, Germany) were investigated. E\#1 and E\#3 are right and left eyes from subject 1. Standard phacoemulsification intra-capsular cataract surgery had been performed by a single surgeon at Fundación Jiménez Díaz Hospital (FJD), with 2-mm corneal incisions (temporal in OD and nasal in OS). Total wave aberrations were measured using a custom-developed Laser Ray Tracing (LRT) system (Llorente, Barbero et al. 2004). Anterior segment geometry (cornea, iris and IOL) was measured using a custom-developed spectral OCT (Ortiz, Siedlecki et al. 2009, Ortiz, Siedlecki et al. 2011, Ortiz, Perez-Merino et al. 2013). Measurements were done under mydriasis $(2.5 \%$ phenylephrine).

\subsubsection{Laboratory setup: OCT, LRT and Experimental protocols}

The sOCT system used in the measurements has been described in detail in Section 2.1.1. Measurements were collected in a $10 \times 10 \mathrm{~mm}$ area, and consisted of a collection of $50 \mathrm{~B}$ scans composed by 300 A-scans. Sets of 3D images of the anterior segment were captured along the pupillary axis approximately 5 seconds after blinking. The off-axis position of the fovea (angle lambda, $\lambda$ ) is calculated from the eye rotation between alignments of both optical axis and pupillary axis. The automatic image processing tools for denoising, segmentation, clustering, merging, and biometric and IOL alignment measurements have been described in detail before in Section 2.6.2 (MATLAB routines). Anterior and posterior corneal elevation maps, corneal thickness and anterior chamber depth were obtained from 3D OCT as described in Section 2.1.2 (Quantification tools).

Total wave aberrations, measured using custom-developed LRT at $785 \mathrm{~nm}$, were sampled at 37 positions of a $3 \mathrm{~mm}$ pupil under foveal fixation with a maximum energy exposure of $6.8 \mu \mathrm{W}$, which has been described in detail in Section 2.4.2. Measurements were done under mydriasis ( 1 drop 1\% tropic amide) at three weeks after cataract surgery. Prior to the measurement of total aberration, the patient adjusted his/her subjective refraction using a Badal optometer in the LRT system while viewing a stimulus on a minidisplay on the retinal conjugate plane.

\subsubsection{Purkinje-Like OCT Method}

IOL tilt and decentration was obtained by the Purkinje-like OCT method described in Section 2.1.3. In brief, the Purkinje-like analysis can be summarized in five steps (Figure 2.7): (1) collection of a volumetric data set; (2) generation of image intensity values in the axial direction; (3) pupil segmentation and identification of the positions of the specular reflexes on the different surfaces (Purkinje-like images positions, PI, PIII and PIV). The reflexes PI, PIII, and PIV are easily identified by checking the corresponding OCT B-scan image. Eye rotation $(\beta)$, lens tilt $(\alpha)$ and decentration $(d)$ were obtained from the inversion in Eq.2.4, using the positions of the experimental reflexes (PI, PIII and PIV). 
The tilt and decentration nomenclature and sign conventions are illustrated in Section 2.1.3, which is the same as Rosales et al. 2008 (Rosales and Marcos 2006). As a brief summary, positive horizontal decentration stands for nasal decentration (in ODs) and temporal decentration (in OSs), and vice versa for negative; positive vertical decentration for superior, and negative for inferior. Positive tilt/rotation around the horizontal axis (tilt/rotation $\mathrm{x}$ ) indicates that the superior edge of the lens is moved forward. Negative tilt/rotation around the vertical axis (tilt/rotation y) (in ODs) indicates that the temporal edge of the lens is moved forward, and (in OSs) indicates that the nasal edge of the lens is moved forward, shown in Figure $2.8(\mathrm{~g})$.

\subsubsection{Full OCT-based customized eye models}

Custom computer eye models were created for each patient, as described in Section 2.3.4. OCT-based elevation data from both anterior and posterior corneal surfaces, anterior segment distances, IOL geometry and alignment data, angle lambda, and axial length from the IOLMaster (Carl Zeiss AG, Jena, Germany) were exported to ZEMAX (Radiant ZEMAX; Focus software, Tucson, AZ, USA), where the custom model eyes were built. Refractive indices of cornea, aqueous humour, vitreous humour, Akreos IOLs and Asphina IOLs were $1.376,1.337,1.337,1.458$ and 1.460 respectively, at $555 \mathrm{~nm}$. Wave aberrations were calculated in the pupil plane by tracing an array of $64 \times 64$ collimated rays through the eye surfaces within a central $5-\mathrm{mm}$ pupil diameter area, at $555 \mathrm{~nm}$. Figure 2.15 illustrates the computation of aberrations from OCT data, i.e. ray tracing calculation on OCT distortion-corrected corneal and IOL surfaces. Data are analyzed in terms of Zernike terms and retinal image quality metrics using Visual Strehl (VS) (Iskander 2006). Wave aberrations were calculated by tracing an array of $64 \times 64$ collimated rays through the eye surfaces (anterior and posterior cornea, separated by corneal thickness, ACD, anterior and posterior IOL, separated by IOL thickness) in the $3 \mathrm{~mm}$ pupil plane, placed at $3.47 \mathrm{~mm}$ from the anterior corneal surface and retinal plane. Data are analyzed in terms of Zernike terms and retina image quality was calculated from measured/simulated wave aberrations in terms of Visual Strehl (VS), estimated for the native tilt $\&$ decentration.

\subsubsection{Estimation of optimal IOL decentration}

In order to identify optimal IOL centration, OCT-based ray tracing on a pseudophakic eye model was performed with all anatomical input data from the patient (anterior and posterior corneal elevation maps, IOL geometry, off-axis foveal angle, and IOL tilt), while decentration was changed from -1 to $+1 \mathrm{~mm}$ (in $0.2 \mathrm{~mm}$ steps) in a horizontal/vertical grid. In selected cases as a control, computer eyes were also created using simpler anterior and posterior corneal representations (aspheric surfaces), and setting tilt and/or eye rotation to zero. Coordinate break surfaces were introduced into the model, which allowed specifying the IOL location. Wave aberrations were calculated for every IOL position, assuming a 3$\mathrm{mm}$ pupil. Wave aberrations were fit with $6^{\text {th }}$ order Zernike polynomial expansions following OSA standards, and were described in terms of individual Zernike coefficients or RMS wavefront errors. Also, the PSF, MTF and OTF were computed using Fourier optics from the wave aberrations using routines written in MATLAB. Visual Strehl was calculated for the corresponding wave aberrations (for 3-mm pupils) for every IOL location. Also, VS was maximized through-focus (1.25D to $-1.25 \mathrm{D}$ in $0.125 \mathrm{D}$ steps), to 
calculate residual defocus errors. The lateral IOL position producing the maximum VS was taken as the optimal IOL position.

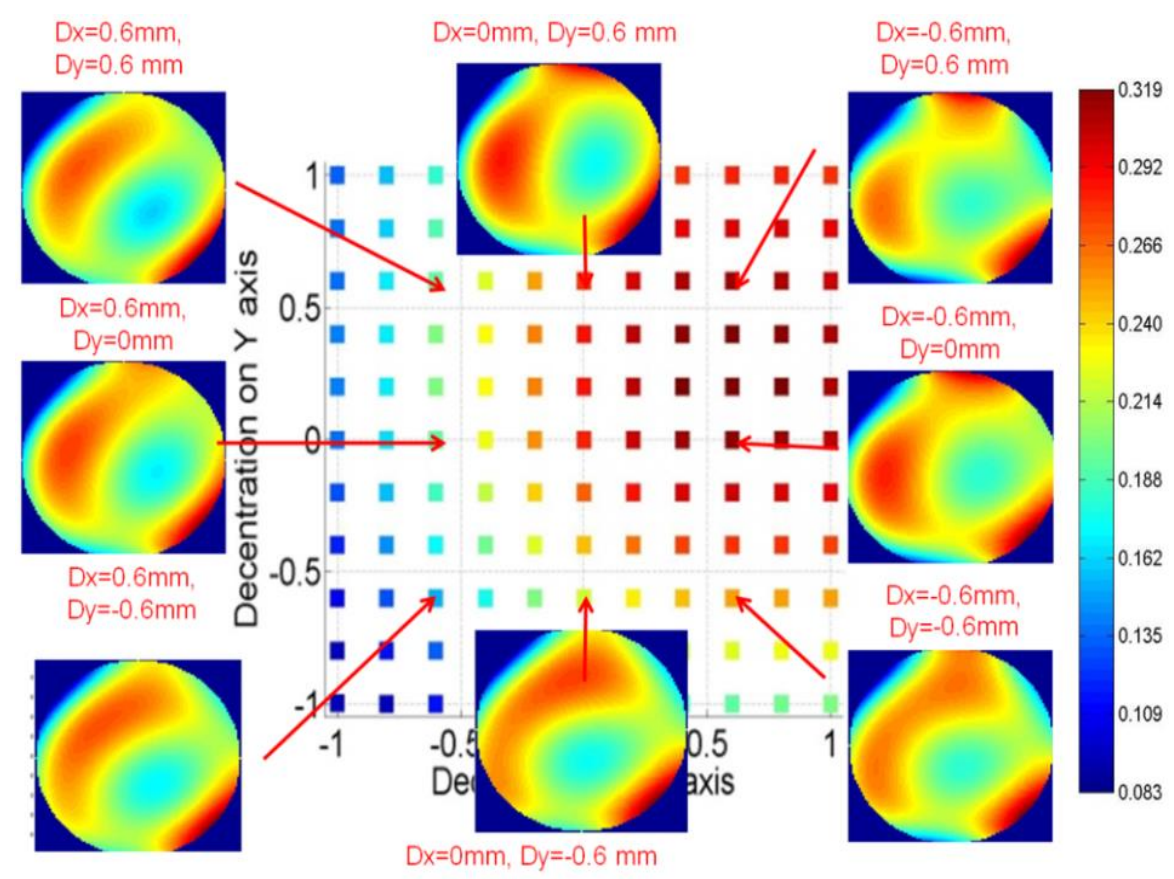

Figure 6.1 Illustration of estimation of the optical IOL position. (1) Custom model eye is created with individual anatomical information; (2) Wave aberrations are computed for different IOL decentrations $1 \mathrm{~mm} \times 1 \mathrm{~mm}$ grid (0.2 mm steps); 8 sample wave aberrations are shown; (3) Visual Strehl is calculated for every wave aberration (for 3-mm pupil diameter) and a VS pupillary map is created;(4) Location producing the maximum VS is identified. 


\subsection{Results}

\subsubsection{Measured eye rotation, IOL tilt and decentration}

IOL tilt, decentration and eye rotation were estimated as described in Chapter 2, and given in Table 6.1 for the 3 pseudophakic eyes of the study.

Table 6.1 show measurements of eye rotation, IOL tilt and decentration (horizontal and vertical components) for each eye.

\begin{tabular}{cccc} 
& E\#1 OD & E\#2 OS & E\#3 OS \\
\hline Eye rotation X (degree) & $1.33 \pm 0.46$ & $1.83 \pm 0.38$ & $0.14 \pm 0.27$ \\
\hline Eye rotation Y (degree) & $-4.12 \pm 0.21$ & $3.55 \pm 0.58$ & $2.43 \pm 0.78$ \\
\hline IOL tilt X (degree) & $2.22 \pm 0.49$ & $-1.04 \pm 0.41$ & $2.47 \pm 0.26$ \\
\hline IOL tilt Y (degree) & $0.26 \pm 0.39$ & $1.89 \pm 0.85$ & $1.21 \pm 0.35$ \\
\hline IOL decentration X (mm) & $-0.01 \pm 0.06$ & $0.61 \pm 0.05$ & $0.05 \pm 0.02$ \\
\hline IOL decentration Y (mm) & $0.12 \pm 0.19$ & $-0.19 \pm 0.16$ & $0.32 \pm 0.14$ \\
\hline
\end{tabular}

\subsubsection{Change of optical quality with IOL decentration using computer eye models with symmetric aspheric cornea}

VS pupillary maps were obtained using aspheric fits to the patient's anterior and posterior corneas. Four conditions were tested: (1) Assuming no eye rotation (ER) and no IOL tilt (IT); (2) Assuming the measured ER but no IT; (3) assuming no ER and the measured IT; (4) Assuming the measured ER and IT. Values of measured ER and IT are shown in Table 6.1. Figure 6.2 shows the Visual Strehl (VS) pupillary maps for one eye (E\#1 OD), including: astigmatism and high order aberrations (maximized VS thru-focus), only high order aberration (maximized VS thru-focus). For real ER and IT (last column), VS ranged from 0.51 to 0.69 across IOL decentrations for astigmatism and HOA; from 0.52 to 0.78 for HOA.

Even for symmetric aspheric corneas, interactions occur between tilt, decentration and eye rotation. In the absence of tilt and eye rotation, the optimal lateral position of the IOL corresponds to the pupil center, but the presence of other ocular misalignments shift substantially the best optimal IOL position. The large difference in the optimal lateral position predicted considering astigmatism and HOA, or only high order aberrations suggests important interactions between different aberration terms. 
OCT-based ray tracing on pseudophakic eyes to identify optimal IOL centration

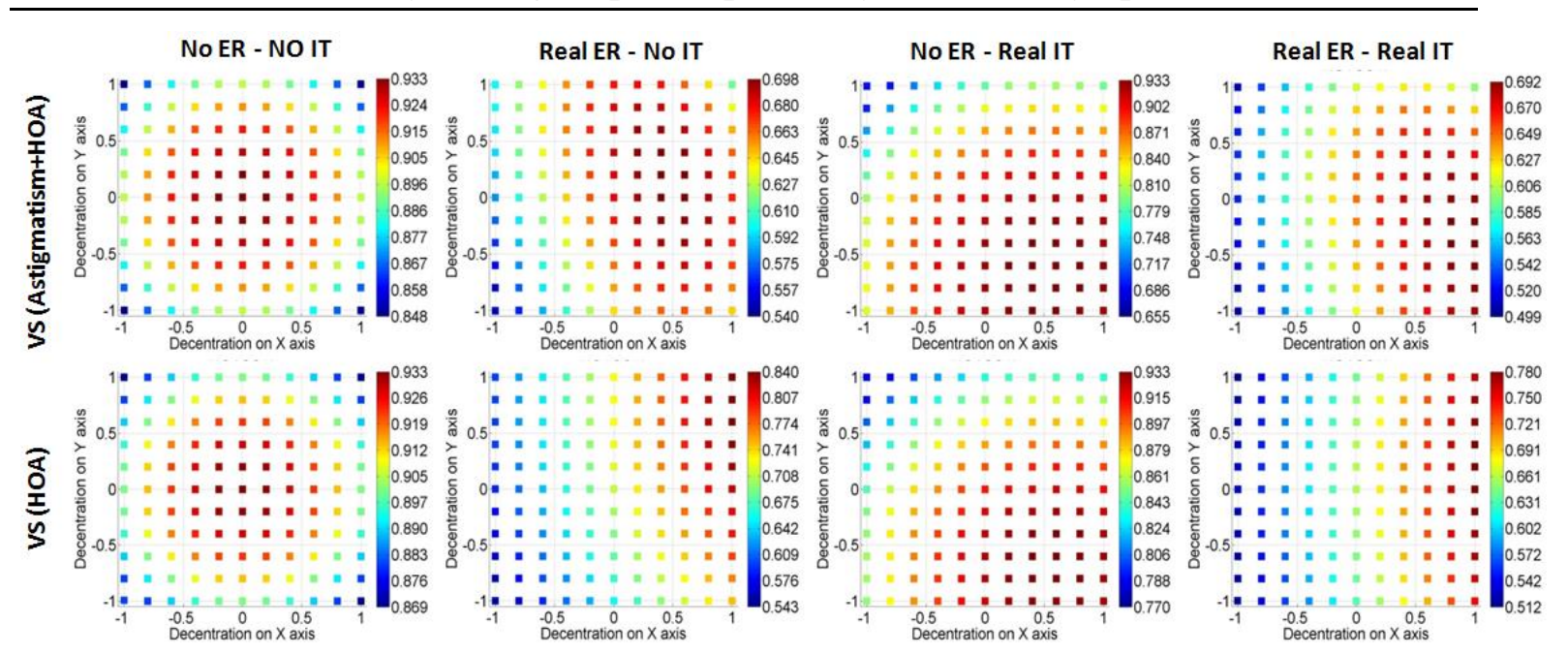

Figure 6.2 the Visual Strehl (VS) pupillary maps for one eye (E\#1 OD), including: astigmatism and high order aberrations (in the first row) and only high order aberration (in the second row) under four conditions (without eye rotation (ER) and IOL tilt (IT) in the first column; with ER and without IT in the second column; without ER but with IT in third column; with ER and IT in the fourth column)

\subsubsection{Change of optical quality with IOL decentration using computer eye models with real corneas}

Figure 6.3 shows similar maps as in Figure 6.2 for one eye (E\#1 OD) but now using anterior and posterior elevation maps (fitted to $6^{\text {th }}$ order polynomials). For real ER and IT (last column), VS ranged from 0.32 to 0.81 across IOL decentrations for astigmatism and HOA; from 0.68 to 0.86 for HOA.

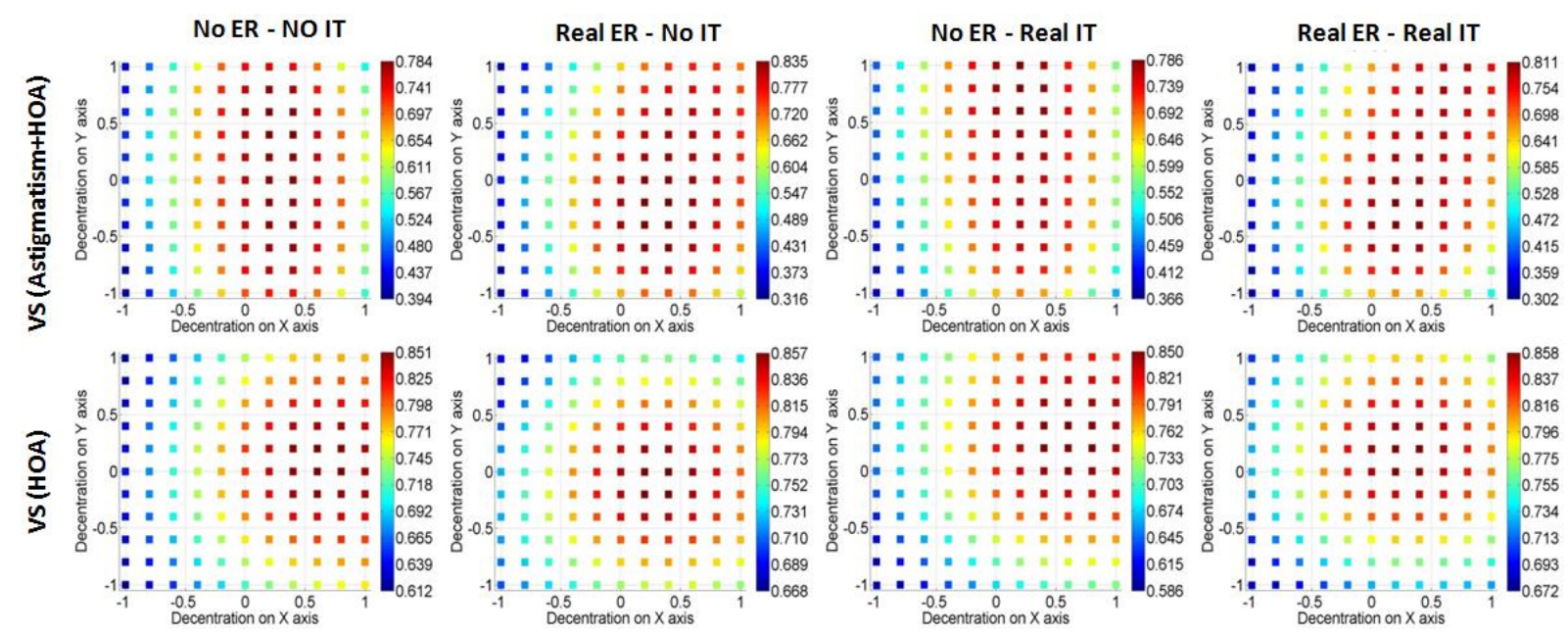

Figure 6.3 the Visual Strehl (VS) pupillary maps for one eye (E\#1 OD) using anterior and posterior elevation maps (fitted to $6^{\text {th }}$ order polynomials), including: astigmatism and high order aberrations (in the first row) and only high order aberration (in the second row) under the same conditions in Figure 6.2.

For real anterior and posterior corneal elevation maps, interactions also occur between tilt, decentration and eye rotation. In the absence of tilt and eye rotation, the optimal lateral position of the IOL does not correspond to the pupil center, and the presence of other ocular 
misalignments shift substantially the best optimal IOL position. The less difference in the optimal lateral position predicted considering only HOA, or also astigmatism aberrations suggest important interactions between different aberration terms compared to symmetric aspheric corneas. The comparison of maximum Visual Strehl and optimal IOL decentration between using symmetric and aspheric corneal and using real corneal elevations will be analysis in section 6.3.4.

Figure 6.4 shows Visual Strehl (VS) of E\#1 OD, E\#2 OS and E\#3 OS including astigmatism and high order aberrations (HOA) in the first row and including HOA only in the second row using anterior and posterior elevation maps (fitted to $6^{\text {th }}$ order polynomials), with real ER and real IT. The VS ranged from 0.41 to 0.50 (E\#2 OS) and 0.34 to 0.84 (E\#3 OS) across IOL decentrations for astigmatism and HOA; from 0.46 to 0.49 (E\#2 OS) and 0.54 to 0.84 (E\#3 OS) for HOA. The real VS included astigmatism and HOA, of E\#1 OD, E\#2 OS and E\#3 OS are 0.75, 0.49 and 0.75 respectively, with real IOL misalignment. The real VS included HOA only, of E\#1 OD, E\#2 OS and E\#3 OS are 0.85, 0.48 and 0.80 respectively, with real IOL misalignment.
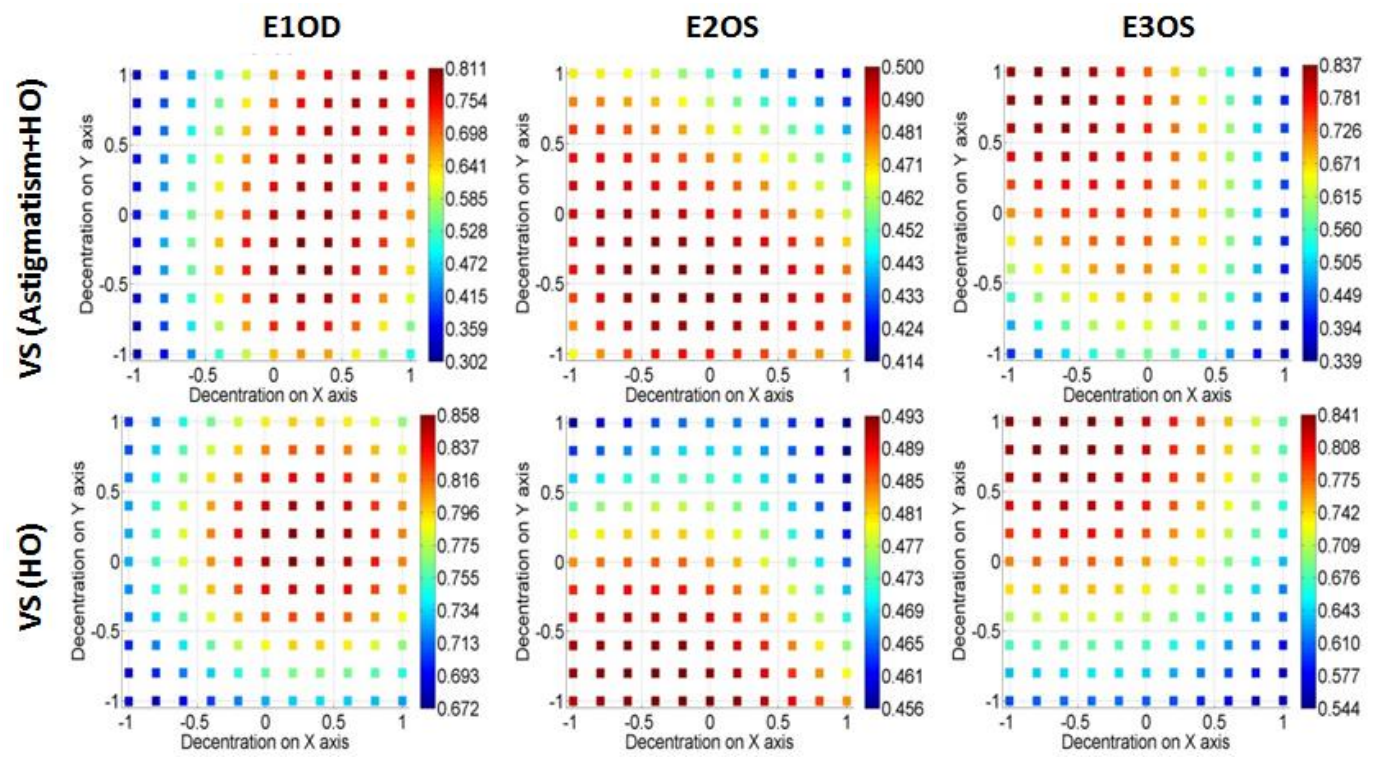

Figure 6.4 Visual Strehl (VS) of E\#1 OD, E\#2 OS and E\#3 OS including Astigmatism and high order aberrations (HO) in the first row and including HOA only in the second row, with real ER and real IT.

Across subjects, the optimal lateral position of the IOL does not correspond to the pupil center and the presence of other ocular misalignments shift nasally the best optimal IOL position. The less difference in the optimal lateral position predicted considering only high order aberration, or also astigmatism suggest important interactions between different aberration terms in the pair of eye (E\#1 OD and E\#3 OS) compared to E\#2 OS. The comparison of maximum Visual Strehl and optimal IOL decentration between using symmetric and aspheric corneal and using real corneal elevations will be analysis in section 6.3.4. 


\subsubsection{Maximum Visual Strehl and Optimal IOL decentration}

Figure 6.5 (a) compares the maximum VS achieved by optimally placing the IOL, assuming aspheric corneas (blue lines) or real corneal elevation maps (red lines), in comparison with a perfect centered IOL, for subject E\#1 (derived from the maps shown in Figure 6.2 and 6.3). In the absence of corneal irregularities, a very high VS (0.9) is achieved in the absence of ER and IT, or in the presence of IT alone, for a practically centered IOL. However, in presence of ER (or ER and IT), optimizing the position of the IOL can improve VS by $15.78 \%$, considering HOA only, but not significantly considering astigmatism and HOA. The presence of astigmatism and HOA in the cornea minimizes in general the impact of ER. Optimizing IOL decentration improves VS by around $4.85 \%$ in all conditions with respect to a perfectly centered IOL and by $7.29 \%$ (lines not shown in graph) with respect to the real IOL decentration.

Figure 6.5 (b) shows the IOL position maximizing VS (for aspheric corneas, in blue and for real surface elevation in red) for the different conditions considering astigmatism and HOA (closed symbols) or HOA only (open symbols), in comparison with the real IOL position (star symbol), for subject E\#1. While in all cases the optimal IOL position is decentered nasally, there is a large scatter across conditions. For the eye model most closely representing the patient's eye (real surface elevation; real IOL tilt; real eye rotation) the coordinates of the IOL position optimizing retinal image quality (for astigmatism and HOA) are $0.4 \mathrm{~mm}$ inferiorly and $0.2 \mathrm{~mm}$ nasally. The presence of high order irregularities in the cornea results in large changes in the optimal position of the IOL, indicating that both ocular surface misalignments and surface irregularities contribute to optical degradation. 

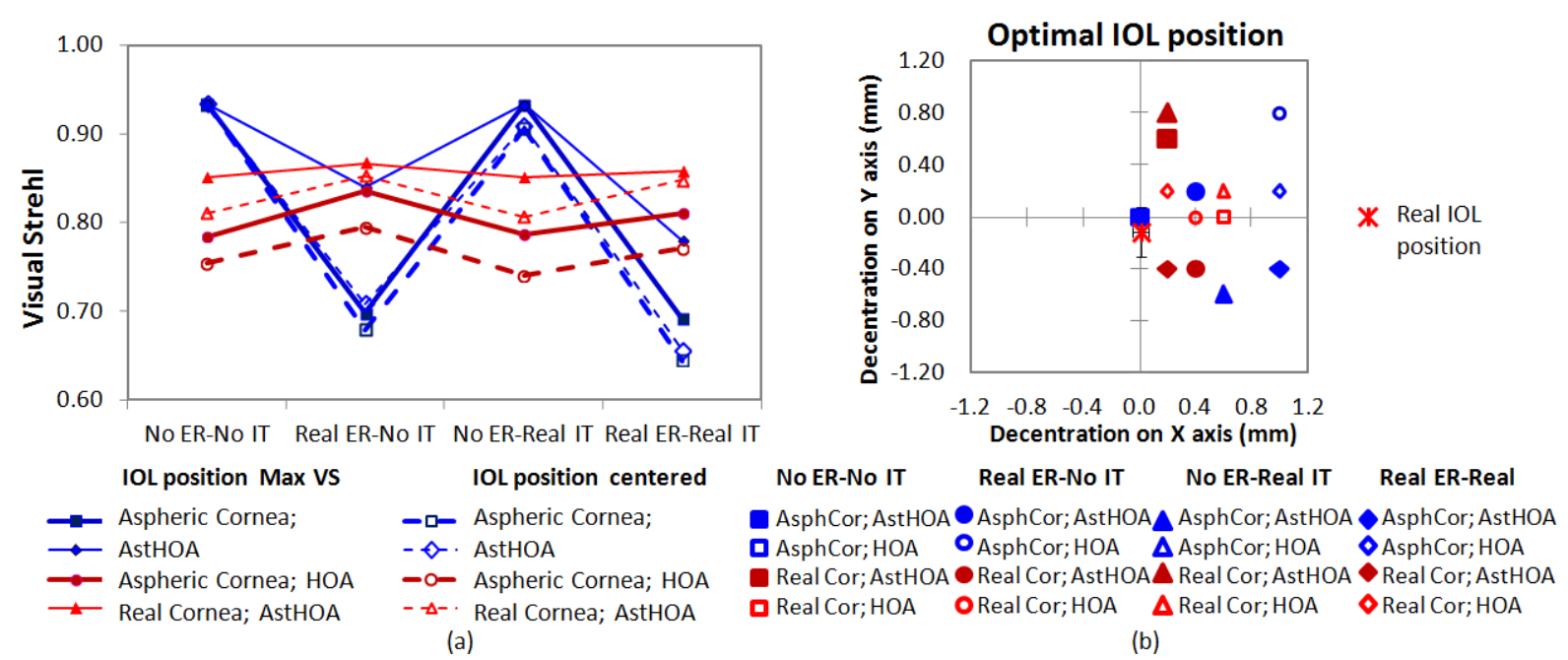

Figure 6.5 (a) the maximum VS achieved by optimally placing the IOL, assuming aspheric corneas (blue lines) or real corneal elevation maps (red lines), in comparison with a perfect centered IOL, for subject E\#1). Solid lines/solid symbols represent the max VS, while dashed lines/opened symbols represent the VS of centered IOL in the pupil center. Thicker lines/ symbols represent VS including astigmatism and HOA, while thinner lines/smaller symbols represent VS including HOA only. (b) Optimal IOL position (max VS) using aspherical and real corneal elevation maps with four conditions. Square, diamond, circle and triangle symbols represent the condition (without ER and IT) in the first column; with real ER and without IT in the second column; without ER but with real IT in third column; with real ER and real IT in the fourth column) respectively. Star symbol represent the real IOL position in E\#1. The color codes are the same in (a).

Figure 6.6 (a) shows the Maximum Visual Strehl (by optimizing IOL decentration), the Visual Strehl with the real IOL decentration, and assuming a centered IOL, obtained from patient specific eye models in all three patients, with their actual anterior and posterior corneal topographies and real eye rotation and IOL tilt. Data shown include Astigmatism and HOA (solid bars) and HOA only (shaded bars). The average deviations between max and real VS (Astigmatism +HOA) were $0.06,0.01$ and 0.08 (improved optical quality by $7.29 \%, 2.83 \%$ and $9.83 \%$ ) of E\#1 OD, E\#2 OS and E\#3 OS respectively. The average deviations between max and real VS (HOA only) were 0.01, 0.02 and 0.05 (improved optical quality by $1.05 \%, 3.23 \%$ and $5.35 \%$ ) of E\#1 OD, E\#2 OS and E\#3 OS respectively.

Figure 6.6 (b) shows the optimal IOL position (squares) and real IOL positions (circles), from estimations using astigmatism and HOA (closed) and HOA only (empty) for all three subjects. The optimal IOL positions (max VS including astigmatism and HOA) of E\#1 OD, E\#2 OS and E\#3 OS are $(0.20,0.40),(-0.20,-0.60)$ and $(-0.80,0.80) \mathrm{mm}$, while the optimal IOL positions (max VS including HOA only) are $(0.20,0.20),(-0.20,-1.00)$ and $(-0.60$, 1.00) $\mathrm{mm}$, on $\mathrm{x}$ and $\mathrm{y}$ axis, respectively, The deviation between the optimal IOL position of max VS (Astigmatism + HOA) and real IOL position of E\#1 OD, E\#2 OS and E\#3 OS are $0.21,0.81,0.85 \mathrm{~mm}$ on the horizontal axis respectively, and $0.28,0.41$ and $0.48 \mathrm{~mm}$ on the vertical axis respectively. The deviation between the optimal IOL position of max VS (HOA only) and real IOL position of E\#1 OD, E\#2 OS and E\#3 OS are 0.21, 0.81 and 0.65 $\mathrm{mm}$ on the horizontal axis respectively, and $0.08,0.81$ and $0.68 \mathrm{~mm}$ on the vertical axis respectively. 
OCT-based ray tracing on pseudophakic eyes to identify optimal IOL centration

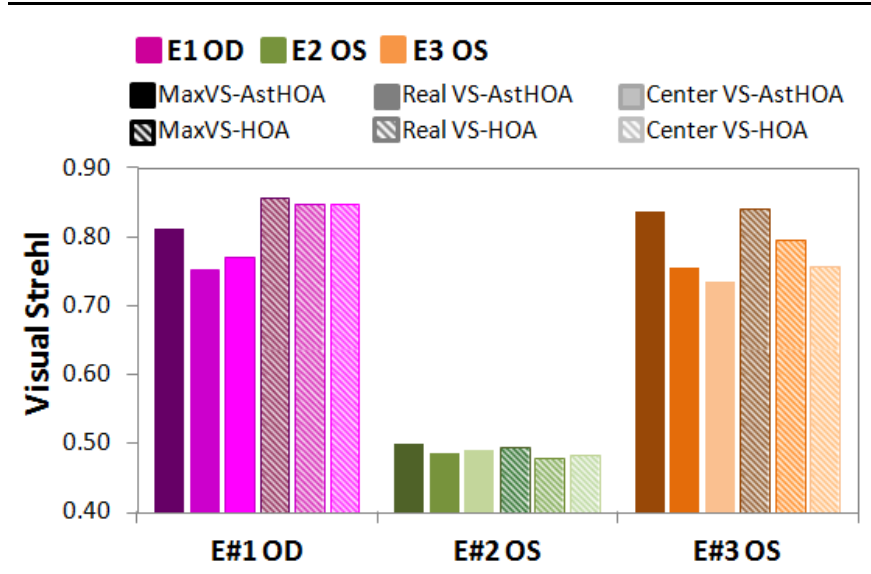

(a)

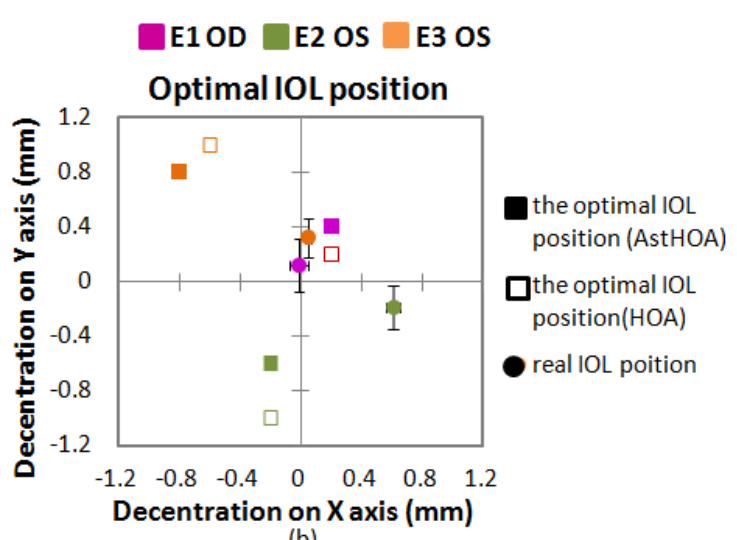

(b)

Figure 6.6 (a) the Maximum Visual Strehl (by optimizing IOL decentration), the Visual Strehl with the real IOL decentration, and assuming a centered IOL, obtained from patient specific eye models in all three patients, with their actual anterior and posterior corneal topographies and real eye rotation and IOL tilt. Data shown include Astigmatism and HOA (solid bars) and HOA only (shaded bars). (b) The optimal IOL position (squares) and real IOL positions (circles), from estimations using astigmatism and HOA (closed) and HOA only (empty) for all three subjects. The stander deviations of real IOL positions are from measurements.

\subsubsection{Interactions of eye rotation, IOL tilt and IOL decentration and corneal irregularities on astigmatism, trefoil and coma}

To further investigate interactive effects between misalignments and corneal irregularities as well as to understand the Zernike terms playing major roles in the improvement upon optimization, we evaluated RMS for astigmatism, coma and trefoil in similar conditions than those evaluated in 6.3.4 on patient E\#1 (no ER, no IT; Real ER, no IT; No ER, Real IT; Real ER and Real IT), as shown in Figure 6.5. The optimization was performed either considering astigmatism and HOA as well as HOA only.

As found in the VS analysis, aspheric corneas are more sensitive to the induction of astigmatism and coma in the presence of ER, while real corneas show less astigmatism and less coma for interactions of real ER and IOL tilt. According to Figure 6.6, with aspheric corneas, we found that optimizing IOL position of max VS (Astigmatism + HOA) improved the average RMS astigmatism (Figure 6.7 (a)) by $0.01 \mu \mathrm{m}$ by IT compared with zero IOL decentration, improved RMS coma by an average of $45.45 \%(0.01 \mu \mathrm{m})$, compared to centered IOL position, and by $53.20 \%(0.01 \mu \mathrm{m})$ compared between the optimal IOL position of max VS (HOA) and VS of a centered IOL shown in the Figure 6.7 (b). In the Figure 6.7 (c), RMS trefoil decreased $22.6 \%(0.01 \mu \mathrm{m})$ by ER, $87.93 \%(0.01 \mu \mathrm{m})$ by IT, and increased $65.84 \%(0.01 \mu \mathrm{m})$ compared between the optimal IOL position of max VS (Astigmatism + HOA) and centered IOL position, and the average RMS trefoil decreased $90.9 \%(0.01 \mu \mathrm{m})$ by three conditions (ER, IT, and ER\&IT) between max VS (HOA) and VS of a centered IOL.

In the computer eye model using real corneal elevations, we found the optimal IOL of max VS (Astigmatism + HOA) improved the average RMS astigmatism (Figure 6.7 (a)) by $50.82 \%(0.05 \mu \mathrm{m})$ with all conditions as compared to centered IOL position, and improved the average RMS astigmatism by $25.74 \%(0.01 \mu \mathrm{m})$ with IT, as compared between the 
optimal IOL position of max VS (HOA) and centered IOL position. The optimal IOL position of both max VS with/without astigmatism improved RMS coma by an average of $24.71 \%(0.01 \mu \mathrm{m})$, as compared centered IOL position. Spherical aberration (not shown) did not change with the presence of ER, IT, nor across IOL decentration.

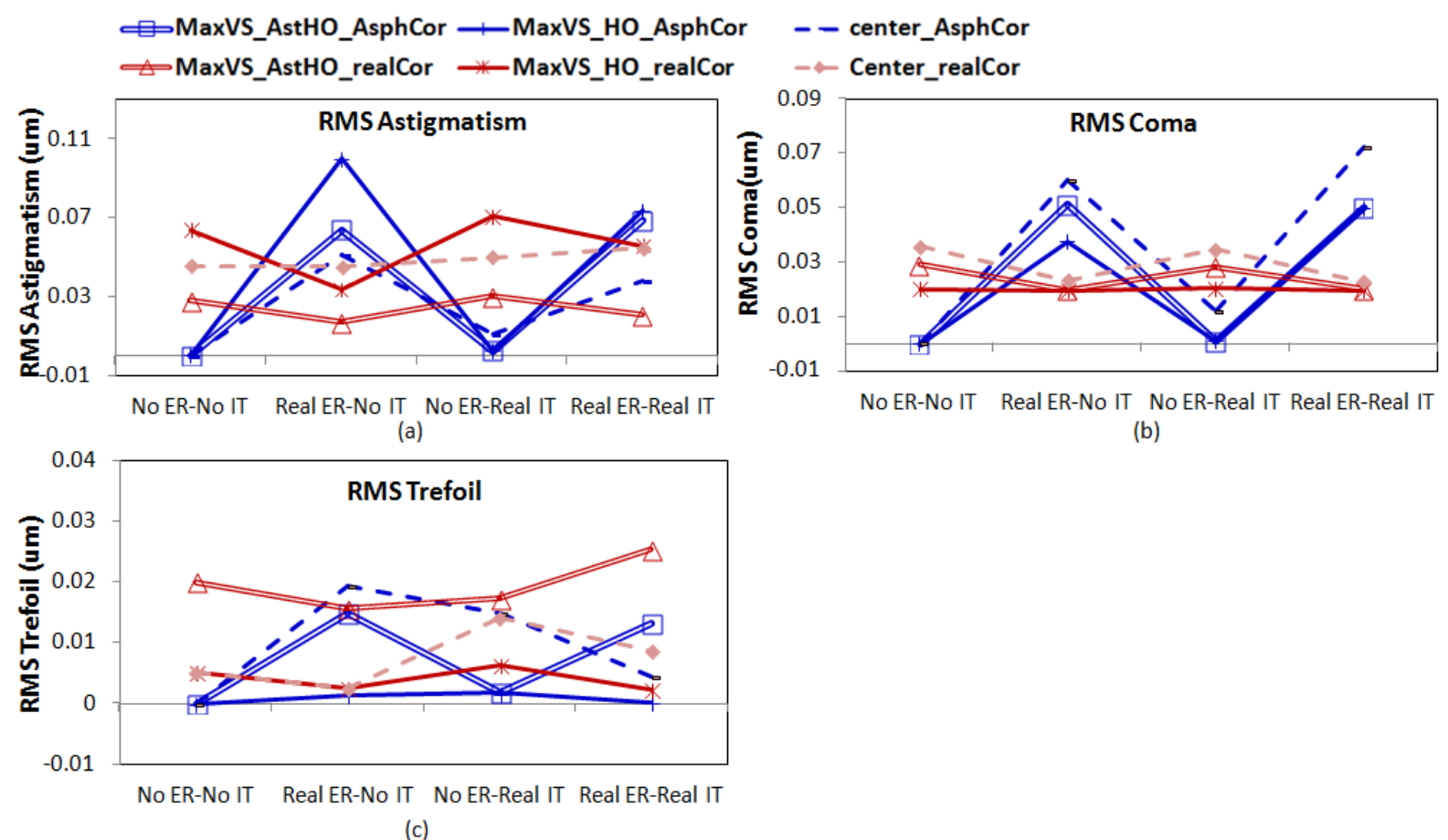

Figure 6.7 (a) RMS astigmatism; (b) RMS Coma and (c) RMS Trefoil changing with four conditions (no ER, no IT; Real ER, no IT; No ER, Real IT; Real ER and Real IT). Double solid line, single solid line and dashed line represent the max VS including astigmatism \& HO, the max VS including HOA only, and the VS of a centered IOL in the pupil center. The color codes are the same in Figure 6.4.

According to Figure 6.7, we found the optimal IOL position of max VS (astigmatism + HOA) improved the average RMS astigmatism (Figure 6.8 (a)) of E\#1 OD and E\#3 OS by $73.29 \%(0.03 \mu \mathrm{m})$, while the optimal IOL position of max VS (HOA) only improved RMS astigmatism of E\#3 OS by $26.82 \%(0.01 \mu \mathrm{m})$, with real ER and real IT, as compared to the centered IOL. The optimal IOL position of max VS (Astigmatism + HOA) improved RMS coma (Figure $6.8(\mathrm{~b}))$ by an average of $27.59 \%(0.01 \mu \mathrm{m})$ in all subjects, while the optimal IOL position of max VS (HOA) improved the average RMS coma of E\#1 OD and E\#3 OS by $50.31 \%(0.01 \mu \mathrm{m})$, as compared to the centered IOL position. In Figure 6.8 (c), the optimal IOL of max VS (Astigmatism + HOA) improved the average RMS trefoil by $16.48 \%(0.01 \mu \mathrm{m})$ in E\#2OS and E\#3OS, while the optimal IOL of max VS (HOA) improved the average RMS trefoil of all the subjects by $36.14 \%(0.01 \mu \mathrm{m})$ as compared to centered IOL position.

We found the optimal IOL position of max VS (astigmatism + HOA)/(HOA) improved the average RMS astigmatism among the pair of eye (E\#1 OD and E\#3 OS) (Figure 6.8 (a)) by $73.52 \%(0.04 \mu \mathrm{m}) / 55.01 \%(0.01 \mu \mathrm{m})$ respectively, with real ER and real IT, compared to the real IOL position. RMS coma (Figure 6.8 (b)) decreased by an average of $33.28 \%(0.01$ $\mu \mathrm{m}) / 38.46 \%(0.01 \mu \mathrm{m})$ among all subjects by the optimal IOL of max VS (astigmatism + 
HOA)/ (HOA), as compared to the real IOL position. Figure 6.8 (c) shows RMS trefoil decreased by $34.42 \%(0.01 \mu \mathrm{m})$ as average in all conditions by the optimal IOL position of max VS (HOA), as compared to the real IOL position.

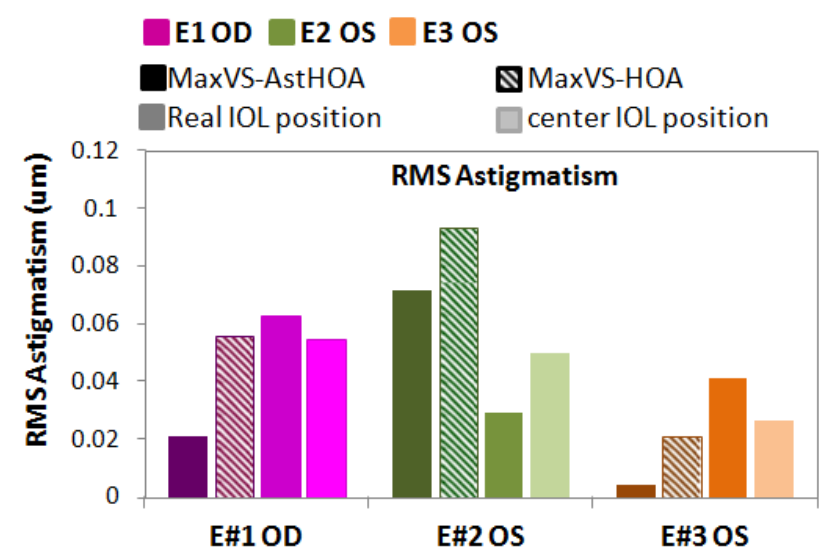

(a)

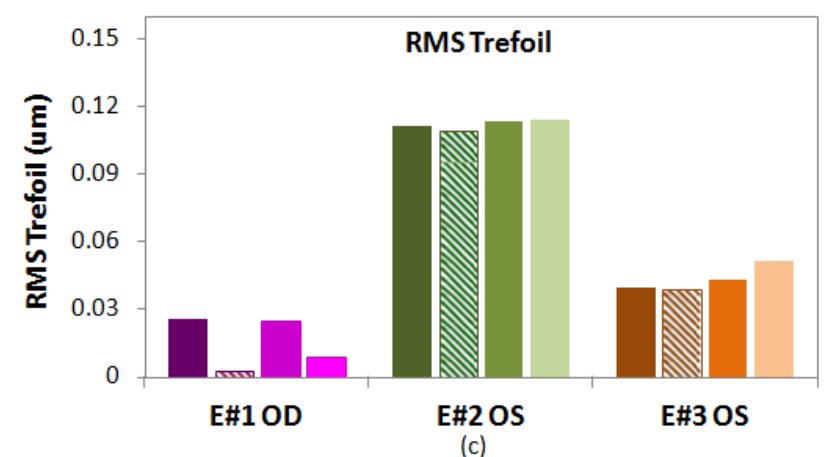

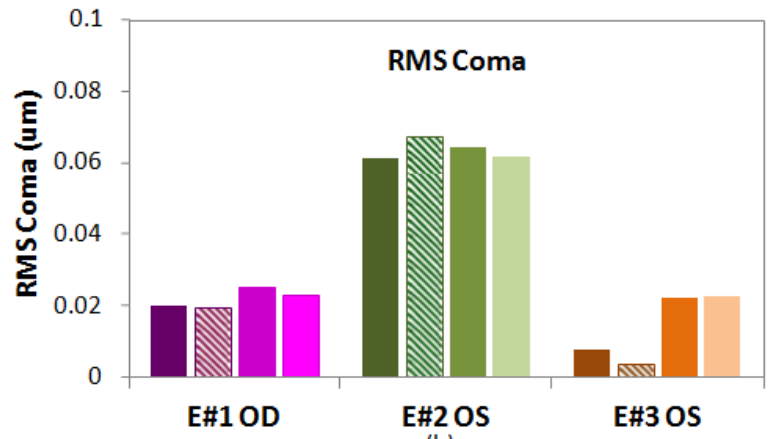

(b)

Figure 6.8 (a) RMS astigmatism; (b) RMS Coma and (c) RMS Trefoil with real ER and IT. Dark solid bars represent the optimal IOL position of max VS including astigmatism \& HOA, shade bars represent the optimal IOL position of max VS including HOA only, light solid bars represent the real IOL position in the three subjects, and lighter solid bars represent the IOL position centered in the pupil center. The three eyes are coded as the same in Figure 6.5.

\subsubsection{Residual defocus and residual astigmatism}

Results shown in Figures 6.4 have been obtained by optimizing VS through focus at each IOL position. Figure 6.9 showing VS pupillary maps with (i.e. equivalent to fine tuning the IOL power at each location) in the first row and without through-focus optimization (i.e. equivalent to keeping the IOL power constant) in the second row show that the optimal IOL position is independent of focus optimization.

We found that the maximum residual defocus difference was $0.85 \pm 0.16 \mathrm{D}$ in full VS pupillary map and that the difference in best focus for the real IOL position, centered IOL position and optimized IOL position was $0.10 \pm 0.02 \mathrm{D}, 0.09 \pm 0.03 \mathrm{D}$ and $0.16 \pm 0.03 \mathrm{D}$ for E\#1OD, E\#2OS and E\#3OS respectively in Figure 6.9. 


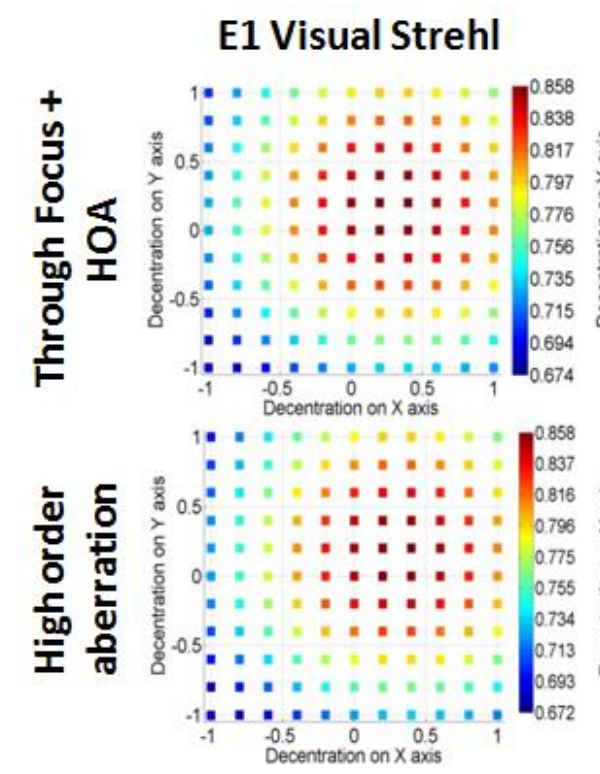

(a)

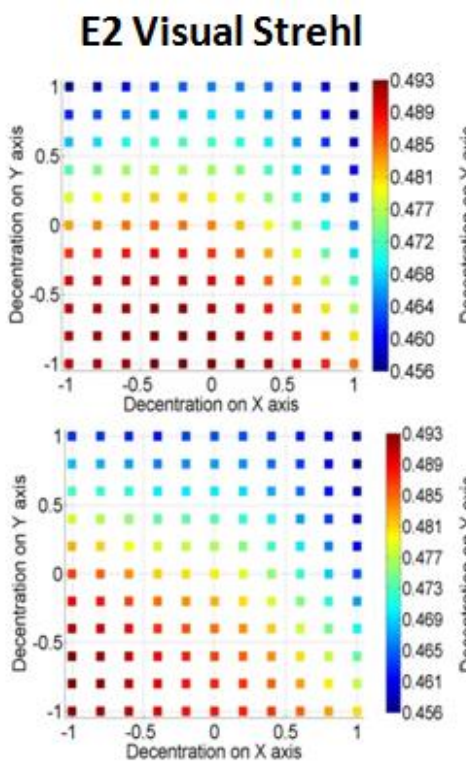

(b)

\section{E3 Visual Strehl}

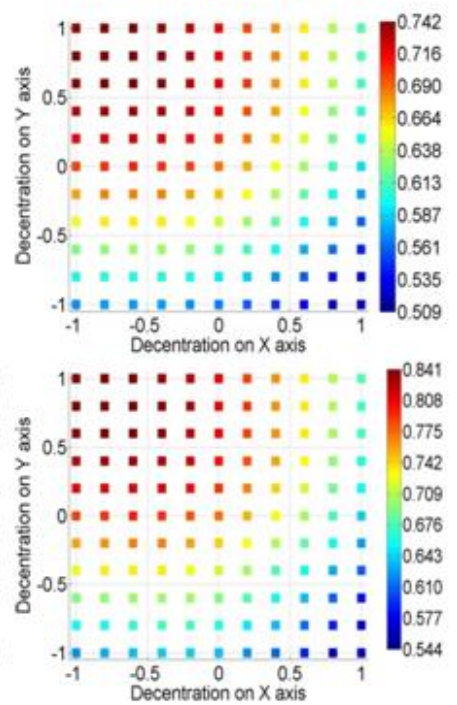

(C)

Figure 6.9 Visual Strehl (VS) of high order aberration through focus (from 3rd order Zernike terms with best focus) (in the first row) and without defocus (in second row) as a function of IOL position. (a) E1; (b) E2; (c) E3;

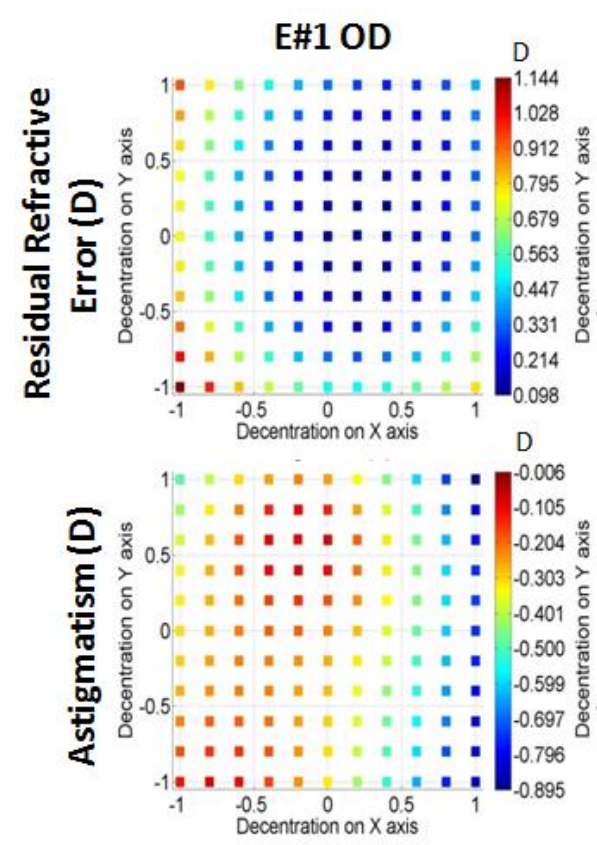

(a)

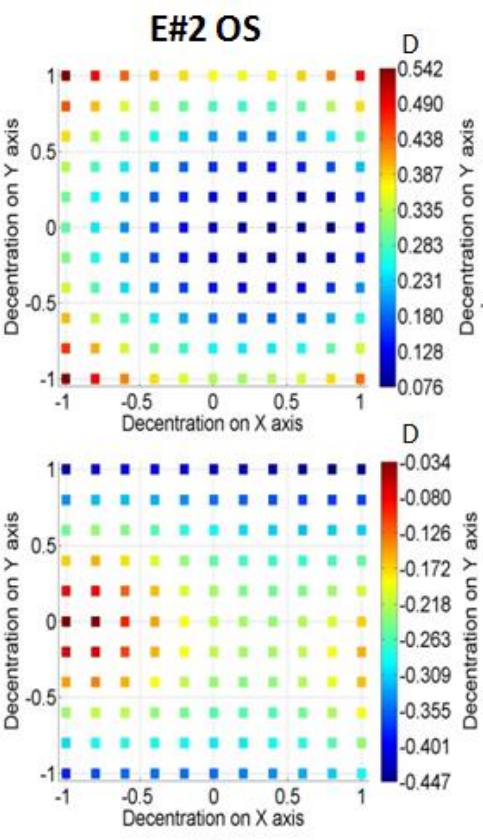

(b)

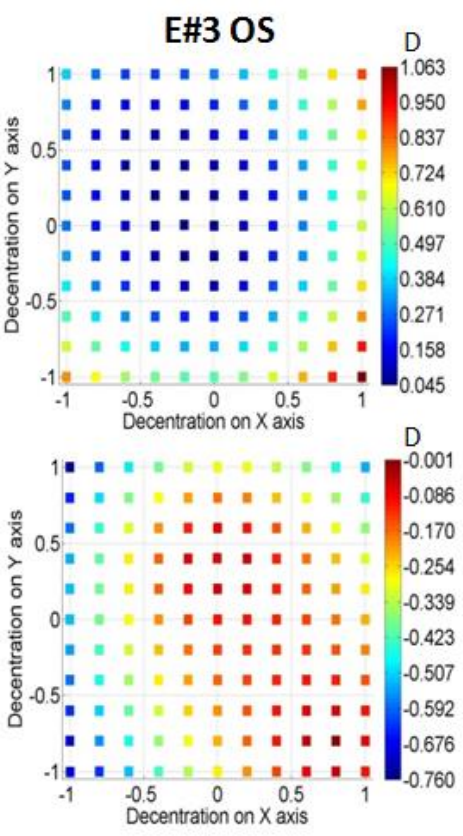

(c)

Figure 6.10 the residual refractive error (the first row) and astigmatism power (the second row) as a function of IOL positions of E\#1 OD (the first column), E\#2 OS (the second column) and E\#3 OS (the third column). (in D)

On the other hand, a comparison between the analysis for Astigmatism+ HOA and HOA only allows understanding the induction/correction of astigmatism by proper positioning of the IOL. The former assumes that astigmatism was not corrected by the implanted, and that the IOL position optimization may minimize astigmatism. The latter assume that the implanted IOL corrects for astigmatism at every location. We found that astigmatism 
changes by $0.69 \pm 0.13 \mathrm{D}$ across the entire VS pupillary map (average maximum to minimum across subjects), and that the difference in best focus for the real IOL position, centered IOL position and optimized IOL position of max VS (Astigmatism + HOA) was $0.25 \pm 0.06 \mathrm{D}, 0.20 \pm 0.08 \mathrm{D}$ and $0.13 \pm 0.12 \mathrm{D}$ for E\#1OD, E\#2OS and E\#3OS respectively in Figure 6.9. The power of astigmatism reduced when IOL located in the optimal of max VS including astigmatism and high order aberrations. Additionally, the optimal IOL position of max VS (Astigmatism + HOA) improved the more optical quality compare to the centered IOL position and the real IOL position. 


\subsection{Discussion}

Centration of surgical refractive procedures has been a matter of long debate, particularly in corneal surgery. In cataract surgery, the centration of the IOL has also been subject of numerous investigations. Some factors (e.g. incongruence between bag diameter and overall IOL diameter, a large capsulorhexis, asymmetrical capsule coverage, IOL placement in sulcus, and so on) may lead to large decentrations of high clinical relevance, malposition of an IOL is one of the reported leading causes for removal, exchange or repositioning of a posterior chamber IOL (Obstbaum and To 1989, Lyle and Jin 1992, Auffarth, Wilcox et al. 1995, Carlson, Stewart et al. 1998, Mamalis, Brubaker et al. 2008). On the other hand, investigations of the effects of IOL alignment on optical quality even in uneventful cataract procedures have paralled the increased design sophistication of stateof-the-art IOLs and have been enabled by new imaging technologies to accurate measure IOL alignment. Furthermore, OCT-based customized eye models which incorporate patient-specific information have been shown to accurately predict optical aberrations/optical performance in pseudophakic eyes (Sun, Pérez-Merino et al. 2016). In this chapter, we have used such models to understand the relative contributions of eye rotation, IOL tilt, corneal geometry and IOL decentration on optical quality in pseudophakic eyes. More specifically, we have demonstrated in three real eyes the possibility for improving optical performance by optimization of the IOL centration. As expected from the non-centered nature of the ocular components this optimal lateral position is not collinear with the pupil center, and in general, is not coincident with the actual IOL position. In some cases, we found the optimal IOL position of max VS including astigmatism and high order aberrations is in fact rather decentered $0.8 \mathrm{~mm}$.

We found that optimizing IOL decentration improved optical quality in real eyes by up to $9.83 \%(0.08)$ compared to the real IOL position (in E\#3OS) and was better by $15.01 \%$ (0.12) than if the IOL was centered at the pupil. The fact that real IOL decentrations (ranging from -0.19 to 0.61 ) exceeded optical quality with respect to that of no IOL decentration is in agreement with previous findings by Tabernero et al. (Tabernero, Piers et al. 2006) and Rosales and Marcos (Rosales and Marcos 2006) who reported individual performance of the IOL with decentration was better than that of a centered IOL, and by Eppig et al. (Eppig, Scholz et al. 2009), who reported that the best MTF of Invent ZO IOL and Aspira-aXa IOL are not in the zero decentration position.

Whether the relatively minor reported improvements potentially achieved by precise positioning of the IOL are worth the required haptic design and surgical sophistication remains to be seem. It is likely that a combination of customized IOL design, corrections of chromatic and monochromatic aberrations, and a higher tuning of the IOL power selection and astigmatism correction cooperate to further improvement of optical quality. We found that the IOL lateral shifts and the potential improvements in optical quality are highly subject dependent (ranging from 0.21 to $0.85 \mathrm{~mm}$ and $1.05 \%(0.01)$ to $9.83 \%(0.08)$ VS, respectively in our sample). Undoubtedly, the use of patient specific eye models allowing virtual ray tracing and evaluation of optical quality prior to IOL implantation will be key in selection of IOL that optimizes retinal image quality, including optimal IOL centration, and select the patient who will benefit from the improvements. 


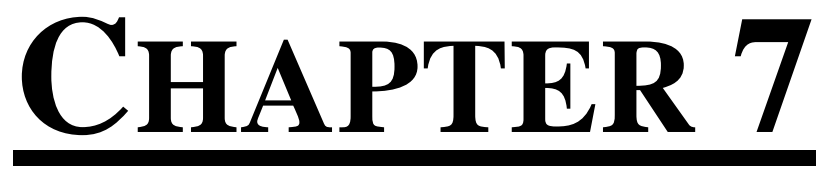

CONCLUSIONS 


\section{RESUMEN}

En esta tesis, hemos desarrollado una nueva metodología para medir el desalineamiento implantada de la IOL en pacientes con cirugía de cataratas a partir de imágenes de OCT en-face del segmento anterior. También hemos cuantificado la forma y la topografía 3D del cristalino del ojo in vivo, sus propiedades ópticas y sus cambios con la edad, que nos han permitido entender las fuentes de aberraciones oculares, compensaciones entre las superficies anterior y posterior del cristalino, y cambios estructurales con la edad., La metodología de OCT cuantitativa y modelos de ojo personalizados en pacientes han sido validados en pacientes operados de cirugía de cataratas, mediante comparaciones de las aberraciones simuladas y medidas en los mismos pacientes, y han permitido comprender la contribución relativa de los factores ópticos geométricos y quirúrgicos relacionados con la calidad de la imagen, como la identificación del centrado óptimo de la IOL. Estos son clave en los cálculos avanzados de la potencia de la IOL, la optimización de la selección individual de IOL o diseño de IOLs personalizados que puedan proporcionar una solución visual óptima al paciente. 


\subsection{Achievements:}

The main accomplishments of this thesis are:

1. We have implemented a comprehensive set of techniques for $3 \mathrm{D}$ quantitative biometry and IOL alignment based on OCT. An en-face OCT image Purkinje-like method to measure implanted IOL alignment in cataract surgery patients has been developed. With quantitative sOCT imaging of the pre- and post-operative anterior segment of the eye, this achievement appears essential to understanding the mechanisms by which current accommodative IOLs operate and advancing towards new developments. The method was tested on an in vitro artificial eye with known surface geometries at different orientations and demonstrated on an aging cataract patient in vivo.

2. Quantitative OCT with dedicated image processing algorithms allows quantification of the shape and topography of human lenses to study their optical properties and changes with age, and estimation of human crystalline lens volume, diameter and equatorial lens position, as validated from ex-vivo measurements. Quantitative OCT allows investigation of the geometrical changes of the crystalline lens with aging, contributing to the understanding of the optics and function of the lens in the eye. This quantitative information is key, among other things, to investigating the role of changes in the crystalline lens in presbyopia, and as inspiration for accommodating intraocular lenses aiming at the restoration of accommodation capacity. OCT lens imaging will increase accuracy in IOL power selection and be key in implantation of emerging accommodating IOLs.

3. Full 3D OCT based pseudophakic custom computer eye models (with individual data of corneal topography, IOL geometry, IOL tilt and decentration and eye rotation) have been developed and validations with experimental ocular aberrations from LRT have been achieved. The procedure includes the description of the surface shape with Zernike coefficients and wavefront analysis. The high similarity between optical and geometry-based predictions of high order aberrations in pseudophakic patients indicated that fully OCT-based ray tracing models are excellent platforms for optimizing IOL selection, and allow the understanding of the relative contribution of optical geometrical and surgically-related factors to image quality.

4. Full 3D OCT based pseudophakic custom computer eye models have been used to understand the relative contributions of eye rotation, IOL tilt, corneal geometry and IOL decentration on optical quality in pseudophakic eyes. We have demonstrated in three real eyes the possibility for improving optical performance by optimization of the IOL centration. We found that the IOL lateral shifts and the potential improvements in optical quality are highly subject dependent. The use of patient specific eye models allowing virtual ray tracing and evaluation of optical quality prior to IOL implantation will be key in selection of IOL that optimizes retinal image quality, including optimal IOL centration, and select the patient who will benefit from the improvements. 


\subsection{Conclusions}

This thesis presents a method to estimate tilt and decentration of IOLs based on optical coherence tomography (OCT) images. En-face OCT images show Purkinje-like images that correspond to the specular reflections from the corneal and IOL surfaces. Measurements of tilt and decentration of IOLs, along with the biometrical and anatomical parameters are of great value to evaluate optical quality in patients after cataract surgery, and to provide understanding of their relative role in optical degradation. The Purkinje-like method allows characterization of eye rotation, lens tilt and decentration, giving insights on the performance of implanted accommodative IOLs. The average amounts of tilt and decentration found in this study are $3.30 \pm 4.68 \mathrm{deg}$ and $0.16 \pm 0.16 \mathrm{~mm}$, respectively in eyes implanted with standard monofocal IOLs. These data, along with the biometrical data obtained from OCT, can be easily transferred into computer eye models for the evaluation of the contributing factors to optical degradation in eyes implanted with IOLs and the impact of IOL misalignment in particular.

Quantitative 3D OCT was used to measure surface topography of 36 isolated human lenses, and to evaluate the relationship between anterior and posterior lens surface shape and their changes with age. To our knowledge, this is the first comprehensive topographic study of the human lens with aging. All lens surfaces were fitted to $6^{\text {th }}$ order Zernike polynomials. Astigmatism was the predominant surface aberration in anterior and posterior lens surfaces (accounting for $\sim 55 \%$ and $\sim 63 \%$ of the variance respectively), followed by spherical terms, coma, trefoil and tetrafoil. The amount of anterior and posterior surface astigmatism did not vary significantly with age. In 13 of the 36 lenses the relative angle was $>45 \mathrm{deg}$, and 6 lenses had a relative angle $>70 \mathrm{deg}$. The average relative angle was larger for younger lenses ( $41.74 \mathrm{deg}$ for lenses $<50$ years) than for older lenses ( $28.1 \mathrm{deg}$ for lenses $\geq 50$ years), indicating a higher compensation of astigmatism between the lens surfaces at a younger age. The relative angle between anterior and posterior surface astigmatism axes was on average $36.5 \mathrm{deg}$, tended to decrease with age, and was $>45$ deg in $36.1 \%$ lenses. The anterior surface RMS spherical term, RMS coma and $3^{\text {rd }}$ order RMS decreased significantly with age. In general, there was a statistically significant correlation between the $3^{\text {rd }}$ and $4^{\text {th }}$ order terms of the anterior and posterior surfaces. This study was helpful for understanding the coordination of anterior and posterior lens surface geometries and their topographical changes with age and sheds light into the role of the lens in the optical properties of the eye and the lens aging mechanism.

Modeling the optics of an individual patient's eye and predicting the resulting optical performance addresses a current unmet need in visual optics. This thesis presents that full OCT-based pseudophakic custom computer eye models allow understanding of the relative contribution of optical geometrical and surgically-related factors to image quality, and are an excellent tool for characterizing and improving cataract surgery. We compared measured wave aberrations in pseudophakic eyes implanted with aspheric intraocular lenses (IOLs) with simulated aberrations from numerical ray tracing on customized computer eye models, built using quantitative 3D OCT-based patient-specific ocular geometry. Experimental and simulated aberrations show high correlation $(\mathrm{R}=0.93 ; \mathrm{p}<0.0001)$ and similarity (RMS for high order aberrations discrepancies within 23.58\%). These individual models have allowed evaluation of the effect of corneal aberrations, lens geometry, lens misalignment 
and angle lambda on the optical quality of eyes with implanted aspheric IOLs. Aspheric IOLs are able to correct the spherical aberration on average (although the exact amount of correction depends on the amount of corneal spherical aberration and several other biometric factors). Corneal aberrations play a major role in optical degradation in pseudophakic patients. The capability of OCT-based computer eye customized models to predict the measured aberrations post-operatively with a given implanted IOL opens the possibility to use those models as platforms to evaluate pre-operatively potential results with other IOLs, to optimize the selection of the IOL and improve optical outcomes.

OCT-based customized eye models which incorporate patient-specific information have been shown to accurately predict optical aberrations/optical performance in pseudophakic eyes. In this thesis, we have used such models to understand the relative contributions of eye rotation, IOL tilt, corneal geometry and IOL decentration on optical quality in pseudophakic eyes. More specifically, the three real eyes have been demonstrated the possibility for improving optical performance by optimization of the IOL centration. As expected from the non-centered nature of the ocular components this optimal lateral position is not collinear with the pupil center, and in general, is not coincident with the actual IOL position. In some cases, we found the optimal IOL position of max VS including astigmatism and high order aberrations is in fact rather decentered $0.8 \mathrm{~mm}$. The optimizing IOL decentration improved optical quality in real eyes by up to $9.83 \%(0.08)$ compared to the real IOL position (in E\#3OS) and was better by $15.01 \%(0.12)$ than if the IOL was centered at the pupil. Whether the relatively minor reported improvements potentially achieved by precise positioning of the IOL are worth the required haptic design and surgical sophistication remains to be seem. We found that the IOL lateral shifts and the potential improvements in optical quality are highly subject dependent (ranging from 0.21 to 0.85 $\mathrm{mm}$ and $1.05 \%(0.01)$ to $9.83 \%$ (0.08) VS, respectively in our sample). Undoubtedly, the use of patient specific eye models allowing virtual ray tracing and evaluation of optical quality prior to IOL implantation will be key in selection of IOL that optimizes retinal image quality, including optimal IOL centration, and select the patient who will benefit from the improvements.

In summary, this thesis developed a Purkinje-like methodology to measure IOL tilt and decentration in patients to help optimize IOL and haptic design. Quantifying the accurate biometry from sOCT measured data was our first task during the whole $\mathrm{PhD}$ research. Meanwhile, we built an aberration analysis model in Zemax to figure out the relationship between the geometry changes and optical aberration changes by using around 20 patients in our lab. Comparison between Profilometer and sOCT measurements in vitro should be helpful to increase the accuracy of IOL surface geometry. Corneal aberrations tend to increase after surgery, likely due to the incision effect. Total aberrations also increased by a lack of balance of the spherical aberration of the corneal by the spherical aberration of the intraocular lens. From clinic feedback, some factors (e.g. incongruence between bag diameter and overall IOL diameter, a large capsulorhexis, asymmetrical capsule coverage, IOL placement in sulcus, and so on) may lead to large decentrations of high clinical relevance, malposition of an IOL is one of the reported leading causes for removal, exchange or repositioning of a posterior chamber IOL. In order to identify optimal IOL centration, OCT-based ray tracing on a pseudophakic eye model was performed with all anatomical input data from the patient (anterior and posterior corneal elevation maps, IOL 
geometry, off-axis foveal angle, and IOL tilt), while decentration was changed from -1 to $+1 \mathrm{~mm}$ (in $0.2 \mathrm{~mm}$ steps) in a horizontal/vertical grid. Custom dynamic aberrometry in combination with sOCT technologies will be used to link structure and function in accommodation, both in young eyes and patients implanted with IOLs. 


\subsection{Summary}

In this thesis, we built new methodology to measure implanted IOL alignment in cataract surgery patients. We also quantified the shape and 3D topography of human lenses, their optical properties, and their changes with age and quantitative measurements of the crystalline lens based from Optical Coherence Tomography allow understanding the sources of ocular aberrations, predictions of the optical quality of the eye and improvement of cataract surgery. We also performed a series of measurements of cataract surgery patients and clinically viable measurements towards building full 3D OCT based pseudophakic custom computer eye models and validations with experimental ocular aberrations from LRT. Our researches help in understanding of the relative contribution of optical geometrical and surgically-related factors to image quality, such as identification optimal IOL centration using OCT-base ray tracing on pseudophakic eyes using analysis the optical quality effect with IOL decentration. These are key in advanced IOL power calculations and best individual IOL selection or customized IOL design based on the full-OCT based computer eye model for presbyopia correction that can provide an optimal visual solution. 


\subsection{Future work}

The implications and possible future directions of the work presented in this thesis include:

We have presented a comprehensive topographic study of the ex-vivo human lens with aging, which could be used in a ray tracing analysis for lens GRIN distribution. Further studies on a larger population of different ages and/or refractive profiles of in-vivo crystalline lens topography will allow insights on the role of the crystalline lens on the agedependent changes of the eye's optics, myopia development, ocular astigmatism and accommodation. This thesis presents that astigmatism and high order terms may be considered when trying to predict optical quality of a phakic eye at an individual level, based on anatomical information.

Combined measurements of wavefront aberrations and 3D corneal and crystalline lens surface shape in vivo provided a deeper understanding of the relative contributions of corneal and lens surfaces themselves to the aberrations and allowed realistic individual simulations of ocular properties, such as ocular aberrations, by incorporating a patient's based eye biometry. This future work will allow evaluation of the contribution of individual optical components (such as, corneal and crystalline lens surfaces, GRIN) and their alignment to optical degradation. Furthermore, more realistic and advanced customized computer eye models will potentially provide additional information including residual postoperative refractive error, chromatic aberration, IOL manufacturing imperfections, ocular pathology, and scatter of optical media.

The results of monitoring IOL misalignment can establish the basis for future research line directions of dynamic changes of lens geometry and misalignment with accommodation, in relationship with the dynamic changes of ocular aberrations, which would shed light onto the mechanism of lenticular accommodation and presbyopia. Application of OCT (direct visualization) and LRT aberrometry (objective accommodative response) in the study of patients implanted with accommodative IOLs (A-IOL) will be essential to evaluate the mechanism of action of the A-IOL and its final positioning in different accommodative demands. The future application of this technique in the measurement of real crystalline lens GRIN presents important implications in the study of the mechanisms of accommodation and presbyopia.

OCT-based custom computer eye models will also be applicable to the optimization of the IOL power calculation, using virtual ray tracing on these eyes. Ray tracing IOL power calculation will overcome many limitations of current IOL power selection techniques. These are typically based on regression models obtained for a large patient population, as opposed to the individualized approach based on customized eye models. Pre-operative measurements of anterior and posterior corneal surfaces will overcome the limitations of the standard IOL power calculations which only make use of anterior corneal keratometry and the so-called keratometric index. Clinical evaluation of the advanced IOL design is required to verify accurate predictions of improved quality of vision and fewer complications after cataract surgery. OCT-based customized computer eye models will be excellent predictive tools of the optical quality in pseudophakic eyes, allow understanding of the factors that contribute to optical degradation and hold promise to become a primary 
tool to optimize the selection of the IOL to be implanted in a cataract procedure. Similar to the comparisons between the measured and simulated high order aberrations addressed in the current study, validations of the IOL power selections will involve comparisons of measured and predicted aberrometric defocus and/or refraction.

The future studies will allow improved understanding of the accommodation mechanism by relating morphological changes in the crystalline lens with changes in the ocular aberrations and accommodative response, addressing the basis for the fluctuations of accommodation, and unraveling the influence of aberrations on the accommodation dynamics. The conclusions will have impact on the mechanism of action and limitations of current accommodative IOL designs, and optical and structural properties of eyes implanted with A-IOLs as a function of accommodative demand from OCT and aberrometric measurements by the future study of optical simulations of performance of A-IOLs from customize eye modeling and mechanical and optical testing of new A-IOL prototypes. 


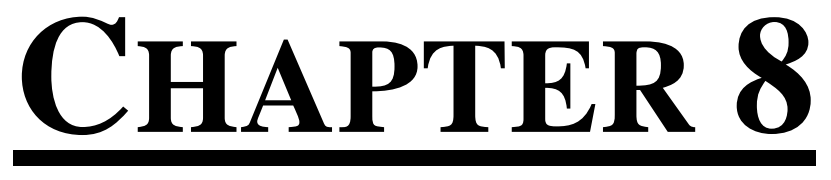

PUblications AND

ACTIVITIES DURING THE

PHD PERIOD 


\subsection{Publications}

1. M. Sun, P. Pérez-Merino, E. Martinez-Enriquez, M. Velasco-Ocana and S. Marcos. "Full 3D OCT-based pseudophakic custom computer eye model." Biomedical Optics Express 7(3): 1074-1088. (2016).

2. $\quad$ E. Martinez-Enriquez, P. Pérez-Merino, M. Sun, S. Durán-Poveda, I. JiménezAlfaro, S. Marcos. "Full shape crystalline lens quantification from 3-D OCT images and its application to predict the post-operative IOL position." ARVO E-abstract 2686338 (2016).

3. S. Marcos, E. Martinez-Enriquez, M. Sun, P. Pérez-Merino, Velasco-Ocana M., "3D OCT ocular biometry." XXII Biennial Meeting of the International Society for Eye Research ISER • Abstract: A-817-0005-00262. (2016)

4. E. Martinez-Enriquez, M. Sun, M. Velasco-Ocana, J. Birkenfeld, P. PérezMerino, and S. Marcos. "Optical Coherence Tomography Based Estimates of Crystalline Lens Volume, Equatorial Diameter, and Plane Position." Invest Ophthalmol Vis Sci 57(9): OCT600-610 (2016).

5. S. Marcos, E. Martinez-Enriquez, P. Pérez-Merino, M. Velasco-Ocana, and M. Sun, "Unraveling eye crystalline lens optics, structure and function," in Latin America Optics and Photonics Conference, (Optical Society of America), paper LTh2C.1. (2016)

6. S. Marcos, P. Pérez-Merino, E. Martinez-Enriquez, M. Sun, and M. Velasco, "Advances in Anterior Segment OCT: Crystalline and Intraocular Lens Applications," in Frontiers in Optics 2015, OSA Technical Digest (online) (Optical Society of America), paper FM2F.1. (2015)

7. M. Sun, P. Pérez-Merino, S. Durán-Poveda, Ignacio Jiménez-Alfaro, S. Marcos. "OCT-based ray tracing on pseudophakic eyes to identify optimal IOL centration." Invest. Ophthalmol. Vis. Sci.;56(7):2972. (2015)

8. M. Sun, J. Birkenfeld, A. de Castro, S. Ortiz and S. Marcos. "OCT 3D surface topography of isolated human crystalline lenses." Biomed Opt Express 5(10): 3547-3561. (2014).

9. M. Sun, A. de Castro, S. Ortiz, P. Pérez-Merino, J. Birkenfeld and S. Marcos. "Intraocular lens alignment from an en-face optical coherence tomography image Purkinje-like method." Optical Engineering 53(6): 061704-061704. (2014).

10. S. Marcos, S. Ortiz, P. Pérez-Merino, M. Velasco, M. Sun, J.Birkenfeld, S. Durán-Poveda, I. Jimenez-Alfaro. "Three-dimensional biometry and alignment in eyes implanted with Accommodative IOLs as a function of accommodative demand. " Invest. Ophthalmol. Vis. Sci. ;54(15):382 (2013). 


\subsection{Talks and posters presented in the conferences}

1. "OCT-based ray tracing on pseudophakic eyes to identify optimal IOL centration", poster presentation, International OSA Network of Students Conference, Valencia, Spain, September, 2015.

2. "OCT-based ray tracing to identify optimal IOL centration", oral presentation, $2^{\text {nd }} \quad$ IOSA Scientific Seminar series, Madrid, Spain, June, 2015.

3. "OCT-based ray tracing on pseudophakic eyes to identify optimal IOL centration", poster presentation, The Association for Reasearch in Vision and Ophthalmology, Denver, United States of America, May, 2015.

4. "Monitoring lens accommodations with imaging techniques", oral and poster presentations, $4^{\text {th }}$ Optical and Adaptational Limits of Vision Annual meeting, Murcia, Spain, January, 2015.

5. "Full OCT-based pseudophakic custom computer eye model", oral presentation, Visual and Physiological Optics conference, Wroclaw, Poland, August, 2014.

6. "Monitoring lens accommodations with imaging technique", oral and poster presentations, $3^{\text {rd }}$ Optical and Adaptational Limits of Vision Annual meeting, Stockholm, Sweden, May, 2014,

7. " OCT 3D surface topography of isolated human crystalline lenses", poster presentation, The Association for Research in Vision and Ophthalmology, Orlando, United States of America, May, 2014.

8. "Monitoring lens accommodations with imaging technique", oral presentation, $2^{\text {nd }}$ Optical and Adaptational Limits of Vision Annual meeting, Santorini, Greece, July, 2013.

9. "Role of Ocular Aberrations on Accommodation Dynamics", oral presentation, Mid-term review meeting, March, Brussels, Belgium, March, 2013.

10. "Monitoring lens accommodations with imaging technique", oral and poster presentations, $1^{\text {st }} \quad$ Optical and Adaptational Limits of Vision Annual meeting, Madrid, Spain, September, 2012.

11. "Monitoring lens accommodations with imaging technique", oral presentation, Optical and Adaptational Limits of Vision, Tübingen, Germany, March, 2012. 


\subsection{Visits and Stays in Research Institutions, Hospital and Companies}

March $5^{\text {th }}$ to $9^{\text {th }}, 2012$, at Institute for Ophthalmic Research, in Tübingen, Germany

1. Participating the training courses (Essentials of Project Management I+II, Ethics in Reasearch, and the Craft of Scientific Presentations I+II)

2. Visiting Prof. Frank Schaeffel 's Laboratory of University Hospital Tuebingen in section of Neurobiology of the Eye Centre for Ophthalmology

May $29^{\text {th }}$ to $30^{\text {th }}$, 2012, at Rodenstock GmbH in Munich, Germany

3. Getting insight into industrial research and development from basics to product development of individual progressive addition lenses.

August $20^{\text {th }}$ to $22^{\text {th }}$, 2012, at University College Dublin (UCD), Dublin, Ireland

4. Visiting Prof. Brain Vohnsen's Laboratory of the Advanced Optical Imaging group in School of Physics, Belfield Campus

July $7^{\text {th }}$ to $17^{\text {th }}$, 2013, at University of Crete faculty of medicine in Heraklion, Crete, Greece

5. Participating in preoperative examination of partients scheduled for cataract extraction in the premises of University Hosptal of Heraklion

6. Visiting Prof. Ioannis Pallikaris and Prof. Harilaos Ginis's Laboratory of Optics and Imaging in the premises of Departement of Medicine

7. Visiting the Operating Room of the University hospital, observing operations for cataract extraction with phacoemulsification and presbyopia correction.

May $19^{\text {th }}$ to $21^{\text {st }}$ and $26^{\text {th }}$ to $27^{\text {th }}$, 2014, at KTH Royal Institute of Technology, in Stockholm, Sweden

8. Participating the training courses (Statistic Course I+II, Intellectual Property Rights, Personal Leadership, Managing Stress, Introduction into peer review and Psychophysics)

9. Visiting Prof. Linda Lundstrom and Prof. Peter Unsbo's Laboratory of Applied Physics/ Biomedical and X-Ray Physics in AlboNova University center

January $21^{\text {st }}$ to $23^{\text {th }}$, 2015, at Universidad de Murcia, in Murcia, Spain

10. Visiting Prof. Pablo Artal's Optics Laboratory at the University of Murcia in the Research Center for Optics and Nanophysics 


\section{FIGURE LIST}

\section{Chapter 1}

Figure 1.1 (a) The general layout of a normal young eye: cornea, iris, pupil, crystalline lens, retina, macula, fovea and blood vessels; (b) The limitation of temporal and nasal field of vision (source: Book: Optics of the Human eye).

Figure 1.2 Histological section of a human cornea (source: www. missionforvisionusa. org).

Figure 1.3 Types of astigmatism as determined by the orientation of the principal meridians and the orientation of the correcting cylinder axis.[Paul Riordan-Eva et al., 2003, source:http://www.oculist.net/others/ebook/generalophthal/server-java/arknoid/amed/ vaughan/ co_chapters/ ch020/ch020_print_01.html]

Figure 1.4 The structure of the Human Lens. Suture and Equator are anatomical terms in ophthalmology. Suture means the seams of the lens. The suture patterns become more complex as more layers of lens fibers are added to the outer portion of the lens. Equator means the edge of the largest portion of the lens (similar to the equator on a globe) (source: http://photobiology.info/Roberts.html)

Figure 1.5 Quantitative elevation maps of the posterior lens surface for an in vitro 65-year donor lens. Left panel: Measurements with the posterior surface of the lens facing the OCT beam ("posterior up"); Middle panel: Measurement of the posterior surface of the lens viewed through the anterior surface of the lens, and no optical distortion correction (simple division by the index of refraction); Right panel: Measurement of the posterior surface of the lens viewed through the anterior surface of the lens, after application of optical distortion correction. Maps are fit relative to the best fitting sphere with Zernike polynomials. $\mathrm{R}$ is the radius of curvature of the best fitting (Ortiz, Perez-Merino et al. 2012).

Figure 1.6 Lens model and definition of some parameters of interest. DIA: Diameter; PS: Pupil size; ID: Diameter in the intersection; AL: Anterior lens; PL: Posterior lens; EPP: Equatorial plane position; $\alpha$ : Diameter that defines the central portion of the lens; PROP: Proportion of the diameter in the intersection. $\rho$ : Part of the central portion taken to fit every lens side. (E. Martínez-Enriquez 2016)

Figure 1.7 Drawn definitions of optical axis, pupillary axis, line of sight, visual axis (Arbelaez, Vidal et al. 2008)

Figure 1.8 Diagrams of refractive error (Normal Eye, Myopia, Hyperopia, and Astigmatism)

Figure 1.9 Perceived image with defocus, astigmatism, and high order aberration such as spherical aberration, coma, trefoil and random aberration vs. light field display (source: http://www.zdnet.com/article/researchers-eye-vision-correcting-displays-for-devices/)..... 35

Figure 1.10 A quarter section through the anterior segment 
Figure 1.11 The modern technique of Phacoemulsification which consists in the emulsification (dissolve and aspire) of the crystalline lens inside the eye and the introduction of a foldable lens through an average 2-mm incision.

Figure 1.12 Schematic representations of (a) TD-OCT and (b) FD-OCT. (Bezerra, Costa et al. 2009)

Figure 1.13 Block diagrams of the OCT methods with the use of Fourier-domain detection: (a) SD-OCT, (b) SS-OCT. (Wojtkowski 2010). 46

Figure 1.14 Hartmann-Shack system in optometry: a laser creates a virtual light source in the retina. The lenslet array creates spots in the sensor according to the wavefront coming out of the eye. The local tilt of the wavefront across each lens can be calculated from the position of the focal spot on the sensor. (Source: https://en.wikipedia.org/wiki/Shack\%E2\%80\%93Hartmann_wavefront_sensor).....

Figure 1.15 (a) Scheme of H-S. (b) Scheme of LRT (Navarro and Moreno-Barriuso 1999)

Figure 1.16 Schematic of Gullstrand-Emsley eye model (cardinal points at reasonable location; $10.9 \mathrm{D}$ accommodated forms; anterior lens surface moved $0.4 \mathrm{~mm}$ with more curved anterior lens surface).

Figure 1.17 Models of gradient index with internal optical structure (a) Gullstrand 1909 (b) Liou \& Brennan 1997 (c) Navarro et al. 2007 (d) Alberto et al. 2010 (Crystalline lens GRIN with age function)

\section{Chapter 2}

Figure 2.1 Photograph of the actual custom Spectral-Domain Optical Coherence Tomography device at the VioBio lab (Instituto de Óptica, "Daza de Valdés")....

Figure 2.2 The schematic diagram of the custom SD-OCT set-up, components: Low coherence broad band spectrum source ; Collimating lens; Beam Splitter 1; Reference mirror; Beam splitter 2; Badal system; Picoprojector and external screen; Scanning galvanic mirror; Objective lens; Condensing lens; Grating; Line-scan CMOS camera

Figure 2.3 Map of residual fan distortion before (a) and after (b) correction, obtained from a flat optical surface with the OCT system of the study. Reproduced from (Ortiz, Siedlecki et al. 2010, Ortiz, Siedlecki et al. 2011) (c) Illustration of the effect of the 3D distortion correction on the anterior segment surfaces (all surfaces are corrected axially from refractive index. Green: corrected volumes; red: uncorrected volumes). Reproduced from (Ortiz, Siedlecki et al. 2009, Perez-Merino, Velasco-Ocana et al. 2015).

Figure 2.4 Illustration of pupil centration as reference across: A: anterior and posterior cornea, iris; B: iris and anterior lens; C: pupil, anterior lens and posterior lens in effective area within the pupil; D: merged volumes of anterior segments. Blue and red points present anterior and posterior cornea surfaces respectively; Light blue and black points present anterior and posterior lens surfaces respectively; Yellow, green and pink points present iris.

Figure 2.5 Coefficients of the Zernike polynomials up to the 6th radial order with 28 terms using the Optical Society of America (OSA) standard (Thibos, Applegate et al. 2002) ..... 68 
Figure 2.6 Quantitative anterior and posterior cornea and crystalline lens topography, after full distortion correction. Maps are Zernike fits to the elevation maps, relative to the best fitting sphere. (Ortiz, Perez-Merino et al. 2012)

Figure 2.7 (a) Three-dimensional (3D) optical coherence tomography (OCT) image; (b) enface OCT image showing the pupil merging, the locations of PI, PIII, and PIV in red and pupil center in yellow; (c) PI (left), PIII (center), and PIV (right) in OCT B-Scan images. 70

Figure 2.8 (a) USAF 1951; (b) OCT fundus image in SOCT data process program ; (c) OCT fundus image rotated 90 deg counterclockwise saved as JPG image; (d) Rescaling the height and width of JPG from 300 A-scans and 50 B-scans OCT image as taken during OCT measuremnts $15 \times 7 \mathrm{~mm}$ measurement setting; (e) Image fundus rotation 90 degree clockwise and flipping around horizontal axis to have real en-face OCT image;(f) The definition of global coordinates of eye rotation, IOL tilt and decentration based on en-face OCT image; (g) Nomenclature and sign conventions for eye rotation, IOL tilt and decentration looking into the human eyes, as seen by the observer. The coordinate is the same as (Rosales and Marcos 2006).

Figure 2.9 (a) Custom Laser Ray Tracing setup at the VioBio lab (Instituto de Óptica, "Daza de Valdés"). Retinal camera captured the light reflected back from the retina. Pupil camera captured the corresponding image of the eye's pupil simultaneously with the retinal spots on the retinal CCD. (b) Scheme of the custom-LRT modified from (Perez-Merino, Birkenfeld et al. 2014) (red solid line: illumination channel; dark blue dash line: Pupil and retinal channel.) .74

Figure 2.10 (a) Snapshot of the control program developed by Carlos Dorronsoro (Top right and bottom left are the pupil and retinal images, respectively); (b) Example of aerial images obtained from LRT using green and IR light in Panels A and B. Panel C shows the corresponding spot diagram. Crosses and circles represent green and IR illumination respectively. (Llorente, Diaz-Santana et al. 2003) .75

Figure 2.11 (a) commercial Pentacam Scheimpflug topographer (Oculus Optikgeräte GmbH, Wetzlar, Germany) at the VioBio lab (Instituto de Óptica, "Daza de Valdés") (b) interface of commercial Pentacam Scheimplug topographer .76

Figure 2.12 Corneal elevation maps obtained in 4 eyes obtained from different instruments (relative to the best fitting sphere). $\mathrm{R}=$ radii of curvature of the best fitting sphere (from fits to sphere quadrics), modified from (Ortiz, Siedlecki et al. 2011). .77

Figure 2.13 (a) Commercial non-contact profilometry (Sensofar, PLu2300,Barcelona, Spain) (b) profilometry raw data with tilt and noise (c) quantified anterior and posterior surfaces of intraocular lens with customized algorithm .78

Figure 2.14 (a) Photograph and (b) schematic diagram of the physical model eye developed for this study. A PMMA contact lens simulates the cornea. The IOLs are positioned with an XYZ micrometer stage and rotational stage (de Castro, Rosales et al. 2007). .79

Figure 2.15 Illustration of the computation of aberrations using quantitative OCT geometrical data in a customized computer pseudophakic eye model.

Figure 2.16 (a) Example of RMS radius and max GEO radius report of spot diagram from 14 to -14 degree field angle around horizontal axis with three wavelengths (Blue, green and red present the wavelengths of $486.1 \mathrm{~nm}, 587.6 \mathrm{~nm}$ and $656.3 \mathrm{~nm}$, respectively) in Zemax; Circle in white is Airy Disk; (b) Example of spots diagram images obtained from LRT at 543 nm wavelength . Error! Bookmark not defined.

Figure 2.17 (a) Optical Distance Errors; (b) Wavefront Error; (c) Ray Errors .83 
Figure 2.18 Wavefront aberration: 1st order aberrations: (a) Vertical Tilt (b) Horizontal Tilt; 2nd order aberrations: (c) Oblique Astigmatism (d) Vertical Astigmatism (e) Defocus; 3rd order aberrations: (f) Vertical Coma (g) Horizontal Coma; 4th order aberration: (h) Spherical Aberration

Figure 2.19 Process steps of patients' measurement: Aberrometry, Pentacam, IOLmaster and Optical Coherence Tomography

Figure 2.20 Solid Model 3/4 draw section of full-OCT based customized eye model. Corneal Surfaces: OCT-based 6th order Zernike Surface Sag on best fitting sphere; IOL Surfaces: OCT-based geometry (aspheric surfaces); Biometry: OCT-based corneal thickness and ACD; axial length from IOLMaster; Alignment: IOL tilt \& decentration from Purkinje-like OCT; Angle lambda from eye rotation.

Figure 2.21 3D layouts of simulations in ZEMAX (a) PI (b) PIII (c) PIV 89

Figure 2.22 (a) Real IOL decentration in patient's eye after cataract surgery (b) solid model $3 / 4$ draw sections of customized eye model with simulated decentrations ranging is from -1$1 \mathrm{~mm}$ in $0.1 \mathrm{~mm}$ steps for $5 \mathrm{~mm}$ pupil diameter along horizontal and vertical meridians, with reference to the pupil center.

Figure 2.23 Illustration of OCT automatic image processing (Ortiz, Siedlecki et al. 2010, E. Martínez-Enriquez 2016)

Figure 2.24 Illustrated algorithm of OCT 3D surface topography of isolated human crystalline lenses.

\section{Chapter 3}

Figure 3.1 Experimental data vs. nominal data in the artificial eye model (a) IOL tilt (in air); (b) IOL tilt (in water); (c) IOL decentration (in air); (d) IOL decentration (in water) ...... 106

Figure 3.2 (a) IOL tilt and (b) IOL decentration in the 5 eyes studied. Solid symbols represent data obtained using the Purkinje-like OCT-based method and open symbols represent data obtained directly from distortion-corrected 3D OCT images (Ortiz, Perez-Merino et al. 2013). Square, rhombus and round represent right eyes (OD) while triangle and rectangle represent left eyes (OS). Each color (color online only) represents a different eye. Error bars stand for standard deviations of repeated measurements. 106

Figure 3.3 Correlation between data obtained from Purkinje-like and OCT methods for IOL tilt $(a, b)$ and decentration $(c, d)$ in all 5 eyes of the study..... 108

Figure 3.4 Bland-Altman plot-differences between Purkinje-like and OCT estimates of tilt (a) and decentration (b). Horizontal lines represent the mean difference (-0.17 deg and -0.09 $\mathrm{mm}$, respectively) and $95 \%$ limits of agreement.

Figure 3.5 Experimental en-face OCT image (a) and simulated (b) PI, PIII and PIV, for S\# 8 OS 


\section{Chapter 4}

Figure 4.1 Upper panels: OCT images corresponding to the anterior-up position of one of the crystalline lenses imaged, with (a) OCT focused on the anterior surface and (b) OCT focused on the posterior surface and the image of the cuvette. The detected surfaces are marked in blue (anterior surface), red (posterior surface) and green (cuvette). Lower panels: 3D OCT data from images of the crystalline lens with (c) the anterior surface up and (d) the posterior surface up. The blue and red points correspond to the segmented anterior and posterior surfaces of the lens, respectively. The green points and the black points correspond to the cuvette imaged through the lens, therefore distorted by the lens, and the cuvette without distortion respectively.

121

Figure 4.2 Lens surface elevation maps for all lenses, ordered by age. By convention, the maps are aligned so that the steepest meridian of the anterior lens surface lies on the vertical axis. Anterior and posterior images are shown as mirrored on the vertical axis. Asterisks indicate pairs of lenses from the same donor.

Figure 4.3 (a) Radii of curvature of anterior and posterior lens surfaces at their corresponding steepest meridian as a function of age (b) Radii of curvature of anterior and posterior lens surfaces at their corresponding flattest meridian as a function of age. Data was obtained from fits to biconic surfaces. Open circles represent data of the anterior lens surface and solid circles represent data of the posterior lens surface.

Figure 4.4 (a) Conic constants of anterior and posterior lens surfaces at their corresponding steepest meridian as a function of age. (b) Conic constants of the posterior lens surfaces at their corresponding flattest meridian as a function of age. Data was obtained from fits to biconic surfaces. Open triangles represent data from the anterior lens surface and solid triangles represent data from the posterior lens surface. 124

Figure 4.5 (a) Magnitude of lens surface astigmatism of the anterior and posterior lens surfaces as a function of age. (b) Correlation of the magnitude of astigmatism between anterior and posterior surfaces

Figure 4.6 (a) Anterior astigmatism power vector polar plot with the steepest meridian aligned at $90 \mathrm{deg}$, by convention; (b) Posterior astigmatism power vector polar plot. The angle (from the vertical axis) represents the relative angle between the anterior and posterior astigmatic axis. Each arrow represents one lens, the length of the vectors represent the magnitude of the corresponding astigmatism in diopters..... Error! Bookmark not defined.

Figure 4.7 Relative angle between anterior and posterior lens surface astigmatic axis as a function of age (Slope: $-0.53 \mathrm{deg} / \mathrm{year} ; \mathrm{r}=0.305 ; \mathrm{p}=0.071$ ); 126

Figure 4.8 (a) Trefoil-v $Z_{3}^{-3}$ : Anterior vs. Posterior (Slope $=0.387, r=0.467, p=0.004$ ) (b) RMS $3^{\text {rd }}$ order terms: Anterior vs. Posterior (Slope $=0.019 ; \mathrm{r}=0.477, \mathrm{p}=0.003$; (c) RMS Coma: Anterior vs. Posterior (Slope $=0.387 ; \mathrm{r}=0.617, \mathrm{p}=0.0001)(\mathrm{d}) 4^{\text {th }}$ order RMS: Anterior vs. Posterior (Slope $=0.387 ; \mathrm{r}=0.423, \mathrm{p}=0.010$. . 127

Figure 4.9 (a) Lens elevation high order RMS terms; RMS coma: slope $=-0.087 \mu \mathrm{m} / \mathrm{year}, \mathrm{r}$ $=0.582$ and $\mathrm{p}=0.0001$ for anterior surface, slope $=-0.123 \mu \mathrm{m} / \mathrm{year}, \mathrm{r}=0.515$ and $\mathrm{p}=0.001$ for posterior surface; (b) RMS spherical: slope $=-0.175 \mu \mathrm{m} /$ year, $\mathrm{r}=0.439, \mathrm{p}=0.007$ for anterior surface only (c) 3rd order RMS: slope $=-0.083 \mu \mathrm{m} /$ year, $r=0.564$ and $\mathrm{p}=0.0001$ for anterior surface only 130 
Figure 4.10 Relative contributions of different Zernike terms to the overall surface elevation maps (in terms of $\mathrm{RMS}^{2}$ ) with an asterisk the terms that change statistically significantly with age.

\section{$\underline{\text { Chapter } 5}$}

Figure 5.1 (a) Example movement of 20/20 Snellen E-letter across the display to change the eye's fixation angle stimulus; Acquisition at two different foci (cornea and lens) (b) Vertical cross-sectional scans of a patient's cornea while fixating foveally, i.e. along the line of sight; (c) Vertical cross-sectional scans of a patient's cornea, while fixating eccentrically, i.e. pupilary axis aligned with the OCT optical axis; (d) Vertical cross-sectional scans of the IOL implanted in the patient. Typical image acquisitions are obtained with the alignment as in (c) and (d). The yellow line indicates the iris plane, used for merging.

Figure 5.2 Anterior and posterior corneal topographic maps from Pentacam (upper panel) and OCT (lower panel). OCT-based maps were used in the computer eye models. Data are for 6-mm pupil. 144

Figure 5.3 Measured (LRT, $1^{\text {st }}$ column) and Simulated (from OCT-based geometry/biometry, $2^{\text {nd }}-5^{\text {th }}$ columns) wave aberration maps in three pseudophakic eyes. Simulated aberrations include wave aberration maps for the anterior cornea alone $\left(2^{\text {nd }}\right.$ column); anterior and posterior cornea ( $3^{\text {rd }}$ column); eye wave aberration with IOL assuming no tilt and decentration ( $4^{\text {th }}$ column); eye wave aberration with IOL with no tilt/decentration ( $5^{\text {th }}$ column). (a) Wave aberration maps including astigmatism (no tilt or defocus); (b) Wave aberrations for 3rd and higher order; Data are for 5-mm pupil, and for foveal fixation, i.e.including eye rotation. 146

Figure 5.4 (a) RMS Astigmatism; (b) RMS Spherical Aberration; (c) RMS Trefoil; (d) RMS Coma; (e) RMS Tetrafoil, for anterior corneal RMS (blue bars), total corneal RMS (red bars), simulated total eye RMS assuming centered IOL (green bars), simulated total eye RMS assuming real IOL tilt/decentration (purple bars); and experimental (LRT) total eye aberration (light blue bars) 147

Figure 5.5 Linear correlations between measured and simulated Zernike coefficients (astigmatism and 3rd and higher orders) in (a) S\#1 OD; (b) S\#2 OS; (c) S\#3 OS; (d) combining data from the three subjects (72 points). Data are for 5-mm pupil diameters.. 148

Figure 5.6 (a) MTFs (radial profiles) for astigmatism and higher order terms (b) MTFs (radial profiles) for 3rd and higher order aberrations, for LRT (blue lines), IOL with no tilt/decentration (green lines) and IOL with real tilt/decentration (purple lines). Data are for the three subjects, for 5-mm pupils.

Figure 5.7 (a) Corresponding Visual Strehl with Astigmatism and $3^{\text {rd }}$ and higher order aberration (b) Corresponding Visual Strehl with $3^{\text {rd }}$ and higher order aberration without Astigmatism (green bars present Simulation with IOL centered, purple bars present simulation with IOL tilt and decentration, blue bars present LRT). 150 


\section{Chapter 6}

Figure 6.1 Illustration of estimation of the optical IOL position. (1) Custom model eye is created with individual anatomical information; (2) Wave aberrations are computed for different IOL decentrations $1 \mathrm{~mm} \times 1 \mathrm{~mm}$ grid $(0.2 \mathrm{~mm}$ steps); 8 sample wave aberrations are shown; (3) Visual Strehl is calculated for every wave aberration (for 3-mm pupil diameter) and a VS pupillary map is created;(4) Location producing the maximum VS is identified. 163

Figure 6.2 the Visual Strehl (VS) pupillary maps for one eye (E\#1 OD), including: astigmatism and high order aberrations (in the first row) and only high order aberration (in the second row) under four conditions (without eye rotation (ER) and IOL tilt (IT) in the first column; with ER and without IT in the second column; without ER but with IT in third column; with ER and IT in the fourth column)....

Figure 6.3 the Visual Strehl (VS) pupillary maps for one eye (E\#1 OD) using anterior and posterior elevation maps (fitted to $6^{\text {th }}$ order polynomials), including: astigmatism and high order aberrations (in the first row) and only high order aberration (in the second row) under the same conditions in Figure 6.2.

Figure 6.4 Visual Strehl (VS) of E\#1 OD, E\#2 OS and E\#3 OS including Astigmatism and high order aberrations (HO) in the first row and including HOA only in the second row, with real ER and real IT.

Figure 6.5 (a) the maximum VS achieved by optimally placing the IOL, assuming aspheric corneas (blue lines) or real corneal elevation maps (red lines), in comparison with a perfect centered IOL, for subject E\#1). Solid lines/solid symbols represent the max VS, while dashed lines/opened symbols represent the VS of centered IOL in the pupil center. Thicker lines/ symbols represent VS including astigmatism and HOA, while thinner lines/smaller symbols represent VS including HOA only. (b) Optimal IOL position (max VS) using aspherical and real corneal elevation maps with four conditions. Square, diamond, circle and triangle symbols represent the condition (without ER and IT) in the first column; with real ER and without IT in the second column; without ER but with real IT in third column; with real ER and real IT in the fourth column) respectively. Star symbol represent the real IOL position in E\#1. The color codes are the same in (a). 168

Figure 6.6 (a) the Maximum Visual Strehl (by optimizing IOL decentration), the Visual Strehl with the real IOL decentration, and assuming a centered IOL, obtained from patient specific eye models in all three patients, with their actual anterior and posterior corneal topographies and real eye rotation and IOL tilt. Data shown include Astigmatism and HOA (solid bars) and HOA only (shaded bars). (b) The optimal IOL position (squares) and real IOL positions (circles), from estimations using astigmatism and HOA (closed) and HOA only (empty) for all three subjects. The stander deviations of real IOL positions are from measurements.

Figure 6.7 (a) RMS astigmatism; (b) RMS Coma and (c) RMS Trefoil changing with four conditions (no ER, no IT; Real ER, no IT; No ER, Real IT; Real ER and Real IT). Double solid line, single solid line and dashed line represent the max VS including astigmatism \& $\mathrm{HO}$, the max VS including HOA only, and the VS of a centered IOL in the pupil center. The color codes are the same in Figure 6.4. 170

Figure 6.8 (a) RMS astigmatism; (b) RMS Coma and (c) RMS Trefoil with real ER and IT. Dark solid bars represent the optimal IOL position of max VS including astigmatism \& HOA, shade bars represent the optimal IOL position of max VS including HOA only, light solid bars represent the real IOL position in the three subjects, and lighter solid bars represent the 
IOL position centered in the pupil center. The three eyes are coded as the same in Figure 6.5.

Figure 6.9 Visual Strehl (VS) of high order aberration through focus (from 3rd order Zernike terms with best focus) (in the first row) and without defocus (in second row) as a function of IOL position. (a) E1; (b) E2; (c) E3; 172

Figure 6.10 the residual refractive error (the first row) and astigmatism power (the second row) as a function of IOL positions of E\#1 OD (the first column), E\#2 OS (the second column) and E\#3 OS (the third column). (in D) 


\section{TABLE LIST}

Table 1.1 Summary of the eye-models, number of surfaces in each model and average population data used in each model (MERINO 2015).

Table 1.2 Summary of Pseudophakic eye model (MERINO 2015)

Table 3.1 Radii of curvature, axial biometry, and refractive indices, in the physical model eye, and the five patient eyes

Table 3.2 A-G coefficients in Eq.2.4for the physical model eye in air and water and the 5 eyes of the study

Table 4.1 Mean and standard deviation of the RMS of high-order Zernike coefficients for anterior and posterior lens surfaces. The Pearson correlation coefficient and the p-value are shown for the correlation with age of these parameters in each surface and between surfaces.

Table 5.2 Individual OCT-based biometrical and geometrical data used in the computer model eyes. Data are shown as average \pm standard deviation of repeated measurements.

Table 5.3 Eye rotation and IOL tilt and decentration data from OCT-based Purkinje-like methods. Data are average \pm standard deviation of 5 repeated measurements. Rotation/tilt $\mathrm{X}$ stands for rotation/tilt around the horizontal axis; Rotation/tilt Y stands for rotation/tilt around the vertical axis. Decentration $\mathrm{X} / \mathrm{Y}$ stands for horizontal /vertical decentration. 145

Table 5.4 Anterior and posterior radius of curvature (R), asphericity $(\mathrm{Q})$ from profilometry and OCT, and the IOL power estimated from profilometry and OCT. OCT-based data were used in the computer eye models.

Table 6.1 show measurements of eye rotation, IOL tilt and decentration (horizontal and vertical components) for each eye. 


\section{BIBLIOGRAPHY}

A

A. De La Hoz, E. M.-E., H. Subhash, D. Pascual, N. Bekesi, C. Dorronsoro, N. Alejandre-Alba, S. Marcos. (2016). "Design and evaluation of a surface-changing accommodating IOL." IOVS 58(ARVO E-Abstract 3131 (2016)).

Accardo, P. A. and S. Pensiero (2002). "Neural network-based system for early keratoconus detection from corneal topography." J Biomed Inform 35(3): 151-159.

Adhi, M., D. Ferrara, R. F. Mullins, C. R. Baumal, K. J. Mohler, M. F. Kraus, J. Liu, E. Badaro, T. Alasil, J. Hornegger, J. G. Fujimoto, J. S. Duker and N. K. Waheed (2015). "Characterization of Choroidal Layers in Normal Aging Eyes Using Enface Swept-Source Optical Coherence Tomography." PLoS One 10(7): e0133080.

Ale, J. B. (2011). "Intraocular lens tilt and decentration: a concern for contemporary IOL designs." Nepal J Ophthalmol 3(1): 68-77.

Alio, J. L., B. Elkady, D. Ortiz and G. Bernabeu (2008). "Microincision multifocal intraocular lens with and without a capsular tension ring: optical quality and clinical outcomes." J Cataract Refract Surg 34(9): 1468-1475.

Alio, J. L., A. B. Plaza-Puche, J. Javaloy, M. J. Ayala and A. Vega-Estrada (2013). "Clinical and optical intraocular performance of rotationally asymmetric multifocal IOL plate-haptic design versus C-loop haptic design." J Refract Surg 29(4): 252-259.

Altmann, G. E., L. D. Nichamin, S. S. Lane and J. S. Pepose (2005). "Optical performance of 3 intraocular lens designs in the presence of decentration." J Cataract Refract Surg 31(3): 574-585.

Amano, S., Y. Amano, S. Yamagami, T. Miyai, K. Miyata, T. Samejima and T. Oshika (2004). "Age-related changes in corneal and ocular higher-order wavefront aberrations." Am J Ophthalmol 137(6): 988-992.

Applegate, R. A., Ballentine, C., Gross, H., Sarver, E. J., Sarver, C. A. (2003). "Visual acuity as a function of Zernike mode and level of root mean square error." Optometry and Vision Science 80(2)(97-105).

Applegate, R. A., G. Hilmantel, H. C. Howland, E. Y. Tu, T. Starck and E. J. Zayac (2000). "Corneal first surface optical aberrations and visual performance." J Refract Surg 16(5): 507-514.

Applegate, R. A., J. D. Marsack, R. Ramos and E. J. Sarver (2003). "Interaction between aberrations to improve or reduce visual performance." J Cataract Refract Surg 29(8): 1487-1495.

Applegate, R. A., J. D. Marsack and L. N. Thibos (2006). "Metrics of retinal image quality predict visual performance in eyes with 20/17 or better visual acuity." Optom Vis Sci 83(9): 635-640.

Arnulf, A., Dupuy, O. (1956). "Méthode objective pour l'etude des défauts du systéme optique de l'oeil " Problems in contemporary optics (pp. 330-335):(Inst. Naz. Ottica, Arcetri-Firenze.).

Artal, P., A. Guirao, E. Berrio and D. R. Williams (2001). "Compensation of corneal aberrations by the internal optics in the human eye." J Vis 1(1): 1-8.

Astbury, N. and B. Ramamurthy (2006). "How to avoid mistakes in biometry." Community Eye Health 19(60): 70-71. 
Atchison, D. A. (1989). "Optical design of intraocular lenses. I. On-axis performance." Optom Vis Sci 66(8): 492-506.

Atchison, D. A. (1989). "Optical design of intraocular lenses. III. On-axis performance in the presence of lens displacement." Optom Vis Sci 66(10): 671-681.

Atchison, D. A. (1991). "Design of aspheric intraocular lenses." Ophthalmic Physiol Opt 11(2): 137-146.

Atchison, D. A. (1995). "Accommodation and presbyopia." Ophthalmic Physiol Opt 15(4): 255272.

Atchison, D. A., E. L. Markwell, S. Kasthurirangan, J. M. Pope, G. Smith and P. G. Swann (2008). "Age-related changes in optical and biometric characteristics of emmetropic eyes." J Vis 8(4): 29 21-20.

Atchison DA, S. G. (2000). "Optics of the Human Eye." Edinburgh, UK Butterworth-Heinemann.

Atchison, D. A. and G. Smith (1995). "Continuous gradient index and shell models of the human lens." Vision Res 35(18): 2529-2538.

Atchison, D. A. and G. Smith (2004). "Possible errors in determining axial length changes during accommodation with the IOLMaster." Optom Vis Sci 81(4): 283-286.

Auffarth, G. U., M. Wilcox, J. C. Sims, C. McCabe, T. A. Wesendahl and D. J. Apple (1995). "Analysis of 100 explanted one-piece and three-piece silicone intraocular lenses." Ophthalmology 102(8): 1144-1150.

Augusteyn, R. C., A. M. Rosen, D. Borja, N. M. Ziebarth and J. M. Parel (2006). "Biometry of primate lenses during immersion in preservation media." Mol Vis 12: 740-747.

Auran, J. D., C. J. Koester and A. Donn (1990). "In vivo measurement of posterior chamber intraocular lens decentration and tilt." Arch Ophthalmol 108(1): 75-79.

\section{B}

B, A. N. a. R. (2006). "How to avoid mistakes in biometry." Community Eye Health 19((60)): 70 71.

Baikoff, G. (2006). "Anterior segment OCT and phakic intraocular lenses: a perspective." $\underline{\mathrm{J} \text { Cataract }}$ Refract Surg 32(11): 1827-1835.

Barbero, S. (2006). "Refractive power of a multilayer rotationally symmetric model of the human cornea and tear film." J Opt Soc Am A Opt Image Sci Vis 23(7): 1578-1585.

Barbero, S., S. Marcos and J. Merayo-Lloves (2002). "Corneal and total optical aberrations in a unilateral aphakic patient." J Cataract Refract Surg 28(9): 1594-1600.

Barbero, S., S. Marcos, J. Montejo and C. Dorronsoro (2011). "Design of isoplanatic aspheric monofocal intraocular lenses." Opt Express 19(7): 6215-6230.

Barer, R., K. F. Ross and S. Tkaczyk (1953). "Refractometry of living cells." Nature 171(4356): 720-724.

Barry, J. C., M. Dunne and T. Kirschkamp (2001). "Phakometric measurement of ocular surface radius of curvature and alignment: evaluation of method with physical model eyes." Ophthalmic Physiol Opt 21(6): 450-460.

Baumeister, M., B. Neidhardt, J. Strobel and T. Kohnen (2005). "Tilt and decentration of threepiece foldable high-refractive silicone and hydrophobic acrylic intraocular lenses with 6-mm optics in an intraindividual comparison." Am J Ophthalmol 140(6): 1051-1058. 
Bedford, R. E. and G. Wyszecki (1957). "Axial chromatic aberration of the human eye." J Opt Soc Am 47(6): 564-565.

Beebe, D. C. T. 1. i. P. L. K., A. Alm (Eds.) (2003). " Adler's Physiology of the Eye." ed 10. Inc, St Louis, (MO, Mosby): 117-158.

Beers, A. P. and G. L. Van Der Heijde (1994). "In vivo determination of the biomechanical properties of the component elements of the accommodation mechanism." Vision Res 34(21): 28972905.

Bellini, N., M. J. Cox, D. J. Harper, S. R. Stott, P. C. Ashok, K. Dholakia, S. Kawaguchi, R. King, T. Horton and C. T. Brown (2014). "The application of optical coherence tomography to image subsurface tissue structure of Antarctic krill Euphausia superba." PLoS One 9(10): e110367.

Benedek, G. B. (1971). "Theory of transparency of the eye." Appl Opt 10(3): 459-473.

Bezerra, H. G., M. A. Costa, G. Guagliumi, A. M. Rollins and D. I. Simon (2009). "Intracoronary optical coherence tomography: a comprehensive review clinical and research applications." JACC Cardiovasc Interv 2(11): 1035-1046.

Birkenfeld, J., A. de Castro and S. Marcos (2014). "Contribution of shape and gradient refractive index to the spherical aberration of isolated human lenses." Invest Ophthalmol Vis Sci 55(4): 25992607.

Bolz, M., A. Prinz, W. Drexler and O. Findl (2007). "Linear relationship of refractive and biometric lenticular changes during accommodation in emmetropic and myopic eyes." Br J Ophthalmol 91(3): 360-365.

Borja, D., F. Manns, A. Ho, N. Ziebarth, A. M. Rosen, R. Jain, A. Amelinckx, E. Arrieta, R. C. Augusteyn and J. M. Parel (2008). "Optical power of the isolated human crystalline lens." Invest Ophthalmol Vis Sci 49(6): 2541-2548.

Borja, D., D. Siedlecki, A. de Castro, S. Uhlhorn, S. Ortiz, E. Arrieta, J. M. Parel, S. Marcos and F. Manns (2010). "Distortions of the posterior surface in optical coherence tomography images of the isolated crystalline lens: effect of the lens index gradient." Biomed Opt Express 1(5): 1331-1340.

Born, M., \& Wolf, E. (1993). "Principles of Optics." Oxford, U.K. Pergamon Press(6th ed.).

Born M. , W. E. (1999). "Principles of Optics." Cambridge University Press 7th Edition( ISBN 978$0-521-64222-4)$.

Bradley, A. (1992). "Glenn A. Fry Award Lecture 1991: perceptual manifestations of imperfect optics in the human eye: attempts to correct for ocular chromatic aberration." Optom Vis Sci 69(7): 515-521.

Bradley. A and L. N. Thibos. (1995). "Modeling off-axis vision - I: The optical effects of decentering visual targets or the eye's entrance pupil." In E Peli, editor Vision models for target detection and resolution(World Scientific Press): pages 313-337.

Brito, P., J. Salgado-Borges, H. Neves, J. Gonzalez-Meijome and M. Monteiro (2015). "Lightdistortion analysis as a possible indicator of visual quality after refractive lens exchange with diffractive multifocal intraocular lenses." J Cataract Refract Surg 41(3): 613-622.

Bron, A. J., G. F. Vrensen, J. Koretz, G. Maraini and J. J. Harding (2000). "The ageing lens." Ophthalmologica 214(1): 86-104.

Brooks, A. M., R. H. West and W. E. Gillies (1986). "The risks of precipitating acute angle-closure glaucoma with the clinical use of mydriatic agents." Med J Aust 145(1): 34-36.

Brown, N. (1972). "An advanced slit-image camera." Br J Ophthalmol 56(8): 624-631.

Bueeler, M., M. Mrochen and T. Seiler (2003). "Maximum permissible lateral decentration in aberration-sensing and wavefront-guided corneal ablation." J Cataract Refract Surg 29(2): 257-263. 
Buhren, J., K. Pesudovs, T. Martin, A. Strenger, G. Yoon and T. Kohnen (2009). "Comparison of optical quality metrics to predict subjective quality of vision after laser in situ keratomileusis." $\underline{\mathbf{J}}$ Cataract Refract Surg 35(5): 846-855.

Burd, H. J., S. J. Judge and M. J. Flavell (1999). "Mechanics of accommodation of the human eye." Vision Res 39(9): 1591-1595.

C

Cagini, C., F. Pietrolucci, M. Lupidi, M. Messina, F. Piccinelli and T. Fiore (2015). "Influence of pseudophakic lens capsule opacification on spectral domain and time domain optical coherence tomography image quality." Curr Eye Res 40(6): 579-584.

Campbell, C. E. (2010). "Nested shell optical model of the lens of the human eye." J Opt Soc Am A Opt Image Sci Vis 27(11): 2432-2441.

Campbell, F. W. and R. W. Gubisch (1966). "Optical quality of the human eye." J Physiol 186(3): 558-578.

Campbell, M. C. (1984). "Measurement of refractive index in an intact crystalline lens." Vision Res 24(5): 409-415.

Carlson, A. N., W. C. Stewart and P. C. Tso (1998). "Intraocular lens complications requiring removal or exchange." Surv Ophthalmol 42(5): 417-440.

Carney, L. G., J. C. Mainstone and B. A. Henderson (1997). "Corneal topography and myopia. A cross-sectional study." Invest Ophthalmol Vis Sci 38(2): 311-320.

Castejon-Mochon, J. F., N. Lopez-Gil, A. Benito and P. Artal (2002). "Ocular wave-front aberration statistics in a normal young population." Vision Res 42(13): 1611-1617.

Chang, D. H. and G. O. t. Waring (2014). "The subject-fixated coaxially sighted corneal light reflex: a clinical marker for centration of refractive treatments and devices." Am J Ophthalmol 158(5): 863874.

Charman, W. N. (2008). "The eye in focus: accommodation and presbyopia." Clin Exp Optom 91(3): 207-225.

Chen, Q., T. Leng, L. L. Zheng, L. Kutzscher, L. De Sisternes and D. L. Rubin (2014). "An improved optical coherence tomography-derived fundus projection image for drusen visualization." Retina 34(5): 996-1005.

Cheng, J., L. Duan, D. W. Wong, D. Tao, M. Akiba and J. Liu (2014). "Speckle reduction in optical coherence tomography by image registration and matrix completion." Med Image Comput Comput Assist Interv 17(Pt 1): 162-169.

Cheng, X., L. N. Thibos and A. Bradley (2003). "Estimating visual quality from wavefront aberration measurements." J Refract Surg 19(5): S579-584.

Chinn, S. R., E. A. Swanson and J. G. Fujimoto (1997). "Optical coherence tomography using a frequency-tunable optical source." Opt Lett 22(5): 340-342.

Cholkar, K., H. M. Trinh, A. D. Vadlapudi and A. K. Mitra (2014). "Synthesis and Characterization of Ganciclovir Long Chain Lipid Prodrugs." Adv Ophthalmol Vis Syst 1(2).

Choma, M., M. Sarunic, C. Yang and J. Izatt (2003). "Sensitivity advantage of swept source and Fourier domain optical coherence tomography." Opt Express 11(18): 2183-2189.

Choma, M. A., Hsu, K., Izatt, J.A. (2005). "Swept source optical coherence tomography using an all-fiber 1300-nm ring laser source." J. Biomed. Opt., 10.

Chopdar, A. A., T. (2014). "Multimodal retinal imaging." JP Medical Ltd. 
Coleman, D. J., R. L. Jack, L. A. Franzen and S. C. Werner (1972). "High resolution B-scan ultrasonography of the orbit. V. Eye changes of Graves' disease." Arch Ophthalmol 88(5): 465-471.

Collins, M. J., C. F. Wildsoet and D. A. Atchison (1995). "Monochromatic aberrations and myopia." Vision Res 35(9): 1157-1163.

Cook, C. A., J. F. Koretz, A. Pfahnl, J. Hyun and P. L. Kaufman (1994). "Aging of the human crystalline lens and anterior segment." Vision Res 34(22): 2945-2954.

Crnej, A., N. Hirnschall, Y. Nishi, V. Gangwani, J. Tabernero, P. Artal and O. Findl (2011). "Impact of intraocular lens haptic design and orientation on decentration and tilt." J Cataract Refract Surg 37(10): 1768-1774.

Croft, M. A., A. Glasser and P. L. Kaufman (2001). "Accommodation and presbyopia." Int Ophthalmol Clin 41(2): 33-46.

Croft, M. A., J. P. McDonald, N. V. Nadkarni, T. L. Lin and P. L. Kaufman (2009). "Age-related changes in centripetal ciliary body movement relative to centripetal lens movement in monkeys." Exp Eye Res 89(6): 824-832.

\section{D}

David A Atchison, G. S. (2000). "Optics of the human eye." utterworthHeinemann, Oxford.

David Borja, F. M., Arthur Ho, Noel Ziebarth, Alexandre M Rosen, Rakhi Jain, Adriana Amelinckx, Esdras Arrieta, Robert C Augusteyn, and Jean Marie Parel. (2008). "Optical power of the isolated human crystalline lens. ." Invest Ophthalmol Vis Sci 49(6)(2541-2548).

de Boer, J. F., B. Cense, B. H. Park, M. C. Pierce, G. J. Tearney and B. E. Bouma (2003). "Improved signal-to-noise ratio in spectral-domain compared with time-domain optical coherence tomography." Opt Lett 28(21): 2067-2069.

de Castro, A., J. Birkenfeld, B. Maceo, F. Manns, E. Arrieta, J. M. Parel and S. Marcos (2013). "Influence of shape and gradient refractive index in the accommodative changes of spherical aberration in nonhuman primate crystalline lenses." Invest Ophthalmol Vis Sci 54(9): 6197-6207.

de Castro, A., S. Ortiz, E. Gambra, D. Siedlecki and S. Marcos (2010). "Three-dimensional reconstruction of the crystalline lens gradient index distribution from OCT imaging." Opt Express 18(21): 21905-21917.

de Castro, A., P. Rosales and S. Marcos (2007). "Tilt and decentration of intraocular lenses in vivo from Purkinje and Scheimpflug imaging. Validation study." J Cataract Refract Surg 33(3): 418-429.

de Castro, A., D. Siedlecki, D. Borja, S. Uhlhorn, J. M. Parel, F. Manns and S. Marcos (2011). "Age-dependent variation of the Gradient Index profile in human crystalline lenses." J Mod Opt 58(19-20): 1781-1787.

Demer, J. L., R. A. Clark, B. T. Crane, J. R. Tian, A. Narasimhan and S. Karim (2008). "Functional anatomy of the extraocular muscles during vergence." Prog Brain Res 171: 21-28.

Deshpande, V. (1999). "Indian influences on early Chinese ophthalmology: glaucoma as a case study." Bulletin of the School of Oriental and African Studies 62.2(1999): 306-322.

Deshpande, V. (2000). "Ophthalmic surgery: a chapter in the history of Sino-Indian medical contacts." Bulletin of the School of Oriental and African Studies Studies63.3(2000): 370-388. .

Dorronsoro, C., S. Barbero, L. Llorente and S. Marcos (2003). "On-eye measurement of optical performance of rigid gas permeable contact lenses based on ocular and corneal aberrometry." $\underline{\text { Optom }}$ Vis Sci 80(2): 115-125.

Douthwaite, W. A. (2002). "Application of linear regression to videokeratoscope data for tilted surfaces." Ophthalmic Physiol Opt 22(1): 46-54. 
Drexler, W., O. Findl, R. Menapace, G. Rainer, C. Vass, C. K. Hitzenberger and A. F. Fercher (1998). "Partial coherence interferometry: a novel approach to biometry in cataract surgery." Am J Ophthalmol 126(4): 524-534.

Dubbelman, M., V. A. Sicam and R. G. van der Heijde (2007). "The contribution of the posterior surface to the coma aberration of the human cornea." J Vis 7(7): 10 11-18.

Dubbelman, M. and G. L. Van der Heijde (2001). "The shape of the aging human lens: curvature, equivalent refractive index and the lens paradox." Vision Res 41(14): 1867-1877.

Dubbelman, M., G. L. van der Heijde and H. A. Weeber (2001). "The thickness of the aging human lens obtained from corrected Scheimpflug images." Optom Vis Sci 78(6): 411-416.

Dubbelman, M., G. L. Van der Heijde and H. A. Weeber (2005). "Change in shape of the aging human crystalline lens with accommodation." Vision Res 45(1): 117-132.

Dubbelman, M., G. L. Van der Heijde, H. A. Weeber and G. F. Vrensen (2003). "Changes in the internal structure of the human crystalline lens with age and accommodation." Vision Res 43(22): 2363-2375.

Dubbelman, M., R. G. van der Heijde and H. A. Weeber (2005). "Comment on "Scheimpflug and high-resolution magnetic resonance imaging of the anterior segment: a comparative study"." J Opt Soc Am A Opt Image Sci Vis 22(6): 1216-1218; discussion 1219-1220.

Dubbelman, M., H. A. Weeber, R. G. van der Heijde and H. J. Volker-Dieben (2002). "Radius and asphericity of the posterior corneal surface determined by corrected Scheimpflug photography." Acta Ophthalmol Scand 80(4): 379-383.

Dunne, M. C., L. N. Davies, E. A. Mallen, T. Kirschkamp and J. C. Barry (2005). "Non-invasive phakometric measurement of corneal and crystalline lens alignment in human eyes." Ophthalmic Physiol Opt 25(2): 143-152.

Dunne, M. C., G. P. Misson, E. K. White and D. A. Barnes (1993). "Peripheral astigmatic asymmetry and angle alpha." Ophthalmic Physiol Opt 13(3): 303-305.

Dunne, M. C., J. M. Royston and D. A. Barnes (1992). "Normal variations of the posterior corneal surface." Acta Ophthalmol (Copenh) 70(2): 255-261.

Dupps, W. J., Jr. and S. E. Wilson (2006). "Biomechanics and wound healing in the cornea." Exp Eye Res 83(4): 709-720.

\section{$\mathbf{E}$}

Elsheikh, A., D. Alhasso and P. Rama (2008). "Biomechanical properties of human and porcine corneas." Exp Eye Res 86(5): 783-790.

Enrique J. Fern’andez, A. U., Pedro M. Prieto, Boris Hermann, Wolfgang Drexler, and Pablo Artal (2005). "Ocular aberrations as a function of wavelength in the near infrared measured with a femtosecond laser." Opt Express 13(2)(400-49,).

Eppig, T., K. Scholz, A. Loffler, A. Messner and A. Langenbucher (2009). "Effect of decentration and tilt on the image quality of aspheric intraocular lens designs in a model eye." $\underline{\mathrm{J} \text { Cataract Refract }}$ Surg 35(6): 1091-1100.

Era. (2008). "Cataract Surgery in the Modern." American Academy of Ophthalmology.(http://www.aaofoundation.org/what/heritage/exhibits/online/cataract/modern.cfm ). 
Fedorov SN, K. A., Kolinko AI. (1967). "Estimation of optical power of the intraocular lens." Vestn Oftalmol 80: 27-31.

Fercher, A. F., C. K. Hitzenberger, W. Drexler, G. Kamp and H. Sattmann (1993). "In vivo optical coherence tomography." Am J Ophthalmol 116(1): 113-114.

Fercher, A. F., Hitzenberger, C.K., Kamp, G., Elzaiat, S.Y. (1995). "Measurement of intraocular distances by backscattering spectral interferometry." Opt. Commun. 117(43-48).

Fercher, A. F., K. Mengedoht and W. Werner (1988). "Eye-length measurement by interferometry with partially coherent light." Opt Lett 13(3): 186-188.

Findl, O. (2005). "Biometry and intraocular lens power calculation." Curr Opin Ophthalmol 16(1): 61-64.

Findl, O., K. Kriechbaum, S. Sacu, B. Kiss, K. Polak, J. Nepp, G. Schild, G. Rainer, S. Maca, V. Petternel, B. Lackner and W. Drexler (2003). "Influence of operator experience on the performance of ultrasound biometry compared to optical biometry before cataract surgery." J Cataract Refract Surg 29(10): 1950-1955.

Fink, W. and D. Micol (2006). "simEye: Computer-based simulation of visual perception under various eye defects using Zernike polynomials." J Biomed Opt 11(5): 054011.

\section{G}

Gambra, E., S. Ortiz, P. Perez-Merino, M. Gora, M. Wojtkowski and S. Marcos (2013). "Static and dynamic crystalline lens accommodation evaluated using quantitative 3-D OCT." Biomed Opt Express 4(9): 1595-1609.

Garner, L. F. (1983). "Mechanisms of accommodation and refractive error." Ophthalmic Physiol Opt 3(3): 287-293.

Garner, L. F. and M. K. Yap (1997). "Changes in ocular dimensions and refraction with accommodation." Ophthalmic Physiol Opt 17(1): 12-17.

Gatinel, D. and T. Hoang-Xuan (2007). "Objective assessment of the quality of vision before and after repositioning of a dislocated iris-fixated aphakic anterior chamber lens." J Refract Surg 23(9 Suppl): S1005-1010.

Glasser, A. (2008). "Restoration of accommodation: surgical options for correction of presbyopia." Clin Exp Optom 91(3): 279-295.

Glasser, A. and M. C. Campbell (1998). "Presbyopia and the optical changes in the human crystalline lens with age." Vision Res 38(2): 209-229.

Glasser, A. and M. C. Campbell (1999). "Biometric, optical and physical changes in the isolated human crystalline lens with age in relation to presbyopia." Vision Res 39(11): 1991-2015.

Glasser, A., M. A. Croft and P. L. Kaufman (2001). "Aging of the human crystalline lens and presbyopia." Int Ophthalmol Clin 41(2): 1-15.

Golubovic, B., B. E. Bouma, G. J. Tearney and J. G. Fujimoto (1997). "Optical frequency-domain reflectometry using rapid wavelength tuning of a Cr4+:forsterite laser." Opt Lett 22(22): 1704-1706.

Goncharov, A. V. and C. Dainty (2007). "Wide-field schematic eye models with gradient-index lens." J Opt Soc Am A Opt Image Sci Vis 24(8): 2157-2174.

Goss, D. A., H. G. Van Veen, B. B. Rainey and B. Feng (1997). "Ocular components measured by keratometry, phakometry, and ultrasonography in emmetropic and myopic optometry students." Optom Vis Sci 74(7): 489-495. 
Grulkowski, I., M. Gora, M. Szkulmowski, I. Gorczynska, D. Szlag, S. Marcos, A. Kowalczyk and M. Wojtkowski (2009). "Anterior segment imaging with Spectral OCT system using a high-speed CMOS camera." Opt Express 17(6): 4842-4858.

Guillon, M., D. P. Lydon and C. Wilson (1986). "Corneal topography: a clinical model." Ophthalmic Physiol Opt 6(1): 47-56.

Guirao, A. and P. Artal (2000). "Corneal wave aberration from videokeratography: accuracy and limitations of the procedure." J Opt Soc Am A Opt Image Sci Vis 17(6): 955-965.

Guirao, A. and D. R. Williams (2003). "A method to predict refractive errors from wave aberration data." Optom Vis Sci 80(1): 36-42.

Gullstrand, A. (1909). "Appendix II in: H. von Helmholtz "'Handbuch der Physiologischen Optik' 3rd edition(part I).

Gustafsson, J., E. Terenius, J. Buchheister and P. Unsbo (2001). "Peripheral astigmatism in emmetropic eyes." Ophthalmic Physiol Opt 21(5): 393-400.

Guyton, D. L., H. Uozato and H. J. Wisnicki (1990). "Rapid determination of intraocular lens tilt and decentration through the undilated pupil." Ophthalmology 97(10): 1259-1264.

\section{H}

Ha Usler, G. and M. W. Lindner (1998). ""Coherence radar" and "spectral radar"-new tools for dermatological diagnosis." J Biomed Opt 3(1): 21-31.

Haigis W. (2004). "The Haigis formula." In: Shammas HJ, ed, Intraocular Lens Power Calculations. Thorofare, NJ, Slack, : 41-57.

Hartmann, J. (1900). "Bemerkungen über den Bau und die Justierung von Spektrgraphen." $\underline{Z}$ Instumentenkd, 20(47-58).

Hartmann, J. (1904). "Objektivuntersuchungen." Z Instrumentenkd 24(1-32).

Hashemi, H., M. KhabazKhoob, K. Yazdani, S. Mehravaran, E. Jafarzadehpur and A. Fotouhi (2010). "Distribution of angle kappa measurements with Orbscan II in a population-based survey." J Refract Surg 26(12): 966-971.

Hayashi, H., K. Hayashi, F. Nakao and F. Hayashi (1998). "Area reduction in the anterior capsule opening in eyes of diabetes mellitus patients." J Cataract Refract Surg 24(8): 1105-1110.

Hayashi, K. and H. Hayashi (2006). "Long-term changes in corneal surface configuration after penetrating keratoplasty." Am J Ophthalmol 141(2): 241-247.

Hayashi, K., H. Hayashi, K. Matsuo, F. Nakao and F. Hayashi (1998). "Anterior capsule contraction and intraocular lens dislocation after implant surgery in eyes with retinitis pigmentosa." Ophthalmology 105(7): 1239-1243.

Hayashi, K., H. Hayashi, F. Nakao and F. Hayashi (1998). "Comparison of decentration and tilt between one piece and three piece polymethyl methacrylate intraocular lenses." Br J Ophthalmol 82(4): 419-422.

Hayashi, K., H. Hayashi, F. Nakao and F. Hayashi (1999). "Intraocular lens tilt and decentration after implantation in eyes with glaucoma." J Cataract Refract Surg 25(11): 1515-1520.

He, J. C., S. A. Burns and S. Marcos (2000). "Monochromatic aberrations in the accommodated human eye." Vision Res 40(1): 41-48.

He, L., R. Ren, H. Yang, C. Hardin, L. Reyes, J. Reynaud, S. K. Gardiner, B. Fortune, S. Demirel and C. F. Burgoyne (2014). "Anatomic vs. acquired image frame discordance in spectral domain optical coherence tomography minimum rim measurements." PLoS One 9(3): e92225. 
Hemenger, R. P., L. F. Garner and C. S. Ooi (1995). "Change with age of the refractive index gradient of the human ocular lens." Invest Ophthalmol Vis Sci 36(3): 703-707.

Hermans, E. A., M. Dubbelman, G. L. van der Heijde and R. M. Heethaar (2006). "Estimating the external force acting on the human eye lens during accommodation by finite element modelling." Vision Res 46(21): 3642-3650.

Hermans, E. A., P. J. Pouwels, M. Dubbelman, J. P. Kuijer, R. G. van der Heijde and R. M. Heethaar (2009). "Constant volume of the human lens and decrease in surface area of the capsular bag during accommodation: an MRI and Scheimpflug study." Invest Ophthalmol Vis Sci 50(1): 281-289.

Hoffer, K. J. (1980). "Steps for IOL power calculation." J Am Intraocul Implant Soc 6(4): 370.

Hoffer, K. J. (1993). "The Hoffer Q formula: a comparison of theoretic and regression formulas." J Cataract Refract Surg 19(6): 700-712.

Holladay, J. T., D. Calogero, G. Hilmantel, A. Glasser, S. MacRae, S. Masket, W. Stark, M. E. Tarver, T. Nguyen and M. Eydelman (2017). "Special Report: American Academy of Ophthalmology Task Force Summary Statement for Measurement of Tilt, Decentration, and Chord Length." Ophthalmology 124(1): 144-146.

Holladay, J. T., P. A. Piers, G. Koranyi, M. van der Mooren and N. E. Norrby (2002). "A new intraocular lens design to reduce spherical aberration of pseudophakic eyes." J Refract Surg 18(6): 683-691.

Holladay, J. T., T. C. Prager, T. Y. Chandler, K. H. Musgrove, J. W. Lewis and R. S. Ruiz (1988). "A three-part system for refining intraocular lens power calculations." J Cataract Refract Surg 14(1): $17-24$.

Howarth, P. A. (1984). "The lateral chromatic aberration of the eye." Ophthalmic Physiol Opt 4(3): 223-226.

Howarth, P. A. and A. Bradley (1986). "The longitudinal chromatic aberration of the human eye, and its correction." Vision Res 26(2): 361-366.

Howland, B. (1960). "Use of crossed cylinder lens in photographic lens evaluation." Applied Optics 7(1587-1588).

Howland, B. and H. C. Howland (1976). "Subjective measurement of high-order aberrations of the eye." Science 193(4253): 580-582.

Howland, H. C. and B. Howland (1977). "A subjective method for the measurement of monochromatic aberrations of the eye." J Opt Soc Am 67(11): 1508-1518.

Huang, D., E. A. Swanson, C. P. Lin, J. S. Schuman, W. G. Stinson, W. Chang, M. R. Hee, T. Flotte, K. Gregory, C. A. Puliafito and et al. (1991). "Optical coherence tomography." Science 254(5035): 1178-1181.

Hurd, L. B., 2nd and M. L. Feldman (1994). "Purkinje-like cells in rat cochlear nucleus." $\underline{\text { Hear Res }}$ 72(1-2): 143-158.

\section{I}

Institute., A. N. S. (2004). "Methods for reporting optical aberrations of the eye, 2004., International Organization for Standardization." American National Standard for Ophthalmics ANSI Z80.28

Iskander, D. R. (2006). "Computational aspects of the visual Strehl ratio." Optom Vis Sci 83(1): 57 59 .

Izatt, J. A., M. R. Hee, E. A. Swanson, C. P. Lin, D. Huang, J. S. Schuman, C. A. Puliafito and J. G. Fujimoto (1994). "Micrometer-scale resolution imaging of the anterior eye in vivo with optical coherence tomography." Arch Ophthalmol 112(12): 1584-1589. 
James T. Schwiegerling (2013). "Eye Axes and Their Relevance to Alignment of Corneal Refractive Procedures." Journal of Refractive Surgery 29(8): 515-516.

John A. (2001). "The eye and orbit, Fielding." In: Cosgrove D, editor. Clinical Ultrasound((6)): 5995.

Jones, C. E., D. A. Atchison, R. Meder and J. M. Pope (2005). "Refractive index distribution and optical properties of the isolated human lens measured using magnetic resonance imaging (MRI)." Vision Res 45(18): 2352-2366.

Jones, C. E., D. A. Atchison and J. M. Pope (2007). "Changes in lens dimensions and refractive index with age and accommodation." Optom Vis Sci 84(10): 990-995.

Juan Tabernero, N. H., Sophie Tatzreiter, Oliver Findl and Pablo Artal (2012). "Determining The Stability Of Implanted Iols With A Dynamic Purkinje-meter System." Invest Ophthalmol Vis Sci 53(E-Abstract 1373.@ARVO).

Judge, S. J. and H. J. Burd (2002). "Modelling the mechanics of accommodation and presbyopia." Ophthalmic Physiol Opt 22(5): 397-400.

\section{$\mathbf{K}$}

Kamiya, K., K. Shimizu, M. Yamagishi, A. Igarashi and H. Kobashi (2015). "Anterior and Posterior Corneal Astigmatism after Refractive Lenticule Extraction for Myopic Astigmatism." J Ophthalmol 2015: 915853.

Kasthurirangan, S., E. L. Markwell, D. A. Atchison and J. M. Pope (2008). "In vivo study of changes in refractive index distribution in the human crystalline lens with age and accommodation." Invest Ophthalmol Vis Sci 49(6): 2531-2540.

Kasthurirangan, S., E. L. Markwell, D. A. Atchison and J. M. Pope (2011). "MRI study of the changes in crystalline lens shape with accommodation and aging in humans." J Vis 11(3).

Kasthurirangan, S., Markwell, E., Atchison, D.A., Pope, J.M., Smith, G. (2007). "Magnetic resonance imaging study of crystalline lens shape with accommodation and age. ." Invest. Ophtalmol. Vis. Science,(48): E-Abstract 6008.

Kaufman, P. L., L. Z. Bito and C. J. DeRousseau (1982). "The development of presbyopia in primates." Trans Ophthalmol Soc U K 102 Pt 3: 323-326.

Kelly, J. E., T. Mihashi and H. C. Howland (2004). "Compensation of corneal horizontal/vertical astigmatism, lateral coma, and spherical aberration by internal optics of the eye." J Vis 4(4): 262271.

Kepler, J. (1604). " Ad Vitellionem paralipomena.".

Kim, E., K. Ehrmann, S. Uhlhorn, D. Borja, E. Arrieta-Quintero and J. M. Parel (2011). "Semiautomated analysis of optical coherence tomography crystalline lens images under simulated accommodation." J Biomed Opt 16(5): 056003.

Kim, J. H., S. W. Kang, J. R. Kim and Y. S. Chang (2014). "Influence of image compression on the interpretation of spectral-domain optical coherence tomography in exudative age-related macular degeneration." Eye (Lond) 28(7): 825-831.

Kirschkamp, T., M. Dunne and J. C. Barry (2004). "Phakometric measurement of ocular surface radii of curvature, axial separations and alignment in relaxed and accommodated human eyes." Ophthalmic Physiol Opt 24(2): 65-73. 
Kling, S., Remon, L., Perez-Escudero, A., Merayo-Lloves, J., \& Marcos, S. (2009). "Biomechanical Response of Normal and Cross-linked Porcine Corneas." IOVS

ARVO E-Abstract: 5477.

Koh, S., N. Maeda, T. Kuroda, Y. Hori, H. Watanabe, T. Fujikado, Y. Tano, Y. Hirohara and T. Mihashi (2002). "Effect of tear film break-up on higher-order aberrations measured with wavefront sensor." Am J Ophthalmol 134(1): 115-117.

Kohnen, T. (1998). "Complications and complication management with foldable intraocular intraocular lenses." J Cataract Refract Surg 24(9): 1167-1168.

Konstantopoulos, A., P. Hossain and D. F. Anderson (2007). "Recent advances in ophthalmic anterior segment imaging: a new era for ophthalmic diagnosis?" Br J Ophthalmol 91(4): 551-557.

Koretz, J. E., S. A. Strenk, L. M. Strenk and J. L. Semmlow (2004). "Scheimpflug and highresolution magnetic resonance imaging of the anterior segment: a comparative study." J Opt Soc Am A Opt Image Sci Vis 21(3): 346-354.

Koretz, J. F., C. A. Cook and P. L. Kaufman (1997). "Accommodation and presbyopia in the human eye. Changes in the anterior segment and crystalline lens with focus." Invest Ophthalmol Vis Sci 38(3): 569-578.

Koretz, J. F., C. A. Cook and P. L. Kaufman (2001). "Aging of the human lens: changes in lens shape at zero-diopter accommodation." J Opt Soc Am A Opt Image Sci Vis 18(2): 265-272.

Koretz, J. F., G. H. Handelman and N. P. Brown (1984). "Analysis of human crystalline lens curvature as a function of accommodative state and age." Vision Res 24(10): 1141-1151.

Koretz, J. F., P. L. Kaufman, M. W. Neider and P. A. Goeckner (1989). "Accommodation and presbyopia in the human eye--aging of the anterior segment." Vision Res 29(12): 1685-1692.

Koszeghy, A., B. Pal, P. Pap, K. Pocsai, Z. Nagy, G. Szucs and Z. Rusznak (2009). "Purkinje-like cells of the rat cochlear nucleus: a combined functional and morphological study." Brain Res 1297: 57-69.

Kotecha, A., R. A. Russell, A. Sinapis, S. Pourjavan, D. Sinapis and D. F. Garway-Heath (2014). "Biomechanical parameters of the cornea measured with the Ocular Response Analyzer in normal eyes." BMC Ophthalmol 14: 11.

Kozaki, J., H. Tanihara, A. Yasuda and M. Nagata (1991). "Tilt and decentration of the implanted posterior chamber intraocular lens." J Cataract Refract Surg 17(5): 592-595.

Kretz, F. T., R. Khoramnia, M. S. Attia, M. J. Koss, K. Linz and G. U. Auffarth (2016). "Clinical Evaluation of Functional Vision of +1.5 Diopters near Addition, Aspheric, Rotational Asymmetric Multifocal Intraocular Lens." Korean J Ophthalmol 30(5): 382-389.

Kumar, D. A., A. Agarwal, S. Jacob and A. Agarwal (2013). "Glued trans-scleral intraocular lens exchange for anterior chamber lenses in complicated eyes: analysis of indications and results." Am J Ophthalmol 156(6): 1125-1133 e1122.

Kuroda, T., T. Fujikado, N. Maeda, T. Oshika, Y. Hirohara and T. Mihashi (2002). "Wavefront analysis of higher-order aberrations in patients with cataract." J Cataract Refract Surg 28(3): 438444.

Kymionis, G. D., A. E. Karavitaki, M. A. Grentzelos, D. A. Liakopoulos, K. I. Tsoulnaras and G. A. Kontadakis (2014). "Topography-based keratoconus progression after corneal collagen crosslinking." Cornea 33(4): 419-421.

$\mathbf{L}$ 
Lam, G. T., C. Jiang and C. S. Thummel (1997). "Coordination of larval and prepupal gene expression by the DHR3 orphan receptor during Drosophila metamorphosis." Development 124(9): 1757-1769.

Landau, I. M. and C. G. Laurell (1999). "Ultrasound biomicroscopy examination of intraocular lens haptic position after phacoemulsification with continuous curvilinear capsulorhexis and extracapsular cataract extraction with linear capsulotomy." Acta Ophthalmol Scand 77(4): 394-396.

Le Grand, Y. (1956). "Optique Physiologique " L'espace Visuel (Vol. 3)((1 ed. Vol. 3)): Paris: Éditions de la Revue d'Optique.

Lee, D. H., H. Y. Lee, K. H. Lee, K. H. Chung and C. K. Joo (2001). "Effect of a capsular tension ring on the shape of the capsular bag and opening and the intraocular lens." J Cataract Refract Surg 27(3): 452-456.

Lee, J. Y., Y. K. Lee, J. I. Moon and M. H. Park (2014). "Spectral domain optical coherence tomography cross-sectional image of optic nerve head during intraocular pressure elevation." Int J Ophthalmol 7(6): 1022-1029.

Lee, K. S., K. P. Thompson and J. P. Rolland (2010). "Broadband astigmatism-corrected CzernyTurner spectrometer." Opt Express 18(22): 23378-23384.

Leitgeb, R., C. Hitzenberger and A. Fercher (2003). "Performance of fourier domain vs. time domain optical coherence tomography." Opt Express 11(8): 889-894.

Letocha, C. E. and C. J. Pavlin (1999). "Follow-up of 3 patients with Ridley intraocular lens implantation." J Cataract Refract Surg 25(4): 587-591.

Lewis, R. (1983). "The Conquest of Presbyopia." Sydney, Austr.: R. \& M. Lewis.((p. 111)).

Lexer, F., C. K. Hitzenberger, A. F. Fercher and M. Kulhavy (1997). "Wavelength-tuning interferometry of intraocular distances." Appl Opt 36(25): 6548-6553.

Li, L., K. Wang, Y. Yan, X. Song and Z. Liu (2013). "Research on calculation of the IOL tilt and decentration based on surface fitting." Comput Math Methods Med 2013: 572530.

Li, Y., D. M. Meisler, M. Tang, A. T. Lu, V. Thakrar, B. J. Reiser and D. Huang (2008).

"Keratoconus diagnosis with optical coherence tomography pachymetry mapping." Ophthalmology 115(12): 2159-2166.

Lim, L., G. Gazzard, Y. H. Chan, A. Fong, A. Kotecha, E. L. Sim, D. Tan, L. Tong and S. M. Saw (2008). "Cornea biomechanical characteristics and their correlates with refractive error in Singaporean children." Invest Ophthalmol Vis Sci 49(9): 3852-3857.

Lin, Y. S., C. C. Chu, J. J. Lin, C. C. Chang, C. C. Wang, C. Y. Wang and P. H. Tsui (2014). "Optical coherence tomography: a new strategy to image planarian regeneration." Sci Rep 4: 6316.

Linebarger, E. J., D. R. Hardten, G. K. Shah and R. L. Lindstrom (1999). "Phacoemulsification and modern cataract surgery." Surv Ophthalmol 44(2): 123-147.

Liou, H. L. and N. A. Brennan (1997). "Anatomically accurate, finite model eye for optical modeling." J Opt Soc Am A Opt Image Sci Vis 14(8): 1684-1695.

Liu, C., A. Wong, P. Fieguth, K. Bizheva and H. Bie (2014). "Noise-compensated homotopic nonlocal regularized reconstruction for rapid retinal optical coherence tomography image acquisitions." BMC Med Imaging 14: 37.

Liu, Y. C., P. Chou, R. Wojciechowski, P. Y. Lin, C. J. Liu, S. J. Chen, J. H. Liu, W. M. Hsu and C. Y. Cheng (2011). "Power vector analysis of refractive, corneal, and internal astigmatism in an elderly Chinese population: the Shihpai Eye Study." Invest Ophthalmol Vis Sci 52(13): 9651-9657.

Llorente, L., S. Barbero, D. Cano, C. Dorronsoro and S. Marcos (2004). "Myopic versus hyperopic eyes: axial length, corneal shape and optical aberrations." J Vis 4(4): 288-298. 
Llorente, L., L. Diaz-Santana, D. Lara-Saucedo and S. Marcos (2003). "Aberrations of the human eye in visible and near infrared illumination." Optom Vis Sci 80(1): 26-35.

Long, D. A. and M. L. Monica (1996). "A prospective evaluation of corneal curvature changes with 3.0- to 3.5-mm corneal tunnel phacoemulsification." Ophthalmology 103(2): 226-232.

Loper, L. R. (1959). "The relationship between angle lambda and the residual astigmatism of the eye." Am J Optom Arch Am Acad Optom 36(7): 365-377.

Lowe, R. F. and B. A. Clark (1973). "Posterior corneal curvature. Correlations in normal eyes and in eyes involved with primary angle-closure glaucoma." Br J Ophthalmol 57(7): 464-470.

$\mathrm{Lu}, \mathrm{B}$. and Z. Shen (1999). "[Measurement and research of posterior chamber intraocular lens tilt and decentration in vivo]." Zhonghua Yan Ke Za Zhi 35(1): 40-42.

Lyle, W. A. and J. C. Jin (1992). "An analysis of intraocular lens exchange." Ophthalmic Surg 23(7): 453-458.

\section{M}

Maeda, N., T. Fujikado, T. Kuroda, T. Mihashi, Y. Hirohara, K. Nishida, H. Watanabe and Y. Tano (2002). "Wavefront aberrations measured with Hartmann-Shack sensor in patients with keratoconus." Ophthalmology 109(11): 1996-2003.

Maeda, P. Y. (2003). "Zernike Polynomials and Their Use in Describing the Wavefront Aberrations of the Human Eye." Psych 221/EE362 Applied Vision and Imaging Systems Course Project(Stanford University).

Mahajan, V. N. (1994). "Zernike circle polynomials and optical aberrations of systems with circular pupils." Appl Opt 33(34): 8121.

Majdi, J. A., H. Qian, Y. Li, R. J. Langsner, K. I. Shea, A. Agrawal, D. X. Hammer, J. P. Hanig and E. D. Cohen (2015). "The use of time-lapse optical coherence tomography to image the effects of microapplied toxins on the retina." Invest Ophthalmol Vis Sci 56(1): 587-597.

Malacara, D., Martin, J., Sánchez-Mondragón, J. J. (1990). "Wavefront fitting with discrete orthogonal polynomials in a unit radius circle. ." Optical Engineering, 29(6)(672-675).

Malyugin, B. E., A. A. Shpak and D. F. Pokrovskiy (2015). "Posterior chamber phakic intraocular lens sizing based on iris pigment layer measurements by anterior segment optical coherence tomography." J Cataract Refract Surg 41(8): 1616-1622.

Mamalis, N. (2002). "Complications of foldable intraocular lenses requiring explantation or secondary intervention--2001 survey update." J Cataract Refract Surg 28(12): 2193-2201.

Mandell, R. B. (1995). "Locating the corneal sighting center from videokeratography." J Refract Surg 11(4): 253-259.

Manns, F., V. Fernandez, S. Zipper, S. Sandadi, M. Hamaoui, A. Ho and J. M. Parel (2004). "Radius of curvature and asphericity of the anterior and posterior surface of human cadaver crystalline lenses." Exp Eye Res 78(1): 39-51.

Marcos, S., S. A. Burns, E. Moreno-Barriusop and R. Navarro (1999). "A new approach to the study of ocular chromatic aberrations." Vision Res 39(26): 4309-4323.

Marcos, S., S. A. Burns, P. M. Prieto, R. Navarro and B. Baraibar (2001). "Investigating sources of variability of monochromatic and transverse chromatic aberrations across eyes." Vision Res 41(28): 3861-3871.

Marcos, S., L. Diaz-Santana, L. Llorente and C. Dainty (2002). "Ocular aberrations with ray tracing and Shack-Hartmann wave-front sensors: does polarization play a role?" J Opt Soc Am A Opt Image Sci Vis 19(6): 1063-1072. 
Marcos, S., S. Ortiz, P. Perez-Merino, J. Birkenfeld, S. Duran and I. Jimenez-Alfaro (2014). "Threedimensional evaluation of accommodating intraocular lens shift and alignment in vivo." Ophthalmology 121(1): 45-55.

Marcos, S., P. Rosales, L. Llorente, S. Barbero and I. Jimenez-Alfaro (2008). "Balance of corneal horizontal coma by internal optics in eyes with intraocular artificial lenses: evidence of a passive mechanism." Vision Res 48(1): 70-79.

Marcos S., M.-E. E., Sun, M. , Perez-Merino P., Velasco-Ocana M. (2016). "3-D OCT ocular biometry." XXII Biennial Meeting of the International Society for Eye Research ISER - Abstract: A-817-0005-00262.

Marsack, J. D., L. N. Thibos and R. A. Applegate (2004). "Metrics of optical quality derived from wave aberrations predict visual performance." J Vis 4(4): 322-328.

Martinez-Enriquez, E., M. Sun, M. Velasco-Ocana, J. Birkenfeld, P. Perez-Merino and S. Marcos (2016). "Optical Coherence Tomography Based Estimates of Crystalline Lens Volume, Equatorial Diameter, and Plane Position." Invest Ophthalmol Vis Sci 57(9): OCT600-OCT610.

Martinez-Enriquez, E., M. Sun, M. Velasco-Ocana, J. Birkenfeld, P. Perez-Merino and S. Marcos (2016). "Optical Coherence Tomography Based Estimates of Crystalline Lens Volume, Equatorial Diameter, and Plane Position." Invest Ophthalmol Vis Sci 57(9): OCT600-610.

Martinez-Enriquez, E. P.-M., Pablo; Sun, Mengchan; Durán-Poveda, Sonia; Jiménez-Alfaro, Ignacio; Marcos, Susana (2017). "Full shape crystalline lens quantification from 3-D OCT images and its application to predict the post-operative IOL position." ARVO E-abstract 2686338.

Marussich, L., F. Manns, D. Nankivil, B. Maceo Heilman, Y. Yao, E. Arrieta-Quintero, A. Ho, R. Augusteyn and J. M. Parel (2015). "Measurement of Crystalline Lens Volume During Accommodation in a Lens Stretcher." Invest Ophthalmol Vis Sci 56(8): 4239-4248.

Mathur, A., D. A. Atchison and W. N. Charman (2009). "Myopia and peripheral ocular aberrations." J Vis 9(10): 15 11-12.

McGinty, S. J. and R. J. Truscott (2006). "Presbyopia: the first stage of nuclear cataract?" Ophthalmic Res 38(3): 137-148.

McLellan, J. S., S. Marcos and S. A. Burns (2001). "Age-related changes in monochromatic wave aberrations of the human eye." Invest Ophthalmol Vis Sci 42(6): 1390-1395.

McLeod, S. D. (2006). "Optical principles, biomechanics, and initial clinical performance of a dualoptic accommodating intraocular lens (an American Ophthalmological Society thesis)." Trans Am Ophthalmol Soc 104: 437-452.

McNabb, R. P., A. N. Kuo and J. A. Izatt (2013). "Quantitative single and multi-surface clinical corneal topography utilizing optical coherence tomography." Opt Lett 38(8): 1212-1214.

Meek, K. M. and C. Knupp (2015). "Corneal structure and transparency." Prog Retin Eye Res 49: $1-16$.

Meek, K. M. and R. H. Newton (1999). "Organization of collagen fibrils in the corneal stroma in relation to mechanical properties and surgical practice." J Refract Surg 15(6): 695-699.

MERINO, P. P. (2015). "ANTERIOR SEGMENT TOPOGRAPHY AND ABERRATIONS FOR CLINICAL APPLICATIONS." PhD thesis.

Michael J. Simpson, J. M. S., Son Trung Tran (2007). "Intraocular Lens with Asymmetric Haptics." Patentes US20080269881 A1(Candidatura): US 11/741,854.

Michael, R. and A. J. Bron (2011). "The ageing lens and cataract: a model of normal and pathological ageing." Philos Trans R Soc Lond B Biol Sci 366(1568): 1278-1292. 
Moffat, B. A., D. A. Atchison and J. M. Pope (2002). "Age-related changes in refractive index distribution and power of the human lens as measured by magnetic resonance micro-imaging in vitro." Vision Res 42(13): 1683-1693.

Molebny, V. V., Pallikaris, I. G., Naoumidis, L. P., Chyzh, I. H., Molebny, S. V., Sokurenko, V. M. (1997). "Retina Ray-Tracing technique for eye-refraction mapping." Proceedings of the SPIE, 2971(175-183).

Moreno-Barriuso, E., S. Marcos, R. Navarro and S. A. Burns (2001). "Comparing laser ray tracing, the spatially resolved refractometer, and the Hartmann-Shack sensor to measure the ocular wave aberration." Optom Vis Sci 78(3): 152-156.

Moreno-Barriuso, E., J. Merayo-Lloves, S. Marcos, R. Navarro, L. Llorente and S. Barbero (2001). "Ocular aberrations before and after myopic corneal refractive surgery: LASIK-induced changes measured with laser ray tracing." Invest Ophthalmol Vis Sci 42(6): 1396-1403.

Mutlu, F. M., A. Bayer, C. Erduman and M. Z. Bayraktar (2005). "Comparison of tilt and decentration between phacoemulsification and phacotrabeculectomy." Ophthalmologica 219(1): 26-29.

Mutti, D. O., K. Zadnik and A. J. Adams (1992). "A video technique for phakometry of the human crystalline lens." Invest Ophthalmol Vis Sci 33(5): 1771-1782.

\section{$\mathbf{N}$}

Napoletani, D. and T. D. Sauer (2008). "Reconstructing the topology of sparsely connected dynamical networks." Phys Rev E Stat Nonlin Soft Matter Phys 77(2 Pt 2): 026103.

Navarro, R. (2009). "The Optical Design of the Human Eye: a Critical Review." J Optom Vol. 2 (1): 3-18.

Navarro, R. and M. A. Losada (1997). "Aberrations and relative efficiency of light pencils in the living human eye." Optom Vis Sci 74(7): 540-547.

Navarro, R. and E. Moreno-Barriuso (1999). "Laser ray-tracing method for optical testing." Opt Lett 24(14): 951-953.

Navarro, R., E. Moreno and C. Dorronsoro (1998). "Monochromatic aberrations and point-spread functions of the human eye across the visual field." J Opt Soc Am A Opt Image Sci Vis 15(9): 25222529 .

Nishi, Y., N. Hirnschall, A. Crnej, V. Gangwani, J. Tabernero, P. Artal and O. Findl (2010). "Reproducibility of intraocular lens decentration and tilt measurement using a clinical Purkinje meter." J Cataract Refract Surg 36(9): 1529-1535.

Noorden., G. K. V. (1985). "Burian and Von Noorden's binocular vision and ocular motility." C. V. Mosby St. Louis(3rd edition).

Norrby, S. (2001). "Multicenter biometry study of 1 pair of eyes." J Cataract Refract Surg 27(10): 1656-1661.

Norrby, S. (2008). "Sources of error in intraocular lens power calculation." J Cataract Refract Surg 34(3): 368-376.

Norrby, S., E. Lydahl, G. Koranyi and M. Taube (2003). "Reduction of trend errors in power calculation by linear transformation of measured axial lengths." J Cataract Refract Surg 29(1): 100105. 
Obstbaum, S. A. and K. To (1989). "Posterior chamber intraocular lens dislocations and malpositions." Aust N Z J Ophthalmol 17(3): 265-271.

Ohara, K., S. Kato, S. Hori and S. Kitano (2006). "Tilt and decentration of the intraocular lens following combined vitrectomy and pars plana lensectomy." Acta Ophthalmol Scand 84(3): 388389.

Olsen, T. (1992). "Sources of error in intraocular lens power calculation." J Cataract Refract Surg 18(2): $125-129$.

Olsen, T. (2006). "Prediction of the effective postoperative (intraocular lens) anterior chamber depth." J Cataract Refract Surg 32(3): 419-424.

Olsen T (2004). "The Olsen formula. ." In: Shammas HJ, ed, Intraocular Lens Power Calculations. Thorofare, NJ, Slack, : 27-40.

Olsen, T., L. Corydon and H. Gimbel (1995). "Intraocular lens power calculation with an improved anterior chamber depth prediction algorithm." J Cataract Refract Surg 21(3): 313-319.

Olsen, T. and P. Hoffmann (2014). "C constant: new concept for ray tracing-assisted intraocular lens power calculation." J Cataract Refract Surg 40(5): 764-773.

Ortiz-Toquero, S., G. Rodriguez, V. de Juan and R. Martin (2014). "Repeatability of placido-based corneal topography in keratoconus." Optom Vis Sci 91(12): 1467-1473.

Ortiz, S., P. Perez-Merino, S. Duran, M. Velasco-Ocana, J. Birkenfeld, A. de Castro, I. JimenezAlfaro and S. Marcos (2013). "Full OCT anterior segment biometry: an application in cataract surgery." Biomed Opt Express 4(3): 387-396.

Ortiz, S., P. Perez-Merino, E. Gambra, A. de Castro and S. Marcos (2012). "In vivo human crystalline lens topography." Biomed Opt Express 3(10): 2471-2488.

Ortiz, S., D. Siedlecki, L. Remon and S. Marcos (2009). "Optical coherence tomography for quantitative surface topography." Appl Opt 48(35): 6708-6715.

Oshika, T., G. Sugita, K. Miyata, T. Tokunaga, T. Samejima, C. Okamoto and Y. Ishii (2007). "Influence of tilt and decentration of scleral-sutured intraocular lens on ocular higher-order wavefront aberration." Br J Ophthalmol 91(2): 185-188.

Ostrin, L., S. Kasthurirangan, D. Win-Hall and A. Glasser (2006). "Simultaneous measurements of refraction and A-scan biometry during accommodation in humans." Optom Vis Sci 83(9): 657-665.

\section{$\mathbf{P}$}

P. H. Kiely, G. S., and G. Carney (1982). "The mean shape of the human cornea." Opt. Acta 29(1027-1040).

Park, B., M. C. Pierce, B. Cense, S. H. Yun, M. Mujat, G. Tearney, B. Bouma and J. de Boer (2005). "Real-time fiber-based multi-functional spectral-domain optical coherence tomography at 1.3 microm." Opt Express 13(11): 3931-3944.

Park, C. Y., J. H. Oh and R. S. Chuck (2013). "Predicting ocular residual astigmatism using corneal and refractive parameters: a myopic eye study." Curr Eye Res 38(8): 851-861.

Pascolini, D., S. P. Mariotti, G. P. Pokharel, R. Pararajasegaram, D. Etya'ale, A. D. Negrel and S. Resnikoff (2004). "2002 global update of available data on visual impairment: a compilation of population-based prevalence studies." Ophthalmic Epidemiol 11(2): 67-115.

Patel, S., J. Marshall and F. W. Fitzke (1993). "Shape and radius of posterior corneal surface." Refract Corneal Surg 9(3): 173-181. 
Patel, S., J. Marshall and F. W. Fitzke, 3rd (1995). "Refractive index of the human corneal epithelium and stroma." J Refract Surg 11(2): 100-105.

Perez-Escudero, A., C. Dorronsoro and S. Marcos (2010). "Correlation between radius and asphericity in surfaces fitted by conics." J Opt Soc Am A Opt Image Sci Vis 27(7): 1541-1548.

Perez-Merino, P., J. Birkenfeld, C. Dorronsoro, S. Ortiz, S. Duran, I. Jimenez-Alfaro and S. Marcos (2014). "Aberrometry in patients implanted with accommodative intraocular lenses." Ophthalmol 157(5): 1077-1089.

Perez-Merino, P., C. Dorronsoro, L. Llorente, S. Duran, I. Jimenez-Alfaro and S. Marcos (2013). "In vivo chromatic aberration in eyes implanted with intraocular lenses." Invest Ophthalmol Vis Sci 54(4): 2654-2661.

Perez-Merino, P., S. Ortiz, N. Alejandre, A. de Castro, I. Jimenez-Alfaro and S. Marcos (2014). "Ocular and optical coherence tomography-based corneal aberrometry in keratoconic eyes treated by intracorneal ring segments." Am J Ophthalmol 157(1): 116-127 e111.

Petermeier, K., F. Gekeler, A. Messias, M. S. Spitzer, W. Haigis and P. Szurman (2009). "Intraocular lens power calculation and optimized constants for highly myopic eyes." J Cataract Refract Surg 35(9): 1575-1581.

Phillips, P., J. Perez-Emmanuelli, H. D. Rosskothen and C. J. Koester (1988). "Measurement of intraocular lens decentration and tilt in vivo." J Cataract Refract Surg 14(2): 129-135.

Pierscionek, B. K. (1990). "Presbyopia - effect of refractive index." Clin. Exp. Optom. 73((1)): 2330.

Pierscionek, B. K. and D. Y. Chan (1989). "Refractive index gradient of human lenses." Optom Vis Sci 66(12): 822-829.

Pomerantzeff, O., M. Pankratov, G. J. Wang and P. Dufault (1984). "Wide-angle optical model of the eye." Am J Optom Physiol Opt 61(3): 166-176.

Preussner, P. R., J. Wahl, H. Lahdo, B. Dick and O. Findl (2002). "Ray tracing for intraocular lens calculation." J Cataract Refract Surg 28(8): 1412-1419.

\section{$\mathbf{R}$}

Rao, H. L., A. U. Kumar, A. Kumar, S. Chary, S. Senthil, P. K. Vaddavalli and C. S. Garudadri (2011). "Evaluation of central corneal thickness measurement with RTVue spectral domain optical coherence tomography in normal subjects." Cornea 30(2): 121-126.

Rao, S. K. and P. Padmanabhan (1998). "Posterior keratoconus. An expanded classification scheme based on corneal topography." Ophthalmology 105(7): 1206-1212.

Resnikoff, S., D. Pascolini, D. Etya'ale, I. Kocur, R. Pararajasegaram, G. P. Pokharel and S. P. Mariotti (2004). "Global data on visual impairment in the year 2002." Bull World Health Organ 82(11): 844-851.

Resnikoff, S., D. Pascolini, S. P. Mariotti and G. P. Pokharel (2008). "Global magnitude of visual impairment caused by uncorrected refractive errors in 2004." Bull World Health Organ 86(1): 6370 .

Retzlaff, J. A., D. R. Sanders and M. C. Kraff (1990). "Development of the SRK/T intraocular lens implant power calculation formula." J Cataract Refract Surg 16(3): 333-340.

Richards, D. W., S. R. Russell and D. R. Anderson (1988). "A method for improved biometry of the anterior chamber with a Scheimpflug technique." Invest Ophthalmol Vis Sci 29(12): 1826-1835. 
Rizzo, S., C. Basso, G. Buja, M. Valente and G. Thiene (2014). "Multifocal Purkinje-like hamartoma and junctional ectopic tachycardia with a rapidly fatal outcome in a newborn." Heart Rhythm 11(7): 1264-1266.

Roberts, C. (2000). "The cornea is not a piece of plastic." J Refract Surg 16(4): 407-413.

Rohart, C. and D. Gatinel (2009). "Influence of a capsular tension ring on ocular aberrations after cataract surgery: a comparative study." J Refract Surg 25(1 Suppl): S116-121.

Roorda, A. and A. Glasser (2004). "Wave aberrations of the isolated crystalline lens." J Vis 4(4): 250-261.

Rosales, P., A. De Castro, I. Jimenez-Alfaro and S. Marcos (2010). "Intraocular lens alignment from purkinje and Scheimpflug imaging." Clin Exp Optom 93(6): 400-408.

Rosales, P., M. Dubbelman, S. Marcos and R. van der Heijde (2006). "Crystalline lens radii of curvature from Purkinje and Scheimpflug imaging." J Vis 6(10): 1057-1067.

Rosales, P. and S. Marcos (2006). "Phakometry and lens tilt and decentration using a customdeveloped Purkinje imaging apparatus: validation and measurements." J Opt Soc Am A Opt Image Sci Vis 23(3): 509-520.

Rosales, P. and S. Marcos (2007). "Customized computer models of eyes with intraocular lenses." Opt Express 15(5): 2204-2218.

Rosales, P. and S. Marcos (2009). "Pentacam Scheimpflug quantitative imaging of the crystalline lens and intraocular lens." J Refract Surg 25(5): 421-428.

Rosales, P., M. Wendt, S. Marcos and A. Glasser (2008). "Changes in crystalline lens radii of curvature and lens tilt and decentration during dynamic accommodation in rhesus monkeys." J Vis 8(1): 18 11-12.

Rosen, A. M., D. B. Denham, V. Fernandez, D. Borja, A. Ho, F. Manns, J. M. Parel and R. C. Augusteyn (2006). "In vitro dimensions and curvatures of human lenses." Vision Res 46(6-7): 10021009 .

Rossi, L., R. Piffer, E. Turolla, B. Frigerio, P. Coumel and T. N. James (1985). "Multifocal Purkinjelike tumor of the heart. Occurrence with other anatomic abnormalities in the atrioventricular junction of an infant with junctional tachycardia, Lown-Ganong-Levine syndrome, and sudden death." Chest 87(3): 340-345.

\section{$\mathbf{S}$}

S Marcos, M. L.-Q., R Niembro and L N Savushkin (2005). "The pseudospin symmetry in atomic nuclei." Journal of Physics G: Nuclear and Particle Physics 31: 10.

Salomao, M. Q., A. Esposito and W. J. Dupps, Jr. (2009). "Advances in anterior segment imaging and analysis." Curr Opin Ophthalmol 20(4): 324-332.

Sanders, D. R. and M. L. Sanders (2007). "Visual performance results after Tetraflex accommodating intraocular lens implantation." Ophthalmology 114(9): 1679-1684.

Sauer, T. D. (2002). "Shadowing breakdown and large errors in dynamical simulations of physical systems." Phys Rev E Stat Nonlin Soft Matter Phys 65(3 Pt 2A): 036220.

Sauer, T. D. (2004). "Reconstruction of shared nonlinear dynamics in a network." Phys Rev Lett 93(19): 198701.

Sauer, T. D. and S. J. Schiff (2012). "Rainfall drives hydrocephalus in East Africa." Phys Rev E Stat Nonlin Soft Matter Phys 10(3): 161-167. 
Sawtell, N. B., A. Williams and C. C. Bell (2007). "Central control of dendritic spikes shapes the responses of Purkinje-like cells through spike timing-dependent synaptic plasticity." J Neurosci 27(7): 1552-1565.

Scherini, E. and G. Bernocchi (1989). "Ectopic Purkinje-like cells are GABAergic: immunohistochemistry with an immune serum against glutamic acid decarboxylase." Cell Tissue Res 258(2): 437-439.

Schwiegerling, J., J. E. Greivenkamp and J. M. Miller (1995). "Representation of videokeratoscopic height data with Zernike polynomials." J Opt Soc Am A Opt Image Sci Vis 12(10): 2105-2113.

Shack, R., Platt, B. (1971). "Production and use of a lenticular Hartmann screen. ." J. Opt. Soc. Am. 61(656).

Sheehan, M. T. (2012). "Eye modelling for personalised intraocular lens design." $\mathrm{PhD}$ thesis Applied Optics Group, School of Physics, Science Faculty,(National University of Ireland, Galway).

Sheppard, A. L., C. J. Evans, K. D. Singh, J. S. Wolffsohn, M. C. Dunne and L. N. Davies (2011). "Three-dimensional magnetic resonance imaging of the phakic crystalline lens during accommodation." Invest Ophthalmol Vis Sci 52(6): 3689-3697.

Sicam, V. A., J. Coppens, T. J. van den Berg and R. G. van der Heijde (2004). "Corneal surface reconstruction algorithm that uses Zernike polynomial representation." J Opt Soc Am A Opt Image Sci Vis 21(7): 1300-1306.

Siedlecki, D., A. de Castro, E. Gambra, S. Ortiz, D. Borja, S. Uhlhorn, F. Manns, S. Marcos and J. M. Parel (2012). "Distortion correction of OCT images of the crystalline lens: gradient index approach." Optom Vis Sci 89(5): E709-718.

Simonet, P. and M. C. Campbell (1990). "The optical transverse chromatic aberration on the fovea of the human eye." Vision Res 30(2): 187-206.

Sinha, A. M., M. Schmidt, H. Marschang, K. Gutleben, G. Ritscher, J. Brachmann and N. F. Marrouche (2009). "Role of left ventricular scar and Purkinje-like potentials during mapping and ablation of ventricular fibrillation in dilated cardiomyopathy." Pacing Clin Electrophysiol 32(3): 286-290.

Sivak, J. G. and R. O. Kreuzer (1983). "Spherical aberration of the crystalline lens." Vision Res 23(1): 59-70.

Sivak, J. G. and T. Mandelman (1982). "Chromatic dispersion of the ocular media." Vision Res 22(8): 997-1003.

Smirnov, M. S. (1961). "Measurement of the wave aberration of the human eye." Biofizika 6: 776795 .

Smolek, M. K. and S. D. Klyce (2000). "Is keratoconus a true ectasia? An evaluation of corneal surface area." Arch Ophthalmol 118(9): 1179-1186.

Sorsby, A., B. Benjamin, J. B. Davey, M. Sheridan and J. M. Tanner (1957). "Emmetropia and its aberrations; a study in the correlation of the optical components of the eye." Spec Rep Ser Med Res Counc (G B) 11(293): 1-69.

Spatz, W. B. (1997). "Differences between guinea pig and rat in the dorsal cochlear nucleus: expression of calcium-binding proteins by cartwheel and Purkinje-like cells." Hear Res 107(1-2): 136-146.

Spatz, W. B. (2003). "Purkinje-like cells in the cochlear nucleus of the Common Tree Shrew (Tupaia glis) identified by calbindin immunohistochemistry." Brain Res 983(1-2): 230-232.

Spierer, A., Shalev, B. (2003). "Presbyopia among normal individuals." Graefes Arch Clin Exp Ophthalmol. 241(101-105.). 
Strenk, S., Strenk, L., Koretz, J.F. (2005). "The mechanism of presbyopia. ." Prog Retin Eye Res. 24(379-393).

Strenk, S. A., J. L. Semmlow, L. M. Strenk, P. Munoz, J. Gronlund-Jacob and J. K. DeMarco (1999). "Age-related changes in human ciliary muscle and lens: a magnetic resonance imaging study." Invest Ophthalmol Vis Sci 40(6): 1162-1169.

Strenk, S. A., L. M. Strenk and J. L. Semmlow (2000). "High resolution MRI study of circumlental space in the aging eye." J Refract Surg 16(5): S659-660.

Strenk, S. A., L. M. Strenk, J. L. Semmlow and J. K. DeMarco (2004). "Magnetic resonance imaging study of the effects of age and accommodation on the human lens cross-sectional area." Invest Ophthalmol Vis Sci 45(2): 539-545.

Strenn, K., R. Menapace and C. Vass (1997). "Capsular bag shrinkage after implantation of an openloop silicone lens and a poly(methyl methacrylate) capsule tension ring." J Cataract Refract Surg 23(10): 1543-1547.

Su, R., P. Ekberg, M. Leitner and L. Mattsson (2014). "Accurate and automated image segmentation of 3D optical coherence tomography data suffering from low signal-to-noise levels." J Opt Soc Am A Opt Image Sci Vis 31(12): 2551-2560.

Sun, M., J. Birkenfeld, A. de Castro, S. Ortiz and S. Marcos (2014). "OCT 3-D surface topography of isolated human crystalline lenses." Biomed Opt Express 5(10): 3547-3561.

Sun, M., A. de Castro, S. Ortiz, P. Perez-Merino, J. Birkenfeld and S. Marcos (2014). "Intraocular lens alignment from an en-face optical coherence tomography image Purkinje-like method." Optical Engineering 53(6): 061704-061704.

Sun, M., P. Pérez-Merino, E. Martinez-Enriquez, M. Velasco-Ocana and S. Marcos (2016). "Full 3D OCT-based pseudophakic custom computer eye model." Biomedical Optics Express 7(3): 10741088.

Sun, R. (2007). "Functions of the capsular tension ring." J Cataract Refract Surg 33(1): 4.

Susana Marcos, E. M.-E., Pablo Perez-Merino, Miriam Velasco-Ocana, Mengchan Sun (2016). "Unraveling eye crystalline lens optics, structure and function." Optical Society of America Latin America Optics and Photonics Conference(LTh2C. 1).

Susana Marcos , S. A. B. (2000). "On the symmetry between eyes of wavefront aberration and cone directionality." Vision Research 40(18): 2437-2447.

Svechnikov, M. V., N. I. Chkhalo, M. N. Toropov and N. N. Salashchenko (2015). "Resolving capacity of the circular Zernike polynomials." Opt Express 23(11): 14677-14694.

Swanson, E. A., J. A. Izatt, M. R. Hee, D. Huang, C. P. Lin, J. S. Schuman, C. A. Puliafito and J. G. Fujimoto (1993). "In vivo retinal imaging by optical coherence tomography." Opt Lett 18(21): 1864-1866.

Szigeti, A., E. Tatrai, A. Szamosi, P. Vargha, Z. Z. Nagy, J. Nemeth, D. C. DeBuc and G. M. Somfai (2014). "A morphological study of retinal changes in unilateral amblyopia using optical coherence tomography image segmentation." PLoS One 9(2): e88363.

\section{$\mathbf{T}$}

Tabernero, J., P. Piers, A. Benito, M. Redondo and P. Artal (2006). "Predicting the optical performance of eyes implanted with IOLs to correct spherical aberration." Invest Ophthalmol Vis Sci 47(10): 4651-4658.

Taketani, F., T. Matuura, E. Yukawa and Y. Hara (2004). "Influence of intraocular lens tilt and decentration on wavefront aberrations." J Cataract Refract Surg 30(10): 2158-2162. 
Tappin, M. J. and D. F. Larkin (2000). "Factors leading to lens implant decentration and exchange." Eye (Lond) 14 Pt 5: 773-776.

Tearney, G. J., M. E. Brezinski, B. E. Bouma, S. A. Boppart, C. Pitris, J. F. Southern and J. G. Fujimoto (1997). "In vivo endoscopic optical biopsy with optical coherence tomography." Science 276(5321): 2037-2039.

Thibos, L. N., R. A. Applegate, J. T. Schwiegerling, R. Webb, V. S. T. M. V. science and a. its (2002). "Standards for reporting the optical aberrations of eyes." J Refract Surg 18(5): S652-660.

Thibos, L. N., X. Hong, A. Bradley and X. Cheng (2002). "Statistical variation of aberration structure and image quality in a normal population of healthy eyes." J Opt Soc Am A Opt Image Sci Vis 19(12): 2329-2348.

Thibos, L. N. and D. Horner (2001). "Power vector analysis of the optical outcome of refractive surgery." J Cataract Refract Surg 27(1): 80-85.

Truscott, R. J. and X. Zhu (2010). "Presbyopia and cataract: a question of heat and time." Prog Retin Eye Res 29(6): 487-499.

Tscherning, M. (1894). " Die monochromatischen Aberrationen des menschlichen Auges." $\underline{Z}$ Psychol Physiola Sinne 6: 456-471.

\section{$\mathbf{U}$}

Uhlhorn, S. R., Borja, D., Manns, F., and Parel, J. M. (2008). "Refractive index measurement of the isolated crystalline lens using optical coherence tomography." Vision Res 48(27)(2732-2738).

Urs, R., F. Manns, A. Ho, D. Borja, A. Amelinckx, J. Smith, R. Jain, R. Augusteyn and J. M. Parel (2009). "Shape of the isolated ex-vivo human crystalline lens." Vision Res 49(1): 74-83.

\section{V}

Venter, J. A., M. Pelouskova, B. M. Collins, S. C. Schallhorn and S. J. Hannan (2013). "Visual outcomes and patient satisfaction in 9366 eyes using a refractive segmented multifocal intraocular lens." J Cataract Refract Surg 39(10): 1477-1484.

Vinas, M., C. Dorronsoro, D. Cortes, D. Pascual and S. Marcos (2015). "Longitudinal chromatic aberration of the human eye in the visible and near infrared from wavefront sensing, double-pass and psychophysics." Biomed Opt Express 6(3): 948-962.

Vitale, S., L. Ellwein, M. F. Cotch, F. L. Ferris, 3rd and R. Sperduto (2008). "Prevalence of refractive error in the United States, 1999-2004." Arch Ophthalmol 126(8): 1111-1119.

Von Helmholtz, H. (1909). "Helmholtz's Treatise on Physiological Optics." New York:Optical Society of America.

\section{W}

Wang, Z., H. Jia, J. Tian, T. Soeda, R. Vergallo, Y. Minami, H. Lee, A. Aguirre, J. G. Fujimoto and I. K. Jang (2014). "Computer-aided image analysis algorithm to enhance in vivo diagnosis of plaque erosion by intravascular optical coherence tomography." Circ Cardiovasc Imaging 7(5): 805-810.

Weeber, H. A. and R. G. van der Heijde (2007). "On the relationship between lens stiffness and accommodative amplitude." Exp Eye Res 85(5): 602-607. 
Weisstein, E. W. "Spherical Bessel Function of the Second Kind." MathWorld--A WolframWebResource. $\quad$ http://mathworld.wolfram.com/SphericalBesselFunctionof theSecondKind.html.

Whalen, A. J., S. N. Brennan, T. D. Sauer and S. J. Schiff (2012). "Observability of Neuronal Network Motifs." Proc Conf Inf Sci Syst 2012.

Wojtkowski, M. (2010). "High-speed optical coherence tomography: basics and applications." Appl Opt 49(16): D30-61.

Wojtkowski, M., T. Bajraszewski, P. Targowski and A. Kowalczyk (2003). "Real-time in vivo imaging by high-speed spectral optical coherence tomography." Opt Lett 28(19): 1745-1747.

Wojtkowski, M., A. Kowalczyk, R. Leitgeb and A. F. Fercher (2002). "Full range complex spectral optical coherence tomography technique in eye imaging." Opt Lett 27(16): 1415-1417.

Wolffsohn, J. S. and L. N. Davies (2007). "Advances in anterior segment imaging." Curr Opin Ophthalmol 18(1): 32-38.

Wu, M., H. Li and W. Cheng (1998). "Determination of intraocular lens tilt and decentration using simple and rapid method." Yan Ke Xue Bao 14(1): 13-16, 26.

\section{$\mathbf{X}$}

Xing, X. J., X. Tang, H. Song and W. W. Li (2010). "[Comparison of tilt and decentration of four different kinds of aspheric intraocular lenses implantation]." Zhonghua Yan Ke Za Zhi 46(4): 332336.

Xu, D., Y. Huang and J. U. Kang (2014). "Real-time dispersion-compensated image reconstruction for compressive sensing spectral domain optical coherence tomography." J Opt Soc Am A Opt Image Sci Vis 31(9): 2064-2069.

\section{$\mathbf{Y}$}

Yu, A., Q. Wang, S. Zhu, A. Xue, Y. Su and R. Pan (2015). "[Effects of posterior chamber phakic intraocular lens on axial length measurements]." Zhonghua Yan Ke Za Zhi 51(3): 206-209.

Yu, F., P. Chang, J. Li, Y. Zhou and Y. Zhao (2015). "[Comparative study of the tilt, decentration and higher-order aberrations (HOA) of single-piece and 3-piece tecnis aspheric intraocular lenses]." Zhonghua Yan Ke Za Zhi 51(4): 270-275.

Yun, S., G. Tearney, J. de Boer, N. Iftimia and B. Bouma (2003). "High-speed optical frequencydomain imaging." Opt Express 11(22): 2953-2963.

\section{$\mathbf{Z}$}

Zadnik, K., D. O. Mutti and A. J. Adams (1992). "The repeatability of measurement of the ocular components." Invest Ophthalmol Vis Sci 33(7): 2325-2333.

Zhao, M., A. N. Kuo and J. A. Izatt (2010). "3D refraction correction and extraction of clinical parameters from spectral domain optical coherence tomography of the cornea." Opt Express 18(9): 8923-8936.

Zuberbuhler, B. and A. J. Morrell (2007). "Errata in printed Hoffer Q formula." J Cataract Refract Surg 33(1): 2; author reply 2-3. 


\section{ACKNOWLEDGMENTS}

Being a $\mathrm{PhD}$ is both exciting and educational, but also quite demanding and fraught with thousands of failures and reverses, and I could never have managed on my own. I therefore wish to express my gratitude to each and every one, who helped and encouraged me in one way or another during these 5 years of PhD study. It has been an incredible experience and a wonderful memory.

First and foremost, I want to send many warm thanks to is my supervisor Prof. Susana Marcos, for offering the great opportunity to work in Visual Optics and Biophotonics (VioBio) Lab, in particular, her strong involvement in this $\mathrm{PhD}$ research, her invaluable guidance during those five years, and her impressive dedication to science. I am so proud to be a member of VioBio Lab, work with all lovely lab members and have the privilege of meeting many people: physicists, chemists, optometrists, ophthalmologists, biologists, electrical and mechanical engineers!

My acknowledgements to OpAL-Marie Curie ITN funding that supported my first three years of stay in Madrid, and the travels to the annual meetings and conferences. I am indebted to Prof. Frank Schaeffel at the Laboratory of University Hospital Tübingen (Tübingen, Germany), Prof. Ioannis Pallikaris and Prof. Harilaos Ginis atthe Laboratory of Optics and Imaging in the premises of the Department of Medicine (Crete, Greek), Prof. Linda Lundström and Prof. Peter Unsbo at the Laboratory of Applied Physics/ Biomedical and X-Ray Physics in AlboNova University center (Stockholm, Sweden), and Prof. Pablo Perez Artal at the University of Murcia in the Research Center for Optics and Nanophysics (Murcia, Spain), for valuable training courses, scientific discussions and collaborations. Thanks to Instituto de Óptica (IO), CSIC, especially to D. Eloy Belda San Mateo and D. José Luis Revuelta Gonzalez, for providing a productive environment with great assistance in these five years, Fundación Jiménez Díaz Hospital (FJD), specially to Dr. Sonia Durán for providing volunteer subjects participating in the measurements and supports from IOBA-Universidad de Valladolid and Univerisity of Miami/ Bascom Palmer Eye Institute, specially Prof. José Miguel Maldonado López and Prof. Fabrice Manns are also acknowledged.

My heartfelt thanks to Alberto de Castro, who is like a co-supervisor in both research work and otherwise, with plenty of patience and guidance, since the first day I worked in VioBio. Of course, he is the first reviewer of this thesis as well. Although he left the lab one year after I joined, we still keep in touch by email to share informal discussions of projects, and to relieve helplessness and stress when I met project bottlenecks. I am also thankful to Lucie Sawides. You guys are such a lovely couple. I felt that you are always sharing my daily life in email, and especially thanks for your wedding wish record in Chinese. How touching it was!

A special mention about our current OCT team members: Pablo Perez, Eduardo Martinez Enriquez and Mirian Velasco. Pablo is more than a work partner, he is like a family member with an infectious optimistic attitude in the face of any problem, with unquantifiable 
support and suggestions of solutions, no matter whether in work or in life (such as helping me review the manuscripts, rehearsing the presentations, eyewitness my wedding ceremony in Shanghai and let me eyewitness your wedding as well, etc.) Eduardede, thanks for helping me process OCT images, which is the first and key step in the whole thesis research. Although you always have crazy and incomprehensible whimsy, the lab is full of laughing, relaxation and pleasure owning to you. Mirian Velasco, owning to my poor Spanish, we did not communicate a lot at the beginning except working, but our relationship grew closer and closer after the invitations and attendances of your wedding and "after work workshop". Judith Birkenfeld and Sergio Ortiz Egea are ex OCT team members, both of you had brilliant careers after your left. I learned rigor, as a famous German character, from Judith, who offered invaluable helps in those five years and thanks to Sergio, who is the largest contributor in the OCT lab for the OCT image processing. I could not finish this thesis research without your contribution.

Many warm thanks go to James A Germann. Although we met about one year ago, I feel like we are very old and close friends. I am shy and dumb as an oyster, but your enthusiasm gives me great inspiration! I very much enjoyed sharing our thoughts, and learning new things from you every day! James A Germann did all the English spelling and grammar correction of this thesis using his busy time under high pressure projects. I do not know how to express my appreciation, but I know that I could not graduate without you! He is also one of brave members in the China adventure trip for my wedding, with Maria Viñas Peña, Rocío Gutiérrez Contreras, and Clara Benedi Carcia. Maria is the best example of working hard in research in Viobio, and I am so proud of you. I learned organizational skills, eloquence, serious attitude from the IOSA student chapter under her, Andres de la Hoz Duran and Mario's sponsorship and feel honor to be invited her wedding as well. Thanks Rocío and Clara for invitations of family lunches, which cured my home-sickness a lot. Clara even teaches me how to swim with her after work, at personal cost and with great patience. What's more, we have had a lot of travel memories together (Granada trip with Rocio and James, Zaragoza trip with Clara, Rocio, and James, ARVO trips with Maria, IOSA with you all, etc.). I have enjoyed every moment of your company during those trips. Thanks to you four, for taking our friendship outside of CSIC.

Another unforgettable person I need to say thanks to is Aiswaryah Radhakrishnan, who is another OpAL fellowship in the Lab. We shared almost all our PhD lives together with each annual meetings, conferences, training, scientific / non-scientific trips, and of course, Office 301 as well. Thanks so much for thoughtful comments and inquisitive suggestions of project results. You could always be counted on whenever I need Spanish translations and help for any trivial and daily thing. I am also much grateful to Enrique Bustos, Nohelia morales, Aida Llera, and Carmen Fernandezfor guiding me through all the paperwork (ARVO registrations, homologations, NIE extensions or contract extensions, etc.)

Thanks to Nandor Bekesi, Vyas Akondi, Enrique Gambra, Carlos Dorronsoro, Alvaro Sanchez-Lozano, Daniel Pascual, Nicolas Alejandre Alba, Sergio Barbero, Javier Portilla, etc, for your helpful suggestions, discussions, assistances and humor through all these years in so many things. It is being an unbelievable asset for me. 
Thanks to my family (孙国平, 张菊萍, 郑俭, and 沈璐琳), my husband (郑哲). You are one of the central pillars of my life and sustain who I am and who I want to be, supporting me without any grumble and enduring long separation and my friends (王莉, 杨起帆, 柏 园雅，Alejandro Garcia Ruiz，力祎，韩夏，张松丽，江小松，郭霄，and 陈末亚） and who I have not mentioned here but did any form of help to me. All of you are my powerful spiritual pillar to support me.

This thesis research was funded by the following grants, in which Prof. Susana Marcos was the Principal Investigator: This study has been funded by Spanish Government Grant FIS2011-25637 and European Research Council ERC-2011 AdG-294099 "Bio-inspired optical correction of presbyopia" to $S$. Marcos, and Marie Curie Actions-Networks of Initial Training (ITN) FP7-PEOPLE-2010-ITN 264605 (OpAL-Optical and adaptational limits of vision). We acknowledge the collaborative agreement "Unidad Asociada Tecnología e Innovación sanitaria en Oftalmología” (IIS Fundación Jiménez Díaz / IO-CSIC).

最后, 要把我最最珍贵的感谢送给我最亲爱的父母。在过去的 20 多年里, 无论我在 上海, 荷兰, 瑞典, 还是西班牙, 感谢你们都无微不至地支持, 照顾和鼓励。在 过去 8 年多的 3,000 多个日日夜夜, 我一直在遥远的欧洲思念着你们。

Thank you all from heart!!

Muchas sinceros agradecimientos a todos!!!

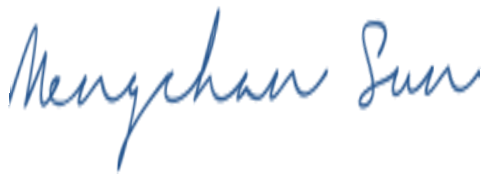

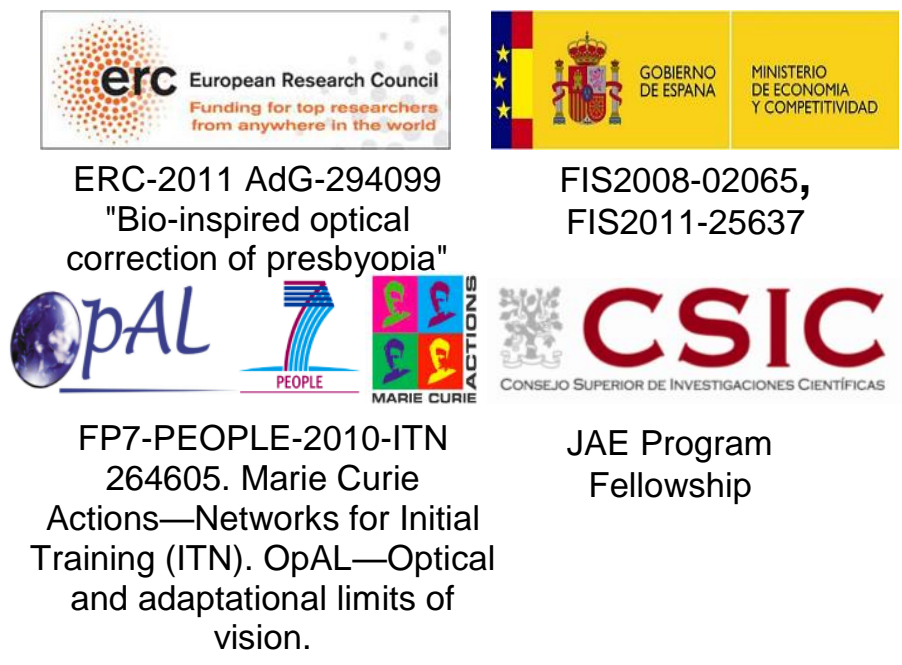



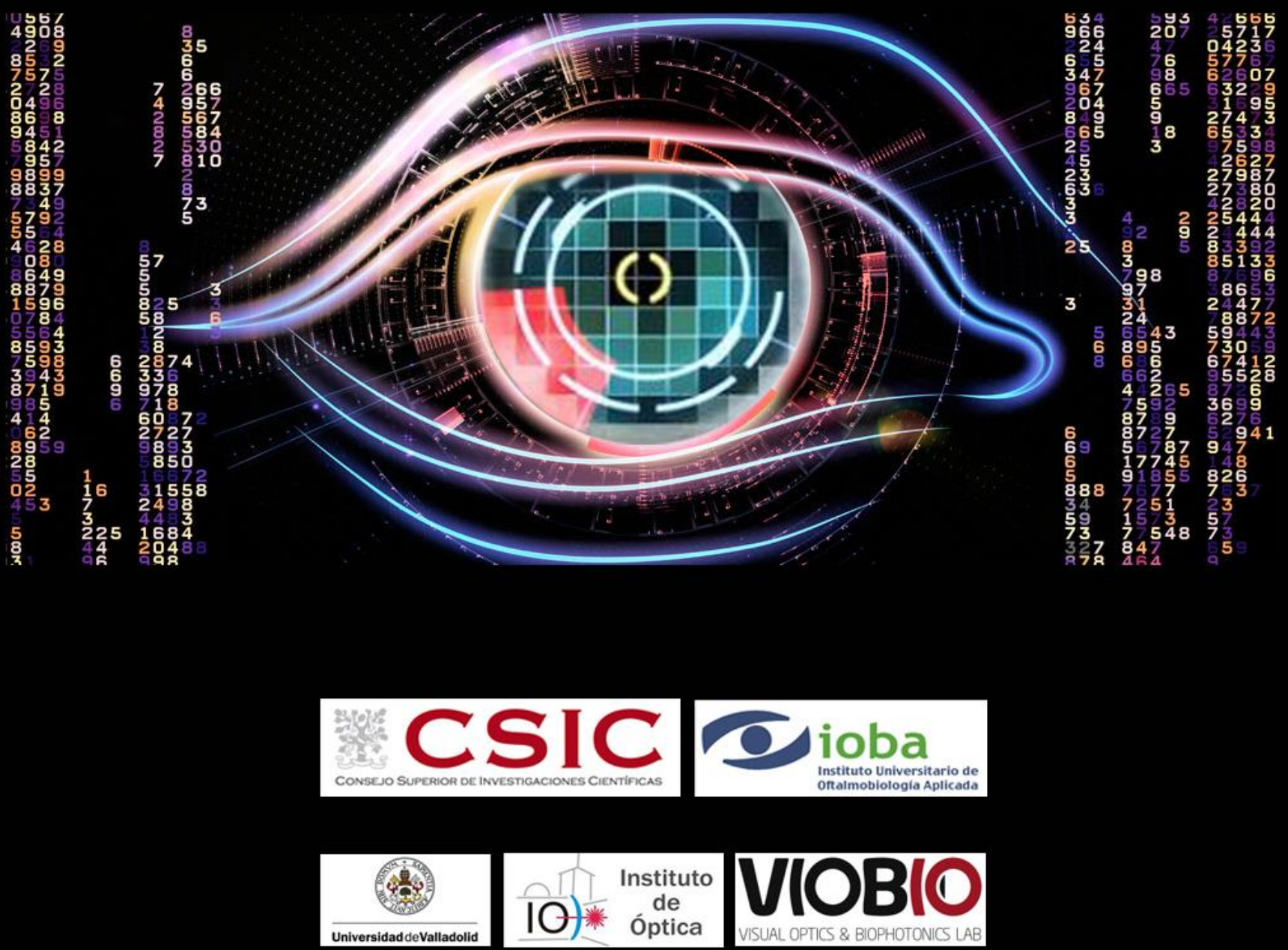